University of Louisville

ThinkIR: The University of Louisville's Institutional Repository

Electronic Theses and Dissertations

$5-2017$

\title{
Social dynamics, network structure, and information diffusion in fish shoals.
}

Matthew Jerome Hasenjager

University of Louisville

Follow this and additional works at: https://ir.library.louisville.edu/etd

Part of the Biology Commons

\section{Recommended Citation}

Hasenjager, Matthew Jerome, "Social dynamics, network structure, and information diffusion in fish shoals." (2017). Electronic Theses and Dissertations. Paper 2628.

https://doi.org/10.18297/etd/2628

This Doctoral Dissertation is brought to you for free and open access by ThinkIR: The University of Louisville's Institutional Repository. It has been accepted for inclusion in Electronic Theses and Dissertations by an authorized administrator of ThinkIR: The University of Louisville's Institutional Repository. This title appears here courtesy of the author, who has retained all other copyrights. For more information, please contact thinkir@louisville.edu. 
SOCIAL DYNAMICS, NETWORK STRUCTURE, AND INFORMATION DIFFUSION IN FISH SHOALS

\author{
By \\ Matthew Jerome Hasenjager \\ B.S., Michigan State University, 2009 \\ M.S., Michigan State University, 2011

\begin{abstract}
A Dissertation
Submitted to the Faculty of the

College of Arts and Sciences of the University of Louisville

in Partial Fulfillment of the Requirements

for the Degree of
\end{abstract}
Doctor of Philosophy
in Biology
Department of Biology
University of Louisville
Louisville, Kentucky

May 2017 
Copyright 2017 by Matthew Jerome Hasenjager

All rights reserved 

SOCIAL DYNAMICS, NETWORK STRUCTURE, AND INFORMATION DIFFUSION IN FISH SHOALS

\section{By}

Matthew Jerome Hasenjager

B.S., Michigan State University, 2009

M.S., Michigan State University, 2011

A Dissertation Approved on

April 26, 2017

by the following Dissertation Committee:

Dissertation Director

Dr. Lee A. Dugatkin

Dr. Cynthia Corbitt

Dr. Perri Eason

Dr. Paul Ewald

Dr. Thomas Valone 


\title{
DEDICATION
}

\author{
To my parents
}

Jerome and Margaret Hasenjager

for always ensuring my library was well-stocked, and for fostering my enduring love of the natural world. 


\section{ACKNOWLEDGEMENTS}

First and foremost, I would like to thank my advisor, Dr. Lee Dugatkin, for his guidance and support, and for the amazing opportunities he has provided me over my doctoral career. I would like to also thank the rest of my dissertation committee: Dr. Cynthia Corbitt, Dr. Perri Eason, Dr. Paul Ewald, and Dr. Thomas Valone, for all of their constructive feedback. Thank you, as well, to Dr. Stefan Krause for assistance with implementing the Markov chain fission-fusion models used in this dissertation and to Dr. William Hoppitt for answering my innumerable questions regarding network-based diffusion analysis. For all of their love and support, I would also like to thank my parents, my siblings, Aaron and Liesl Hasenjager, and my partner, Brittany Kelley. And to all those individuals with whom I've jammed, talked science, or simply knocked back a pint, thanks must go to you as well! This work was supported by funding from the Animal Behavior Society, the Fisheries Society of the British Isles, the Kentucky Science and Engineering Foundation, the University of Louisville, and both the Graduate Student Council and the Biology Graduate Student Association of the University of Louisville. 


\section{ABSTRACT \\ SOCIAL DYNAMICS, NETWORK STRUCTURE, AND INFORMATION DIFFUSION IN FISH SHOALS}

Matthew Jerome Hasenjager

April 26, 2017

Animal populations are often highly structured, with individuals differing in terms of whom they interact with and how frequently they do so. The resulting pattern of relationships constitutes a population's social network. In this dissertation, I examine how environmental variation can shape social networks and influence information flow within them. In Chapter I, I review the history of social network analysis in animal behavior research, and discuss recent insights generated by network approaches in behavioral ecology. I focus on the fields of: social learning, collective behavior, animal personalities, and cooperation. Animal network studies are often criticized for a lack of replication at the network level and an over-reliance on descriptive approaches in lieu of hypothesis testing. Small, shoaling fish may provide a means to address these concerns, as manipulative experiments can be conducted on replicate social groups under captive conditions. Chapters III-V examine the impacts of environmental variation on the social networks of Trinidadian guppy (Poecilia reticulata) shoals, the social dynamics from which they emerge, and information diffusion within them. In the experiments described in Chapter III, I manipulated shoal composition in terms of within-group familiarity. Mixed shoals of familiar and unfamiliar fish exhibited greater homogeneity in network 
structure relative to other groups, which likely contributed to the rapid diffusion of foraging information observed within them. In the experiments discussed in Chapter IV, I manipulated the within-shoal mixture of personality types. In addition to impacting frequencies of partner switching and patterns of phenotypic assortment, individual- and group-level personality variation had strong effects on the initial acquisition of novel foraging information and the speed of its transmission through a group. In the experiments in Chapter V, I manipulated the ambient predation risk perceived by groups. High-risk conditions were associated with shifts in network structure consistent with attempts to minimize predation risk. High ambient risk also impeded the acquisition and subsequent transmission of foraging information, likely due to heightened neophobia and/or an increase in the perceived costs of personal sampling. I conclude in Chapter VI by considering the broader implications of my work and highlighting promising avenues for future research. 
TABLE OF CONTENTS

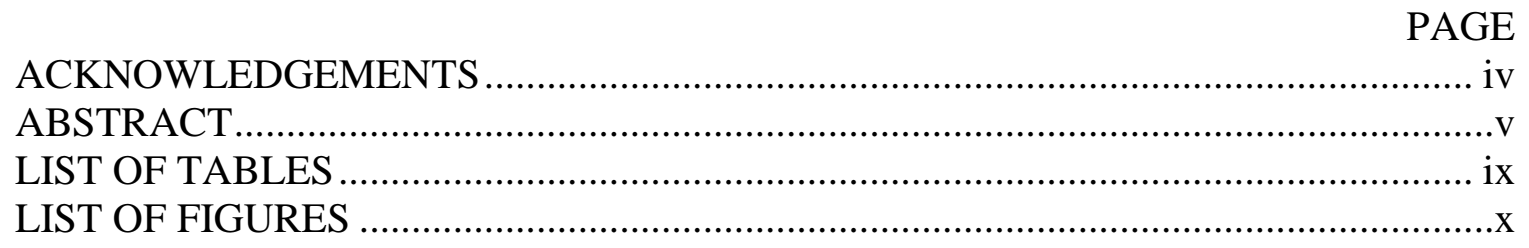

CHAPTER I: SOCIAL NETWORK ANALYSIS IN BEHAVIORAL ECOLOGY ..........1

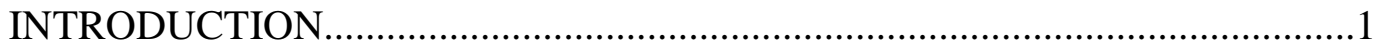

A HISTORICAL PERSPECTIVE ON THE STUDY OF ANIMAL SOCIAL STRUCTURE ......................................................................................... 10

SOCIAL NETWORK ANALYSIS AND TOPICS IN BEHAVIORAL

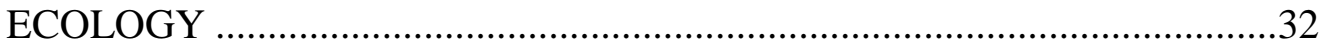

FUTURE DIRECTIONS FOR SOCIAL NETWORK ANALYSIS IN

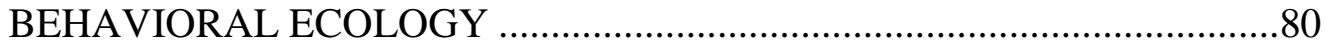

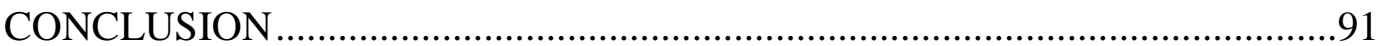

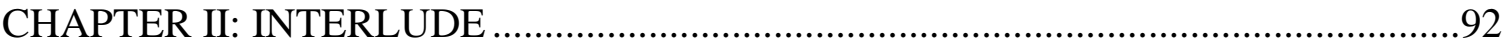

CHAPTER III: FAMILIARITY AFFECTS NETWORK STRUCTURE AND

INFORMATION FLOW IN GUPPY (POECILIA RETICULATA) SHOALS............94

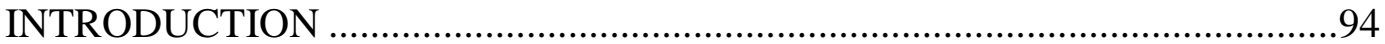

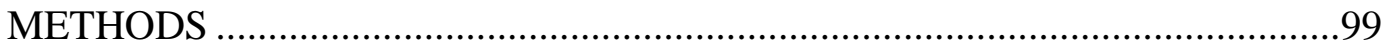

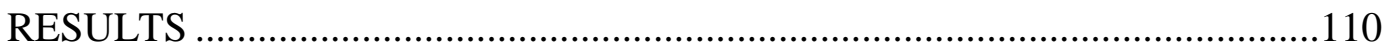

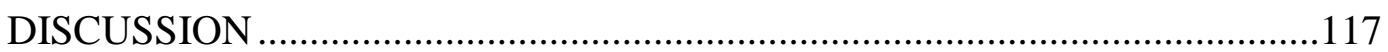

CHAPTER IV: GROUP PERSONALITY COMPOSITION SHAPES SHOALING

DYNAMICS, NETWORK STRUCTURE, AND INFORMATION FLOW ............125

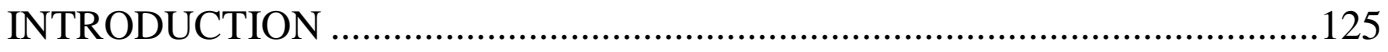

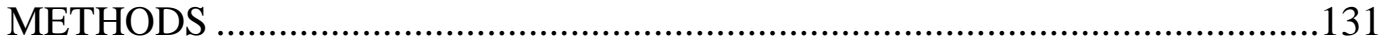

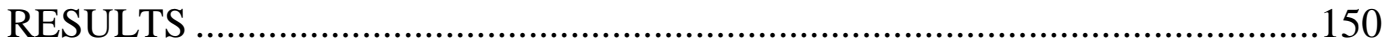

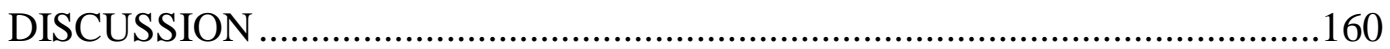


CHAPTER V: FEAR OF PREDATION SHAPES SOCIAL NETWORK

PAGE STRUCTURE AND THE ACQUISITION OF FORAGING INFORMATION ......172

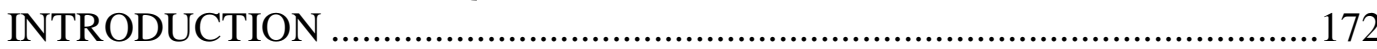

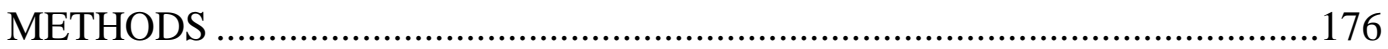

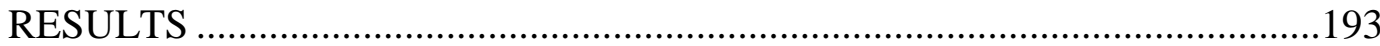

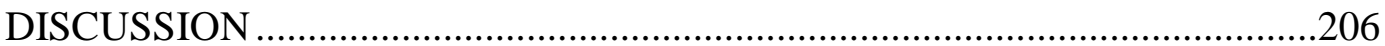

CHAPTER VI: CONCLUSION AND FUTURE DIRECTIONS .213

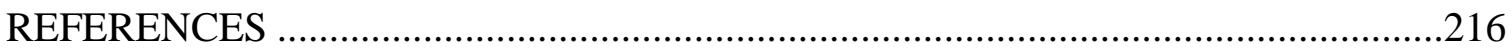

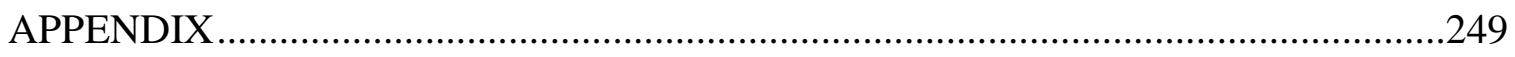

COPYRIGHT PERMISSIONS FOR CHAPTER I ………............................249

COPYRIGHT PERMISSION FOR CHAPTER II ……...................................273

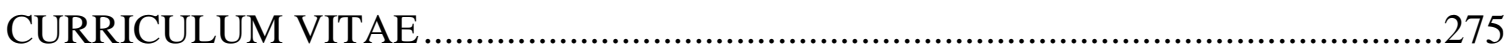




\section{LIST OF TABLES}

$\begin{array}{lll}\text { TABLE } & \text { PAGE }\end{array}$

3.1 Total support (\%) based on Akaike weights for network types and OADA variants.

3.2 Total support (\%) based on Akaike weights for social effect parameterizations ......114

3.3 Model-averaged parameter estimates, confidence intervals, and \% support .............115

4.1 (G)LMM parameter estimates, standard errors, and 95\% confidence intervals ........155

4.2 Mean assortativity coefficients $\left(r^{w}\right)$ with regards to personality type.......................157

4.3 TADA parameter estimates, standard errors, and 95\% confidence intervals ............159

5.1 Mean assortativity coefficients $\left(r^{w}\right)$ and standard errors......................................199

5.2 Parameter estimates, standard errors, and 95\% confidence intervals for the binomial generalized linear model examining the probability of a group solving the foraging task

5.3 TADA parameter estimates, standard errors, and 95\% confidence intervals for the low-risk groups

5.4 Model-averaged TADA parameter estimates, standard errors, and 95\% confidence intervals for the high-risk groups

5.5 Parameter estimates, standard errors, and 95\% confidence intervals for models examining movement through the control patch following introduction of the foraging task

5.6 Model-averaged parameter estimates, standard errors, and 95\% confidence intervals for the TADA examining movement through the control patch 


\section{LIST OF FIGURES}

FIGURE

PAGE

1.1 A simplification of Figure 1 from Hinde (1976) depicting a framework for the description of animal social structure

1.2 Sociogram of a rhesus macaque (Macaca mulatta) grooming network originally published by Sade (1965).

1.3 The four network types used in Voelkl and Noë's (2010) simulation study of social transmission in primate networks

1.4 Markov chain models of Trinidadian guppy (Poecilia reticulata) shoaling dynamics

1.5 A model of collective motion

1.6 Network structure can influence the resulting spatial structure during agent-based simulations of collective motion.

1.7 A model of collective navigation which includes social network structures

1.8 Social networks for a northern muriqui (Brachyteles hypoxanthus) population that underwent permanent group fission between the dry season in 2002 and the dry season in 2005

1.9 A social network of great tits (Parus major) based on co-occurrence at feeding stations

1.10 A model of cooperation in a structured population

1.11 Examples of triad configurations with no symmetrical relationships .84

1.12 A comparison of time-ordered versus time-aggregated networks

3.1 Test arena setup. 101

3.2 Markov chain model of shoaling behavior 105

3.3 Goodness-of-fit for the Markov chain models of shoaling behavior. 111 
3.4 Transition probabilities for Markov chain models of shoaling behavior.

4.1 Boldness assay setup.

4.2 Test arena setup

4.3 Markov chain model of shoaling behavior

4.4 Social network variants representing potential pathways of social transmission

4.5 Goodness-of-fit for the second-order Markov chain models of shoaling behavior ...152

4.6 Weighted mean transition probabilities and 95\% confidence intervals for the second-order Markov chain models

4.7 The estimated percentage of solving events that resulted from social transmission

4.8 The predicted rate at which a naïve individual will solve the foraging task at time $t$ as a function of their total connectedness to informed group members

5.1 Test arena setup

5.2 Markov chain models of shoaling behavior

5.3 Mean $( \pm S E)$ change in behavior from the pre-injection to the post-injection period for groups exposed to water and to conspecific alarm cues

5.4 Goodness-of-fit for the second-order Markov chain models of shoaling behavior ...196

5.5 Weighted mean transition probabilities and 95\% confidence intervals for the second-order Markov chain models. 


\section{CHAPTER I}

\section{SOCIAL NETWORK ANALYSIS IN BEHAVIORAL ECOLOGY ${ }^{1}$}

\section{Introduction}

Social behavior is a pervasive feature of animal life (Alexander, 1974; Wilson, 1975; Davies et al., 2012; Alcock, 2013; Dugatkin, 2013). This ubiquity suggests that an animal's social environment will often play a critical role in influencing the development and expression of their behavior, as well as its fitness outcomes (Maynard Smith, 1982; Montiglio et al., 2013; Stamps \& Groothuis, 2010). Animal social groups are often characterized by complex, dynamic, and nonrandom patterns of social relationships (Croft et al., 2008; Sih et al., 2009; Davies et al., 2012; Alcock, 2013; Dugatkin, 2013). Therefore, in order to fully understand the evolution of social behavior, these aspects of social structure must be explicitly incorporated into models of animal behavior. Over the past few decades, behavioral ecologists have become increasingly cognizant of this fact. This recognition has led to fascinating novel insights in the study of social behavior and continues to generate new, potentially very important, hypotheses that are ripe for testing. Social network theory provides both a conceptual framework and the analytical tools to explore the interplay between individual behavior, population structure, and

\footnotetext{
${ }^{1}$ This chapter was originally published in: Hasenjager, M. J., \& Dugatkin, L. A. (2015). Social network analysis in behavioral ecology. Advances in the Study of Behavior, 47, 39114. It has been reprinted here by permission of Elsevier (C) 2015.
} 
population-level processes (Croft et al., 2008). Starting in the 1930s, social network theory has been widely used in sociology to study human relationships and social organization (Moreno, 1934; Lewin, 1951; Scott, 2000). More recently, these approaches have been applied toward the study of nonhuman social systems (Croft et al., 2008; Whitehead, 2008).

Social network theory views a social group as a system of interconnected elements which are usually — though not always - individuals (Newman, 2003). A social network can be graphically depicted as a collection of nodes, where each node represents an individual within the group. Social interactions or associations between two individuals are denoted by an edge connecting their two nodes together. Nodes can be assigned attributes - e.g., sex, body size, personality type - corresponding to the individual they represent. Edges, too, can vary in a number of properties. For example, edges can be weighted to indicate the relative frequency or intensity of a relationship, such as how often two individuals copulated, or directed to indicate asymmetric interactions - e.g., individual A groomed B, but not vice versa. The pattern of edges connecting nodes together, combined with the attributes possessed by the nodes and edges, makes up a group's social network.

Social network analysis (SNA) provides researchers with a wide variety of tools to explore different aspects of network dynamics, structure, and function. The structure of a social network can be described using a multitude of quantitative network measures that capture different aspects of social structure at the level of the dyad, the individual, and the population (see examples in: Box 1; Croft et al., 2008; Wey et al., 2008; Whitehead, 2008). Networks often possess emergent properties arising from the complex ways in 


\section{Box 1 Terminology of Social Network Analysis}

Social network theory views a social group as a system of interconnected individuals (Newman, 2003). Social network analysis (SNA) uses a variety of toolse.g., visualization, descriptive measures, modeling, and simulations - to explore the dynamics that form a social network, the structure of that network, and the consequences of that structure for processes occurring over the network and the behavior of individuals within it. These analyses can scale from the individual-level up to that of the population.

A social network can be visually represented as a series of nodes (also: vertices) representing individuals connected by lines (also: edges, ties) representing social relationships between two connected individuals. This visual representation is also known as a sociogram or graph. Ties can be unweighted (also: binary), where a tie between two nodes simply indicates the presence of a relationship (e.g., grooming), or weighted, where ties indicate the strength or frequency of an interaction (e.g., the number of times grooming occurred). Ties can also be bidirectional for symmetrical or reciprocated interactions, as is often the case for proximity, or they can be directional when interactions are asymmetrical or unreciprocated, such as if individual A groomed, but was never groomed by, individual B.

In addition to visual representation, a social network can also be represented as a sociomatrix, defined as the matrix of association or interaction measures between each pair of individuals in the population. Most quantitative network analyses are performed using this matrix.

Throughout this review, we use the term social structure to refer to the quality, content, and patterning of social relationships within a population (Hinde, 1976). The 
population will be defined as the collection of potentially interacting individuals on which a particular social network is based. A community is a set of nodes that are more densely interconnected to one another than they are to the wider network. The extent to which communities play an important role in dividing up a population can be assessed via Newman's (2004) modularity measure, which takes the difference between the proportion of total weights or edges connecting individuals within communities and the proportion expected if individuals associated at random.

A variety of network measures are available to describe different aspects of an individual's pattern of connectedness. Often, individual measures can be averaged across all individuals in the population — or across a class of individual— to provide populationor class-wide measures of social structure. Below, we introduce several commonly used network metrics and provide references in which they have been applied and/or where formulas for their calculation can be found.

Degree: the total number of connections a node has. In-degree and out-degree can be quantified for directional ties. For example, an individual's in-degree could be the number of social partners that have groomed it, while its out-degree would be the number of social partners it has groomed. Degree provides a measure of how well connected an individual is in its network - as well as its potential importance to overall network structure-based on its direct social partners.

Strength: the total weight of all ties connected to a node. In-strength and outstrength can be calculated for directional, weighted ties. Strength is the corresponding measure for weighted networks that degree is for binary ones. Strength also serves as a measure of gregariousness (Whitehead, 2008). 
Clustering Coefficient: measures the extent to which a node's network neighbors are also connected with one another. The clustering coefficient averaged over the whole network provides a measure of how cliquish a network is; networks with high clustering are made up of highly interconnected social units (see Newman, 2003; Holme et al., 2007; Whitehead, 2008).

Eigenvector Centrality: a measure of how well connected a node is, taking into account not only the number and strength of direct connections, but also how well connected that node's neighbors are. Unlike degree or strength, eigenvector centrality also takes indirect connections into account. Computationally, eigenvector centrality is obtained from the first eigenvector of the sociomatrix (see Newman, 2004; Whitehead, 2008).

Reach: a measure of indirect connectedness; in a binary network, it measures the number of nodes $n$ or fewer steps away from the focal node. See Whitehead (2008) for an example of how reach can be calculated for a weighted network. This measure might be particularly useful when a researcher is interested in the possibility of the spread of a behavioral trait or a type of interaction-e.g., agonistic behavior between individuals A and B causes B to direct agonistic behavior toward individual C (see Flack et al., 2006; Whitehead, 2008).

Path Length: the number of edges on the shortest pathway between two individuals. Path length measures how well connected two nodes are with each other.

Betweenness: the number of shortest path lengths between pairs of nodes in the network that pass through the focal node. Individuals that have high betweenness link together many individuals in the network and can therefore have particularly important 
effects on the flow of information, disease, or resources through a population. For example, imagine two clusters of individuals where the only connection between these clusters passes through a single intermediate individual. If a novel behavior arises in one cluster and spreads via social learning, the only way for that trait to reach the other cluster is through that intermediate individual (see Freeman, 1979; Lusseau \& Newman, 2004; Whitehead, 2008).

Information Centrality: measures a similar property as betweenness, but also takes into account longer pathways weighted by the inverse of their length (see Stephenson \& Zelen, 1989; McDonald, 2007; Vital \& Martins, 2011, 2013).

By viewing social groups as a system of interconnected nodes, social network theory highlights the potential for emergent properties to arise at the population level as a consequence of the complex patterns of relationships between individuals. Emergent properties are not predictable by considering each contributing factor in isolation from one another (Bradbury \& Vehrencamp, 2011). In network terms, the structural properties of a network usually cannot be assessed by measuring the dyadic relationships of its constituent members in isolation. Only when these same relationships are allowed to interact with one another in the context of the whole population are we able to properly assess the structure and function of a network. Examples of emergent network properties include: population-wide resilience to loss of members (e.g., Lusseau, 2003), the formation of stable dominance hierarchies (e.g., Shizuka \& McDonald, 2012), multitiered social structures (e.g., VanderWaal, Wang, et al., 2014), and the rate at which socially learned behaviors spread through a population (e.g., Whitehead \& Lusseau, 2012; Aplin et al., 2015). 
which nodes can interact with one another (Bradbury \& Vehrencamp, 2011).

Mathematicians have constructed several types of network models to better understand these emergent properties, their function in real-world networks, and how these networks form and evolve over time (Newman, 2003). These models can then be tapped by social network analysts for a number of purposes. For example, observed network measures can be compared to those generated from a simulated network to identify significant departures from null expectations, thereby revealing potentially important aspects of a population's social organization (Croft et al., 2008). Network modeling can also be used to determine the causal factors-e.g., individual behavior, environmental conditionsthat drive observed network structure (Newman, 2003; Pinter-Wollman et al., 2014). Furthermore, a social network provides the substrate upon which population-level processes - e.g., disease transmission, information flow, or the emergence and maintenance of cooperation - may play out. Understanding the dynamics and structure of a population's social network provides us with predictive power with respect to these processes and can enhance our understanding of how social organization influences individual behavior (e.g., Croft et al., 2006; Hoppitt \& Laland, 2013; Wilson et al., 2014). SNA offers several advantages to behavioral ecologists when combined with more traditional methods of studying social structure and behavior. For one, SNA provides a holistic framework that directly links individual behavior to population structure. By population, we refer to a set of potentially interacting individuals in which the majority of interactions are among its members (Whitehead, 2008); in practical terms, the population refers to all the nodes making up a given social network. The ability of SNA to integrate individual behavior and population structure allows for a more 
sophisticated exploration of questions at both levels; many behaviors can only be fully understood when placed within the social context of the entire population. For example, the spread of social information, diseases, or parasites through a population depends not only on whom an individual directly interacts with, but also with whom their social partners interact (Godfrey et al., 2009; Hoppitt \& Laland, 2013; VanderWaal, Atwill, et al., 2014).

SNA also provides behavioral ecologists with a complex and detailed view of social structure applicable to a myriad of species and behavioral milieus. In this review, we define social structure as the nature, quality, and patterning of social relationships within a population, where a relationship summarizes the content, quality, and patterning of interactions between two individuals (Hinde, 1976); following Whitehead (2008), we use the terms social structure, social organization, social system, and society interchangeably. SNA incorporates information on individual behavioral variation and offers a wealth of network measures which provide an objective means of quantifying a population's social structure. This approach can complement conventional methods of describing animal societies - e.g., via group size, demography, mating system, or division of labor- that often downplay the variation and complexity of intragroup relationships or are only useful for specific taxonomic subgroups (Wilson, 1975; Wey et al., 2008; Whitehead, 2008). Furthermore, these network descriptors can facilitate comparative studies between populations and species to better understand how social structure and behavior is shaped by ecology and evolutionary history.

In addition to casting new light on old problems, a social network approach can highlight previously unconsidered or neglected social processes. If a social network is a 
system of interconnected nodes, then the potential exists for interactions between those nodes to involve nonlinear elements-e.g., competition, interference, or cooperationwhich, in turn, may generate emergent properties (Couzin et al., 2002; Sumpter, 2006; Bradbury \& Vehrencamp, 2011). The potential for animal social networks to facilitate emergent social phenomena has been traditionally underappreciated (Bradbury \& Vehrencamp, 2014). The establishment of linear dominance hierarchies (e.g., Shizuka \& McDonald, 2012), collective decision-making (e.g., Sueur et al., 2012), and the collective motion of animal groups (Bode et al., 2011a) are all classic examples of emergent social processes; in each case, our understanding of the phenomenon has been enhanced by adopting a network-based approach.

Network theory is being simultaneously developed in a number of fields, including statistical physics, sociology, molecular biology, and computer science. As a result, the field is changing at a rapid pace, with concepts, approaches, and measures developed in one context often finding use in another. While not all developments canor should — be applied toward the study of animal societies (James et al., 2009), this rush of novel ideas from outside sources is sure to enrich behavioral ecology.

Our goal in this review is threefold. First, we will trace the history of the study of nonhuman social structure from early ethological ideas to modern social network theory. In so doing, we shall see that the fundamental questions and topics dealt with by social analyses have changed very little over time. However, the development of new conceptual frameworks and analytical techniques, as well as extensive cross-pollination from other disciplines, has allowed behavioral biologists to increasingly embrace the complexities seen in the natural world. Next, we will outline the concepts behind modern 
social network theory and discuss some of the new insights it has provided behavioral ecologists over the past decade. Here, we focus on social learning, collective movement and decision-making, animal personalities, and animal cooperation. While this by no means represents an exhaustive list of the potential topics to which SNA has been-or can be-applied, the above behaviors and phenomena possess many features-e.g., indirect effects, dependence on population structure, emergent properties - that networkbased approaches are especially well suited to handle. Third, and finally, we will highlight intriguing new avenues of research as advancing technology and statistical methods allow researchers to address more nuanced questions regarding social behavior than ever before.

Just as SNA in behavioral ecology developed from earlier approaches to studying population structure and social behavior, SNA itself is evolving. Indeed, SNA has experienced an influx of new ideas and applications over the past decade, as well as generated a wealth of novel insights. Since it has been several years since a number of reviews on the subject (Krause et al., 2007; Croft et al., 2008; Wey et al., 2008; Whitehead, 2008; Sih et al., 2009), we feel the time is right to revisit it and review many of its recent developments. In this way, we hope to serve as a conceptual introduction to SNA for behavioral ecologists and a source of inspiration for future research.

\section{A Historical Perspective on the Study of Animal Social Structure}

Drawing on developments from ethology, sociology, primatology, statistical physics, and behavioral ecology, the history of SNA in nonhuman systems is a rich one. While a comprehensive treatment is not possible here, we have distilled what we feel are the major developments leading up to the application of modern network analysis in 
behavioral ecology. The introduction to Whitehead (2008) and the review by Brent et al. (2011) provide more on this subject, with the latter dealing specifically with the study of nonhuman primate social structure. We do not review here the extensive sociological literature on network analysis except where it explicitly intersects with our primary objective-i.e., examining the evolution of network analysis in nonhuman systems. Interested readers should instead refer to several excellent treatments of that subject (Wasserman \& Faust, 1994; Scott, 2000; Freeman, 2004; Borgatti et al., 2009; Scott \& Carrington, 2011).

\subsection{Early Approaches}

The evolutionary and ecological importance of animal social structure was formally recognized as early as the late nineteenth century (Crook, 1970; Whitehead, 2008). In 1878, Espinas proposed that animal societies were not simply random assemblages of individuals, but rather possessed structure and persisted as distinct entities over time (Espinas, 1878). Espinas argued that variation in animal social structures was related to ecological conditions rather than phylogenetic history. For example, the territories of carnivorous or piscivorous birds were often more defined and better defended than those of other avian species, but these territorial boundaries would break down during periods of high resource abundance. Espinas further argued that animal societies possessed emergent, group-level properties that arose from the complex web of social interactions within a population. He even suggested that animal societies could be influenced by natural selection and evolve as entities in their own right.

Petrucci $(1905,1906)$ discussed animal territories and social organization in relation to individual, familial, and societal requirements, though he was careful to note 
that lower levels need not be present for higher levels to be in place-i.e., a society can form independently of familial concerns, while a family can be considered a social group in its own right. Like Espinas, Petrucci noted a correlation between environmental conditions and animal social structure, suggesting selection pressures played a role in shaping social organization. These early ideas lay fallow for some time before being rediscovered in the mid-twentieth century, in part due to neither author being biologists by trade, as well as the fact that contemporary biologists of the time were not particularly interested in such questions (Crook, 1970).

The first comprehensive attempt to place nonhuman social behavior within a larger conceptual framework came with the birth of ethology. For the most part, discrete social behaviors were believed to be innate and under control of special "centers" within the brain (Tinbergen, 1953). Over time, action-specific energy built up in these centers, requiring release which was provided by the presence or behavior of conspecifics. Lorenz (1937) likened the phenomenon to that of a lock and key. Natural selection shaped species to behaviorally respond in appropriate ways to unique combinations of stimuli (i.e., the "key") to which their brain was attuned (i.e., an innate perceptory pattern or the "lock"). While this system was believed to apply to any stimulus-response relationship, when the releasing stimulus involved a conspecific_-i.e., a kumpan in Lorenz's terminology — signaler-receiver coevolution was possible over evolutionary time. This coevolutionary process could then give rise to specialized morphological structures and stereotyped motor patterns as seen, for example, in many avian courtship rituals. These morphologies and behaviors presumably evolved for the explicit purpose of influencing conspecifics. 
To an early ethologist, social organization was simply the sum of the innate stimulus-response relationships corresponding to conspecifics (Tinbergen, 1953). Little consideration was given at the time to questions of group composition and how relationships were patterned within a group, nor how these structural elements might influence social behavior both between group members and over the course of an individual's life. Rather, dyadic interactions had been primarily studied as isolated phenomena detached from their wider social environment (Beer, 1976; Hinde, 1982). Intra- and interindividual variation in behavior was downplayed; instead, innate, speciesspecific stereotyped behavior patterns were emphasized (Hinde, 1982).

This situation began to change in the 1950 s as researchers started to question ethology's highly mechanistic explanations for behavior, as well as its tendency to neglect the full gamut of factors that could influence behavioral development and expression (Hinde, 1959; Kennedy, 1954; Lehrman, 1953). More explicit consideration was given to how genetic, ecological, and social factors interacted to produce variation in social structures and behavior (McBride, 1964; Lack, 1968). This transition came about in part due to recognition of significant intraspecies variability in primate social behavior that was not well explained by a system of innate, inherited releasing mechanisms (Crook, 1970). For example, harkening back to ideas raised by Espinas, studies found that vervet monkeys (Chlorocebus pygerythrus) living on a small island with rich food sources demonstrated territorial behavior not observed in vervet groups living in larger areas with sparser resources (Crook \& Gartlan, 1966). The ecological conditions experienced by a group were an important determinant of social structure, while the ontogeny and expression of an individual's social behavior were critically influenced by 
both the ecological and social contexts experienced by that individual (McBride, 1964; Crook, 1970).

\subsection{Searching for a Conceptual Framework}

As interest in describing and comparing animal social structures between and within species grew, researchers recognized the need for a common framework within which they could work. C. R. Carpenter (1942a, 1942b, 1952) was one of the first to consider nonhuman primate social structure in a comparative sense. He developed species-specific models that described the spatial arrangement of individuals as determined by individual- and class-level patterns of affiliation and avoidance, though his work garnered little interest at the time (Sade, 1972). Drawing from prior classification schemes of social behavior in both sociology and ethology (Scott, 1945; Bales, 1951), Thompson (1958) sketched out a potential comparative framework of social structure that distinguished between social interactions that had either a positive or negative influence on group unity. Layered atop of this would be characteristics of the actors and recipients, such as sex or kinship, whether the interactions involved in- or out-group members, and their function (e.g., foraging, reproduction). The sum of these interactions formed the structure of a group, which could be characterized by: (i) the number of group members, (ii) their density, (iii) their cohesiveness - that is, the physical proximity of group members, (iv) the coordination exhibited among members when carrying out various tasks, and (v) group stability and permeability.

Noting the failure of earlier attempts to classify animal societies as resulting from an overly reductionist approach or a lack of generality by focusing too closely on taxonspecific social traits—e.g., eusociality, mating system, life cycles-E. O. Wilson 
advocated that researchers instead focus on social qualities that could be universally applied toward any study system (Wilson, 1975). He expanded on Thompson's (1958) list, devising a set of 10 qualities by which researchers could describe social structure: (i) group size, (ii) demography, (iii) cohesiveness, (iv) amount and patterns of connectedness, (v) permeability, (vi) compartmentalization - that is, the extent to which subgroups act as one unit, (vii) role differentiation, (viii) coordination of behavior, (ix) information flow, and (x) fraction of time devoted to social behavior. Today, SNA allows researchers to study many of these qualities-e. g., cohesiveness, connectedness, compartmentalization, behavioral coordination, and information flow-under one integrated framework (Whitehead, 2008).

Behavioral ecologists, excluding those that worked with primates, were slow to adopt many of these social qualities (Whitehead, 2008); nonprimate social structures were primarily described by group size and demography (e.g., Jarman, 1974; Brosset, 1976). This stemmed both from a lack of analytical tools, as well as the misguided assumption that only primates had social systems that were sufficiently complex-e.g., involving individual recognition — to warrant such studies (Whitehead, 1997, 2008). Even cognitively advanced and highly social animals, such as cetaceans, were dismissed as having a relatively simple social organization (Gaskin, 1982). Primatologists, however, forged ahead with a number of approaches to describing, classifying, and comparing social systems (Silk, 2007; Brent et al., 2011).

Because sociologists also study primates_albeit usually focusing solely on humans - it is not all that surprising that there is a rich tradition of importing methods developed in sociology to study nonhuman primate social structure (Roney \& 
Maestripieri, 2003). A particularly profitable import from sociology was the sociometric approach. Developed in the 1930s, sociometry sought to quantitatively describe the structure of human groups, and the positions of individuals within those groups, through application of mathematical graph theory (Moreno, 1934; Lewin, 1951). The pattern of social relationships between group members determined overall social structure and could be depicted as a set of nodes connected by edges-i.e., a sociogram (Box 1) (Moreno, 1934; Scott, 2000). Sociometric analyses usually took the form of creating matrices that quantified some type of interaction between each pair of individuals in a group and used quantitative measures to describe the resulting pattern of social relationships. These analyses could be applied toward potentially any type of interaction, such as aggression, trade, affiliation, or communication.

Drawing from these sociometric ideas, ethologist R. A. Hinde (1976) sought to provide a unifying conceptual framework for studies of primate social structure, though he also recognized its potential utility for nonprimate animals as well. The framework he proposed had three levels: interactions, relationships, and social structure, each of which influenced, and was influenced by, the other two levels (Figure 1.1). Interactions involve specific instances in which two individuals do something together or in which an individual directs an action toward another individual—e.g., two baboons grooming one another or two fish shoaling together. Repeated interactions over time between two individuals form the basis of their relationship. Description of a relationship includes not only what two individuals do together, but how those interactions are patterned-e.g., the frequencies and timing of interactions, as well as the effect one type of interaction can have on another. Taken together, the nature and patterns of relationships within a group 


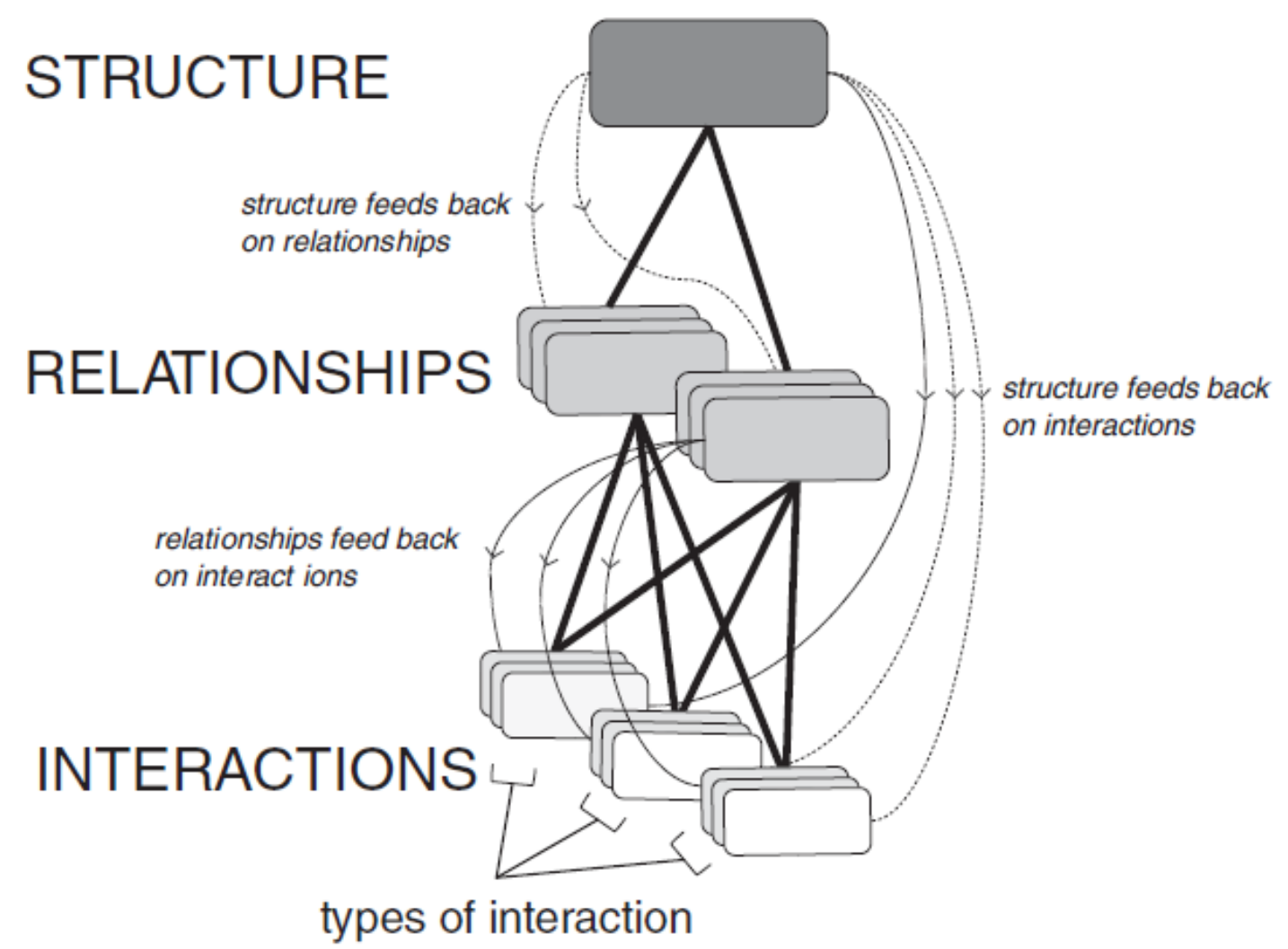

Figure 1.1 A simplification of Figure 1 from Hinde (1976) depicting a framework for the description of animal social structure. Successive interactions between two individuals make up their relationship, while the pattern of relationships within a population determines social structure. Feedbacks can occur between each level; for example, structure can influence the types of interactions likely to occur between two individuals. The relationship of two individuals accounts for all types of social interactions that have occurred between them—e.g., grooming, copulation, agonistic behavior-as well as the frequency and temporal patterning of those interactions. Reprinted with permission from: Brent et al. (2011). Social network analysis in the study of nonhuman primates: a historical perspective. American Journal of Primatology, 73, 720-730. Copyright @ 2011 John Wiley and Sons. 
make up the social structure of that group. New properties emerge at each of Hinde's three levels - interactions, relationships, and structure - that are not present in the component units making up the lower levels. For example, the nature of an interaction results from the behavior of both individuals involved, each of which can act in a variety of ways depending on the identity of their partner. Hinde also discussed how various factors, such as kinship, sex, or age, might be expected to influence relationship patterns. By clarifying the links between interactions, relationships, and the emergence of social structure, Hinde (1976) provided a conceptual framework that linked individual behavior to population structure and vice versa. In doing so, he underscored the importance of studying social behavior within the context of the whole population, as population structure could feed back to influence the nature of social interactions and relationships. Hinde's framework has proven itself to be widely applicable (e.g., Whitehead, 2008) and it was influential in prompting further development of sociometric approaches in nonhuman animals_-e.g., block models (Pearl \& Schulman, 1983)—as well as other forms of social analyses - e.g., ordination methods and lagged association rates (Kappeler, 1993; Whitehead, 1997; Whitehead \& Dufault, 1999). Sociometry in particular was an important precursor to modern SNA (Brent et al., 2011), though it had some crucial limitations which we highlight below.

\subsection{The Development of Sociometric Approaches in Primates}

Sociometric approaches such as those advocated by Hinde (1976) were initially applied primarily to nonhuman primates and proved a fertile ground for researchers. For example, presenting social data as a sociogram allowed important, and sometimes nonintuitive, features of social structure to be highlighted in a much more accessible 
format as compared to data matrices. An early application of this technique to primate research was Sade's (1965) depiction of rhesus macaque (Macaca mulatta) grooming relationships as a network of nodes connected by lines indicating the direction and frequency of grooming interactions between two individuals (Figure 1.2). Sociograms have been widely used to depict primate social relationships including: grooming (e.g., Soczka, 1974; Seyfarth, 1976, 1977; Cheney, 1978a; Fairbanks, 1980; Hanby, 1980b; Seyfarth, 1980; Pearl \& Schulman, 1983; Mitani, 1986; Chepko-Sade et al., 1989; Nakagawa, 1992), proximity (Fairbanks, 1980; Hanby, 1980a; Seyfarth, 1980; Nakagawa, 1992), agonism (Hanby, 1980b; Pearl \& Schulman, 1983), play (Soczka, 1974; Cheney, 1978b; Pearl \& Schulman, 1983), and copulations (Cheney, 1978a; Pearl \& Schulman, 1983). While there is a limit to the amount of usable information that can be effectively conveyed in a sociogram, they remain an invaluable graphical tool for SNA. As an example of the utility of the sociometric approach, we turn to the rich history of studies on primate allogrooming (e.g., Sade, 1965; Kummer, 1968; Seyfarth, 1977). The structuring of grooming relationships was of particular interest to primatologists both because of the relatively high frequency at which grooming interactions occurred relative to other primate social behaviors and because other important behaviors and processes were suspected to be influenced by this structuree.g., coalitionary support and the likelihood of receiving aggression (Rhine, 1973; Seyfarth, 1980; Seyfarth \& Cheney, 1984). These studies revealed that grooming interactions were nonrandomly distributed within primate groups and that this structure was driven by a variety of social factors. For example, fewer than $15 \%$ of the possible dyads accounted for $62 \%$ of the grooming interactions in one group of rhesus macaques 


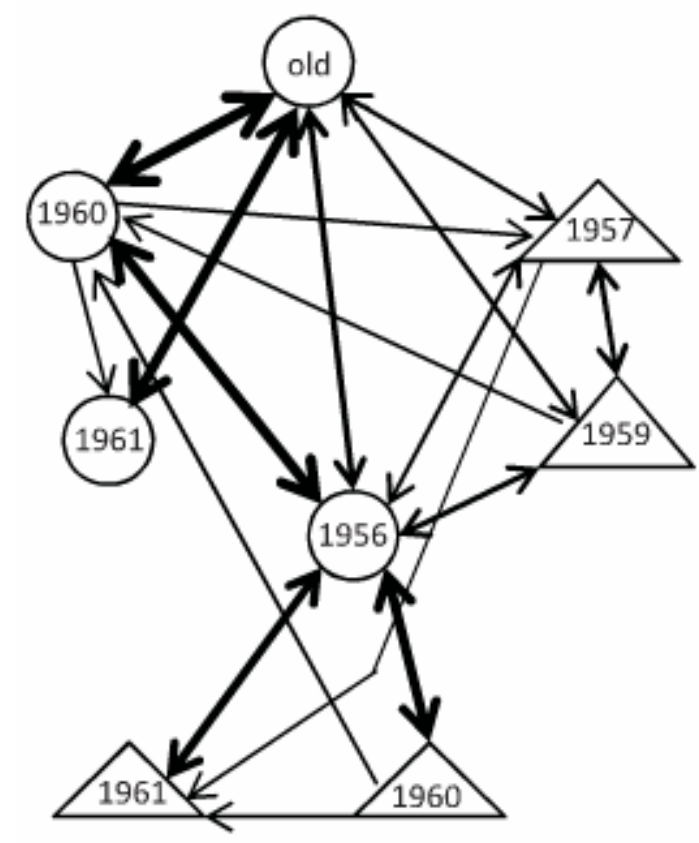

Figure 1.2 Sociogram of a rhesus macaque (Macaca mulatta) grooming network originally published by Sade (1965). Circles indicate females and triangles represent males. The lines between individuals indicate that grooming has occurred between these individuals; the thickness of the line is proportional to the frequency of grooming interactions. The arrows depict the direction of grooming-e.g., individual 1960 groomed, but was not groomed by, individual 1961. Reprinted with permission from: Brent et al. (2011). Social network analysis in the study of nonhuman primates: a historical perspective. American Journal of Primatology, 73, 720-730. Copyright (C) 2011 John Wiley and Sons. 
(M. mulatta), indicating a highly structured grooming network based on kinship (Sade, 1965). High-ranking females were preferred grooming recipients relative to low-ranking females in several species, including Chacma baboons (Papio ursinus) (Cheney, 1978a; Seyfarth, 1976), stump-tailed macaques (Macaca arctoides) (Rhine, 1973), and vervet monkeys (C. pygerythrus) (Seyfarth, 1980). However, females tended to instead groom individuals of adjacent social rank to themselves due to a number of influences-e.g., constraints imposed by higher-ranking individuals, kin-based preferences (Sade, 1965, 1972; Seyfarth, 1976; Cheney, 1978a; Seyfarth, 1980)—-thereby demonstrating how studying dyadic relationships in isolation from the larger social environment can be misleading. Furthermore, sudden increases in grooming received were often observed for lactating females with infants, highlighting the dynamic elements of social structure (e.g., Seyfarth, 1976; Cheney, 1978a; Seyfarth, 1980).

Most sociometric analyses either focused on only one type of interaction (e.g., grooming) or examined multiple behaviors independent of one another. However, animals are embedded simultaneously within multiple networks, each of which might exert influence on the others. Pearl and Schulman (1983) attempted to combine multiple social networks into a single network through their application of block models to two social groups of rhesus macaques (M. mulatta). Sociomatrices for grooming, play, mating behaviors, proximity, threat displays, and fear grimaces were constructed and combined into one large matrix. Macaques were then partitioned into "blocks" such that the relationships individuals within a block share with those outside of their block are largely similar to one another. The behavioral profiles of these blocks were then compared within and between groups. A comparison of how different types of interaction related to one 
another-for example, the relationship between proximity and play networks — suggested common factors influenced social structure in both groups despite dramatically different demographic profiles within, and ecological conditions experienced by, each population. One group was made up of about 25 wild individuals in the mountains of Pakistan, while the other was a large, free-ranging group containing about 100 individuals on the Caribbean island of Cayo Santiago. While these early block model studies were useful for considering multiple network types simultaneously, as well as potentially facilitating comparative studies of social structure, they tended to discard a great deal of information regarding an individual's network position that was of great interest to behavioral biologists. Block modeling has not been widely used in behavioral ecology, though it has seen continued use and development in other fields, including molecular biology (e.g., Wang \& Qian, 2014) and sociology (e.g., Žiberna, 2014).

Even though it lacked a robust, quantitative methodology, sociometric analyses in primates presaged modern SNA in many ways. As in SNA, these early studies constructed a representation of social structure based on repeated interactions between group members, used numerical measures to describe this structure, and could graphically depict structural patterns using a sociogram. Also like SNA, sociometry sought to understand the reciprocal interplay between individual behavior and overall group structure (e.g., Sade, 1972; Hinde, 1976; Seyfarth, 1977; Hanby, 1980b; Sade et al., 1988).

Sociometric studies were hamstrung by a lack of computational power, as well as by methodological issues (Brent et al., 2011). While Hinde (1976) had provided a useful conceptual framework for visualizing social structure, tools for quantitatively analyzing 
this structure lagged behind (Pearl \& Schulman, 1983). Some network measures such as degree (i.e., the number of social partners an individual has) and strength (i.e., the frequency of interaction) were easily calculated by hand and were frequently used. Utilization of most other network metrics, however, had to await greater availability of computing power (though see: Sade, 1972; Kaplan \& Zucker, 1980; Sade et al., 1988). Methods for assessing the statistical significance of nonindependent, relational data, such as is used in network studies, were infrequently applied (Sade \& Dow, 1994). Comparing social structures between groups, populations, and species remained fraught with challenge (Sade, 1972; Chepko-Sade et al., 1989; Whitehead, 1997). Further, sociomatrices and sociograms represented a static image of a network that in reality was likely to be constantly changing as a result of environmental, social, and demographic factors (Sade, 1965; Hanby, 1980b). While many of these issues remain challenges for SNA, progress has since been made on several of them.

A more important difference between modern SNA and these early sociometric studies is that SNA goes beyond simply describing and depicting network structure: SNA attempts to understand how that structure forms, what properties it might possess, and its function in ecological and evolutionary processes (e.g., Pinter-Wollman et al., 2014). For example, modern SNA might ask how network structure influences the flow of information through the network or how it impacts the use of behavioral strategies in the population. Furthermore, SNA emphasizes the potential for social structure to possess emergent properties, such as resilience in terms of network structure and function when faced with removal of individuals from the population (e.g., Lusseau, 2003). In these ways, as well as others, modern SNA encompasses much of the sociometric approach, 
but also amends it significantly by adding new concepts, questions, and techniques.

Before we discuss the use of modern SNA in behavioral ecology, however, we first turn to some of the important contributions to social analyses made by nonprimatologists.

\subsection{The Study of Social Structure Embraces Nonprimates}

In the 1980s and 1990s, behavioral ecologists (many of whom were trained by primatologists) began to apply sociometric methods and related approaches to nonprimate species, including: ungulates (Clutton-Brock et al., 1982; Le Pendu et al., 1995), cetaceans (Bigg et al., 1990; Connor et al., 1992), and birds (Ekman, 1979; Myers, 1983). Contrary to earlier claims, it rapidly became apparent that nonprimates also possessed a great deal of complexity in their social interactions and organization. For example, male bottlenose dolphins (Tursiops spp.) were demonstrated to preferentially associate with one or two other males, forming long-term alliances through which members gained increased access to females (Connor et al., 1992). In some instances, two of these alliances would even cooperate, forming a superalliance that facilitated joint theft of a female from another alliance (Connor et al., 1992; Connor et al., 1999). Many ungulate species were shown to possess seasonal variability in social organization, as well as nonrandom, preferential associations between individuals based on age and sex (e.g., Underwood, 1981; Le Pendu et al., 1995). By expanding work to nonprimate social systems, behavioral ecologists were able to study a wider variety of life histories, mating systems, demographics, ecological contexts, and other factors relevant to social structure than they could previously. How this variation translated into variation in social structure - and how that, in turn, influenced the fitness outcomes of social behaviorprovided innumerable avenues for fascinating research. 
Analytical techniques were developed which could deal both with the much larger population sizes present in many nonprimate species relative to primate populations, as well as interaction or association data that were often much sparser than that available in primate studies (Whitehead \& Dufault, 1999). For example, lagged association rates and other similar techniques allowed researchers to describe the temporal patterning of social relationships in a population. These temporal patterns are an important aspect of social structure, as the same average interaction rate between two individuals can have quite different interpretations if interactions persist at a low, but stable, frequency over time as compared to a high initial interaction frequency that quickly falls to zero. Myers (1983) calculated the likelihood of sanderling (Calidris alba) pairs remaining together over time intervals of hours, days, months, and years, finding that associations broke up quickly as birds moved throughout the foraging area and that associations did not persist over longer time periods. Underwood (1981) created what were basically survivor curves for the length of time two animals remained associated with one another, and found that individual eland (Taurotragus oryx) were often found together on consecutive days, but associations tended to quickly deteriorate after that.

These two approaches were further developed by Whitehead $(1995,1997)$ as the lagged association rate which calculates the probability of association $\tau$ time units since an earlier known association between two individuals. While a significant amount of data are needed to confidently characterize the temporal relationship for a particular dyad, the lagged association rate can be generalized over a class of individual, a community, or an entire population (Whitehead, 2008). In doing so, it provides a powerful approach that can be used even for species where social behaviors are difficult to observe and specific 
individuals are encountered rarely and opportunistically-e.g., sperm whales (Physeter macrocephalus) (Whitehead, 1995, 2008).

\subsection{The Advent of Modern Social Network Analysis in Nonhuman Systems}

It is difficult to draw a firm line dividing SNA from earlier sociometric methods. While some authors appear to see a clear delineation between the work we have described above and SNA (Whitehead, 2008), others—e.g., Croft et al. (2008)—see SNA as further development of these early approaches that simultaneously embraces concepts, techniques, and methodologies from a wide range of disciplines in which network approaches have been applied, including sociology (Moreno, 1934; Homans, 1951; Wasserman \& Faust, 1994; Hanneman \& Riddle, 2005), business (Levine, 1972), economics (Burt, 1988), ecology (Harary, 1961; Solé \& Montoya, 2001), physics (Newman, 2003, 2004), and molecular biology (Rausher et al., 1999; MacCarthy et al., 2003; Kollmann et al., 2005). While this can occasionally lead to misapplication of concepts and analyses that are not relevant when applied to animal groups (see James et al., 2009), it also allows researchers to explore social structure from a number of angles in order to better identify both the causal factors that drive observed patterns and the consequences of those patterns (Croft et al., 2008).

The main conceptual difference between early network approaches, such as sociometry, and modern SNA is that the latter emphasizes viewing a social network as a system of interconnected nodes that has the potential to generate complex properties and outcomes (Newman, 2003; Bradbury \& Vehrencamp, 2014). Modern SNA does not just use descriptive measures to quantify individual- and population-level social structure, though that certainly remains an important part (Croft et al., 2008): It also seeks to 
understand the functional consequences of different types of network structure. SNA identifies emergent structural properties that arise from complex patterns of social relationships and explores how these properties influence individual behavior occurring within the network and social processes playing out over it (Newman, 2003). SNA also tries to understand the ecological factors and underlying social dynamics that result in the emergence of particular structural elements (e.g., Barabási \& Albert, 1999; Wilson et al., 2014). To accomplish these goals, modern SNA has a wide array of tools at its disposal, including: descriptive measures, network models, simulations, and comparative approaches (e.g., Pinter-Wollman et al., 2014). While we will define concepts and terminology in the body of the text as they are used, readers can also refer to Box 1 where we provide definitions and corresponding references.

An early study explicitly applying elements of modern social network theory to nonhuman animals was Maryanski's (1987) work on gorilla (Gorilla gorilla) and chimpanzee (Pan troglodytes) social organization. She used data from the literature to characterize the average relationship between different age-sex classes in chimpanzee and gorilla groups in terms of strong or weak ties. Using this approach, Maryanski demonstrated similarities between chimpanzee and gorilla social structure in that most individuals maintained a handful of strong social ties, but the majority of their social connections, though fairly weak, were distributed throughout the regional population. This resulted in a large amount of interconnectedness at the population level which could have facilitated the movement of individuals and the transmission of social information throughout the population (e.g., Granovetter, 1973). Maryanski contrasted this with baboon (Papio spp.) groups where numerous strong ties existed within matrifocal social 
groups, but intergroup connections were rare. Many of the themes of Maryanski's work — such as a focus on emergent population structure and its implications for patterns of flow over the network — are shared by modern SNA. Her approaches were largely descriptive, however, and lacked many of the formal, quantitative elements embraced by network analysis today. Indeed, the recent surge of interest in SNA has in part been driven by the availability of modern computing power, as well as programs specifically designed for SNA (e.g., Borgatti et al., 2002; Whitehead, 2009), thereby allowing researchers to go beyond simple network metrics—-such as degree and strength—and to apply a more rigorous statistical framework to their data (Croft et al., 2008; Brent et al., 2011).

A landmark paper that opened the floodgates for applying SNA to nonprimate species was David Lusseau's application of these techniques to a bottlenose dolphin (Tursiops spp.) population in Doubtful Sound, New Zealand. Lusseau (2003) analyzed a population of 64 dolphins and found a social network characterized by a high level of clustering-i.e., the network was fairly cliquish in that an individual's network neighbors were also likely to be associated with one another (Box 1). Despite these strongly associated subgroups, simulation studies in which randomly selected individuals and all their connections were removed from the network demonstrated the network's resilience to perturbation. Numerous pathways connected each dolphin to others in the population such that random removal of individuals did not fragment the larger network. Artificially constructed random networks with the same number of nodes and links as the real network fragmented much faster into isolated subgroups when experiencing the same level of random node removal. Targeted, nonrandom removal of especially highly 
connected individuals had a greater effect on overall social structure, but still did not fragment the network. Additional work on this population has demonstrated the existence of smaller communities within the larger network, as well as the presence of particular individuals connecting these communities together who could potentially have a disproportionate influence on transmission processes occurring over the network (Lusseau \& Newman, 2004; Lusseau et al., 2008).

Lusseau's (2003) study was a major step forward in animal SNA. Rather than just describing the structure of the network, he utilized methods that allowed him to make wider inferences regarding both its structure and function. For example, the structure of the dolphin network was compared to that of random networks of equivalent size and density to emphasize potentially important aspects of dolphin social organization. Further, in testing whether particular pairs of dolphins were found together significantly more often than expected by chance, the permutation methods used to generate randomized networks maintained the underlying data structure-i.e., the observed group sizes and the number of times each individual was observed. These methods provided a more realistic null model for the observed data as compared to the node-label permutation methods more commonly used by sociologists (James et al., 2009). In addition, Lusseau focused on the emergent properties that could arise from network structure, such as facilitating rapid flow of social information and providing resilience in terms of network structure and function to the loss of population members.

Croft et al. (2004) applied SNA to a wild Trinidadian guppy (Poecilia reticulata) population in the Northern Range Mountains of Trinidad. All adult guppies were collected from a pool within the Arima River, individually marked with colored 
elastomer, and released back into the same pool. Shoals were then recaptured over a 7day period to construct the social network. Croft et al. (2004) demonstrated that though guppies exhibit a highly dynamic, fission-fusion social system in which shoal membership can change rapidly as shoals meet with and diverge from each other, this population still possessed a highly structured social network. In particular, the network had a short mean path length and a high mean clustering coefficient, suggesting information and disease could spread rapidly throughout the population. The path length between two individuals is the smallest number of edges that lie between them on the network - e.g., a direct connection means a path length of 1 -while the clustering coefficient measures the extent to which an individual's associates are themselves linked (Box 1). Persistent associations were present in this population between pairs of females even after accounting for body-size preferences, suggesting shoaling decisions could be based on active partner choice. These associations persisted over several days, despite the fact that guppy shoals disperse at night and reform anew each morning (Croft et al., 2003). While a preference for familiar individuals had been demonstrated in guppies under laboratory conditions using binary choice trials (Griffiths \& Magurran, 1997), Croft et al. (2004) provided compelling evidence that such preferences can also be expressed in wild populations.

Over the past decade, SNA has become an increasingly popular technique to probe aspects of social structure and to study behavior within the larger social context in which animals are embedded (Croft et al., 2008; Whitehead, 2008; Sih et al., 2009; Pinter-Wollman et al., 2014). It has been used in a number of taxa and applied to topics spanning the range of behavioral ecology (Wey et al., 2008; Sih et al., 2009). For 
example, SNA has allowed for detailed descriptions of social structure to be made for numerous species, including ants (e.g., Odontomachus hastatus) (Jeanson, 2012), blacktip reef sharks (Carcharhinus melanopterus) (Mourier et al., 2012), and reticulated giraffes (Giraffa camelopardalis) (VanderWaal, Wang, et al., 2014). Other studies have used SNA to examine the links between social structure and behavior, exploring topics that include song development in brown-headed cowbirds (Molothrus ater) (Miller et al., 2008) and how behavioral type influences the composition and structure of cooperative breeding groups in a cichlid species (Neolamprologus pulcher) (Schürch et al., 2010).

SNA has also been used to explore how social structure influences populationlevel processes. Transmission pathways for the transfer of food items, and their implications for the spread of pathogens, have been explored in honeybee hives (Apis spp.) (Naug, 2008), while the structure of networks based on refuge sharing were found to be important predictors of parasite load and infection probability in both gidgee skinks (Egernia stokesii) and sleepy lizards (Tiliqua rugosa) (Godfrey et al., 2009; Leu et al., 2010). Taking advantage of a 10-year data set on long-tailed manakins (Chiroxiphia linearis), David McDonald has employed SNA to address topics such as the importance of early life social connections in predicting later social rise and reproductive success (McDonald, 2007), and the extent to which kin selection has played a role in the evolution of obligate cooperative relationships in this species (McDonald, 2009).

SNA allows us to address several of the aspects of sociality laid out by Wilson (1975) in an integrated, quantitative framework that can facilitate objective comparison between individuals, populations, and species (Faust \& Skvoretz, 2002; Kelley et al., 2011; Wilson et al., 2013). Even more exciting, as other fields utilizing network analysis 
develop in parallel with behavioral ecology, cross-pollination of concepts and techniques have occurred and will continue to, thereby enriching our field with an influx of new hypotheses and methods to test them. While network analysis in behavioral ecology has been largely descriptive up until now, that too is beginning to change as experimental manipulation, new technologies, advanced statistical techniques, and simulation modeling allow researchers to transition into an explicitly predictive and explanatory framework (Pinter-Wollman et al., 2014). We will touch on several of these new developments and approaches throughout the remainder of the review as we turn now to recent work that has been done using SNA and explore how a network-based approach has given us new insights into problems both old and new.

\section{Social Network Analysis and Topics in Behavioral Ecology}

Sih et al. (2009) listed four concepts embraced by SNA that are of particular importance to behavioral ecology: (i) individuals differ in their social experiences, (ii) indirect connections can be as important as direct ones, (iii) individuals differ in the extent of their influence within the social network, and (iv) the social network structure in one context can carry over to influence the network structure in other contexts. These four concepts offer a useful framework through which to appreciate the contributions SNA has made to our understanding of various topics in behavioral ecology, including: dominance hierarchies (Shizuka \& McDonald, 2012; Dey et al., 2013), sexual selection (Oh \& Badyaev, 2010), disease ecology (Cross et al., 2004; Godfrey et al., 2009; VanderWaal, Atwill, et al., 2014), and the influence of social structure on fitness (Royle et al., 2012; Wey \& Blumstein, 2012). Here, we have chosen to focus on SNA as it relates to: (i) social learning and information diffusion, (ii) collective movement and 
decision-making, (iii) animal personalities, and (iv) cooperation. These topics were selected because we believe they provide excellent examples of how our understanding of social behavior can be enhanced by explicitly incorporating information on social structure and dynamics into models and analyses of animal behavior.

\subsection{Social Learning and Culture}

The ability of animals to learn environmentally relevant information and novel behavior patterns through observation of other individuals — or the by-products of their behavior-is termed social learning (Hoppitt \& Laland, 2013). Classic examples include social learning of potato washing in Japanese macaques (Macaca fuscata) (Kawai, 1965), milk-bottle opening in great tits (Parus major) (Fisher \& Hinde, 1949), and transfer of novel food preferences through breath odor in Norway rats (Rattus norvegicus) (Galef et al., 1984).

Early theoretical models exploring the adaptive value of social learning assumed a well-mixed population in which social learners copied others in a more or less random fashion (Boyd \& Richerson, 1985; Rogers, 1988). The results of these models suggested that social learning did not automatically increase individual fitness as was generally assumed. Rather, negative frequency dependence for social learning resulted in a polymorphic equilibrium where the average fitness of social and asocial learners was equivalent (Rogers, 1988). This finding, known as Rogers' "paradox," flew in the face of common assumptions that the ability to use social learning must enhance fitness, using the success and growth of human populations as evidence. One way out of the "paradox" was by recognizing that social learning usually does not occur as random copying of others within a homogeneous population. Rather, humans and other animals are more 
likely to acquire social information in highly selective ways that are influenced by demonstrator characteristics, as well as by differential access to those demonstrators (Coussi-Korbel \& Fragaszy, 1995; Laland, 2004; Hoppitt \& Laland, 2013). In short, when exploring social learning dynamics, the social network structure matters.

\subsubsection{Theoretical Models of Social Learning and Culture in Structured Populations}

Recent theoretical studies have begun incorporating elements of social structure and learning strategies into their models to better explore how cultural traits spread through and persist in a population, the conditions that influence cultural trait diversity, and the fitness consequences of these traits. Given the obvious importance of culture in our societies, most work on these topics has focused on humans. From this body of research, it is clear that social structure can greatly influence how quickly, and to what extent, cultural information transmits through a population. For example, the mean network degree-i.e., the average number of connections an individual has - predicts the ultimate spread of a cultural trait, while the extent to which a network is formed of smaller, semi-isolated subgroups influences how quickly an equilibrium state is reached through the loss of neutral cultural variants (Holme \& Newman, 2006; Li \& Hui, 2008; Meyers et al., 2005; Nardini et al., 2008).

Fewer researchers have modeled cultural dynamics in nonhuman systems (Whitehead \& Lusseau, 2012). Here, we will use the definition of culture as socially learned behavior that is shared by members of a group (Laland \& Galef, 2009; Whitehead \& Lusseau, 2012), though other definitions with more stringent requirements have also been used in the literature (Laland \& Galef, 2009). Possible examples of nonhuman culture include nut cracking in chimpanzees (P. troglodytes) (Boesch et al., 1994), potato 
washing in Japanese macaques (M. fuscata) (Kawai, 1965), and tool use in New Caledonian crows (Corvus moneduloides) (Hunt \& Gray, 2003) and bottlenose dolphins (Tursiops spp.) (Krützen et al., 2005). By simulating transmission over small social networks with varied structural properties, researchers can gain insight into how putative cultural traits might spread through animal societies.

Voelkl and Noë (2008) constructed artificial networks of varying resemblance to commonly observed natural social structures, as well as simulating the real social network published in Sade's (1972) study of rhesus macaques (M. mulatta). When they examined transmission patterns over these networks, they found transmission rates were highest in a homogeneous network where every individual was equally connected to every other. A chain network where each individual had at most two connections possessed the slowest transmission rates. These highly artificial networks are extreme versions of egalitarian and hierarchical social structures respectively. Between these two extremes, networks with greater resemblance to real social networks observed for nonhuman primates had intermediate transmission rates. The decrease in transmission rate as networks became more hierarchical is consistent with empirical evidence that suggests that dominance relationships can impede the spread of social traits (e.g., CoussiKorbel \& Fragaszy, 1995; Huffman, 1996; Kendal et al., 2010).

Voelkl and Noë (2010) simulated information propagation in over 70 nonhuman primate sociopositive social networks—-for example, networks based on grooming or social tolerance - previously published in the literature. In addition to using the unaltered social network, each network was also modified by: (i) shuffling the weighted edges between nodes to randomize the connection pattern while maintaining the original 

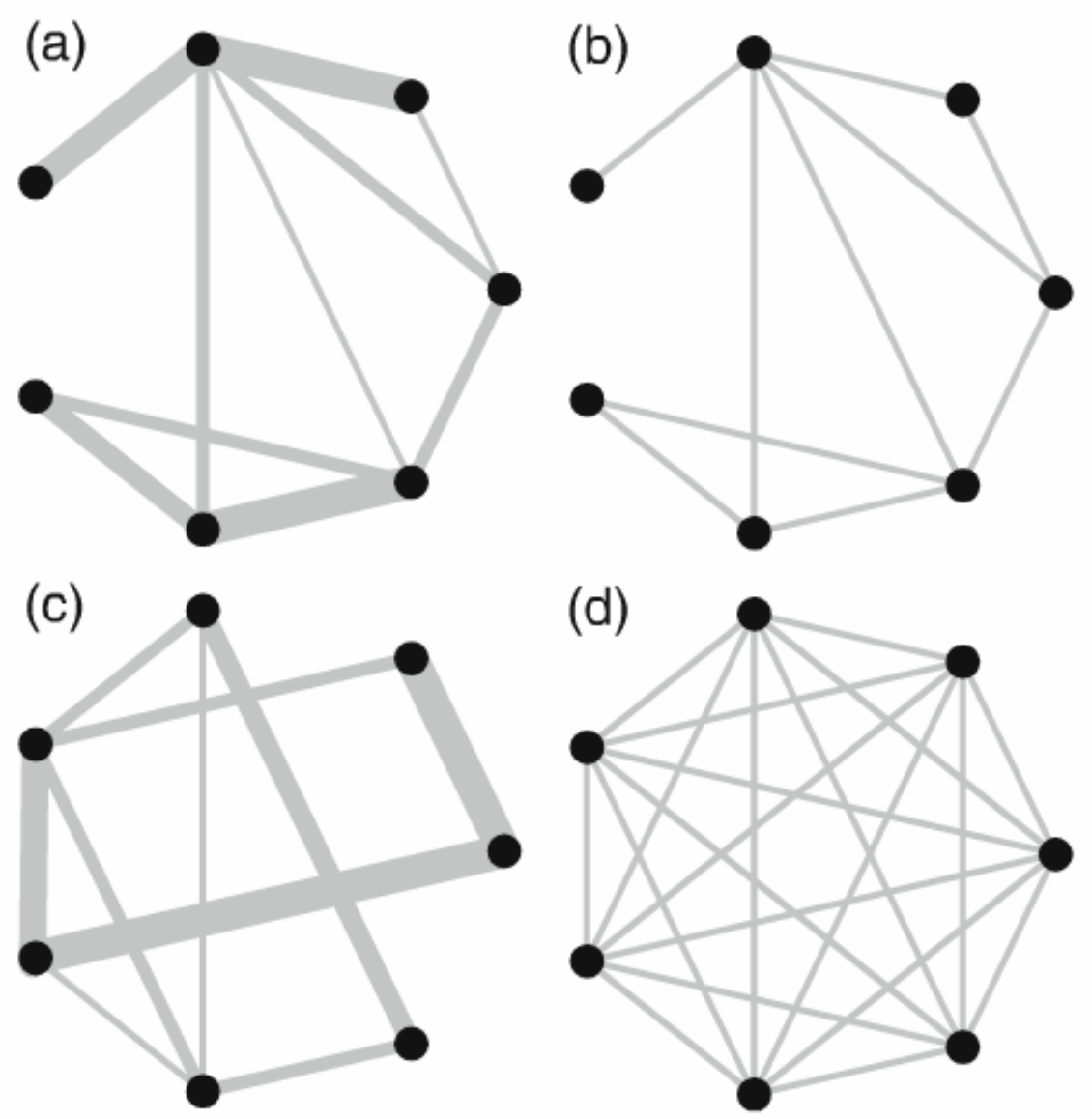

Figure 1.3 The four network types used in Voelkl and Noë's (2010) simulation study of social transmission in primate networks. (a) A network based on an actual primate social network where edge weights represent interaction frequency, (b) a network which maintains the connection pattern of the original network, but removes weighting so that all connections are of equal strength, (c) a network which maintains the distribution of edge weights from the original network, but the connections between nodes are randomized, and (d) a complete network where all possible connections are present and of equal weight. Reprinted with permission from: Voelkl and Nö̈ (2010). Simulation of information propagation in real-life primate networks: longevity, fecundity, fidelity. Behavioral Ecology and Sociobiology, 64, 1449-1459. Copyright @ 2010 Springer Science + Business Media . 
network's distribution of edge weights, (ii) creating an unweighted, binary network that maintained the connection pattern, and (iii) creating a well-mixed, homogeneous network where each individual was connected to every other individual and all network ties were of equal strength (Figure 1.3). Information spread fastest in the well-mixed networks and slowest in the unaltered, original networks. This suggests that social transmission rates were hindered by both the pattern of social connections, as well as the unequal distribution of connection strength.

Whitehead and Lusseau (2012) recently simulated a number of social learning rules within networks of varying structure to explore the relative influence of both factors on cultural diversity. As networks became more modular, cultural diversity increased. Modularity was calculated as the difference between the proportion of total edge weights that connect individuals within the same subgroup and the expected proportion when assuming individuals associate at random (Newman, 2004). This score ranges from 0 for undifferentiated populations without clustering to 1 for a highly differentiated population where individuals only interact within, rather than between, subgroups. Within highly modular populations, clusters of individuals that were socially isolated from the larger population tended to embark on independent behavioral trajectories. This pattern was found regardless of the learning rule being used. Social structure might impose an upper limit on the overall behavioral diversity a network can realize, regardless of the learning mechanisms at work. The authors suggest that these predictions could be tested by comparing behavioral diversity and social structure in wild populations thought to exhibit cultural traditions, such as primates, cetaceans, and songbirds. Taken together, the above studies suggest that as a population becomes more structured-that is, less 
homogeneous — and/or access to other individuals becomes more limited, transmission occurs at a slower rate through the population with the added effect of possibly increasing the overall diversity of whatever it is that is being transmitted-e.g., novel behaviors, diseases.

The above simulation studies allow for generation of predictions which can then be tested in actual animal populations by quantifying the population's social structure, introducing a novel trait, and observing its spread (Voelk1 \& Noë, 2008; Whitehead \& Lusseau, 2012). Potentially valuable new methods to accomplish these tasks have begun to be developed (e.g., network-based diffusion analysis (NBDA); see below). An area for future inquiry is to study these processes over dynamic networks in which connection patterns and strengths can shift over time (Whitehead \& Lusseau, 2012). For example, if connections between parents and offspring begin strong in life and weaken as the offspring ages, vertical transmission rates should be highest early in an individual's life.

\subsubsection{Network-Based Diffusion Methods}

An early approach used to study social learning in freely interacting animal groups was diffusion curve analysis, in which the cumulative number of individuals that possess a trait over time is plotted. Traditionally, the shape of the diffusion curve was used as an indicator of asocial or social learning (e.g., Lefebvre, 1995). An r-shaped curve was taken as evidence of asocial learning as, at the population level, it is indicative of individuals learning a trait at a roughly constant rate. Social learning was inferred from an s-shaped curve. The s-shape suggests few individuals possess the trait early on, resulting in a dearth of demonstrators and slow overall trait acquisition. As more individuals acquire the trait, more demonstrators are available to provide additional 
opportunities for naïve individuals to learn; under these conditions, rapid spread of the trait is predicted. Eventually, there are few uninformed individuals left and the overall rate of acquisition slows.

Using traditional diffusion curve analysis to understand social learning is problematic. These analyses assume all members of a population are equally likely to transmit or receive information and that the population is structured homogeneously with all individuals equally likely to interact with one another (Reader, 2000). Additionally, the shape of the curve is not as indicative of underlying learning processes as was initially assumed. Social learning may not result in a simple, s-shaped curve if populations exhibit some level of substructuring where the trait spreads quickly within, but slowly between, subgroups (Laland \& Kendal, 2003; Reader, 2004). Similarly, if information is more likely to be learned from some individuals than others- e.g., due to transmission biases or directed social learning - the shape of the curve changes. Furthermore, an s-shaped curve can arise from asocial phenomena such as: neophobia, multistep tasks, or if variation in asocial learning rates exists between individuals (Reader, 2004; Hoppitt, Kandler, et al., 2010).

To address these concerns, a new form of diffusion analysis has recently been developed. NBDA infers social learning if the spread of a trait through a population appears to follow the social network: that is, that social information is more likely to spread quickly between animals tightly linked in the network (Coussi-Korbel \& Fragaszy, 1995; Franz \& Nunn, 2009; Hoppitt \& Laland, 2013). NBDA can be based on either the order in which individuals acquired the trait or the actual times of acquisition. These models can be used to compare strength of social transmission between contexts (e.g., 
open vs complex environments: Webster et al., 2013) or to test hypotheses related to different social learning strategies thought to be at work in a population (e.g., copying familiar individuals: Atton et al., 2014; vertical transmission: Allen et al., 2013). Factors predicted to influence asocial rates of acquisition, such as neophobia or boldness, can also be incorporated into the models to control for their effects (Hoppitt, Boogert, et al., 2010).

While still a new technique, NBDA has already been employed in both laboratory and field studies on a number of species, including: three-spined sticklebacks (Gasterosteus aculeatus) (Atton et al., 2012; Webster et al., 2013; Atton et al., 2014), multiple species of Paridae songbirds (Aplin et al., 2012; Aplin et al., 2015), ring-tailed lemurs (Lemur catta) (Kendal et al., 2010), red-fronted lemurs (Eulemur rufifrons) (Schnoell \& Fichtel, 2012), and humpback whales (Megaptera novaeangliae) (Allen et al., 2013). So far NBDA has been used primarily to study the spread of foraging information related to locating and accessing food, but it has the potential to address nearly any behaviorally transmitted trait-e.g., vocal traditions in cetaceans (Noad et al., 2000), mate-choice copying (Dugatkin, 1992), or defensive behaviors (Magurran \& Higham, 1988; Mineka \& Cook, 1988).

NBDA offers several important advantages for studying social transmission. Many social learning studies place individuals in binary choice tests following observation of informed demonstrators. While such studies have been useful in establishing the mechanisms and behavioral strategies by which individuals acquire and use social information (Galef, 2009; Hoppitt \& Laland, 2013), animals are often tested under highly artificial conditions and restricted from acting within normal social contexts. 
NBDA's primary benefit is that it allows researchers to study social learning in naturalistic contexts with freely interacting groups of animals. Additionally, ecological, genetic, and social factors thought to influence the spread of a behavior can be considered simultaneously through inclusion of the appropriate variables in the NBDA model. This provides an attractive alternative to the ethnographic method which instead attempts to infer social learning in the wild through ruling out alternative genetic and ecological explanations (Laland \& Janik, 2006). For example, lobtail feeding in humpback whales might be a behavioral specialization for foraging on a particular prey species: sand lance (Ammodytes americanus); the initial occurrence of this behavior in the humpback population in the Gulf of Maine coincided with both an increase in sand lance abundance and a dramatic decrease in the abundance of another important prey species for humpbacks: herring (Clupea harengus). Allen et al. (2013) found support using NBDA for both social transmission of lobtail feeding and for ecological effects-i.e., annual sand lance biomass—influencing acquisition of the lobtail technique.

One exciting possibility offered by NBDA that has only begun to be explored is examining the use of social learning strategies under various social and ecological conditions in freely interacting groups. For example, Atton et al. (2014) found that familiarity between two sticklebacks (G. aculeatus) facilitated discovery of a novel food source. The pattern of information acquisition in shoals made up of both familiar and unfamiliar fish was best described by a network allowing information flow only between familiar individuals; these results are largely consistent with a social learning strategy of “copy familiar individuals" (Galef, 2009). 
Aplin et al. (2015) found strong evidence for a conformist strategy (i.e., "copy the majority") influencing the establishment and persistence of alternative, but functionally equivalent, novel behaviors in wild networks of great tits (P. major). Demonstrators from each subpopulation were trained in captivity to open a puzzle box with one of two options - pushing the blue half of a sliding door to the right or the red half to the left — in order to access the mealworms contained within. Demonstrators were then released back into their original subpopulations. Puzzle boxes with both options available were provided for each subpopulation and the timing and pattern of problem-solving were monitored. NBDA found overwhelming support for social transmission of these behaviors compared to asocial learning; birds with strong connections to solvers were much more likely to solve the task themselves. Despite both options — red and blue doors - being available for use, birds usually first solved the task with the option originally seeded into their subpopulation and continued to strongly prefer this option even after learning of the alternative. When the puzzle feeders were returned to the woods after a 9-month absence, each subpopulation still preferred their initially seeded technique despite significant population turnover. A cultural tradition-that is, a socially learned behavior shared within a group — appears to have been established in these populations, transmitted via the social network, and maintained through conformist biases for at least two generations.

Finally, an important feature of NBDA is that estimates of the strength of social transmission can be obtained. This is in contrast to earlier network-based diffusion methods that simply compared a test statistic to a null distribution generated with randomization techniques (e.g., Boogert et al., 2008; Morrell et al., 2008). For example, 
one of the approaches used in Boogert et al. (2008) used a weighted social network and the order in which individuals acquired a behavior (i.e., the diffusion chain). The average association strength between each individual and all those who preceded it in the diffusion chain was summed over all individuals in the chain. This test statistic was then compared to a distribution of null values generated using randomized diffusion chains. If the trait was socially transmitted through strong network connections, the test statistic was expected to be in the upper $2.5 \%$ of the distribution. However, this method only indicates whether or not social transmission was likely occurring; it provides no estimates regarding the strength of social transmission. The effect size estimates possible using the latest forms of NBDA can facilitate comparisons of the strength of social learning between different contexts, populations, and species to better identify conditions that promote or may have selected for enhanced social transmission (e.g., Webster et al., 2013). It might even be possible to predict future spread of information through a population if given sufficient information on the effects of relevant factors on social transmission rates. This could have ramifications for management of wild and captive populations, such as seeding beneficial information or training to the individuals most likely to facilitate its rapid spread (Makagon et al., 2012).

Despite its potential utility, NBDA has weaknesses that require careful consideration from researchers prior to applying it. While NBDA facilitates studies of social learning under wild conditions, the ability for researchers to accurately identify when an individual has acquired the trait of interest is critical. Observation errors regarding this information can decrease the power of NBDA to detect social learning (Franz \& Nunn, 2010). Analyses based only on the order of acquisition can be potentially 
more resistant to these errors, but may also have less statistical power to detect social learning than analyses that also incorporate information on the time of acquisition (Franz \& Nunn, 2010; Hoppitt, Boogert, et al., 2010). Identifying an appropriate association measure is also critical. Ideally, a measure should be selected that reflects the probability that information will transmit between two individuals-e.g., a network based on how often individuals feed together might be more appropriate when considering the spread of foraging-related traits than a network based solely on spatial proximity (Hoppitt, Boogert, et al., 2010; Hoppitt \& Laland, 2013).

On a more conceptual note, NBDA uses a static network constructed from aggregated observations of association or interaction. If transmission processes occur at a fast rate relative to changes in network structure, then NBDA is a powerful technique. If instead transmission processes and structural changes occur over similar timescales, then a more fine-grained view of social dynamics might be needed (Croft et al., 2008; Wilson et al., 2014). Furthermore, if acquiring a trait changes an individual's interaction patterns, then a social network constructed prior to when an individual acquired a trait might not accurately reflect its social relationships after trait acquisition. A model allowing for a dynamic network that can change over time might be more appropriate in this context (e.g., Blonder et al., 2012). New approaches are being developed that could help address some of these shortcomings; we will discuss a few of them-e.g., Markov chain models, dynamic networks-later in the review.

In addition to diffusion studies per se, attempts have been made to link particular network metrics with social learning. For example, high eigenvector centrality (Box 1) suggests an individual is well connected in its network and might therefore experience 
increased access to beneficial social information, resources, or mating opportunities. Formally, eigenvector centralities are taken from the first eigenvector of the matrix of associations or edges (Newman, 2004). In practical terms, an individual can have high eigenvector centrality if it has many connections in the network-i.e., high degree or strength—or if it is connected to individuals who have many connections. In squirrel monkeys (Saimiri sciureus), an individual's eigenvector centrality predicted the likelihood of, and the speed with which, it solved a foraging-related task when trained demonstrators were introduced into the group (Claidière et al., 2013).

Information centrality measures the extent to which one individual links pairs of other individuals together, thereby providing an indicator of how important an individual is in influencing the flow of information through its network (Stephenson \& Zelen, 1989). It is similar to the betweenness of a node, but betweenness only counts the number of shortest paths between pairs of nodes that pass through the node of interest (Freeman, 1979). In comparison, information centrality takes into account all possible pathways weighted by the inverse of their length. Vital and Martins (2011) found that individuals who were characterized by high information centrality were of greater importance to group function than noncentral individuals in zebrafish (Danio rerio) shoals. Removal of these central fish disrupted the ability of the group to learn foraging-related cues, while removal of other fish had little effect (Vital \& Martins, 2011). An intriguing direction for future research is suggested by the strain-related differences found in Vital and Martins' study which hint at underlying genetic bases to both zebrafish social organization and learning. 


\subsubsection{Transmission Dynamics Using Markov Chain Models}

A recent development in the analysis of animal networks with important implications for understanding transmission processes is the use of Markov chain models which allow for exploration of the social dynamics that drive the formation of a network (Wilson et al., 2014). Wilson et al. (2014) conducted repeated focal follows of wild female guppies $(P$. reticulata) in their native habitat of Trinidad, periodically recording whether or not the focal fish was shoaling with another female and if so, with who. From these observations, they constructed Markov chain models describing shoaling dynamics, where the shoaling behavior of an individual at time $t+1$ depends solely on its behavioral state at time $t$, and each behavioral state-e.g., shoaling, swimming alone - is associated with a unique set of transition probabilities describing the likelihood of future states. Simulated outputs from a number of Markov chain models were compared to the observed data to assess goodness-of-fit (Figure 1.4). Wilson et al. (2014) found that the best-fitting model had focal individuals selecting shoaling partners with individualspecific probabilities, suggesting active social preferences were at work in this population. These Markov chain models were then used to generate simulated networks whose structure was compared to that of real networks constructed from the observed shoaling partner data. These comparisons found that when models without individualspecific shoaling preferences were used to generate simulated networks, the structure of these networks differed significantly from that of the real networks. This difference was not found when models that included individual-specific shoaling preferences were used instead to generate the simulated networks, suggesting these preferences played an important role in determining population structure. 


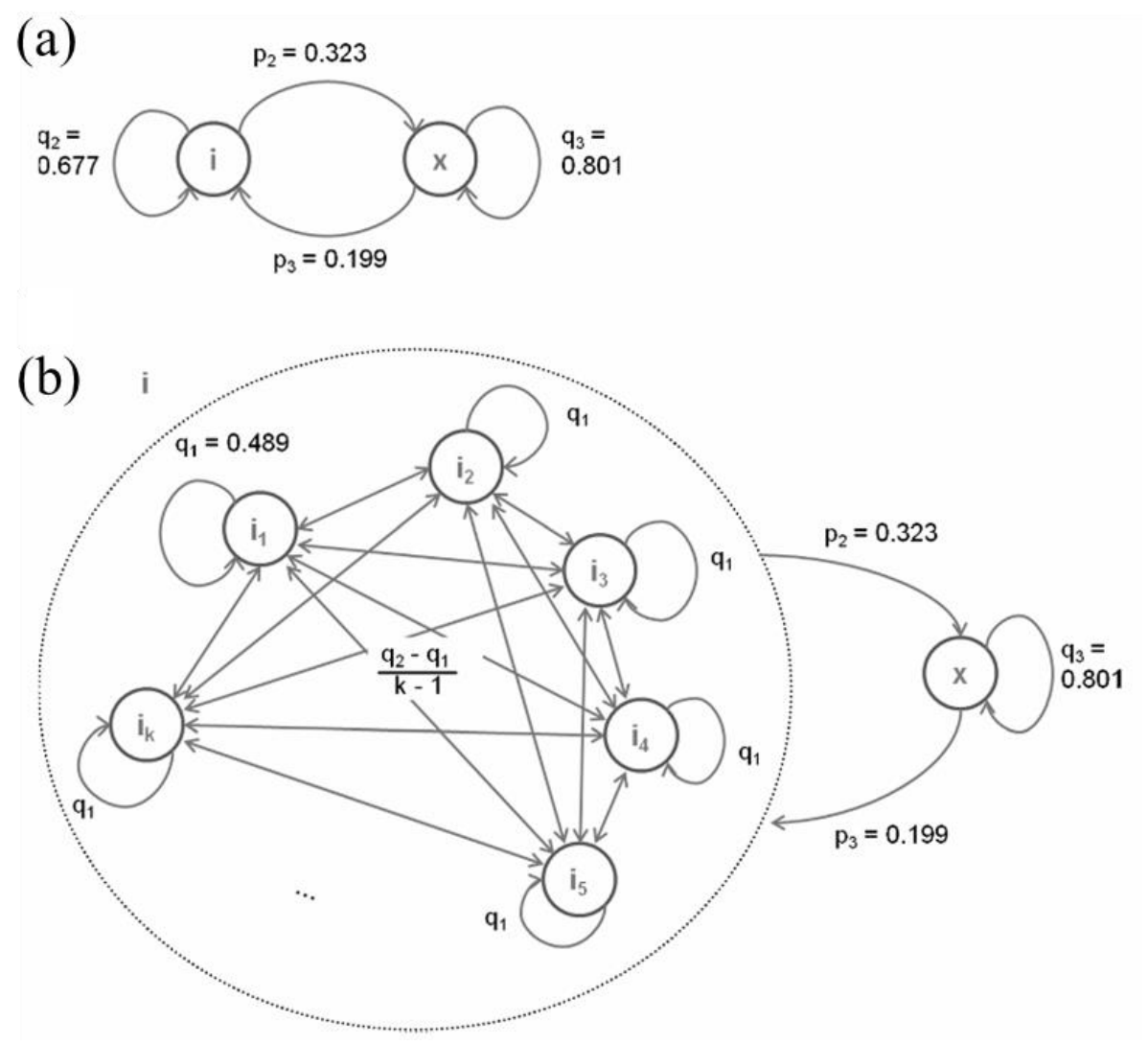

Figure 1.4 Markov chain models of Trinidadian guppy (Poecilia reticulata) shoaling dynamics. (a) The simplest possible model where an individual can either be shoaling $(i)$ or alone $(x)$. The probabilities of switching state are given by $p_{2}$ and $p_{3}$, while the probabilities of maintaining the same state are given by $q_{2}$ and $q_{3}$. (b) An elaboration of the simplest model, in which an additional term is included describing shoaling state $i$ in the presence of $k$ possible partners. Individuals are selected as shoaling partners with equal probabilities, and the focal individual remains with the current shoaling partner with probability $q_{1}$. An elaboration of this model which incorporates individual-specific shoaling preferences provided the best fit to the empirical data (see text). Reprinted with permission from: Wilson et al. (2014). Dynamic social networks in guppies (Poecilia reticulata). Behavioral Ecology and Sociobiology, 68, 915-925. Copyright (C) 2014 Springer Science + Business Media. 
Wilson et al.'s (2014) Markov chain models can be used to analyze and predict transmission processes over networks with a potentially high level of accuracy. These models were used in a disease transmission simulation where it was found that individual partner preferences slowed down infection rates relative to a model assuming a homogeneous social structure with no partner preferences. For this approach to work, some knowledge is needed regarding the length of time required for transmission to occur between two individuals. Animals can then be observed at an appropriate frequency to capture social dynamics at a fine enough scale to properly model the transmission process of interest (e.g., information, disease, parasites). By constructing Markov chain models that explicitly incorporate factors predicted to influence social learning dynamics, such as age, familiarity, or kinship, predictions could potentially be made regarding the importance of various social learning strategies within a population (Laland, 2004; Hoppitt \& Laland, 2013). This approach could also facilitate comparisons of social learning between populations and species.

The use of Markov chains in animal social network studies is still relatively new and its effectiveness has not yet been extensively tested. For instance, it remains unclear to what extent population density might influence the transition probabilities of the Markov chains and consequently limit comparative studies. Nevertheless, it offers an intriguing next step for modeling transmission dynamics over networks, as well as exploring the processes that shape social structure. Wilson et al.'s (2014) approach could be especially appropriate for modeling social dynamics and network structure in animal species where associations and interactions are often short-lived. 
While using Markov chains to model network dynamics is relatively unexplored in the behavioral ecology literature, it has a longer history of use in sociology (Wasserman, 1977; Leenders, 1995; Snijders, 2001; Snijders et al., 2010; Pinter-Wollman et al., 2014). For example, stochastic actor-based models can explore how characteristics of actors, dyadic relationships between pairs of actors, and the actors' positions in their network drive changes in network structure over time. These models use a time series of networks, where the networks are constructed for the same group of actors at each time point. Changes in network structure between time points are modeled as a Markov process where future network structure is determined only by the current state of the network, mediated through the behavior of the nodes. The main difference between stochastic actor-based models and the Markov chain models used by Wilson et al. (2014) is that the former is modeling the changes between multiple observed networks over time, while the latter attempts to identify the social dynamics at work in a population and use them to infer the structure of the population's social network.

\subsection{Collective Movement and Decision-making}

In principle, large-scale, complex, and synchronized movement of animal groups — e.g., fish shoals, bird flocks, insect swarms — might be the result of selforganization based on simple behavioral rules played out at the local level between adjacent group members (Sumpter, 2006). Interacting with neighbors based on rules-ofthumb such as "avoid collisions" and "move toward and align with conspecifics" can result in a cohesive and responsive animal group that can potentially acquire information more effectively than a lone individual could and rapidly disseminate that information to its members (Couzin et al., 2002; Ioannou et al., 2011). Minor variation in these local 
(b)

(a)

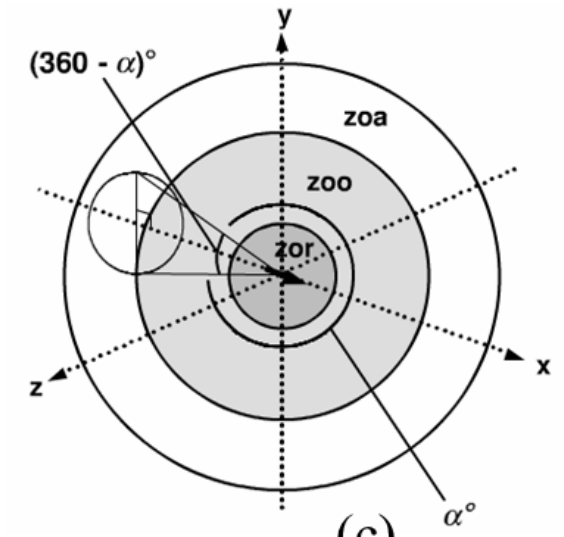

(c)
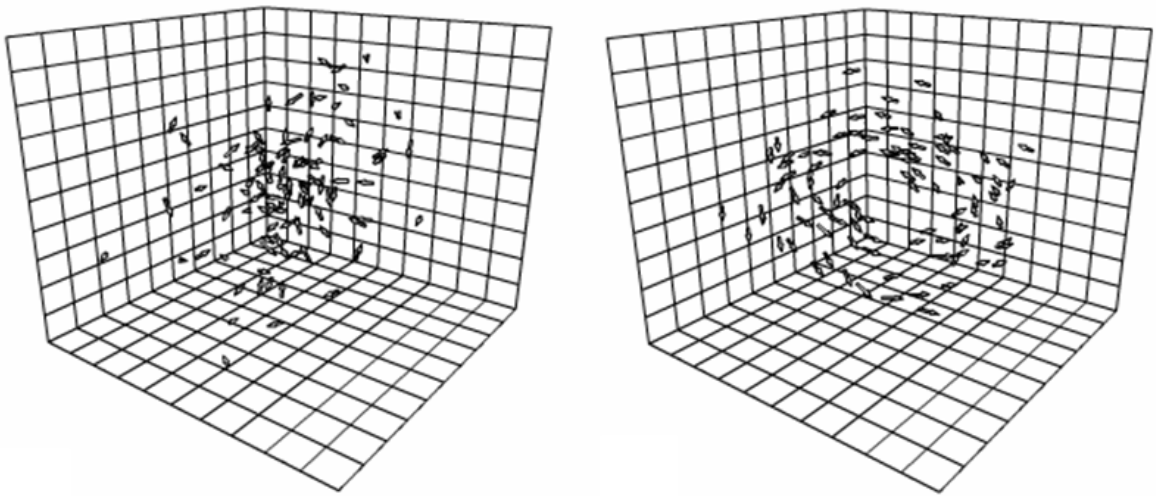

(d)

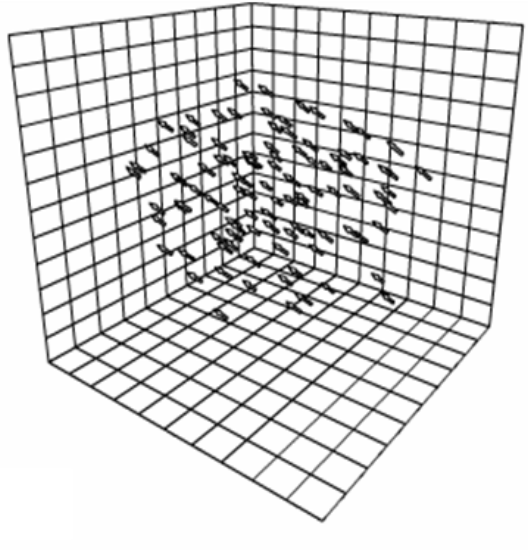

Figure 1.5 A model of collective motion. (a) An individual is centered within three zones governing its local rules. These are: the zone of repulsion (zor), zone of orientation (zoo), and zone of attraction (zoa). An individual's field of perception is determined by $\alpha$. (b) Swarm, (c) torus, and (d) parallel directional formations. Reprinted with permission from: Couzin et al. (2002). Collective memory and spatial sorting in animal groups. Journal of Theoretical Biology, 218, 1-11. Copyright @ 2002 Elsevier. 
rules can lead to rapid and discrete shifts in group structure; for example, simulated fish shoals can shift from swarming behavior to a torus structure and finally to parallel directional movement by simply varying the range over which an individual aligns with group members (Figure 1.5) (Couzin et al., 2002). Each of these formations can be characterized by its influence on group properties, such as cohesion or the speed of information transmission between group members.

Most work on collective movement, however, has not considered how individuallevel rules might be influenced by the underlying social structure of a group. Researchers have simply assumed that the same collective movement rules apply to any conspecifics detected within an individual's perceptual zone (Couzin et al., 2002; Hemelrijk \& Hildenbrandt, 2008). Yet, empirical work has shown that phenotypic assortment and social preferences for particular individuals influence animal grouping patterns (e.g., Griffiths \& Magurran, 1997; Croft et al., 2009; Aplin et al., 2013). Incorporating such preferences - embodied within a group's social network-into models of collective motion and decision-making will help to generate new, testable predictions for the field (Bode et al., 2011a).

Research suggests that subtle behavioral variation is sufficient to consistently influence spatial position in a moving group (Couzin et al., 2002); variation in social preferences is predicted to have a similar effect (Bode et al., 2011b). In addition, if animals are more likely to be consistently found in certain spatial locations within a group, this may facilitate the development of social preferences between adjacent individuals (Bode et al., 2011a). Differences in information status, speed of travel, behavior, and other variables can result in passive assortment (Krause et al., 2000; Reebs, 
2000; Couzin et al., 2002), which might then transform into active preferences expressed within the social network.

A caveat about the relationship between spatiotemporal proximity and animal social networks is in order here. In many species and contexts, gathering a good record of the interactions occurring between individuals can be prohibitively difficult (Whitehead, 2008). Social relationships in these networks are often inferred based on the frequency of association instead. Whether two animals are associating or not is usually based on group membership —i.e., "the gambit of the group" (Whitehead \& Dufault, 1999)—where group is defined as animals maintaining spatiotemporal proximity for primarily social reasons. While spatiotemporal proximity often correlates with the likelihood two animals will engage in some social interaction, animals might also group for nonsocial reasons. For example, animals might aggregate around some resource or environmental feature, such as a watering hole or roosting site (Krause \& Ruxton, 2002). In such circumstances, group membership provides much less, if any, meaningful information about social structure (Whitehead, 2008). In other contexts, animals might group for social reasons, but "the gambit of the group" as it is normally implemented, such as through use of a chain rule, can be misleading. For instance, it seems unlikely that every herring $(C$. harengus) shares a meaningful social relationship within a school of potentially thousands of individuals spread out over hundreds of meters (Mackinson, 1999). Instead, it is more likely that an individual fish would maintain many fewer-if any-persistent, social relationships and is most likely to interact with the individuals immediately around it at any one time-i.e., those within its perceptual zone (Couzin et al., 2002). 
Many of the studies we review in this section distinguish between a network of social preferences and the interactions that occur within an individual's perceptual zone (e.g., Bode et al., 2011a, b). The former quantifies stable preferences that change relatively slowly compared to interactions occurring in the latter, which form and break as individuals move near to and away from one another. Additionally, several of these studies, particularly those dealing with group movement toward some resource, demonstrate how the outcomes of self-organized, collective behavior can be influenced by a combination of both social and nonsocial processes. We examine three ways in which social network structure influences different aspects of collective behavior. The first considers collective motion in animal groups that are not actively navigating toward some goal. The second section then considers group navigation and leadership effectiveness. Finally, we turn to topics related to group decision-making and the initiation of group movement.

\subsubsection{Collective Motion in Nonnavigating Groups}

Simulations that incorporate social preferences which bias individual movement toward or away from particular individuals have found that the spatial arrangement of individuals within a moving group reflect the group's social network structure. Qiu and $\mathrm{Hu}$ (2010) constructed social networks with weighted edges representing the relative influence each individual had over one another's movement decisions. During collective motion, individual movements were biased toward network neighbors that had greater influence-i.e., stronger edge weights. When the social network resembled a linear chain where each individual had a strong connection with only one other individual, moving groups likewise assumed a linear formation (Figure 1.6(a)). Conversely, where one or a 
(a)
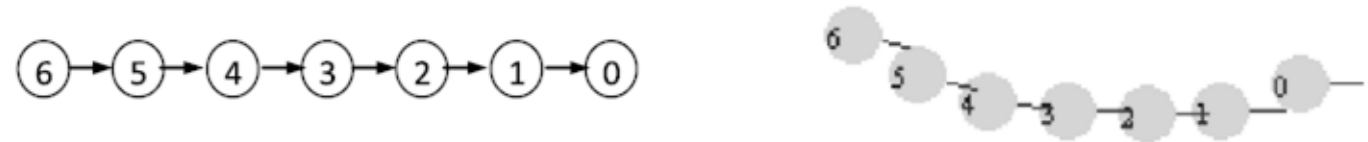

\section{Linear Network Structure and Simulated Movement}

(b)
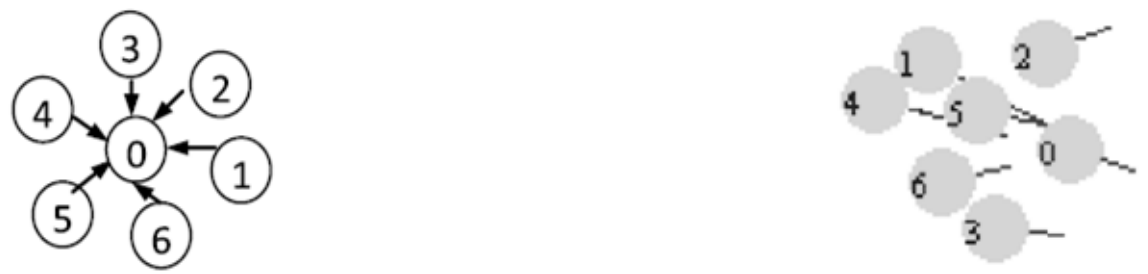

Centralized Network Structure and Simulated Movement

Figure 1.6 Network structure can influence the resulting spatial structure during agentbased simulations of collective motion. (a) A linear network structure in which each individual shares a strong social connection with just one other individual results in a linear formation during collective motion. (b) A highly centralized network structure in which one individual possesses a greater number of strong social connections relative to other group members results in a compact, clustered formation during collective motion. Reprinted with permission from: Qiu and Hu (2010). Modeling group structures in pedestrian crowd simulation. Simulation Modelling Practice and Theory, 18, 190-205. Copyright @ 2010 Elsevier. 
few individuals had particularly high centrality—i.e., they had many strong connections directed toward them — relative to other group members, moving groups formed relatively compact structures as socially peripheral individuals attempted to remain close to these strongly connected, central individuals (Figure 1.6(b)).

Hemelrijk and Kunz (2005) noted similar clustering around preferred associations when they constructed a model incorporating social preferences resembling familiar and unfamiliar associations, with the assumption that familiar individuals preferred one another's company. In their simulations, distinct clusters of familiar individuals were detected within the moving collective. Bode et al. (2011b) explored the influence of various social network structures on collective movement in the absence of navigation. Socially central individuals - i.e., those with numerous strong ties to others in the network-were more likely to also occupy spatially central positions within the moving group. Highly centralized networks that had one or two "key"-i.e., socially central— individuals were found to be tightly cohesive during collective motion. In contrast, strongly interconnected subgroups, such as communities within the network, facilitated fragmentation of the larger group. The most cohesive groups, however, were those that lacked any strong connections, suggesting that a more homogeneous social structure facilitated cohesive collective motion.

\subsubsection{Collective Navigation}

If one or a few individuals possess accurate environmental information, they can lead uninformed group members toward a target, such as a food source, resulting in accurate group navigation (Couzin et al., 2005). Bode, Franks, et al. (2012) asked whether an underlying social network expressing individual preferences influenced the 
ability of leaders to guide group movement. Leaders in their simulations had a preferred direction - that is, they acted as if they were informed regarding the location of a desired destination—while nonleaders had no preferred direction (Figure 1.7(a)). Leaders also had to balance a nonsocial tendency to navigate toward their preferred direction with a social tendency to respond to the movements of group members. At one extreme, leaders only navigated, with no regard for the behavior of group mates. At the other end, no navigation occurred and the leader only moved according to local interaction rules modified by social preferences. Navigational success for the leader increased with its navigational tendency (Figure 1.7(b)). However, if leaders paid too little attention to the behavior of group members (i.e., they focused solely on navigation), group fragmentation could result (Figure 1.7(c)). Fragmentation became less likely when a leader had more and stronger social ties to other group members - that is, when leaders possessed high network centrality. The most effective collective navigation was achieved when leaders had high centrality coupled with moderation in their navigational tendencies (Figure $1.7(d))$.

Although Bode, Franks, et al.'s (2012) models have not been explicitly tested, some empirical results do support their general conclusions. Leadership during flight in homing pigeon (Columba livia) groups can be influenced by individual navigational experience and route fidelity (Freeman et al., 2011; Flack et al., 2012) - that is, those pigeons with higher navigational tendencies were more effective leaders. Vital and Martins (2013) quantified the social network structure of small zebrafish (D. rerio) groups and identified "key" and "nonkey" fish based on individual information centrality (see Box 1). In each group, either a "key" or "nonkey" fish—i.e., those individuals with 


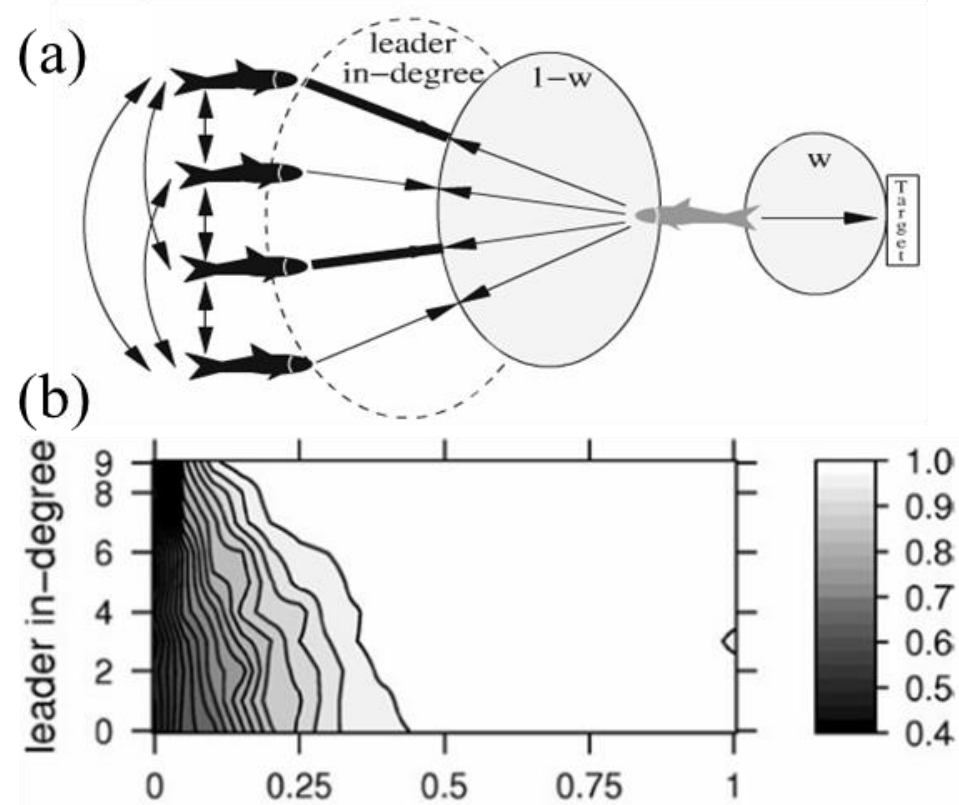

(c)
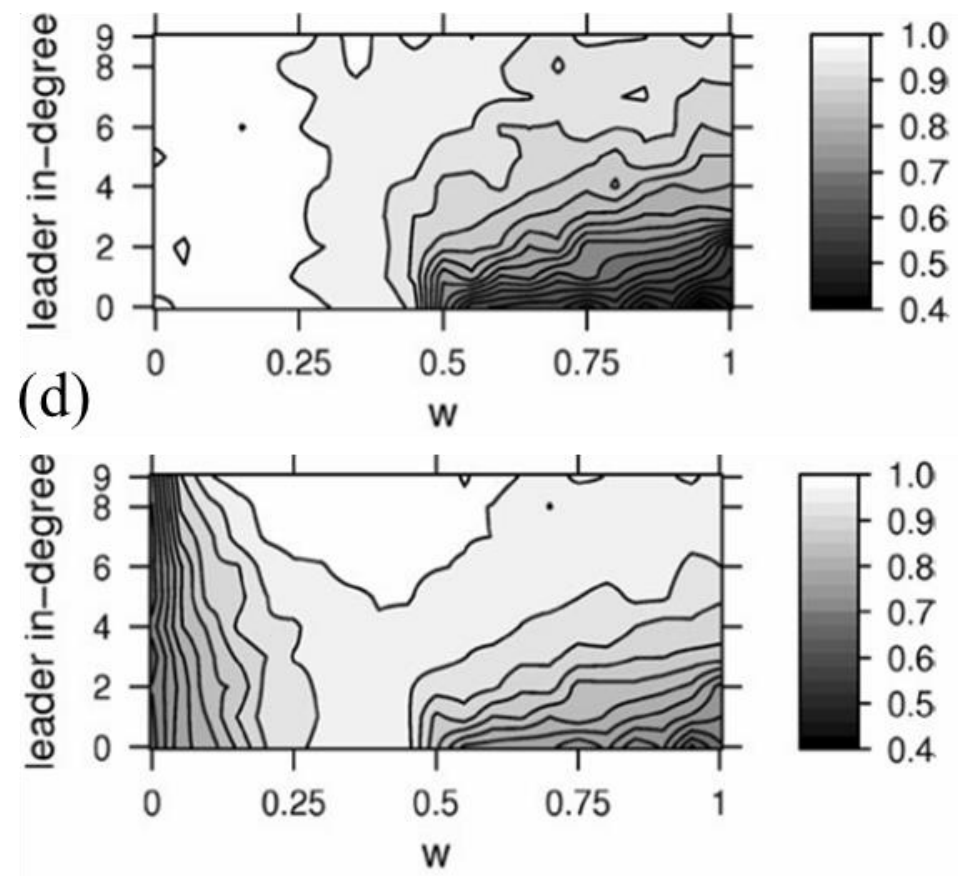
Figure 1.7 (a) The informed individual in gray has a navigational tendency, $w$, countered by social tendencies, $1-w$. Arrows indicate social preferences, with stronger preferences indicated by thicker lines. (b) Navigational success of the leader increased with $w$; as indegree increased, a smaller $w$ was needed for success. (c) The scale on the right denotes the fraction of the total group that was found in the same cohesive group as the leader. More fragmentation was observed as $w$ increased, but this was countered to some extent by higher leader in-degree. (d) The square root of the product of the measures from (b) and (c) provided a combined measure where high values indicated successful group navigation. The most effective group navigation occurred when leaders had high indegree and moderate navigational tendencies. Reprinted with permission from: Bode, Franks, et al. (2012). Leading from the front? Social networks in navigating groups. Behavioral Ecology and Sociobiology, 66, 835-843. Copyright (C) 2012 Springer Science + Business Media. 
the highest or lowest centrality respectively — was trained on how to reach a safe location when faced with an aversive stimulus. When transferred back into their groups, "key" individuals were better able to influence collective group avoidance behavior than “nonkey" fish. Swaney et al. (2001) found that trained guppies (P. reticulata) that were familiar to the rest of the group were better able to lead group mates toward a food source as opposed to trained demonstrators that were unfamiliar to the group. The most effective leaders, though, were the familiar guppies that were relatively poorly trained, as the welltrained fish tended to leave their group mates behind. This seems to parallel Bode, Franks, et al.'s (2012) finding that individuals focused solely on navigation facilitate fragmentation of the larger group, potentially by outpacing other group members and robbing them of effective leadership.

In the absence of informed leaders, animal groups can still accomplish cohesive and accurate navigation. Under what is known as the "many-wrongs principle," pooling imperfect individual navigational information can facilitate collective navigation as individual errors are averaged out at the group level (Simons, 2004). Bode, Wood, et al. (2012) used simulations to examine how social network structure influenced such leaderless group-level navigation. Each individual in the model moved based on both innate, but imperfect, navigational tendencies toward the target direction, as well as social tendencies toward nearby conspecifics. Social preferences were determined by a network of strong and weak connections. Two models of network formation were considered, with the placement of strong connections selected either (i) based on preferential attachment, resulting in a few, very strongly connected nodes (Barabási \& Albert, 1999), or (ii) at random. To represent the absence of social structure, the control 
treatment used a network where all individuals were connected to one another and all connections were equally strong. Bode, Wood, et al. (2012) found that relative to the control treatment, either type of social network acted to reduce group navigation error when individuals were biased in favor of interacting with conspecifics rather than following their own navigational knowledge. Conversely, when individuals focused more on nonsocial navigation, group-level accuracy was no longer influenced by the social network. Overall, these simulations suggest that over an evolutionary timescale, natural selection could favor some level of social structure in group-living organisms due to its facilitation of collective navigation.

The relative scarcity of work combining these two fields can be partially traced to logistical and methodological limitations. It can be difficult enough to track individual wild animals over course timescales, let alone record the fine-grained details needed for studies of collective motion. Novel automated tracking and recording systems are being developed that offer opportunities to begin investigating these topics in greater detail by allowing high-resolution data of individual identities, positions, and movements to be collected for wild animal populations (e.g., Krause et al., 2013; Nagy et al., 2013; Farine et al., 2014; Strandburg-Peshkin et al., 2014).

Researchers have only recently begun to cohesively integrate SNA with studies of collective motion. Given the intriguing results of these initial studies, however, further synthesis of these fields is likely to greatly advance our understanding of the underlying mechanics and dynamics of emergent social behavior. Questions abound: how does the relationship between social structure and information pooling during collective navigation vary across ecological contexts (e.g., within a structurally complex 
environment)? Does social structure mediate or hinder group cohesion when conflicts of interest occur between group members? How do networks characterized both by preferred and avoided relationships influence collective motion and navigation? How are the costs and benefits of leadership balanced against the costs and benefits of maintaining social relationships? Additionally, comparative studies examining the interaction between social networks and collective motion across contexts and between populations and species will allow us to unravel the role natural selection plays in influencing emergent social phenomena.

\subsubsection{Initiation of Group Movement and Group Decision-making}

To this point, we have only considered collective motion in continually moving animal groups without considering how initiation of group movement occurs. Another body of work uses the term collective movement to refer to the sequence of events that include a predeparture period (sometimes with recruitment behaviors involved), initiation of movement, and group movement if the initiator was successful (Petit \& Bon, 2010). In these studies, the focus is on such questions as what individual characteristics are possessed by successful initiators of group movement, and how do animals decide whether to join a departing group or remain where they are?

Joining decisions during the initiation of collective movement have been well studied in primate groups. Jacobs et al. (2011) proposed several rules which brown lemurs (Eulemur fulvus fulvus) might in principle follow in their decision-making process. Joining decisions could be based on: (i) individual-specific needs and motivations, (ii) the identity or characteristics of the initial leader, (iii) the total number of animals who have joined, (iv) the total number of kin who have joined, and (v) the 
affiliative relationships shared with those who have joined. Agent-based simulations of brown lemur behavior found that decisions based on the affiliative relationships shared with those who have joined best fit the data, suggesting that the social network of affiliative behavior predicted group-level movement patterns for brown lemurs. Similar results have been found for Tonkean macaques (Macaca tonkeana) (Sueur et al., 2009, 2010), rhesus macaques (M. mulatta) (albeit modified by kinship patterns: Sueur et al., 2010), and in herds of heifers (Bos taurus) and ewe lambs (Ovis aries) (Ramseyer et al., 2009).

Sueur et al. (2012) further explored how collective movement patterns and group decision-making style were influenced by social network structure by running agentbased simulations using networks varying in their centralization. Highly centralized networks were dominated by one individual with the most and the strongest connections relative to other group members. Specifically, these individuals possessed the highest eigenvector centrality of the group (see Box 1). More decentralized networks were characterized by less disparity in eigenvector centrality between the central individual and its group mates until, in a completely decentralized network, all group members were equally central. In highly centralized networks, the central individual had the greatest recruitment success during initiation of collective movement. As networks became less centralized, leadership effectiveness became more evenly distributed in the group. Finally, in a completely decentralized network, every individual was equally successful as a leader. The interaction between social structure and decision-making style was nonlinear, suggesting that the latter is an emergent property of the former (Bradbury \& Vehrencamp, 2011); once a critical level of network decentralization was reached, group 
decision-making rapidly shifted from an unshared to a shared consensus in which each group member had equal say in collective decisions (Conradt \& Roper, 2005). Results of these simulations were very much in line with the empirical data on movement initiation and joining decisions in a number of primate species, including: Tonkean macaques $(M$. tonkeana), rhesus macaques (M. mulatta), brown lemurs (E. fulvus fulvus), and whitefaced capuchins (Cebus capucinus).

Social network structure can also influence the dynamics of permanent group fissions, with important consequences for patterns of gene flow, population dynamics (e.g., growth rate), colonization of new habitats, and the potential extinction of local or regional populations (Lefebvre et al., 2003; Jones, 2005; Strier et al., 2006). For example, a population of northern muriqui monkeys (Brachyteles hypoxanthus) located in a small forest reserve in Brazil underwent a permanent fission event over the course of 20032005 when a subgroup broke off from the main population (Strier et al., 2006). Tokuda et al. (2014) retroactively employed SNA to examine how social structure might have influenced the dynamics of the fission process. Newman's modularity method (Newman, 2004,2006 ) was used to detect subgroups of individuals within the larger population. Modularity-i.e., the extent to which association occurs within, as opposed to between, subgroups - in the population increased over time as the point of fission approached. Females that were more peripheral to the main group - i.e., those that had relatively lower strength and eigenvector centrality — began to associate as a separate subgroup that eventually broke off from the larger group (Figure 1.8). Ecological factors, such as foraging competition, were implicated in the permanent split in the female population, while the subgroup of males that subsequently joined the new female subgroup likely did 

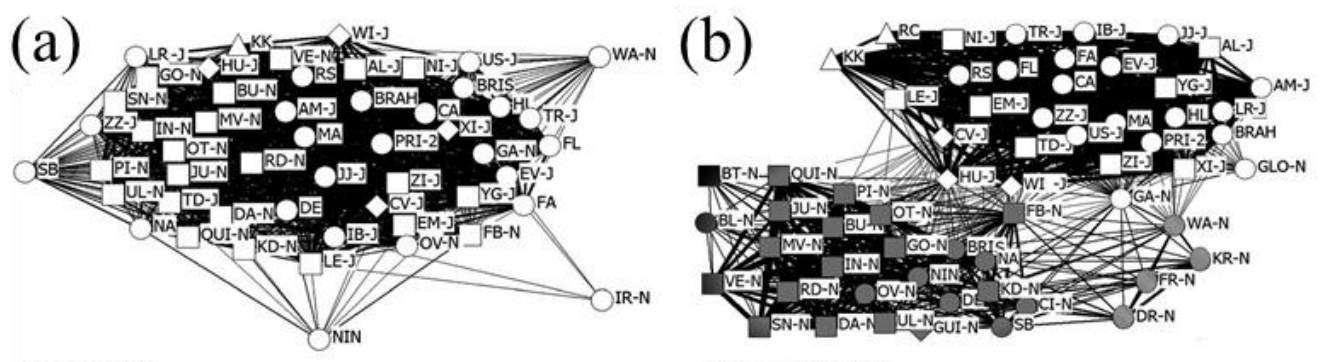

Dry season 2002

Rainy season 2002-2003
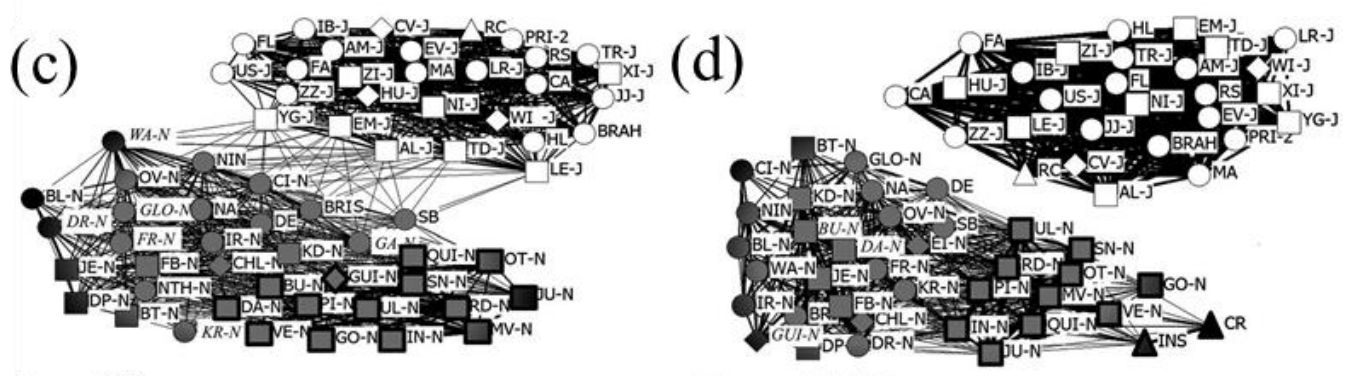

Dry season 2003

Rainy season 2003-2004
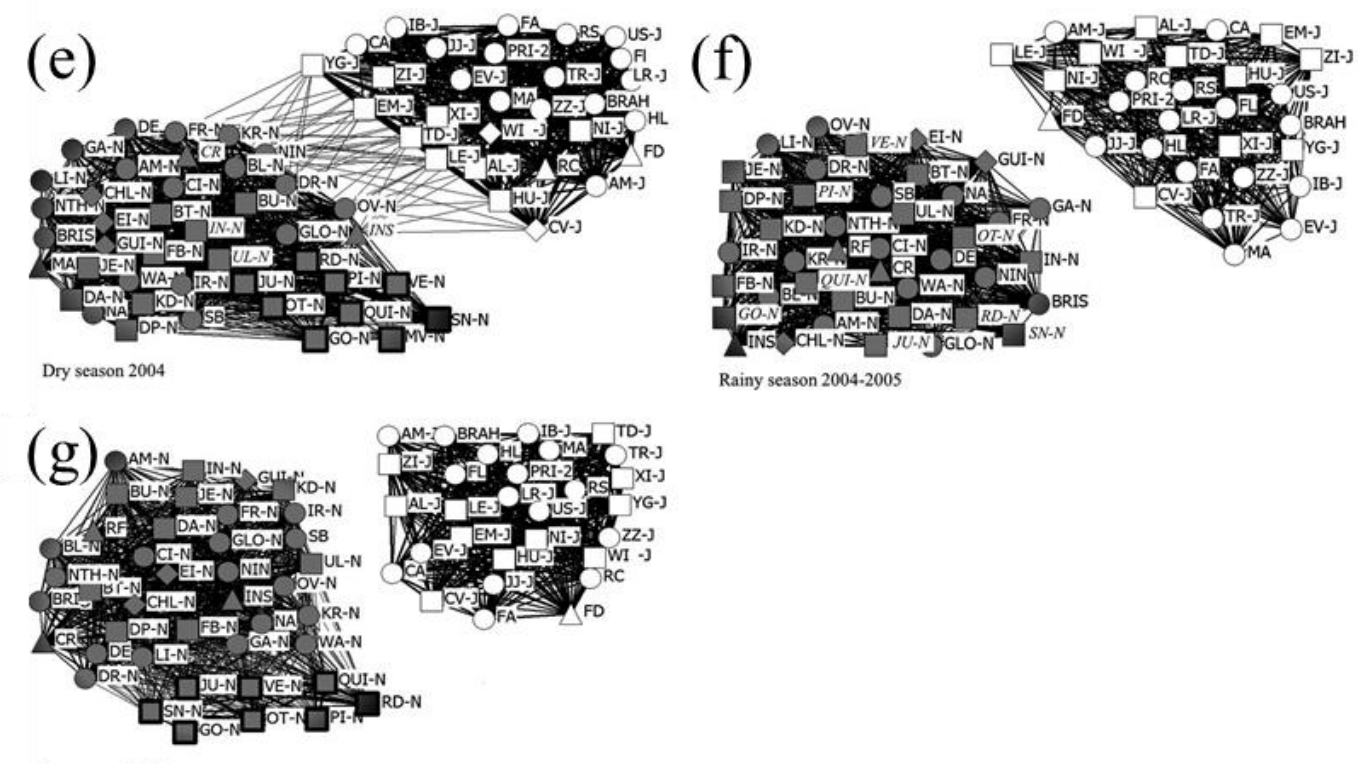

Dry season 2005 
Figure 1.8 Social networks for a northern muriqui (Brachyteles hypoxanthus) population that underwent permanent group fission between the dry season in 2002 (a) and the dry season in 2005 (g). Clusters of individuals detected by Newman's modularity method are indicated by different shading, hatching, and border width in panels (b)-(g). Circles: adult females, squares: adult males, triangles: subadult females, and diamonds: subadult males. Additional figure information can be found in Tokuda et al. (2014). Reprinted with permission from: Tokuda et al. (2014). Males follow females during fissioning of a group of northern muriquis. American Journal of Primatology, 76, 529-538. Copyright (C 2014 John Wiley and Sons. 
so for reproductive opportunities (Tokuda et al., 2014). Restructuring of the social network over time during repeated temporary fissions appears to have resulted in permanent group division; simulation studies appear to provide support for this interpretation (Sueur \& Maire, 2014).

Initiation of collective movement cannot occur without a leader. In contrast to our earlier discussion regarding leadership during collective navigation, here we will use the term leader to refer to individuals who attempt - successfully or unsuccessfully - to initiate group movement. What characteristics are possessed by successful versus unsuccessful leaders? Is leadership a stable role, consistently occupied by one or a few group members, or is it a temporary position taken up by individuals based on current knowledge or motivation? Dominance relationships or social rank appear to influence leadership in at least some species. In feral horses (Equus ferus caballus), higher-ranked individuals successfully recruited more followers when departing from the group, and horses tended to join collective movements in rank order (Krueger et al., 2014). In contrast, individual position within sociopositive social networks was not associated with leadership success. Similarly, dominance rank was strongly associated with successful initiation of collective movement in rhesus macaques (M. mulatta) (Sueur \& Petit, 2008).

Dominance rank is not always correlated with leadership success (e.g., Nagy et al., 2013). In some cases, it might be the individuals that possess the most knowledge or experience that take up leadership roles (Couzin et al., 2005; Bode, Franks, et al., 2012). In the bottlenose dolphin (Tursiops spp.) population living in Doubtful Sound, New Zealand, two particular behaviors are used to coordinate cohesive group movement on a local scale: a side-flop to initiate travel and an upside-down lobtail maneuver to signal 
cessation of movement (Lusseau, 2007). Only a subset of individuals performed these group-coordinating behaviors; side-flops were only successful when used by certain males, while upside-down lobtailing was likewise only successful when employed by particular females. By examining the social network position of signaling and nonsignaling dolphins, it was discovered that signalers had higher betweenness scores than nonsignalers: that is, signalers were more likely to associate with individuals in multiple subcommunities. Such individuals might have possessed greater knowledge regarding the likelihood that potential conspecific competitors were nearby or which resource patches had recently been visited. The possibility of eavesdropping by competitors might have resulted in greater reliance during coordination of collective movement on these local, short-distance signals compared to long-distance vocal communication.

Taken together, the above studies indicate that social network structure, as well as an individual's network position, influences individual leadership effectiveness, joining decisions, and group decision-making style in many species. While self-organization of group-level behavior can occur via local rules, it can often be misleading to assume these rules are blind to the identity of individuals with which an animal interacts. A feature worth noting in several of the above studies is the melding of model simulations with collection of empirical data on animal collective movements (e.g., Jacobs et al., 2011; Sueur et al., 2012). Through use of model simulations, predictions can be generated and then compared to empirical data in order to infer which explanation best accounts for all aspects of the data. These studies offer excellent examples of predictive approaches using SNA. Further work might incorporate ecological and social factors into the models to 
explore their potential influence on group decision-making, as well as compare explanatory models between species to assess whether similar or different behavioral mechanisms are at play.

\subsection{Animal Personalities}

Animal personality is typically defined as consistent individual differences in behavioral responses within and across contexts (Réale et al., 2007; Sih \& Bell, 2008). Animal personality research has typically focused on behavioral traits predicted to be ecologically relevant, such as aggression, boldness, sociability, activity level, and explorative tendencies (Wolf \& Weissing, 2012). Consistent between-individual differences in these and other behavioral traits have been reported for a wide range of animal taxa (Sih et al., 2010).

The existence of animal personalities is predicted to have substantial impacts on social structure and dynamics, as well as to be influenced in turn by social organization (Krause et al., 2010; Wolf \& Krause, 2014). Personality types have been shown to differ in the strength and distribution of their interactions with group members and to assort with others based on personality (e.g., Pike et al., 2008; Croft et al., 2009; Aplin et al., 2013). Some personality types can occupy prominent or influential network positions, thereby wielding disproportionate influence over network dynamics compared to other group members (Flack et al., 2006; Modlmeier, Keiser, et al., 2014).

Network metrics themselves might even be used to describe an individual's social personality if an individual consistently occupies a similar network position over time and/or across contexts (Blumstein et al., 2013; Wilson et al., 2013). In animal personality research, traits are usually measured in individuals under standardized conditions to 
ensure the trait of interest can be kept distinct from other behavioral responses, as well as to ensure the animal is presented with the same situation each time it is tested (Réale et al., 2007). Variation in these measures can then be related to behavior in naturalistic contexts. However, measurements of social behavior within isolated dyadic interactions can often be very different compared to measurements made within a realistic social environment (Krause et al., 2010; Webster \& Ward, 2011). The extent to which network measures can be used as indicators of social personality traits presents an interesting direction for future research.

Much work on animal personalities has centered on an individual's position along the bold-shy axis - that is, an individual's tendency to act in a risk-prone or risk-averse fashion (Réale et al., 2007). For example, bolder individuals are more likely to approach a novel object or investigate a potential predator (e.g., Croft et al., 2009; Kurvers et al., 2012). Variation along this axis has also been linked to fitness consequences-e.g., bolder individuals may have higher reproductive success than shy individuals, but experience decreased survival (Smith \& Blumstein, 2008). The first studies to examine the links between personality variation and network structure were done comparing network positions of bold and shy fish. Pike et al. (2008) assayed wild-caught threespined sticklebacks (G. aculeatus) in the lab for boldness - measured here as the latency to resume feeding following a mild startle response - and then formed small groups of six fish. Groups were composed of all bold individuals, all shy individuals, or a mixture of both phenotypes. In general, as the proportion of bold individuals increased in the group, mean association strength decreased and mean clustering coefficient increased. The clustering coefficient (Box 1) measures the extent to which an individual's social 
associates are themselves associated. Bold fish had weaker associations with others, but distributed those associations evenly over the rest of the group, while shy fish had a few, relatively strong, connections focused on a small number of individuals. In mixed networks, bold and shy fish showed comparable patterns as above. Differences in social network structure between groups might have been linked to the different movement patterns expressed by bold and shy individuals. Shy fish were far less likely to move if they were near a conspecific, possibly resulting in the development of strong associations between these pairs. Conversely, bold fish did not base their movement decisions on the presence of others. Similar results to Pike et al. (2008) have also been found in a wild social network of guppies ( $P$. reticulata) in that shy individuals formed stronger associations on average than did bold fish (Croft et al., 2009).

Aplin et al. (2013) explored the link between personality and social structure in a wild population of great tits ( $P$. major). Tits were outfitted with passive integrated transponder (PIT) tags that could be read by automated recording equipment set up on artificial feeders placed throughout the woods. This allowed for continuous passive monitoring of social structure in these flocks based on co-occurrence at feeding stations. A subset of the PIT-tagged population were captured and then tested in a captive environment to assess their exploration tendencies - used as a proxy for the extent to which individuals were risk-prone fast explorers or risk-averse slow explorers-before being released back into the wild. The cofeeding network derived from joint feeder arrivals demonstrated nonrandom social structure that was associated with variation in personality types (Figure 1.9(a)). Fast explorers were found to have higher degree and betweenness and weaker association strength than slow explorers: that is, fast explorers 

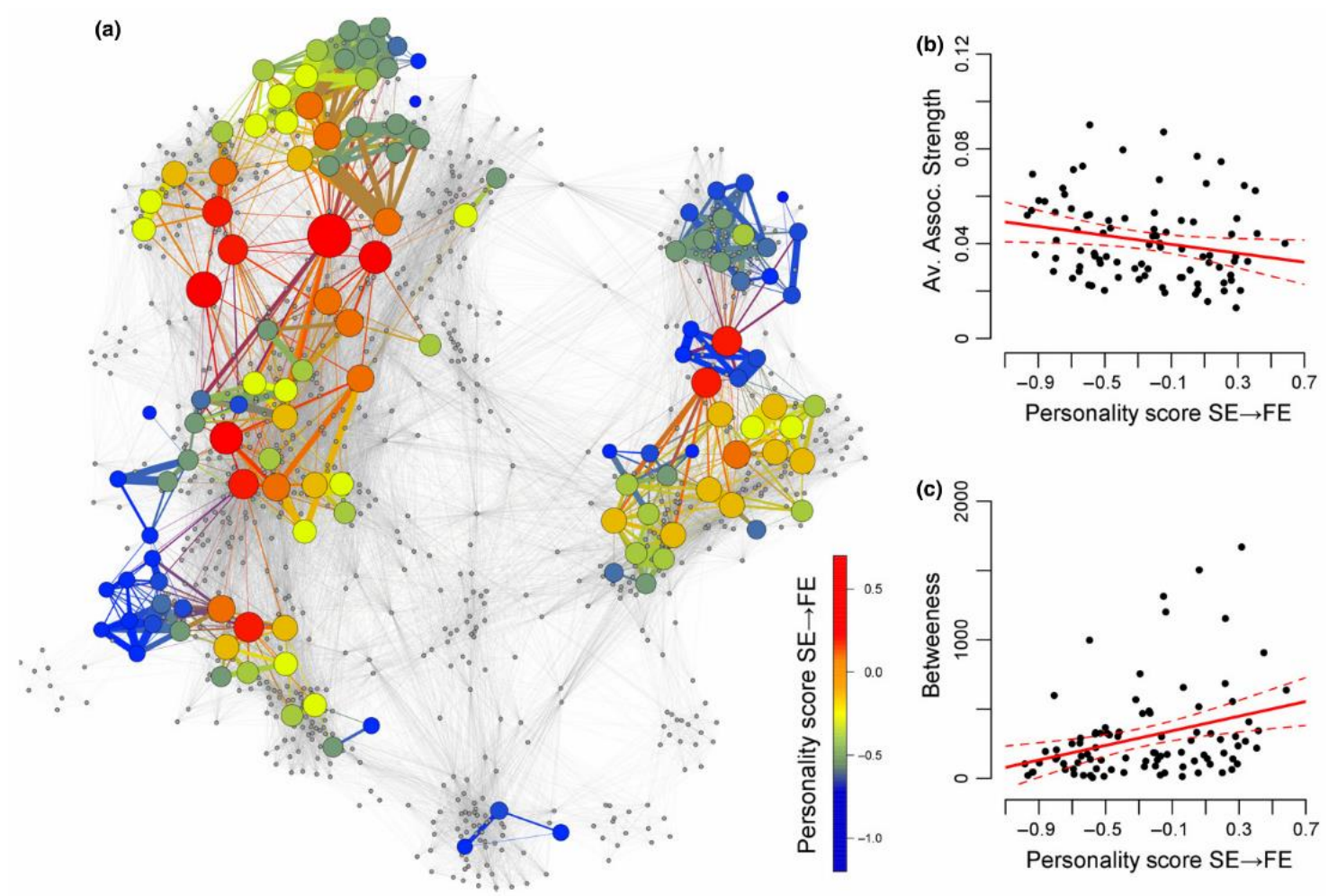

Figure 1.9 (a) A social network of great tits (Parus major) based on co-occurrence at feeding stations. Shading represents personality score ranging from risk-averse (SE) to risk-prone (FE) birds. Gray nodes indicate individuals who were not screened for personality. Node size indicates degree for birds with known personality. (b) The relationship between average association strength and personality score. (c) The relationship between individual betweenness and personality score. Dashed lines represent $95 \%$ confidence intervals. Reprinted with permission from: Aplin et al. (2013). Individual personalities predict social behaviour in wild networks of great tits (Parus major). Ecology Letters, 16, 1365-1372. Copyright (C 2013 John Wiley and Sons. 
had more numerous, but weaker social contacts than slow explorers and were more likely to move between flocks (Figure 1.9(b) and (c)). These results remained significant after controlling for differences between personality types in movement patterns and space use. Temporal stability in association patterns was also linked to personality, with slow explorers having a higher likelihood of remaining with prior associates — especially with other slow explorers — while fast explorers had more unstable associations. Bonds between two fast explorers were often especially weak and unstable. While female birds did not assort by personality, male birds preferred to associate with individuals that had similar personality types to themselves.

The work reviewed thus far has demonstrated a correlation between an individual's personality type and their network position, as well as between behavioral variation at the population level and overall network structure. Future work might explore the links between personality type and dynamic processes taking place over the network, as well as how the relative mixture of personalities within a group influences emergent phenomena. For example, prior work has demonstrated differences in how individual personality influences use of social information (e.g., Kurvers et al., 2010; Trompf \& Brown, 2014). Techniques such as NBDA could be used to explore how different personality types affect information transmission through a network. In Aplin et al.'s (2013) bird population, for example, information might flow most efficiently between slow-exploring birds and their tightly linked associates, while fast explorers might play an important role in transmitting information between different flocks. If the behavior of shy individuals results in the formation of small, tightly linked clusters, those subgroups may be buffered from exposure to pathogens, as well as find it easier to maintain 
cooperative relationships. In contrast, bolder animals could individually have faster access to new social information and thus be able to capitalize more quickly on social opportunities or obtain better access to resources. Individuals might even attempt to select or modify their social environment to best take advantage of these potential benefits or to shield themselves from social costs (e.g., Oh \& Badyaev, 2010).

A hallmark of self-organized collective movement is that if individual behavior is consistent, then collective group formations will reassemble into the same form (statistically speaking) following perturbation (Couzin et al., 2002). An intriguing parallel might exist when considering the interactions between social structure and dynamics and animal personalities. We have seen above how personality type is linked to social network structure and temporal dynamics, as well as how it might influence processes occurring over the network. We might then predict that a given mixture of personality types, whether in one group or over multiple groups, will consistently give rise to the same social structure and its associated properties, with important evolutionary implications - particularly if personality and/or network position is heritable (e.g., Dingemanse et al., 2002; Dingemanse et al., 2009; Fowler et al., 2009). Social organization can also influence the emergence of personalities, whether via frequencydependent selection, social niche specialization, or reputation building (Wolf \& Weissing, 2010). Future work might, therefore, examine whether separate populations embark on independent trajectories as their social structure influences the emergence of individual personalities and, consequently, the eventual mixture of personality types at the population level. Alternately, independent populations might instead converge on one or more stable behavioral mixtures. 


\subsection{Cooperation}

Although evolutionary biologists have been interested in cooperation and altruism ever since Darwin (Dugatkin, 2006), only recently have these subjects been addressed from a social network perspective (Nowak \& May, 1992; Nowak \& Sigmund, 1992;

Wilson et al., 1992; Nowak et al., 2010). In a widely cited study, Ohtsuki et al. (2006) found that cooperation in a structured population can persist if $b / c>k$, where $b$ is the benefit of a cooperative act received by any who are connected to the cooperator, $c$ is the cost to the cooperator for the act, and $k$ is the average degree of the network (Figure 1.10). Their "rule" indicates that cooperation is favored when individuals possess only a small number of social ties. Santos et al. (2006a) found similar results; a heterogeneous social network promoted the persistence of cooperation. This occurred even in the presence of ties that connected otherwise "socially distant" individuals, thereby allowing clusters of cooperators to be more easily invaded by defectors. Turning to real-life networks, simulations using 70 nonhuman primate social networks found that primate social structure often — though not always—-facilitated cooperation (Voelkl \& Kasper, 2009).

If cooperators are capable of assorting with other cooperators and avoiding defectors, cooperation can persist even in networks with higher levels of overall connectedness. For example, Santos et al. (2006b) found that cooperation was better able to persist when individuals were able to swiftly modify their local network in response to defection. Given that animals are often likely to have some influence over their network connections, we might expect to observe such assortative patterns in the wild (e.g., Croft et al., 2006). In general, mechanisms that allow for assortment favor the evolution of 

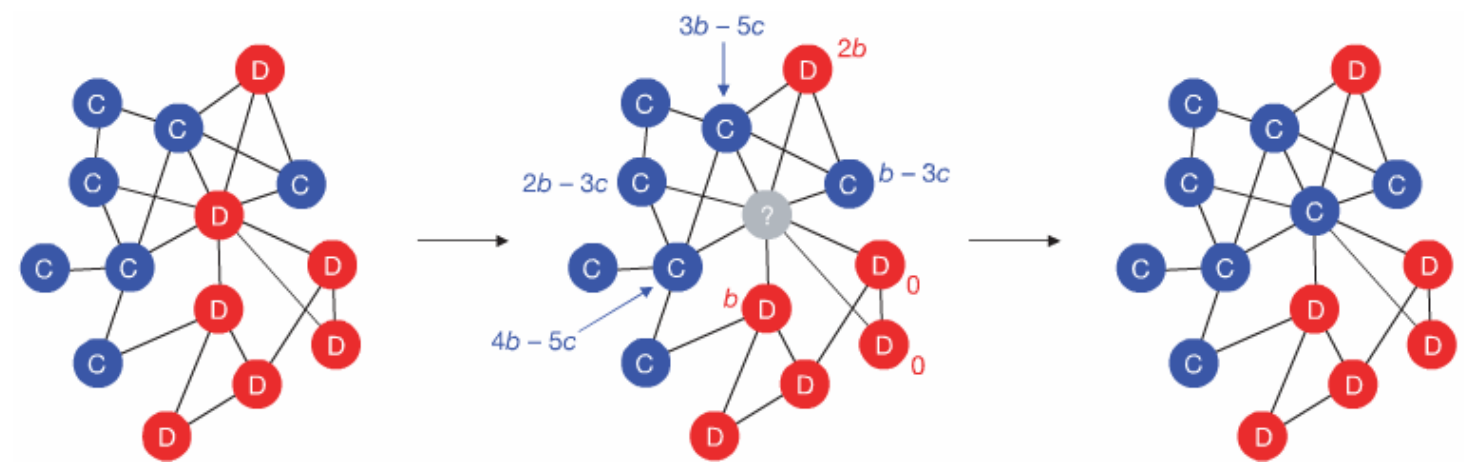

Figure 1.10 Each individual obtains a payoff $(P)$ derived from interactions with its network neighbors. Cooperators (C) pay a cost, $c$, for each neighbor to receive a benefit, $b$. Defectors (D) pay no costs, nor provide any benefits. At each time step, a random individual dies—-denoted in the figure by the node marked "?". Neighbors of the nowvacant node compete to occupy it with their offspring, with success proportional to individual fitness. Individual fitness is given by $1-w+w P$, where $w$ is the strength of selection. Reprinted by permission from Macmillan Publishers Ltd from: Ohtsuki et al. (2006). A simple rule for the evolution of cooperation on graphs and social networks. Nature, 441, 502-505. Copyright (C) 2006. 
cooperation and altruism (Wilson \& Dugatkin, 1997; McNamara \& Leimar, 2010; Nowak et al., 2010).

Cooperation can also be favored by selection when policing behavior that punishes defectors and/or maintains group stability is in place (Foster \& Ratnieks, 2001; Ratnieks \& Wenseleers, 2005). Flack et al. (2006) used SNA to study policing by male pig-tailed macaques (Macaca nemestrina). Social networks for grooming, play, contact sitting, and proximity were recorded for a captive macaque group. Subsequently, three high-ranking males who were known to engage in impartial, third-party conflict interventions were repeatedly removed from the group and social networks were again recorded. When these males were absent, aggressive behaviors became more common and affiliative behaviors less so (Flack et al., 2005). Their removal also contributed to several structural modifications in the social networks (Flack et al., 2006). Mean reachi.e., the number of nodes two or fewer steps away from the focal individual (Box 1) — and mean degree decreased for play and grooming networks, mean clustering coefficient increased for proximity networks, and macaques were more likely to assort by degree in play, grooming, and contact-sitting networks. Taken together, these structural changes suggest that in the absence of policing behavior, animals adjusted their social networks in a manner consistent with theoretical predictions regarding the maintenance of cooperation by maintaining a smaller and less diverse network of connections (Ohtsuki et al., 2006; Santos et al., 2006a). While cooperative behaviors per se were not explicitly studied by the authors, their work offers an intriguing example of how behavior can modify the social network in ways that can either facilitate or inhibit cooperative relationships. 
Coalitions involve two or more individuals cooperating with one another during potentially costly competitive or aggressive interactions (de Waal \& Harcourt, 1992). Using a long-term data set on chimpanzee (P. troglodytes) troops in Gombe National Park, Tanzania that contains both behavioral and genetic records, Gilby et al. (2013) used SNA to investigate the potential fitness benefits of coalitionary behavior for male chimpanzees. They found that reproductive success was significantly and positively correlated with individual betweenness in the coalitionary network - that is, males that had numerous coalition partners that were not themselves allied with one another sired more offspring and were more likely to rise in social rank.

Alliances, defined as long-term coalitionary relationships, are common in bottlenose dolphins (Tursiops spp.) (Connor, 1992; Connor et al., 1992; Connor et al., 1999; Wiszniewski et al., 2012). In some populations, male dolphins will form associations with one to a few other males in order to gain access to females, either by cooperatively herding them or by stealing females from other male alliances. In some cases, second- and even third-order alliances have been observed in which multiple smaller alliances join together into one superalliance (Connor et al., 1999). Wiszniewski et al. (2012) used SNA to examine alliance structure in a dolphin population near Port Stephens, Australia in which males often form strong, long-lasting bonds with one to three other males, while females have a weaker and more dispersed social structure. Male alliance composition was recorded over an 8-year period and analyzed in 2-year increments. While most males-i.e., 69-80\%-in a given 2-year period were part of an alliance, the relative stability of these alliances varied greatly. Some lasted for the entire eight years of the study, while others lasted for less than two. Wiszniewski et al.'s (2012) 
analysis found that a male's social network position was linked to alliance stability. Members of stable alliances maintained particularly tight connections within their alliance, but had very few associations outside it. Conversely, members of less stable alliances maintained a large contact network in the general population. The causes and functional consequences of this variation in alliance structure and stability, however, remain unknown.

A large body of theoretical work now exists exploring the evolution of cooperation in structured populations and providing many predictions ripe for empirical testing (see Nowak et al., 2010). Relatively less work has been done in free-living animal groups, with many questions yet to be answered. SNA provides an integrated framework that allows researchers to explore both the outcomes of cooperation on social behavior and population structure, as well as predict the likelihood of future cooperation given information about a population's social network. For example, theoretical work has associated particular structural elements with either facilitating or inhibiting the emergence and maintenance of cooperation (e.g., Ohtsuki et al., 2006; Santos et al., 2006a). The presence of these elements in animal populations allow for predictions to be made regarding the likelihood of observing cooperation. Comparative studies of social structure between species that differ in their cooperative relationships might also help to answer such questions (e.g., McDonald, 2007; Ryder et al., 2008). The outcomes of cooperative behaviors can also be studied using a social network approach. When cooperation — or a lack of it — is observed between two individuals, we might predict their social relationship will change as a result, as might their relationship with any potential eavesdroppers. Cooperation might be more likely in the presence of eavesdroppers, 
especially if those eavesdroppers are well connected. Development of dynamic network models — e.g., time-ordered networks (Blonder et al., 2012)—should shed some light on these questions by allowing us to examine how individuals shift their behavior based on social context and "rewire" their network connections over time.

Social responsiveness - the likelihood of an individual adjusting its behavior according to past interactions with particular individuals—is predicted to facilitate cooperation when past transgressions are remembered, thereby providing one mechanism by which cooperators can assort with one another and "rewire" their social ties (McNamara et al., 2009; McNamara \& Leimar, 2010). If defectors can be identified from prior direct experience, or via eavesdropping on past interactions, socially responsive individuals can adjust their behavior during future interactions with known defectors. Alternately, socially responsive individuals can facilitate cooperative behavior by threatening to terminate interactions with cheaters and seek out a more favorable social partner (McNamara \& Leimar, 2010). At the same time, personality differences between population members are predicted to result in socially responsive individuals (Wolf \& Weissing, 2010; Wolf \& Krause, 2014). Such responsiveness will only be favored if: (i) behavioral variation is present, and (ii) past behavior of a potential social partner can in part predict their future behavior-i.e., they exhibit behavioral consistency (Wolf et al., 2011). As such, the composition of personality types within a population is predicted to influence the extent to which social responsiveness is favored. This suggests the presence of at least two potential pathways by which the mixture of personality types within a population can influence cooperative behavior: (i) by influencing social network structure in ways that facilitate or inhibit cooperative behavior (e.g., Ohtsuki et al., 2006; Santos et 
al., 2006a), or (ii) by influencing the level of social responsiveness (e.g., Santos et al., 2006b; McNamara \& Leimar, 2010). However, when socially responsive individuals change their future behavior based on past interaction, this can be reflected by structural changes in their local network, as well as population-level shifts in social structure. Since social structure is also likely to influence the development of individual personalities (Wolf \& Weissing, 2010; Montiglio et al., 2013), this would suggest a potential feedback loop between social network structure, the behavioral composition of the population, social responsiveness, and the emergence or maintenance of cooperation. These potential links present an intriguing direction for future research.

\section{Future Directions for Social Network Analysis in Behavioral Ecology}

Until recently, much of SNA in nonhuman systems has dealt with how best to describe social structure in terms of interaction patterns, preferred and avoided relationships, assortment of individuals within the network, and delineating substructures within the larger global structure. Less attention has been paid to the "why" of these topics. What influences tie formation or dissolution? What ecological and social factors influence network dynamics and structure? How does social structure change over different timescales? What effect does social structure have over processes that occur on the network? What are the mechanisms by which social network position influences behavior, and what is the functional significance of both an individual's position in the network and of overall population structure? While several of the studies we have discussed in this review have begun to provide answers to some of these questions, a great deal of work remains to be done. 
A general call has recently been sounded to move SNA in behavioral ecology away from a predominately descriptive framework toward a more predictive one that seeks to explore the functional consequences of network structure and dynamics for the evolutionary ecology of social behavior (Hobson et al., 2013; Bradbury \& Vehrencamp, 2014; Pinter-Wollman et al., 2014; Wilson et al., 2014). Pinter-Wollman et al. (2014) provide an excellent review of recent advances in technology, analytical methods, and conceptual thinking in SNA. To avoid treading old ground, we refer the interested reader to their comprehensive coverage and will instead focus on two topics-comparative approaches and dynamic networks - that we feel have particular relevance for the subject areas we have addressed in this review.

\subsection{Comparative Approaches}

Comparative studies are a powerful method for assessing ecological and evolutionary hypotheses (Harvey \& Pagel, 1991). Since social structure is derived from behaviors shaped via natural selection, it is predicted to reflect selective pressures and phylogenetic history (e.g., Sundaresan et al., 2007; Kelley et al., 2011). However, network comparisons are often not straightforward, particularly when networks vary in size and connectedness (Croft et al., 2008). Many measures, such as path length and the clustering coefficient, vary with the number of nodes and edges in the network, and can be biased by sampling error. Following Croft et al. (2008), when networks of a similar size and density cannot be compared, network measures can be rescaled prior to comparison or, if the goal is to compare the network position of individuals or classes of individuals between networks, the ranks of a network measure can be used instead of its actual values. Alternately, network comparisons can make use of models that either 
control for network size, density, and other structural features (e.g., Watts \& Strogatz, 1998), or that can parameterize a network's structure so that those parameters can be compared instead between networks (e.g., Faust \& Skvoretz, 2002).

Even when measures of global network structure cannot be compared directly between networks, it can still be highly informative to compare smaller-scale network patterns and the social dynamics that inform network structure. To that end, here we briefly discuss two recently applied techniques-Markov chain network models and motif analysis - that we hope will facilitate further comparative analyses and offer fresh insights into many of the topics we have previously discussed.

Transition probabilities from Markov chain models can be directly compared between populations and species to explore how the underlying behavioral dynamics that result in social structure are influenced by ecological context and evolutionary history (Figure 1.4) (Wilson et al., 2014). While this approach is very new, it offers the tantalizing combination of allowing comparisons to be made regarding both the dynamics leading to network formation-i.e., the transition probabilities — as well as the processes that act over the network itself (see Section 3.1.3).

While these models were initially applied in behavioral ecology toward understanding shoaling decisions in freshwater fish, they can be modified and applied to other behaviors, including foraging, mating, and agonistic interactions. More complex models could incorporate multiple types of behavior at once, facilitating exploration of how behavioral processes are influenced by different forms of social behavior. For example, in principle, a model combining proximity patterns with agonistic interactions could be used to describe the effects of social eavesdropping and/or audience effects on 
dominance interactions in freely interacting animal groups. In practical terms, a different set of transition probabilities might govern agonistic interactions when individuals are in the presence of an audience and/or potential eavesdroppers as compared to when thirdparty individuals are absent. Agonistic networks could be simulated from models that take these third-party effects into account and compared with networks generated from models that ignore them to highlight their structural consequences. Comparative analyses using such models might reveal important evolutionary or ecological influences on the prevalence or importance of eavesdropping or audience effects in different environments, populations, or species. Networks generated from these Markov chain models might also be searched for structural aspects associated with the behavioral process of interest; motif analysis might prove helpful in this regard (see below).

Animals are embedded in multiple social networks, each of which might influence the others (e.g., Pearl \& Schulman, 1983; Flack et al., 2006; Barrett et al., 2012). Creating composite Markov chain models that incorporate two or more types of social behavior simultaneously—e.g., agonistic, sexual, and/or affiliative interactions—-would allow researchers to take such network dependencies into account. Markov chain models could be constructed for each behavior separately, as well as for each combination of behaviors. Networks could then be generated from these models to assess the influence of different social behaviors on population structure; comparisons with empirical data could be used to infer which behaviors were most important for driving observed patterns.

Motif analysis has recently been applied toward understanding the structural components of animal social networks (Faust, 2006, 2007, 2010; Shizuka \& McDonald, 2012; Ilany et al., 2013; McDonald \& Shizuka, 2013). Motif analysis deconstructs a 


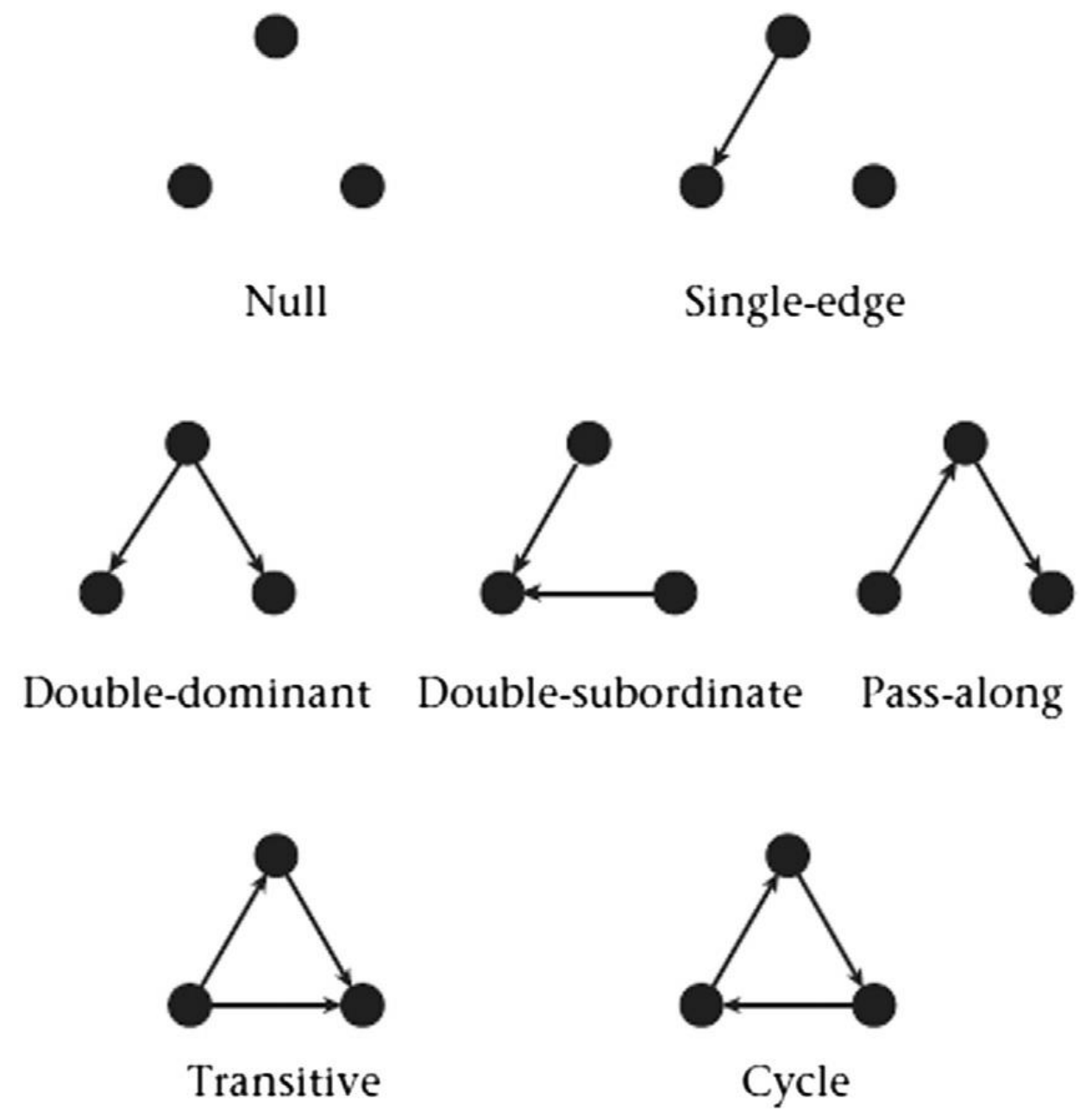

Figure 1.11 Examples of triad configurations with no symmetrical relationships-i.e., no double-headed arrows. Reprinted with permission from: Shizuka and McDonald (2012). A social network perspective on measurements of dominance hierarchies. Animal Behaviour, 83, 925-934. Copyright (C) 2012 Elsevier. 
network into subcomponents (e.g., triad configurations, see Figure 1.11), the relative frequencies of which can be compared across networks (Milo et al., 2002; Milo et al., 2004). Certain subcomponent configurations are predicted to facilitate specific network processes and properties such as information processing (Waters \& Fewell, 2012) and stable dominance hierarchies (Shizuka \& McDonald, 2012). Comparison of the relative frequencies of those configurations across populations might be used to infer the importance of certain processes in a population and their consequences for group function. Care must be taken in comparative analyses to control for differences in dyadic connection patterns, as these constrain the possible configurations for higher-level threeand four-node subcomponents (Faust, 2006, 2007, 2010); within these constraints, however, significant departures from expected frequencies can be informative. In other cases, the expected frequency of particular subcomponent configurations is independent of group size (e.g., Shizuka \& McDonald, 2012), making motif analysis an attractive option for network comparisons.

Motif analysis is best used with directed interactions (Box 1, Pinter-Wollman et al., 2014). A challenge will be to identify potential instances of directed interaction when "direction" is not obvious. In some cases, direction is clear, such as when two animals engage in an agonistic contest during which one individual emerges victorious. It is not as obvious in other cases: for example, individual A might exert influence on both individuals $\mathrm{B}$ and $\mathrm{C}$ during their agonistic interaction-e.g., A creates an audience effect. In this case, it might be difficult to identify this directed influence from A on individuals $\mathrm{B}$ and $\mathrm{C}$, particularly in freely interacting groups. Nevertheless, the emphasis motif analysis places on relationship patterns above the dyadic level—e.g., triadic — suggests it 
might be fruitfully applied toward the study of audience effects, eavesdropping, and other aspects of communication networks that are not often explicitly tackled by SNA.

Technological advances allowing for greater monitoring of signaling interactions within a wider community — e.g., microphone arrays recording songbird interactions (Foote et al., 2010) — could be especially helpful in this regard.

Motif analysis of leader-follower relationships could allow researchers to assess the extent to which the leadership hierarchy in an animal group is dominated by transitive or cyclical relationships, with potentially important implications for group function. For example, if leader-follower interaction patterns are dominated by cyclical triads, greater leadership or navigational tendencies might be required for effective group navigation. Benefits of group cohesion might select for transitive, stable leadership patterns even when no clear asymmetries exist in individual ability, experience, or information (Krause \& Ruxton, 2002; McDonald \& Shizuka, 2013). Similarly, the effectiveness of collective navigation has been linked to the frequency of particular four-node motifs (Bode, Wood, et al., 2012). Comparisons of the frequency of different leadership subcomponent configurations in various environments, or between different species, might reveal ecological or evolutionary influences on aspects of collective animal behavior.

Motif analysis is currently constrained primarily to censuses of three- and fournode subcomponents. This is because the number of possible configurations of a subcomponent increases exponentially with the total number of nodes making up that subcomponent. Analysis of subcomponents larger than about four nodes is therefore computationally prohibitive (Pinter-Wollman et al., 2014). However, as long as 
subcomponents are biologically meaningful—e.g., as when transitive triads indicate stable dominance relationships — their size may not be particularly important.

\subsection{Dynamic Networks}

A population's social network structure is rarely static, changing often as the result of demographic processes and behavioral responses to both external and internal changes. Despite widespread recognition of this fact, the dynamics of network topology have generally been neglected in the behavioral ecology literature (Blonder et al., 2012; Hobson et al., 2013; Pinter-Wollman et al., 2014). Most studies are based on a single, static network constructed from observed interactions and associations accumulated over some time span-i.e., a time-aggregated network. When temporal network dynamics have been considered, this has generally been accomplished by comparing a series of time-aggregated networks, each of which was compiled over some interval of intereste.g., seasons or years. Methods are available to study such longitudinal changes in network structure and to identify the factors influencing the probabilities of individuals changing their social relationships over time (e.g., Croft et al., 2008; Snijders et al., 2010; Pinter-Wollman et al., 2014), though these methods have been infrequently applied in behavioral ecology.

Many processes, including information flow, disease transmission, and cooperative interactions, can occur over timescales much shorter-e.g., seconds to minutes - than a longitudinal approach using time-aggregated networks can address (Waters \& Fewell, 2012). What's more, network processes and topological changes to network structure might occur over similar timescales and interact with each other in feedback loops. Knowledge of the temporal sequence of interactions is particularly 
important if one suspects that such feedback loops are at work (Blonder et al., 2012). For example, an animal that becomes infected with parasites might modify its behavior, or others might modify their behavior toward it by attempting to avoid that individual; these changes influence the subsequent likelihood of disease transmission through the population (e.g., Croft et al., 2011). This sort of feedback has been modeled using "adaptive" or "coevolutionary" networks (Gross \& Blasius, 2008), but has rarely been addressed in empirical studies on animal groups. Reciprocal feedbacks between network structure and flow dynamics might be best analyzed via time-ordered network models (Blonder et al., 2012; Pinter-Wollman et al., 2014).

Time-ordered analyses maintain data in time-stamped streams of observations, keeping a continuous record of the order, timing, and duration of interactions (Figure 1.12(a)). With this information in hand, it is possible to trace potential transmission pathways that take into account the actual order in which interactions occurred, as well as directly observe topological changes in the network (Blonder et al., 2012). Furthermore, aggregating interaction data over intervals can break the data stream down into a series of traditional time-aggregated networks (Figure 1.12(b)). Time-ordered network models are especially well suited for investigating transmission processes; researchers can use these models to: identify permitted pathways of flow, determine the relative importance of individuals in terms of their influence over these processes, and estimate the speed and efficiency of transmission within the network. While behavioral ecology has only recently begun to make use of these models (e.g., Blonder \& Dornhaus, 2011), they have been used more extensively in a number of other fields, including physics (e.g., Kostakos, 2009; Holme \& Saramäki, 2012), engineering (e.g., Kuhn \& Oshman, 2011), and the 


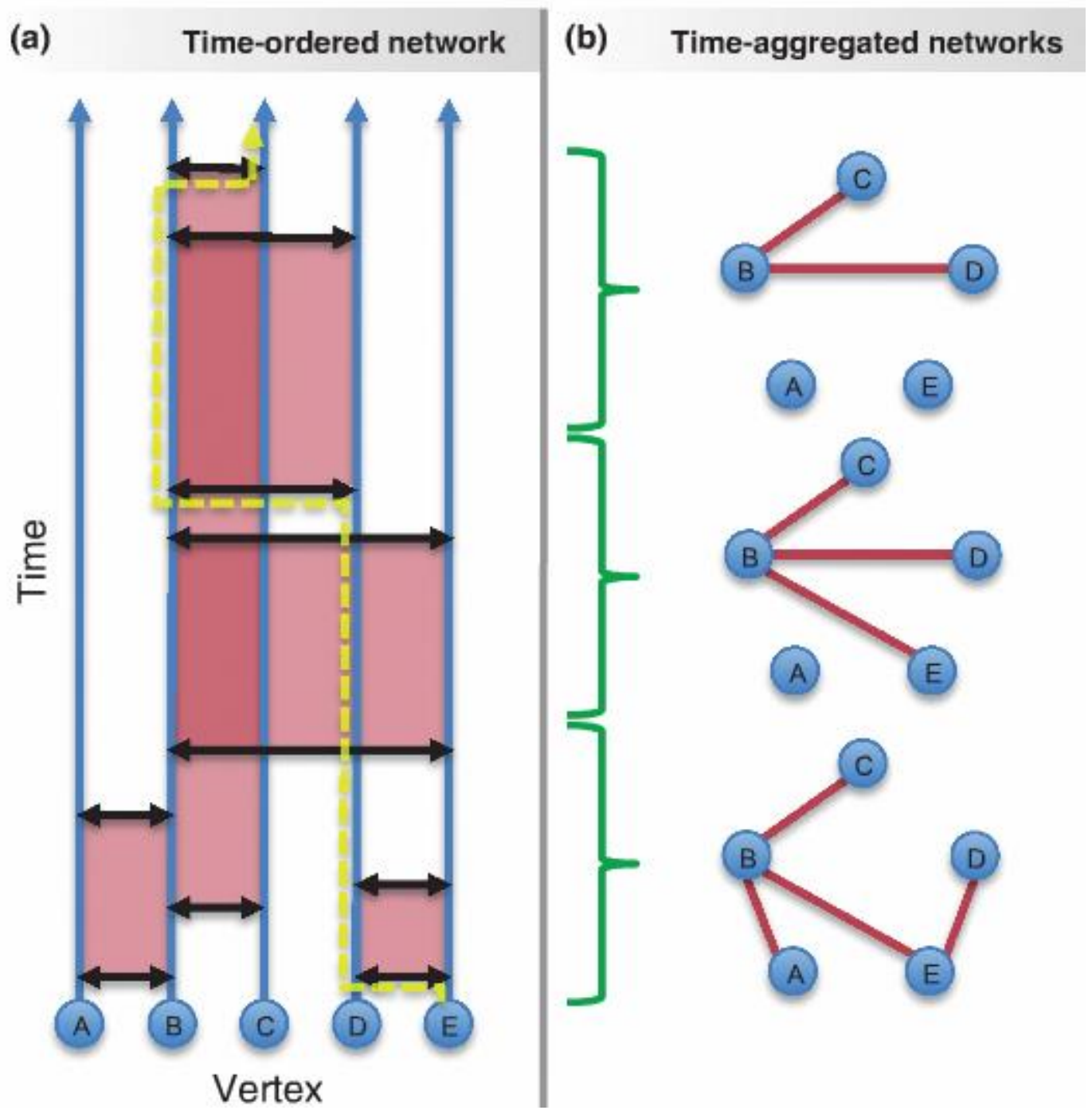

Figure 1.12 (a) A time-ordered network in which the precise sequence of interactions between individuals can be seen as time progresses. (b) Time-aggregated networks derived from the time-ordered data over specified intervals of time. Reprinted with permission from: Blonder et al. (2012). Temporal dynamics and network analysis. Methods in Ecology and Evolution, 3, 958-972. Copyright (C) 2012 John Wiley and Sons. 
computer sciences (e.g., Kempe et al., 2002; Santoro et al., 2011). Their use in behavioral ecology is likely to become more common in the future given parallel advancements in technology that allow researchers to continuously track individual animals. Social association data can now be collected automatically at high spatial and temporal resolution-e.g., via GPS devices or PIT systems (Aplin et al., 2013; Krause et al., 2013; Farine et al., 2014; Strandburg-Peshkin et al., 2014).

Time-ordered network analyses could be used to complement and offer further insight into many of the topics we have discussed in this review. For example, during collective motion and navigation, links can be formed as individuals move toward one another and dissolve as they move apart. Passive mechanisms, such as individual body condition, can influence spatial location within a moving group; if animals repeatedly interact with the same individuals during these movements, passive associations might transform into active social preferences (Bode et al., 2011a). An examination of timeordered data, as well as the time-aggregated networks that can be derived from it, could reveal whether accumulated short-term interactions during collective motion can facilitate development of more stable, long-term associations, and potentially cast light on the mechanisms by which this could occur.

Time-ordered networks might also be useful for assessing the influence of perturbations - e.g., changes in group composition, the arrival of a predator, or anthropogenic disturbances_ — on network structure and dynamics. An intriguing possibility that could be addressed with these techniques is whether there is variation in the extent to which individuals modify their social connections in response to perturbation. Some individuals might be very socially reactive, frequently and quickly 
modifying their social connections according to changing conditions or internal physiology, while others might be more socially stable and attempt to maintain the same pattern and/or intensity of connections regardless of context.

\section{Conclusion}

Starting from the musings and keen observations of naturalists and other early thinkers, the study of animal social structure and behavior has transformed over time, drawing concepts and techniques from fields as diverse as ecology, mathematics, sociology, statistical physics, evolutionary theory, and behavioral ecology. Animal SNA is now a vibrant, integrative discipline in which new insights are being generated monthly, allowing for a deeper and more holistic understanding of social structure and behavior than ever before. We have shown in this review how SNA has enriched our knowledge of behavioral ecology, as well as contributed to our understanding of many other fields. The origins of culture, the age-old problem of cooperation, and how complex, emergent group phenomena arise from individual behavior can only be fully understood when they are embedded within an explicit social context. New technologies melded with theoretical and statistical advances are expanding the horizons of SNA and taking it in novel directions at an impressive rate. We eagerly look forward to the exciting new insights sure to develop from these endeavors in the coming decades and beyond. 


\section{CHAPTER II}

\section{INTERLUDE}

Social network analysis has offered novel insights into the fine-scale structure of animal populations, the potential drivers of that structure (Sundaresan et al., 2007; Morrell et al., 2008; Aplin et al., 2013), and its consequences for social processes, such as cooperation (Croft et al., 2006), information flow (Pinter-Wollman et al., 2011; Aplin et al., 2012), disease transmission (Cross et al., 2004; VanderWaal, Atwill, et al., 2014) and sexual selection (Oh \& Badyaev, 2010). However, a frequent criticism of animal social network studies is that they are primarily descriptive without providing critical tests regarding either the mechanisms which generated the observed patterns, or the functional consequences of network structure (Pinter-Wollman et al., 2014; Wilson et al., 2014). In addition, many studies of animal networks lack replication at the network level. This is understandable, given the logistical constraints often involved, but it can limit the ability of researchers to generalize the findings of these studies to other groups or populations.

Small, shoaling fish species, such as the Trinidadian guppy (Poecilia reticulata), provide a potential means to address these concerns. Replicate social groups can be easily formed and maintained in captive environments where the ecological and social context experienced by a group can be carefully controlled. As such, small shoaling fish provide an ideal system in which to conduct manipulative experiments seeking to understand the 
determinants and consequences of social network structure.

In the following chapters, I present a series of experiments in which I manipulated the environmental conditions experienced by Trinidadian guppy shoals in order to examine their impacts on: (i) their social network structure, (ii) the fission-fusion dynamics from which that structure emerged, and (iii) the diffusion of novel foraging information through their social networks. Specifically, I manipulated the proportion of familiar group mates within a shoal (Chapter III), group personality composition (Chapter IV), and the ambient level of predation risk perceived by a group (Chapter V). In so doing, this work addresses criticisms of previous studies while contributing to our knowledge regarding the emergence and functional consequences of animal social networks. 


\section{CHAPTER III}

\section{FAMILIARITY AFFECTS NETWORK STRUCTURE AND INFORMATION FLOW IN GUPPY (POECILIA RETICULATA) SHOALS ${ }^{2}$}

\section{Introduction}

Understanding how the phenotypic composition of animal groups acts as a selective force on individual group members, although simultaneously being an emergent property of individual decision-making, has been the focus of much work within behavioral ecology (Krause \& Ruxton, 2002; Farine, Montiglio, et al., 2015). Central to this objective is determining the role played by group phenotypic composition in shaping individual fitness and group-level outcomes. Selective targeting of phenotypically different individuals by predators can promote behavioral conformity between group members (Day et al., 2001), as well as the formation of morphologically homogeneous groups that are assorted by factors such as body size, species, or sex (Krause \& Ruxton, 2002). Conversely, phenotypic diversity can be selected for by mechanisms such as social heterosis (Nonacs \& Kapheim, 2007) or negative frequency dependence (Maynard Smith, 1982); for example, in the producer-scrounger game, in which producers locate resources

\footnotetext{
${ }^{2}$ This chapter was originally published in: Hasenjager, M. J., \& Dugatkin, L. A. (2017). Familiarity affects network structure and information flow in guppy (Poecilia reticulata) shoals. Behavioral Ecology, 28(1), 233-242. It has been reprinted here by permission of Oxford University Press (C) 2017.
} 
and scroungers parasitize their efforts, scrounger success is positively associated with the frequency of producers within the group (Barnard \& Sibly, 1981).

Consistent between-individual differences in behavior represent a key component of phenotypic variation within groups. Such stable differences have been observed for numerous traits, including the propensity to use social information (Kurvers et al., 2010), leadership tendencies (Leblond \& Reebs, 2006), or averseness toward risk (Laskowski \& Pruitt, 2014; Modlmeier, Laskowski, et al., 2014). The mixture of behavioral types within a group can shape both the social decision-making of individuals (Cote et al., 2012) and the group-level outcomes (Dyer et al., 2009; Modlmeier et al., 2012; Pruitt \& Goodnight, 2014). In the social spider Anelosimus studiosus, for example, colony survival and reproductive success depends on matching the within-colony ratio of aggressive to docile individuals to the environmental conditions experienced by the colony (Pruitt \& Goodnight, 2014). In some contexts, association between unlike individuals can generate benefits for one or both parties, such as in socially foraging shoals of Trinidadian guppies (Poecilia reticulata), in which mixed shoals of bold and shy fish experienced higher foraging success than pure shoals of either behavioral type (Dyer et al., 2009).

Consistent differences in social behavior can also derive from variation in the extent to which individuals are familiar with their group mates, defined here as conditionindependent recognition of unrelated individuals (Griffiths \& Ward, 2011). Across a wide variety of taxa, familiarity has been shown to influence social preferences, including in fish (Griffiths \& Magurran, 1997; Atton et al., 2014), birds (Kohn et al., 2015), and mammals (Keller et al., 2011). Familiarity can arise through learned recognition of specific individuals (Griffiths \& Magurran, 1997; Ward et al., 2009), as well as via 
mechanisms that facilitate a more general recognition of particular classes of individual. For instance, fish can distinguish between individuals on the basis of olfactory cues derived from recent habitat use or diet (Webster, Goldsmith, et al., 2007; Ward et al., 2009).

The presence of familiar individuals within a group can have potentially large impacts on group dynamics and individual fitness. Familiarity can facilitate behavioral coordination between group members by allowing them to tailor their interactions with specific individuals based on past experience (Wolf et al., 2011). Such an effect may be responsible for the more cohesive shoaling behavior, reduced aggression, and more effective anti-predator responses exhibited by familiar shoals of fish compared with unfamiliar ones (Chivers et al., 1995; Griffiths et al., 2004; Granroth-Wilding \& Magurran, 2013). By promoting social cohesion, familiarity can also enhance the transmission of social information through a group. Guppies learned the route to a foraging site from a trained demonstrator more rapidly when the demonstrator was also familiar to them as a result of stronger tendencies to follow familiar group mates (Swaney et al., 2001). Familiarity may also generate consistent behavioral differences between group members through the process of social niche specialization, which proposes that repeated interactions between group members promote behavioral diversification due to individuals adopting different social roles in order to reduce competitive costs (Bergmüller \& Taborsky, 2010; Montiglio et al., 2013). An individual's adoption of a social role is then stabilized by positive feedback mechanisms or costs associated with role switching. Support for this hypothesis has been found in social spiders (Laskowski \& Pruitt, 2014; Modlmeier, Laskowski, et al., 2014), in which spiders exhibited more 
pronounced individual differences in boldness when they belonged to colonies in which group members were more familiar with one another; such between-individual variation has been linked to positive colony-level outcomes in both spiders and ants (Pruitt \& Riechert, 2011; Modlmeier et al., 2012).

Familiarity is generally associated with stronger social preferences between individuals. Fine-scale patterns of social structure - conceptualized as a social networkare an emergent outcome of such preferences (Wilson et al., 2014). Even within fissionfusion social systems, in which group size and membership changes frequently, social connections between familiar individuals can persist. Female brown-headed cowbirds (Molothrus ater) maintained stable social bonds with familiar females over time and across shifting group compositions (Kohn et al., 2015). Similarly, persistent social preferences were detected between females in a wild Trinidadian guppy population, and these strong network connections were correlated with greater cooperation during predator inspections (Croft et al., 2006). The structure of a social network can also shape group-level outcomes, such as the speed and pathways by which information flows through a group (Claidière et al., 2013; Aplin et al., 2015). Within three-spined stickleback (Gasterosteus aculeatus) shoals, information regarding the location of a novel foraging task was more likely to spread between familiar shoal mates than between unfamiliar ones, facilitating information transfer within — and restricting it betweenclusters of familiar individuals (Atton et al., 2014).

The influence of between-group variation in terms of familiarity on individual success and group-level outcomes has been well-studied, particularly in fish (reviewed in Griffiths \& Ward, 2011). However, despite the importance of social recognition in many 
studies of animal networks, less is known about how this variation shapes the emergent social structure of a group and how social structure might mediate these group-level outcomes (though see Morrell et al., 2008). We therefore asked whether the proportion of familiar individuals composing a group affected the fission-fusion decisions of its members, its network structure, as well as diffusion processes occurring within it. For our study, we used the Trinidadian guppy. The guppy has long served as a model system in which to investigate questions related to social information use (Dugatkin, 1992; Lachlan et al., 1998; Swaney et al., 2001; Morrell et al., 2008) and the influence of familiarity on social decision-making (Griffiths \& Magurran, 1997; Lachlan et al., 1998; Swaney et al., 2001; Ward et al., 2009; Granroth-Wilding \& Magurran, 2013). Guppies have also served as a popular study system in which to use social network analysis to probe the causes and consequences of variation in fine-scale social structure (Croft et al., 2006; Morrell et al., 2008; Croft et al., 2009; Wilson et al., 2015).

We formed groups of female guppies in which individuals were either: (i) all familiar with one another, (ii) all unfamiliar with one another, or (iii) a mixed group of familiar and unfamiliar fish. Each group was placed within an arena and the shoaling decisions of group members were recorded. Fission-fusion models of shoaling behavior were used to characterize the social dynamics occurring within each treatment and to infer the social network structure of each group (Wilson et al., 2014, 2015). Next, groups were presented with a hidden food patch and the order in which individuals discovered the patch was recorded (as in Webster et al., 2013). Network-based diffusion analysis was then used to examine whether network structure predicted information flow and to estimate the strength of social effects on patch discovery (Franz \& Nunn, 2009; Hoppitt 
\& Laland, 2013).

We tested the following predictions: (i) that individuals in groups of familiar fish would express more cohesive shoaling behavior, such that they would be less likely to remain swimming alone; (ii) that individuals in unfamiliar groups would be more likely to switch between social partners, as familiarity-based social preferences would not yet have formed; (iii) that networks based on shoaling associations would predict the diffusion of information through social groups; and (iv) that the highest diffusion rates would be observed in familiar groups.

\section{Methods}

\subsection{Study Subjects and Rearing Conditions}

Study subjects were lab-reared descendants of wild-caught fish collected in 2003 from the Quaré River in Trinidad. The study was conducted in two replicate blocks from February to June 2014. Juvenile guppies (ca., $\leq 7 \mathrm{~mm}$ in length) were collected from four 208-L stock tanks, each of which contained several hundred fish. To reduce the likelihood of social preferences expressed during the experiment being systematically influenced by kinship or by familiarity developed within the stock tanks early in life, juveniles were randomly assigned to one of the fifteen 37.9-L rearing tanks and were reared together in groups of 24 fish. Rearing tanks were visually and chemically isolated from one another, filtered, and maintained at $26-27^{\circ} \mathrm{C}$ on a $12-\mathrm{h}: 12-\mathrm{h}$ light-dark cycle. Fish were fed TetraMin® Tropical Flakes daily.

Once fish had matured, six size-matched, non-gravid females were selected from each rearing tank for the experiment; at this point, population sizes varied between rearing tanks due to mortality (mean \pm standard deviation (SD): $21 \pm 2.2$ individuals). All 
individuals besides the selected females were then returned to the stock tanks. We used female guppies because they are more likely to express persistent social preferences (Griffiths \& Magurran, 1998; Croft et al., 2006) as well as tend to be more food motivated than males (Reader \& Laland, 2000). At the time of testing, rearing groups had been together for 51 to 55 days. Learned familiarity can develop in guppies under captive conditions in as little as 12 days (Griffiths \& Magurran, 1997), so we considered fish reared in the same tank to be familiar with one another and fish reared in separate tanks to be unfamiliar. Olfactory cues originating from the unique chemical mélange within each rearing tank may also have contributed to social recognition (Ward et al., 2009). To permit individual identification, each female was anesthetized with MS-222 and injected with a combination of two colored elastomer tags. This method has been extensively used in guppies (e.g., Croft et al., 2006; Morrell et al., 2008; Croft et al., 2009; Wilson et al., 2015) and does not appear to influence shoaling decisions (Croft et al., 2004). Females recovered quickly after marking and no mortalities occurred as a result of this procedure.

\subsection{Testing Procedure}

Prior to testing, rearing groups were habituated to the test arena; at the start of habituation, rearing groups had been together for 40 days. The test arena was a black rectangular tank measuring $62 \times 44 \mathrm{~cm}$ with black gravel substrate and water $5 \mathrm{~cm}$ deep (Figure 3.1). Black cloth was hung around the arena setup to minimize disturbance from any visual cues in the room. Each rearing group was released within the arena for 30 minutes on five separate occasions. Each time, flake food was sprinkled over the water's surface in sufficient amounts to minimize competition, as judged by the presence of uneaten flake food at the end of each session. The arena and gravel were rinsed and 


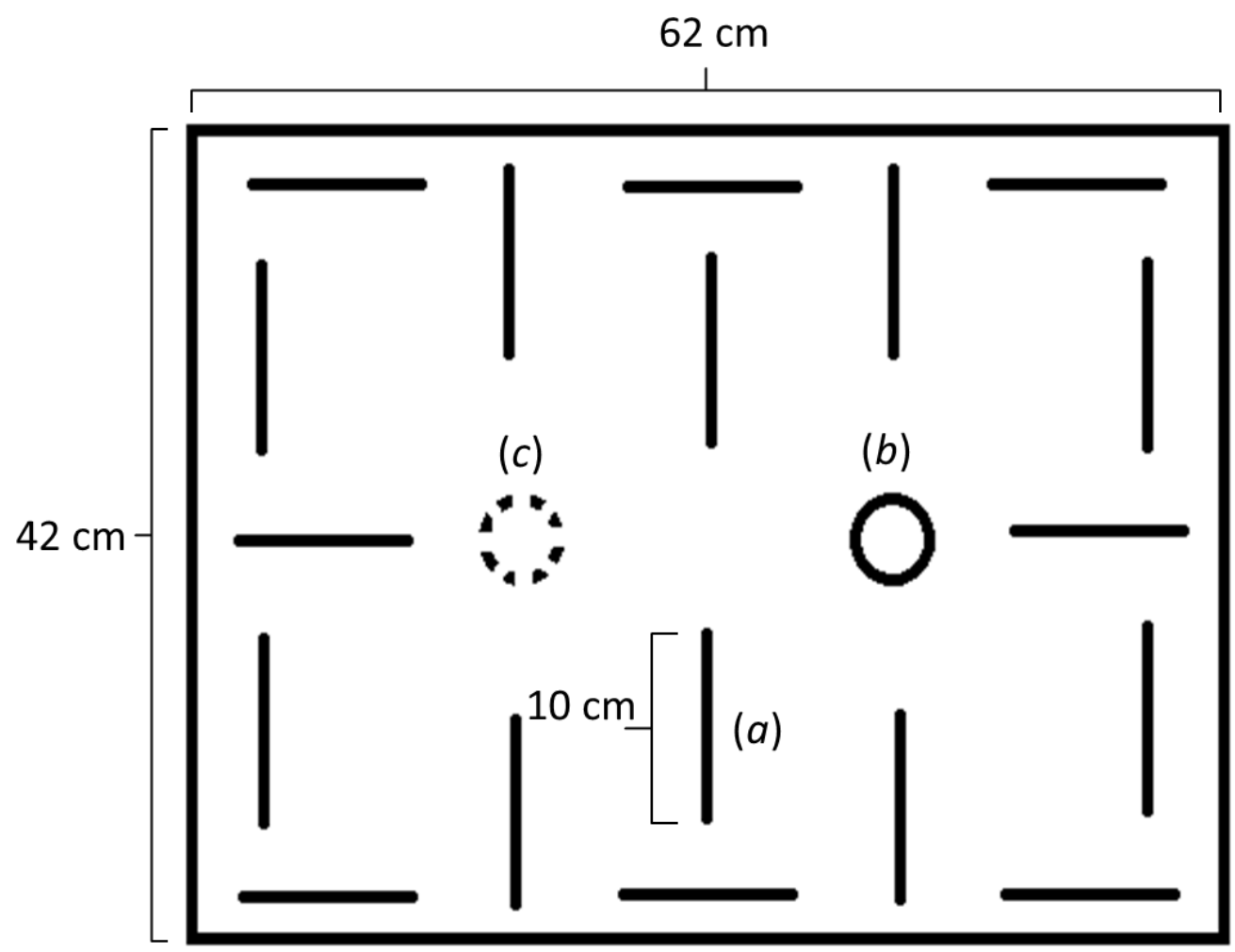

Figure 3.1 The test arena measured $62 \times 44 \mathrm{~cm}$ and contained black gravel and water 5 $\mathrm{cm}$ deep. Eighteen $10 \mathrm{~cm}^{2}$ partitions (a) were used to create a structurally complex space. Two patches were located at the arena center: (b) a sealed, transparent container with flake food recessed into the gravel and (c) an area of gravel indistinguishable from the surrounding substrate and identical in size to the food patch. These patches were concealed from groups during the initial 120 minutes in the arena. 
scrubbed between subsequent uses. Habituation sessions for a rearing group occurred every other day for a 10-day span. Testing began on the day after the final habituation sessions.

Three treatment groups were tested each day. Our study had three treatments: (i) familiar ( $n=9$ groups), in which all six fish originated from the same rearing group, (ii) unfamiliar ( $n=10$ groups), in which all six fish originated from separate rearing groups, or (iii) mixed ( $n=10$ groups), in which three fish were familiar with one another, whereas the remaining three originated from three separate rearing tanks. Unfamiliar and mixed groups were formed immediately prior to being transferred to the arena. One familiar group was lost due to mortality unrelated to the study. The mean $( \pm$ SD) standard length of fish in this study was $16.9 \pm 1.4 \mathrm{~mm}$; within each group, all individuals were within $3 \mathrm{~mm}$ standard length of one another. This was done to minimize the impact of size-based preferences (Krause \& Ruxton, 2002; Wilson et al., 2014) in order to better isolate any influence of familiarity on shoaling decisions.

The testing procedure was adapted from Webster et al. (2013). The arena was as described above, with the addition of eighteen $10 \mathrm{~cm}^{2}$ partitions set up perpendicular to one another to break up sight lines between fish in the arena (Figure 3.1). The foraging and control patches were located at the arena center. The foraging patch was a transparent cylindrical container $(3.5 \mathrm{~cm}$ diameter $)$ containing flake food that was recessed into the gravel and sealed to prevent release of olfactory cues; fish had to pass over the foraging patch in order to locate it. An area of substrate identical in size to the foraging patch, but containing no food and possessing no topological features distinguishing it from the surrounding gravel, was designated as the control patch. Two black cylinders $(10.5 \mathrm{~cm}$ 
diameter) concealed both patches from groups for the first 120 minutes in the arena. Overhead illumination was provided by four $23 \mathrm{~W}$ compact fluorescent lights. Groups were filmed by a Canon Vixia HG21 camcorder suspended $1.2 \mathrm{~m}$ above the arena.

Groups were given 30 minutes of acclimation before being recorded for 90 minutes to collect shoaling association data. Focal individuals were randomly selected and observed continuously for 4 minutes. Every 10 seconds, we recorded whether the focal fish was asocial — that is, no group mates were within four body lengths—or social. If the latter, we recorded the identity of its nearest neighbor as measured from the center of their heads. We also recorded activity based on whether the focal had moved $\geq 10 \mathrm{~cm}$ since its prior observation. After 4 minutes, a new focal fish was randomly selected until all group members had been observed. This process was repeated twice more, allowing 6 minutes to elapse between consecutive sessions, to provide 72 observations per individual. After this 90 minute period, we gently removed the cylinders to reveal the foraging and control patches. Groups were filmed for an additional 30 minutes, during which we recorded the time of first arrival for each individual at both the real and control patch for use in the network-based diffusion analysis. The arena, gravel, and all items within the arena were scrubbed and rinsed on trial completion.

All experimental procedures, as well as animal care and maintenance protocols, were approved prior to the study's commencement by the University of Louisville's Institutional Animal Care and Use Committee (IACUC \#13020).

\subsection{Fission-Fusion Dynamics and Social Network Structure}

To characterize the social dynamics acting within our treatments, we used the fission-fusion model of shoaling behavior originally developed by Wilson et al. (2014). 
The shoaling behavior of a focal individual is described by a sequence of behavioral states, where swimming alone is denoted by $a$ and shoaling is denoted by $s_{g}$, where $g$ is one of $k$ potential nearest neighbors (Figure 3.2). If $a$ and $s_{1} \ldots s_{k}$ are regarded as states of a first-order Markov chain, then the probability of transitioning between states is determined solely by an individual's current state. Transition probabilities were estimated as simple proportions from the observed sequences of shoaling behavior. Three individuals, two from mixed groups and one from an unfamiliar group, were not visible throughout their trial. Focal follows were conducted on 54 fish in familiar groups, 58 fish in mixed groups, and 59 fish in unfamiliar groups.

The model can be characterized by the probabilities of an individual switching from being alone to shoaling $[p(a, s)]$, of ending social contact $[p(s, a)]$, and of switching between nearest neighbors though remaining social [p(switch)] (Figure 3.2). The probability of a focal individual maintaining its current state is determined by the respective transition probability-for example, $p(a, a)=1-p(a, s)$. As some behavioral states—-for example, swimming alone—-were not observed with sufficient frequency to permit accurate estimation of individual-specific transition probabilities, data were pooled across all focal follows within each group. Weighted mean transition probabilities were then calculated for each treatment, where weighting was based on the relative frequency of opportunities for state transitions within a group. Nonparametric bootstrapping was used to generate $95 \%$ confidence intervals (CIs) using the boot package (Davison \& Hinkley, 1997; Canty \& Ripley, 2016) in R version 3.2 (R Core Team, 2016).

To test whether fish expressed social preferences for particular individuals, we 


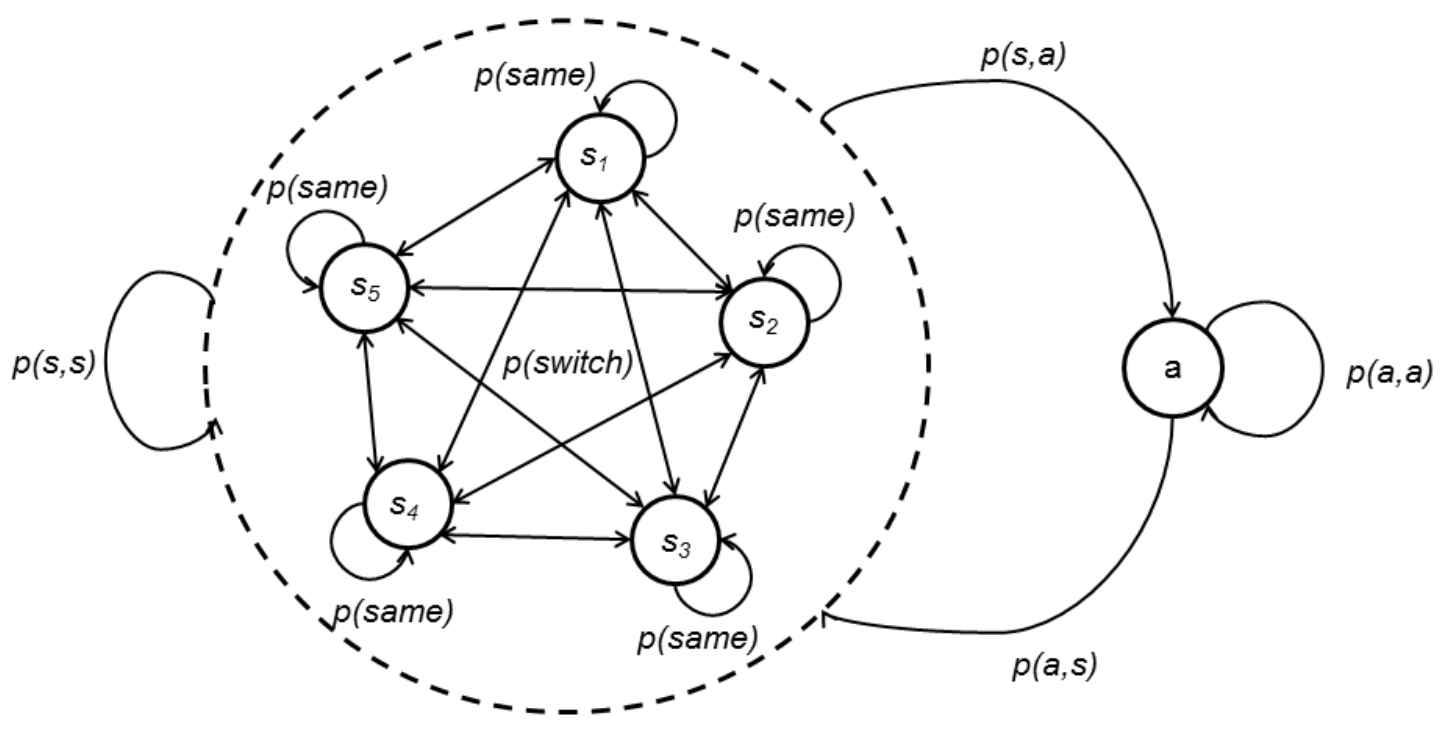

Figure 3.2 Markov chain model of shoaling behavior. A focal individual can either be swimming alone - $a$ - or shoaling with some nearest neighbor- $s_{g}$ - where $g$ is one of $k$ potential nearest neighbors. A focal individual that is alone remains so during the next observation with probability $p(a, a)$ or will begin shoaling with another individual with probability $p(a, s)$. An individual shoaling with a nearest neighbor will either continue shoaling with that individual at the next observation with probability $p($ same), begin shoaling with a different nearest neighbor with probability $p$ (switch) or cease shoaling entirely with probability $p(s, a)$. This model does not include individual-specific nearest neighbor preferences; when an individual switches nearest neighbors, it selects its new partner with probability equal to $p($ switch $) /(k-1)$. The probability of maintaining shoaling behavior rather than beginning to swim alone is given by $p(s, s)$. 
first quantified the association strength between each pair of group mates in terms of: (i) the average duration of a contact phase between them and (ii) the total number of distinct contact phases with each other. A contact phase between two individuals was one or more consecutive observations in which they were recorded as being nearest neighbors to one another. We then employed randomization tests where, for each focal individual, we kept constant their observed number of contact phases and their lengths but randomized the identities of their nearest neighbors prior to recalculating association strengths using both methods described above. This randomization procedure was carried out $10^{4}$ times. The test statistic was the sum of squares of the association strength across all pairs within a group. If the observed test statistic was in the top $5 \%$ of the distribution generated by the randomization procedure, this was used as evidence that focal fish were expressing individual-specific nearest neighbor preferences. We then calculated combined $P$ values using Fisher's omnibus procedure to examine treatment-level patterns (Haccou \& Meelis, 1994).

Using networks based on the total number of contact phases between pairs (see Results), we tested whether a pair's association strength was correlated with their similarity in terms of body length, activity level, or shoaling tendency using Mantel tests. Activity level and shoaling tendency were calculated as the proportion of observations in which a focal individual was recorded as being active or shoaling respectively. Similarity was calculated by subtracting the difference between two individuals in terms of the variable of interest from the maximum value possible - for example, for body length, this was the maximum length measured within a group. Similarity matrices for each group were then constructed from these values. Two-tailed Mantel tests were conducted in 
SOCPROG version 2.5 with $10^{4}$ permutations per test (Whitehead, 2009). The $P$ values within a treatment were subsequently combined using Fisher's omnibus procedure (Haccou \& Meelis, 1994).

\subsection{Network-Based Diffusion Analysis}

We used the order-of-acquisition diffusion analysis (OADA) variant of networkbased diffusion analysis (NBDA) to examine the rate and pattern of information diffusion in our groups (Hoppitt \& Laland, 2013). OADA examines whether the order in which individuals acquire information is predicted by their social network. The time of first arrival at a patch was used to indicate when an individual became knowledgeable about its location. A general form of the NBDA model, extended to consider diffusions in separate groups, is given by:

$$
\lambda_{i k}(t)=\lambda_{0}(t)\left(1-z_{i k}(t)\right)\left(s \sum_{j=1}^{N_{k}} a_{i j k} z_{j k}(t)+1\right)
$$

in which $\lambda_{i k}(t)$ is the rate at which individual $i$ in group $k$ discovers the patch at time $t, \lambda_{0}$ is the baseline rate of acquisition, $z_{i k}(t)$ is the informational status of individual $i$ in group $k$ at time $t$ where $z=1$ is informed and $z=0$ is naive, $s$ is the fitted social effect parameter, and $a_{i j k}$ is the association strength between individuals $i$ and $j$ in group $k$. We used an OADA variant modified to allow between-group comparisons; for additional details, see Hoppitt and Laland (2013).

We tested the explanatory power of two network types: (i) association networks, where the association strength between each pair was based on the total number of contact phases between them, and (ii) homogeneous networks, where the association strength between each pair was set to 1 . The former tests whether the strength of social 
effects on patch discovery is proportional to the association strength between individuals, whereas the latter tests whether social effects operate homogeneously throughout each group.

Comparison of social effect strength between the real and control patch allowed us to assess the level of support for social transmission relative to that for untransmitted social effects. Social transmission refers to informed individuals increasing the probability of naive individuals becoming informed due to the former's behavior (Hoppitt \& Laland, 2013). In contrast, when information or a trait can only be acquired at a specific location — as in our study_-individuals who frequently associate together might also be likely to acquire this information at similar times to one another through otherwise asocial means - that is, an untransmitted social effect (Atton et al., 2012; Webster et al., 2013). For example, individuals traveling together could simultaneously encounter a novel food patch. Although social transmission and untransmitted social effects could act together to affect the discovery of a food patch, we assumed that only untransmitted social effects would affect the diffusion of 'knowledge' regarding the location of an arbitrary area of gravel — that is, the control patch. Under this assumption, the social effect parameter, $s$, for the real diffusion is equal to $s_{T}+s_{U}$, where $s_{U}$ is the social effect estimated for the control diffusion and $s_{T}$ estimates the potential strength of social transmission.

We compared the explanatory power of four parameterizations that establish how the social effect parameter, $s$, might vary within and between treatments: (i) social effect strength could vary between treatments - for example, allowing for faster diffusion rates in some treatments - as well as vary between real and control patches; (ii) social effect 
strength could be equal across all treatments, but vary between patch types; (iii) social effect strength could vary between treatments, but not between patch types; or (iv) social effect strength could be equal across all treatments and patch types. The first two alternatives allow for social transmission, whereas the latter two suggest that primarily untransmitted social effects may be at work.

We also included several individual-level variables to examine their influence on baseline rates of patch discovery. For each individual, we included its body length and the number of days since its last habituation session. Both variables were first standardized. We also included variables that allowed the discovery rate to vary between patch types, between replicate blocks, and for patch location - that is, the left or right side of the arena. Both additive and multiplicative OADA variants were considered; these specify how individual-level variables interact with social effects. Models were fitted for every combination of network type, OADA variant, social effect parameterization, and individual-level variables. An additional model set was fitted that allowed for only asocial learning but permitted patch discovery rates to differ between treatments. Diffusion data could not be collected for one unfamiliar group due to a malfunction with the foraging patch that resulted in the food not being visible; analyses were conducted on 9 familiar, 10 mixed, and 9 unfamiliar groups.

Akaike weights_-based on Akaike's information criterion corrected for sample size - were calculated for each fitted model, indicating the support provided by the data for a model relative to the rest of the model set (Burnham \& Anderson, 2002). The total support_-given as a \% - for each network type, OADA variant, social effect parameterization, and individual-level variable was then obtained by summing the 
Akaike weights for each model in which it was included over the complete model set. Model-averaging approaches were used to calculate parameter estimates and confidence intervals. For the individual-level variables, we calculated 95\% confidence intervals adjusted to account for model selection uncertainty (Burnham \& Anderson, 2002). Confidence intervals for social effect parameters are often asymmetric (e.g., Webster et al., 2013); thus, these were obtained using profile likelihood techniques adjusted for model selection uncertainty (Burnham \& Anderson, 2002; Morgan, 2009). Confidence intervals were also obtained for the estimated differences in social effect strength between treatments. OADA was conducted in R version 3.2. (R Core Team, 2016) using code (version 1.2.11) freely available at http://lalandlab.st-andrews.ac.uk/freeware.

\section{Results}

\subsection{Fission-Fusion Dynamics and Social Network Structure}

Observed fission-fusion dynamics were well reflected by our models as assessed by comparing the observed distributions of the lengths of social contact, of contact with a particular nearest neighbor, and of being alone with simulated distributions generated using the estimated transition probabilities for each treatment (Figure 3.3). The proportion of familiar individuals within a group did not influence its overall fissionfusion dynamics, as transition probabilities did not significantly differ between treatments (Figure 3.4).

Social preferences were not expressed in the average duration of contact between two individuals (Omnibus tests: familiar: $\mathrm{X}^{2}=11.99, \mathrm{df}=18, P=0.848$; mixed: $\mathrm{X}^{2}=$ 9.02, $\mathrm{df}=20, P=0.983$; unfamiliar: $\left.\mathrm{X}^{2}=21.85, \mathrm{df}=20, P=0.349\right)$. When association strength was quantified as the total number of distinct contact phases between a pair, 

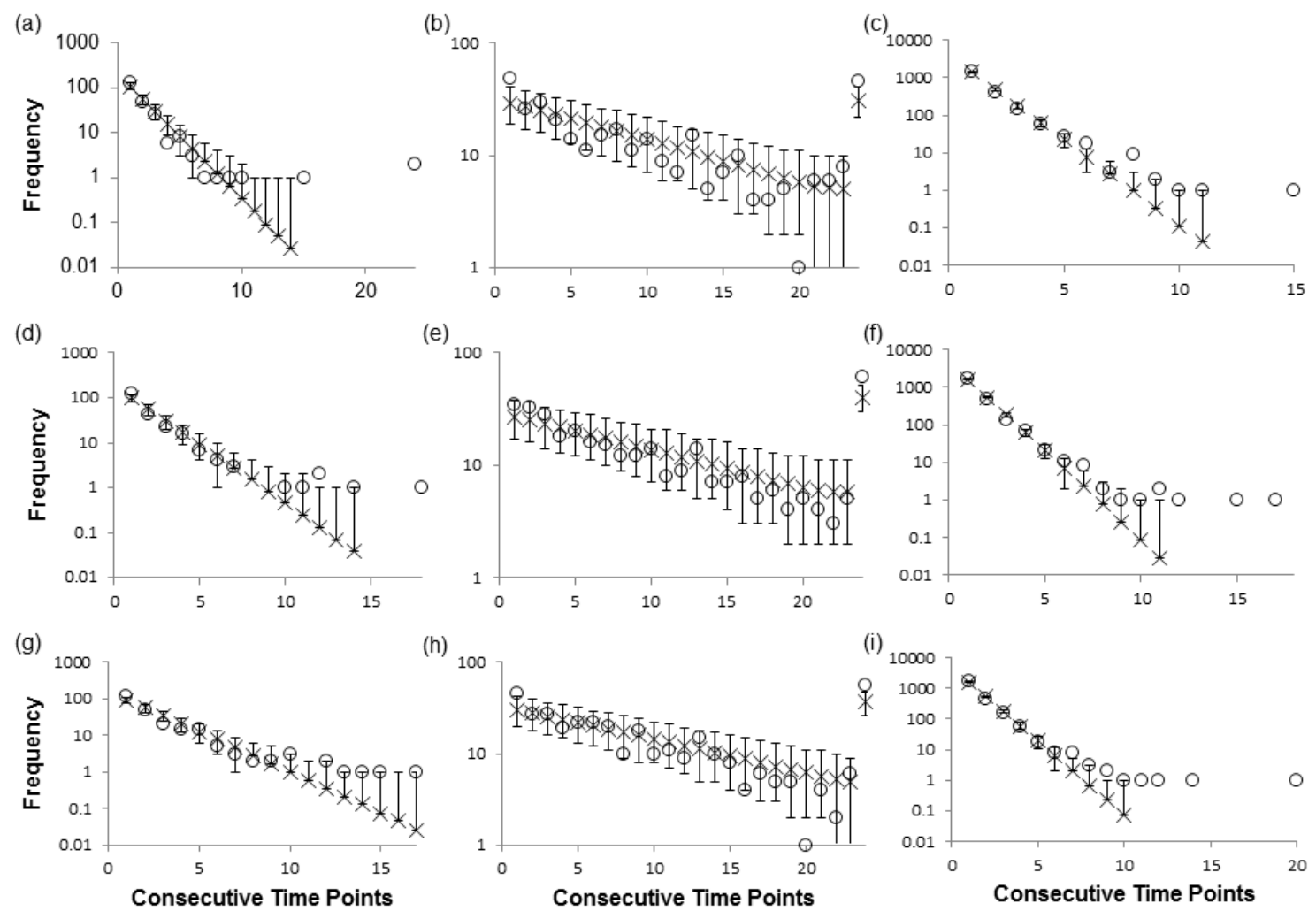

Figure 3.3 Frequency distributions of (a) the length of time being alone, (b) the length of continuous social contact, and (c) the length of contact with a particular nearest neighbor for groups composed entirely of familiar individuals ( $n=9$ groups). Observed data are depicted as circles. Using weighted mean transition probabilities, we generated $10^{4}$ simulations of our Markov chain model. The mean values ( $x$ 's) and the $2.5 \%$ and $97.5 \%$ percentiles obtained from these simulations are depicted. Values of 0 cannot be displayed on a logarithmic plot and are omitted. For each simulated run, the observed starting states for focal individuals and the four-minute observation time per focal follow were maintained. The corresponding data for mixed groups ( $n=10$ groups), (d)-(f), and for unfamiliar groups ( $n=10$ groups), (g)-(i), are also depicted. 


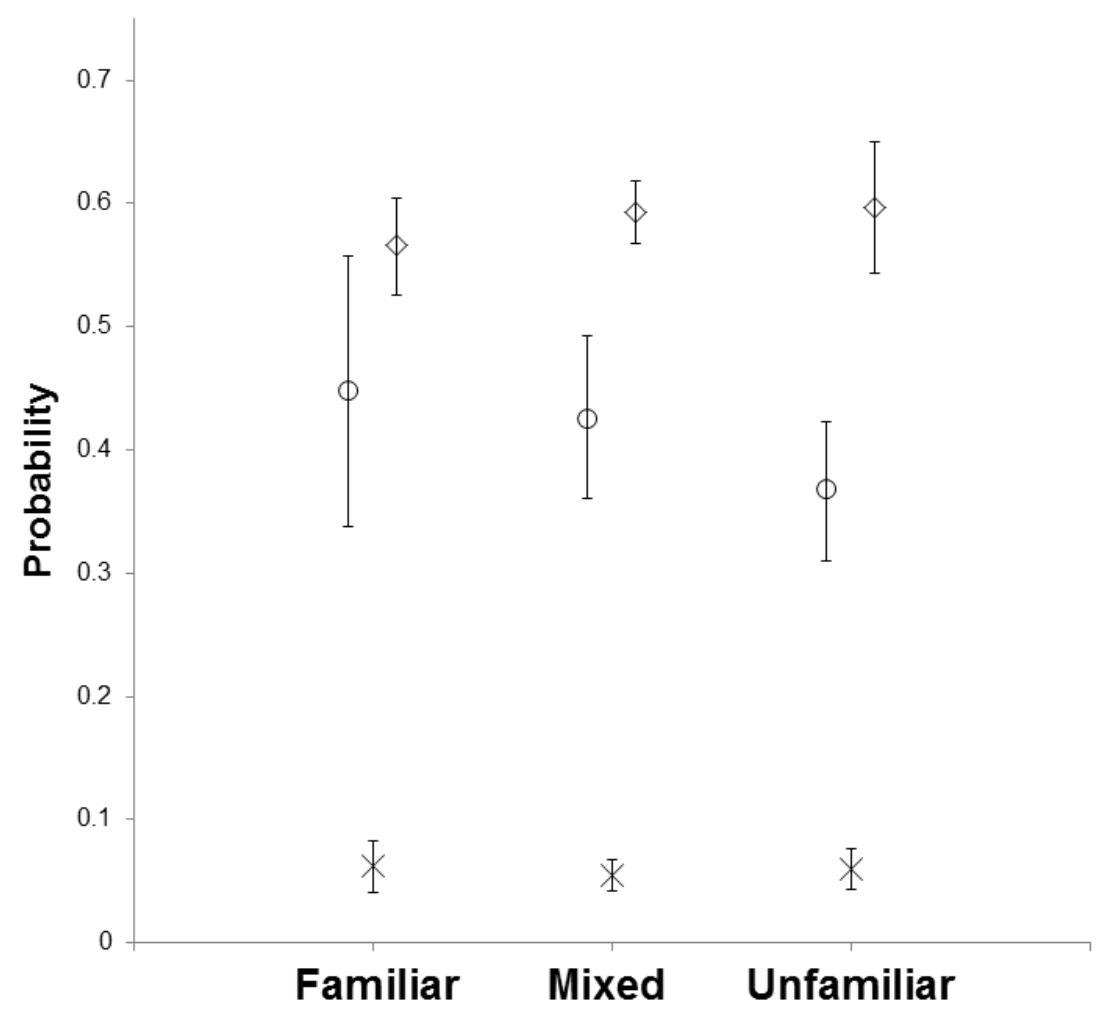

Figure 3.4 Transition probabilities for Markov chain models of shoaling behavior. Weighted means and 95\% confidence intervals are shown (number of groups within each treatment: familiar $=9 ;$ mixed $=10 ;$ unfamiliar $=10)$. Circles represent the probability, $p(a, s)$, of starting to shoal after being alone, $x$ 's represent the probability, $p(s, a)$, of ending social contact to swim alone, and diamonds represent the probability, $p$ (switch), of switching between nearest neighbors. 
guppies exhibited nonrandom partner selection in familiar (Omnibus test: $X^{2}=31.54$, $\mathrm{df}=$ $18, P=0.025)$ and unfamiliar groups (Omnibus test: $\mathrm{X}^{2}=36.02$, $\mathrm{df}=20, P=0.015$ ).

Partner selection in mixed groups did not differ from random expectations (Omnibus test: $\left.\mathrm{X}^{2}=26.11, \mathrm{df}=20, P=0.162\right)$.

Mantel tests detected no correlation in familiar groups between association strength and phenotypic similarity in terms of body length (2-tailed Omnibus test: $\mathrm{X}^{2}=$ 11.48, $\mathrm{df}=18, P=0.873$ ), activity level (2-tailed Omnibus test: $\mathrm{X}^{2}=20.81, \mathrm{df}=18, P=$ 0.289), or shoaling tendency (2-tailed Omnibus test: $X^{2}=19.08$, df $\left.=18, P=0.387\right)$. In mixed groups, association strength was correlated with similarity in terms of shoaling tendency (2-tailed Omnibus test: $\mathrm{X}^{2}=42.39$, $\left.\mathrm{df}=20, P=0.002\right)$ but not body length $(2$ tailed Omnibus test: $\mathrm{X}^{2}=22.19$, $\mathrm{df}=20, P=0.330$ ) or activity level (2-tailed Omnibus test: $\left.X^{2}=18.61, \mathrm{df}=20, P=0.547\right)$. In unfamiliar groups, there was a nonsignificant tendency toward a positive correlation between association strength and similarity in terms of activity level (2-tailed Omnibus test: $X^{2}=31.50, \mathrm{df}=20, P=0.049$ ) but not in terms of shoaling tendency (2-tailed Omnibus test: $\mathrm{X}^{2}=30.15$, $\mathrm{df}=20, P=0.067$ ) or body length (2-tailed Omnibus test: $\left.\mathrm{X}^{2}=11.21, \mathrm{df}=20, P=0.941\right)$.

\subsection{Network-Based Diffusion Analysis}

Models that included social effects on patch discovery were strongly favored over models that only allowed for asocial learning, with the latter receiving virtually no support (Table 3.1). Association networks based on the total number of contact phases between group members predicted the order in which individuals discovered patch locations (94.7\% support). For social effect parameterizations, the greatest support was found for social effects differing between treatments but not between patch types $(58.6 \%$ 
Table 3.1 Total support (\%) based on Akaike weights for network types and OADA variants

\begin{tabular}{|c|c|c|}
\hline Network & \multicolumn{2}{|c|}{ Total Support } \\
\hline \multirow[t]{3}{*}{ Asocial learning only } & \multicolumn{2}{|c|}{$0 \%$} \\
\hline & \multicolumn{2}{|c|}{ OADA variant } \\
\hline & Additive & Multiplicative \\
\hline Association network & $76.04 \%$ & $18.70 \%$ \\
\hline Homogeneous network & $4.52 \%$ & $0.74 \%$ \\
\hline
\end{tabular}

Table 3.2 Total support (\%) based on Akaike weights for social effect parameterizations

\begin{tabular}{lc}
\hline Parameterization & Total Support \\
\hline Treatments equal; real patch $=$ control patch & $23.51 \%$ \\
Treatments differ; real patch $=$ control patch & $58.57 \%$ \\
Treatments equal; real patch $\neq$ control patch & $12.61 \%$ \\
Treatments differ; real patch $\neq$ control patch & $5.31 \%$ \\
\hline
\end{tabular}


Table 3.3 Model-averaged parameter estimates, confidence intervals, and \% support ${ }^{a}$

\begin{tabular}{llccc}
\hline & df & & $\begin{array}{c}\text { Parameter } \\
\text { estimate }\end{array}$ & 95\% CI \\
\hline Social Effect Strength, $s^{b}$ & & & 0.03 & $0.01,0.06$ \\
\hline Familiar ( $n=9$ groups) & 1 & & 0.05 & $0.03,0.09$ \\
Mixed ( $n=10$ groups) & 1 & & 0.04 & $0.02,0.07$ \\
Unfamiliar ( $n=9$ groups) & 1 & & & \\
\hline Individual-level Variables & & & Total Support & $-0.05,0.06$ \\
\hline Body Length (mm) & 1 & $29.27 \%$ & -0.05 & $-0.26,0.16$ \\
Block (B) & 1 & $32.34 \%$ & 0.28 & $0.08,0.48$ \\
Time since habituation & 1 & $94.86 \%$ & -0.10 & $-0.42,0.21$ \\
Patch Type (Control) & 1 & $43.66 \%$ & 0.003 & $-0.10,0.11$ \\
Patch Side (Left) & 1 & $26.45 \%$ & & \\
\hline
\end{tabular}

$a$ : Total support was based on Akaike weights calculated over the entire model set.

$b$ : Social effect parameter estimates and their $95 \% \mathrm{CI}$ are conditional on the top model from the additive OADA model subset that included the association networks. This model allowed social effects to differ between treatments-but not between patch types - and included an individual's time since last habituation as an individual-level variable. Confidence intervals were constructed using profile likelihood techniques and were adjusted to account for model selection uncertainty (Burnham \& Anderson, 2002). $c$ : Individual-level variable parameter estimates and unconditional standard errors were obtained via model-averaging over the additive OADA model subset that included the association networks. Unconditional standard errors were used to construct $95 \%$ confidence intervals. The baseline discovery rates for experimental block A, the real patch, and for patches on the right side of the arena were used as the overall baselinei.e., they equaled 0 . 
support; Table 3.2). There was strong evidence for no difference in social effect strength between real and control patches ( $82.1 \%$ support), suggesting information flow most likely resulted from untransmitted social effects. There was more support for a difference in social effect strength between treatments than against (63.9\% support).

The additive OADA variant that used the association networks received the greatest support (Table 3.1). Therefore, we used that model subset to obtain modelaveraged estimates and CIs (Table 3.3). All treatments appeared to exhibit social influences on patch discovery, as $95 \%$ confidence intervals for the social effect parameters did not overlap with 0 (Table 3.3). The social effect parameter is interpreted as the linear increase in patch discovery rate per unit of network connection to informed individuals relative to the average asocial rate of discovery (Hoppitt \& Laland, 2013). Social effects were strongest in mixed groups and weakest in familiar ones, with the difference estimated to be 0.02 ( $95 \% \mathrm{CI}: 0.01,0.05)$. As this confidence interval did not include 0 , it provided additional support for the treatment differences in social effect strength suggested by the summed Akaike weights (Table 3.2). There was less evidence for differences in social effect strength between mixed and unfamiliar groups-estimated difference 0.01 (95\% CI: 0, 0.04)—or between unfamiliar and familiar groupsestimated difference 0.01 (95\% CI: $0,0.03$ ) —as the confidence intervals for these estimated differences overlapped with 0 . The only individual-level variable that received strong support was the number of days since an individual's last habituation session ( $94.9 \%$ support). Individuals exhibited a difference in their baseline discovery rate of 0.28 (95\% CI: $0.08,0.48)$ on the log scale for every elapsed day relative to other individuals; for example, if 3 days had passed for individual A, whereas only 1 day had 
elapsed for individual $\mathrm{B}$, individual A would have a baseline discovery rate of $\exp [(3-1)$ $\times 0.28]=1.75$ times that of $\mathrm{B}$.

\section{Discussion}

The proportion of familiar individuals within guppy shoals did not influence the mean fission-fusion behavior exhibited by group members - that is, the probability of initiating or ending social contact or of switching between shoaling partners. This is surprising, given that previous studies have found that familiarity influences social decision-making and affects social network structure (e.g., Lachlan et al., 1998; Atton et al., 2014; Kohn et al., 2015). Individual-based models of collective motion have found that stronger social preferences between individuals - mimicking the effects of familiarity — can result in familiar individuals clustering together, forming distinct subgroups within the larger collective (Hemelrijk \& Kunz, 2005). Furthermore, several studies across a range of taxa have found that individuals are more likely to approach familiar group mates (Lachlan et al., 1998; Keller et al., 2011; Kohn et al., 2015) and associate more frequently with them (Atton et al., 2014).

Our results suggest that variation between groups in terms of the presence and distribution of social preferences may not necessarily translate into group-level differences in fission-fusion dynamics. This is likely to be particularly the case if strong social preferences are not concentrated within clusters of individuals. Models of collective motion have found that the effect of strong social ties on the size, cohesion, and membership of subgroups critically depends on the distribution of those ties within the overall population and that, in many ways, the dynamics of collective motion in populations with randomly distributed strong social ties greatly resembles those of 
populations lacking any strong ties at all (Bode et al., 2011b). The similarity in fissionfusion dynamics between our treatments could have consequences for social processes, such as the success of cooperative strategies (Santos et al., 2006b), in which outcomes are influenced by the frequency of social encounters and opportunities to switch between partners.

The lack of treatment effects on fission-fusion behavior might also be explained by several alternative possibilities. First, although familiarity can develop in as little as 12 days in guppies (Griffiths \& Magurran, 1997) and protocols similar to ours have been successfully used to promote familiarity in both wild and domestic guppies (e.g., Griffiths \& Magurran, 1997; Lachlan et al., 1998; Swaney et al., 2001; Granroth-Wilding \& Magurran, 2013), we cannot discount the possibility that our experimental protocol was ineffective in developing familiarity within groups. Another possibility is that because all individuals in our study were fed an identical diet, self-referential matching based on dietary cues allowed for some level of social recognition in all our groups, potentially masking treatment differences in fission-fusion behavior (Webster, Goldsmith, et al., 2007; Ward et al., 2009). It may also be that the expression of familiarity-based differences in shoaling depends on environmental conditions - for example, the level of predation risk. Groups of familiar fish exhibit greater shoaling cohesion in response to heightened predation risk or potentially threatening novel environments than do unfamiliar groups (Chivers et al., 1995; Granroth-Wilding \& Magurran, 2013). However, all fish in our study had experience within the arena prior to testing and all groups attempted to feed from the foraging patch, suggesting that the testing procedure was unlikely to have been perceived as especially threatening. Finally, 
early life experience can influence an individual's social behavior later in life (Chapman et al., 2008; Lindeyer et al., 2013). At the time females were selected for our experimental groups, the population size within each rearing tank varied to some extent (range: 17-24 individuals). Presumably, the sex ratio within rearing tanks varied as well. Although we find it unlikely that early life experience varied systematically between our treatments, it may still have contributed further variation to our results that obscured differences among treatments.

Randomization tests controlling for the frequency and duration of contact phases with nearest neighbors found that fish in both familiar and unfamiliar groups exhibited nonrandom nearest neighbor preferences, whereas individuals in mixed groups did not. Furthermore, nearest neighbor preferences, when present, were not expressed in the mean duration of contact with specific partners but in the frequency with which individuals were selected as a partner. This is consistent with the work done on wild guppies (Wilson et al., 2014, 2015), though it may be that more data are required to establish whether nearest neighbor preferences were expressed in terms of contact duration because contact phases of longer duration occurred less frequently. Additionally, single bouts of shoaling between two individuals often were broken up into several contact phases due to the fact that only the focal individual's nearest neighbor — rather than all nearby individuals—was identified at each time point.

Analysis of association networks based on contact phase frequency found that mixed groups were strongly assorted by shoaling tendency, such that individuals that spent much of their time shoaling were more likely to associate with one another, whereas unfamiliar groups exhibited tendencies toward assortment by activity level. 
There was no correlation between association strength and phenotypic similarity for any of the traits that we measured in familiar groups. Consistent between-individual variation in both attraction toward conspecifics and activity level has been observed across a range of taxa, including common lizards (Lacerta vivipara) (Cote \& Clobert, 2007), social spiders (Pruitt \& Goodnight, 2014), sticklebacks (Laskowski \& Bell, 2014), and guppies (Croft et al., 2009; Smith \& Blumstein, 2010). Such personality traits can be an important driver of group-level social structure and outcomes (Pike et al., 2008; Aplin et al., 2013; Farine, Montiglio, et al., 2015), and individuals assorting by behavioral type has been demonstrated in some species (Croft et al., 2009; Aplin et al., 2013). For example, male great tits (Parus major) were more likely to associate with other males that scored similarly on the proactive-reactive personality axis (Aplin et al., 2013), whereas wild guppies were assorted by their propensities to shoal with conspecifics and to engage in predator inspection (Croft et al., 2009). In the current study, it is possible that association patterns were influenced to a greater degree by group members' behavioral type in mixed and unfamiliar groups due to the absence or reduction of familiarity-based social influences. If this is the case, then as familiarity develops between group members, the relationship between an individual's behavioral type and their social behavior is predicted to shift.

One process by which such a shift could occur is through social niche specialization, which predicts that repeated interactions between group members drive increased between-individual variation and within-individual consistency in behavior in order to reduce social conflict (Bergmüller \& Taborsky, 2010; Montiglio et al., 2013). Direct support for social niche specialization has been found in social spiders (Laskowski 
\& Pruitt, 2014; Modlmeier, Laskowski, et al., 2014), though work on meerkats (Suricata suricatta) (Carter et al., 2014) and sticklebacks (Laskowski \& Bell, 2014) failed to detect such an effect. Social network analysis provides a framework in which to test the effects of social niche specialization on the consistency of social traits (Wilson et al., 2013). The relative consistency of an individual's network position may be ecologically relevant, as various network measures have been correlated with individual variation in social learning (Claidière et al., 2013) and reproductive success (McDonald, 2007). Social niche specialization might also be reflected in the distribution of social relationships within a group if consistent between-individual behavioral variation is associated with consistent differences in social preferences. We would then predict that groups composed of unfamiliar individuals would exhibit inconsistent social preferences and/or more evenly distributed network connections. The disruption of nearest neighbor preferences in our mixed groups, presumably due to the introduction of unfamiliar individuals, is consistent with such a process, though similar patterns were not observed in our unfamiliar groups. Future work examining the relationships between the development of familiarity, individual personality, and longitudinal changes in social network structure is likely to be highly informative (Montiglio et al., 2013; Wilson et al., 2013).

The order in which fish discovered a novel foraging site was predicted by their social networks. This result matches similar findings reported in a range of species, indicating that social networks can influence the diffusion of information through social groups (Claidière et al., 2013; Webster et al., 2013; Aplin et al., 2015). However, there was little support for social transmission of information in our study. Instead, it is likely that information diffusion occurred primarily due to untransmitted social effects. Social 
transmission occurs when the behavior of informed individuals - for example, feeding strikes, knowledgeable individuals acting as leaders - increases the probability of naïve individuals becoming informed (Hoppitt \& Laland, 2013). Conversely, closely associated individuals might acquire the same information through individual learning at similar times to one another due to their tendency to remain in close proximity - that is, an untransmitted social effect. This mechanism is consistent with learning via local enhancement, where individuals are attracted to a site due to the presence of other individuals and may thus be more likely to acquire information about that site (Thorpe, 1956; Hoppitt \& Laland, 2013).

In our study, social effect strength was equivalent when comparing diffusions regarding the real patch to those of the control. Although this does not eliminate the possibility of social transmission, it does suggest that if a social transmission effect is present, it is no stronger than the more parsimonious explanation of information flow occurring primarily via untransmitted social effects (Atton et al., 2012). Conversely, if social effect strength was greater for the real patch relative to the control, this would point to a role for social transmission in addition to any untransmitted social effects at work. However, the precise mechanism underlying social transmission would still need to be determined.

The strength of social effects on patch discovery differed between treatments, with mixed groups experiencing the fastest diffusion rates and familiar groups the slowest. Familiarity between shoal mates has been demonstrated to enhance the diffusion of foraging information in fish as a result of an increased tendency to follow familiar individuals (Lachlan et al., 1998; Swaney et al., 2001; Atton et al., 2014). However, our 
results point to an additional role for group composition in terms of familiarity in determining the speed of information flow through social groups. Group phenotypic composition has been shown to have wide-ranging effects on group-level outcomes (Farine, Montiglio, et al., 2015), including collective foraging success (Dyer et al., 2009; Aplin et al., 2014), group exploration (Hui \& Pinter-Wollman, 2014), and information flow (Morrell et al., 2008; Atton et al., 2014). Benefits of within-group behavioral diversity can arise through mechanisms such as negative frequency dependence (Maynard Smith, 1982) — that is, behavioral strategies receive lower payouts as they become more common — or social heterosis (Nonacs \& Kapheim, 2007), where the presence of alternative behavioral types within a group can be mutually beneficial. Although information is predicted to flow most readily between familiar fish, novel information may be more likely to originate from unfamiliar individuals if those individuals are also more likely to occupy different habitats or exhibit different diet preferences. Such an effect might underlie preferences for unfamiliar individuals observed in some contexts (Morrell et al., 2007; Galef \& Whiskin, 2008). Furthermore, the introduction of unfamiliar individuals into our mixed groups might have facilitated information diffusion throughout the network as a whole by promoting greater mixing and a more homogeneous social organization. Simulation studies of disease transmission using models of fission-fusion dynamics found that individuals became infected more rapidly in the absence of individual-specific social preferences (Wilson et al., 2014). Similarly, simulations of social learning over real-life primate networks have found that greater homogeneity in both structural patterns and association strength increases the rate at which information spreads through a group (Voelkl \& Noë, 2010). Another alternative 
involves the presence of dense, interconnected clusters of social individuals in mixed groups, resulting from strong assortment by shoaling tendency. Within such clusters, information would be predicted to flow especially rapidly. These clusters might also potentially act as information hubs for the rest of the network. Likewise, highly interconnected individuals within heterogeneously structured networks facilitate the diffusion of information or disease by bridging the gap between otherwise weakly connected individuals (e.g., Pinter-Wollman et al., 2011).

Increasing recognition is being given to the importance of group phenotypic composition in influencing individual fitness and driving group-level outcomes (Farine, Montiglio, et al., 2015; Kurvers et al., 2014). Our study adds to this literature by demonstrating that variation in the proportion of familiar individuals within a group can influence its social network structure and the speed at which information flows through it, even in the absence of differences in fission-fusion dynamics. An important next step is to determine the robustness of our results and the extent to which these patterns may act in natural populations. Future work could also examine shifts in network structure as familiarity between group members develops as well as the role of social niche specialization and individual personalities in shaping these processes (Montiglio et al., 2013; Wilson et al., 2013). Social networks mediate a wide swath of animal behavior, including mating, transmission dynamics, and cooperation (Hasenjager \& Dugatkin, 2015; Krause et al., 2015), as well as play an important role in shaping evolutionary outcomes (Kurvers et al., 2014). A better understanding of the links between group composition and the emergent structure and properties of social networks will prove highly beneficial to our understanding of behavioral ecology. 


\section{CHAPTER IV}

\section{GROUP PERSONALITY COMPOSITION SHAPES SHOALING DYNAMICS, NETWORK STRUCTURE, AND INFORMATION FLOW}

\section{Introduction}

The benefits of group-living, such as reduced predation risk and increased access to social information (Krause \& Ruxton, 2002; Ward \& Webster, 2016), must be balanced against its costs, including increased competition over resources (Metcalfe \& Thompson, 1995), a higher risk of infection (Keiser et al., 2016), and aggressive interactions with group members (Sih \& Watters, 2005; Schürch et al., 2010). This cost to benefit ratio varies according to phenotypic characteristics, such as age, sex, reproductive state, or hunger level, as well as with the size and phenotypic composition of a group (Krause \& Ruxton, 2002; Farine, Montiglio, et al., 2015). Members of phenotypically homogeneous groups can experience reduced predation risk (Landeau \& Terborgh, 1986; Rodgers et al., 2015) and lower costs of behavioral synchrony (Conradt \& Roper, 2000). Conversely, within-group phenotypic diversity can facilitate flexible group-level responses to environmental variation (Burns \& Dyer, 2008; Michelena et al., 2010) and can enhance group-level outcomes via synergistic interactions between phenotypic traits (Pruitt \& Riechert, 2011; Aplin et al., 2014). As such, whether or not grouping is beneficial varies between individuals, across groups, and over time. 
Personality—defined as consistent between-individual behavioral variation over time and across contexts-represents an important source of phenotypic variation (Réale et al., 2007; Stamps \& Groothuis, 2010). The willingness of an individual to accept greater risks in return for potentially greater rewards, i.e., boldness, is a well-studied facet of personality (Réale et al., 2007) that may reflect a general trade-off between life history strategies optimized for productivity versus those prioritizing survival (Smith \& Blumstein, 2008). As a result of its influence across a range of behavioral contexts, an individual's position on the bold-shy axis frequently shapes the nature and outcomes of its social interactions (Harcourt et al., 2009; Kurvers et al., 2010; Aplin et al., 2013). Shyness is generally associated with greater social attraction (Michelena et al., 2009; Aplin et al., 2014; Jolles et al., 2015), increased responsiveness towards group mates (van Oers et al., 2005; Nakayama et al., 2016), and a greater likelihood of acquiring and/or using social information (Kurvers et al., 2010). Conversely, bolder individuals often initiate and lead more collective movements (Harcourt et al., 2009; Kurvers et al., 2012; Nakayama et al., 2016), thereby driving group decision-making to align more closely with their personal preferences (though see McDonald et al., 2016).

Within-group variation in personality can also impact the fitness of group members and influence group-level outcomes (Farine, Montiglio, et al., 2015). In some contexts, collective outcomes result from the combined additive effects of group members' personalities (Hui \& Pinter-Wollman, 2014; Laskowski \& Bell, 2014), meaning that groups containing a higher proportion of shy individuals should behave more risk-aversely than groups composed predominately of bolder individuals. Increased personality diversity within a group can also enhance collective outcomes due to 
synergistic interactions between personality types (Burns \& Dyer, 2008; Pruitt \& Riechert, 2011; Modlmeier et al., 2012). As an example, groups that contain a mix of bold and shy individuals can experience enhanced foraging efficiency resulting from bolder individuals leading collective movements towards unexploited patches, while more risk-averse group members maintain a group's cohesion (Dyer et al., 2009; Aplin et al., 2014). Group dynamics can also be shaped disproportionately by the personality of one or a few 'keystone' individuals (Sih \& Watters, 2005; Modlmeier, Keiser, et al., 2014).

Social network analysis can provide insight into the mechanisms by which individual-level personality and group composition shape a number of social processes, such as collective decision-making, cooperation, and the transmission of information (Pinter-Wollman et al., 2014; Hasenjager \& Dugatkin, 2015; Krause et al., 2015). Network analysis quantifies a population as a collection of nodes (representing individuals) connected by ties indicating social relationships; the resulting pattern of connections defines the structure of a network (Croft et al., 2008). Increasing evidence suggests that individual-level personality often drives variation in network structure (Pike et al., 2008; Aplin et al., 2013; Snijders et al., 2014) and can affect phenotypic assortment within groups (Croft et al., 2009; Keiser et al., 2016). In general, bolder, more proactive individuals tend to associate with a greater number of conspecifics, but these associations tend to be weaker and more evenly distributed across a population than those of shyer, more reactive individuals (Pike et al., 2008; Croft et al., 2009; Aplin et al., 2013).

Social networks are often constructed by aggregating all of the interactions observed for a group over a certain period of time to create a static representation of 
social structure (Blonder et al., 2012; Pinter-Wollman et al., 2014). A weakness of such representations is that they provide little information on the underlying social dynamics which generated the observed structure (Blonder et al., 2012; Wilson et al., 2014). However, knowledge regarding the sequence, duration, and frequency of interactions can offer insight into the functioning of social processes that occur over timescales of seconds or minutes, such as cooperative interactions and information transmission (Croft et al., 2006; Rosenthal et al., 2015). Furthermore, similar network structures can arise from very different interaction sequences; for example, the weight of a network edge connecting two individuals does not indicate whether their interactions were clustered in time or occurred at more regularly spaced intervals. As such, examination of the underlying social dynamics could highlight important biological variation that would be overlooked by more coarse-grained approaches. A recently proposed method to address these weaknesses used Markov chains to model the fission-fusion decisions of individual group members (Wilson et al., 2014). Here, I used this method to investigate how group personality composition influenced the social dynamics from which network structures emerge.

Social interactions can facilitate the transmission of information between individuals, such as the location of foraging sites or the presence of a potential predator (Swaney et al., 2001; Ferrari et al., 2007; Aplin et al., 2012). Individual-level boldness and group personality composition are predicted to influence transmission processes for the following reasons: (i) personality variation can drive patterns of social connectivity (Croft et al., 2009; Aplin et al., 2013; Keiser et al., 2016); (ii) shyness is positively correlated with social responsiveness and social information use (van Oers et al., 2005; 
Kurvers et al., 2010; Jolles et al., 2015); and (iii) bolder individuals are more likely to engage in personal sampling of the environment and to initiate and/or lead collective movements (Dyer et al., 2009; Harcourt et al., 2009; Aplin et al., 2014). NBDA was developed to assess the likelihood that social transmission is involved in the spread of a novel behavioral trait (or novel information) and to examine how various factors shape the diffusion process (see Chapter I; Franz \& Nunn, 2009; Hoppitt \& Laland, 2013). Here, I used NBDA to test whether individual- and group-level personality variation influenced how rapidly and in what order fish solved a novel foraging task.

The Trinidadian guppy (Poecilia reticulata) possesses several qualities that make it an ideal model organism for the current study. Modern social network analyses have been used extensively to examine various aspects of guppy social biology, including cooperation during predator inspection (Croft et al., 2006), phenotypic assortment within shoals (Croft et al., 2004; Wilson et al., 2014), and social variation across environmental conditions (Kelley et al., 2011; Borner et al., 2015; Wilson et al., 2015). Guppies exhibit consistent between-individual variation along several personality axes, and this variation has been shown to influence group exploration (Brown \& Irving, 2014), social network structure (Croft et al., 2009), and foraging behavior (Dyer et al., 2009). Finally, the guppy has long served as a model system to examine questions related to social learning and information use (Dugatkin \& Godin, 1993; Swaney et al., 2001; Morrell et al., 2008).

Female guppies were exposed on repeated occasions to a simulated aerial predation event in order to assess their boldness. Groups of ten individuals were then constructed with one of the following compositions: (i) bold-dominated groups contained eight bold and two shy fish, (ii) mixed groups contained five guppies of each personality 
type, and (iii) shy-dominated groups contained two bold and eight shy fish. Each group was introduced into an arena where I recorded the shoaling decisions of group members. Markov chain models were used to examine whether fission-fusion dynamics were shaped by group personality composition and to infer the social network structure of each group. Next, I introduced a novel foraging task into the arena and recorded each individual's latency to solve the task. NBDA was used to examine the factors involved in the acquisition and diffusion of the task solution within the experimental groups.

I tested the following predictions. First, if boldness is negatively correlated with social attraction, then as the proportion of bold individuals within a group increases, individuals will exhibit less cohesive shoaling dynamics, characterized by a greater likelihood to cease shoaling and a lower probability of beginning to shoal. Furthermore, if individuals spend more time alone in bold-dominated groups, then the probability of changing nearest neighbors should decrease as a consequence of smaller group sizes. Second, I predicted that bold guppies would exhibit increased activity, spend less time shoaling, and be found in smaller groups than shy individuals, and that individual personality would have additive influences on the mean behavior of a group-e.g., bolddominated groups would be less social on average than shy-dominated groups. If shyness is positively correlated with social attraction, I would expect association strength would be highest between pairs of shy guppies, resulting in phenotypic assortment according to personality. Conversely, if boldness is correlated with leadership tendencies, such that shy individuals tend to follow bold leaders, this could result in phenotypic disassortment. With regards to solving the novel foraging task, I predicted that bold individuals would be faster to initially acquire the solution (e.g., as a result of weaker neophobic 
responses), while shy individuals would rely on social information to solve the task to a greater extent than bold individuals. Likewise, I predicted that bold-dominated and mixed groups would initially acquire the solution more rapidly than shy-dominated groups, while social transmission of the solution would occur more rapidly in mixed and shydominated groups relative to bold-dominated ones.

\section{Methods}

\subsection{Study Subjects and Rearing Conditions}

This study was conducted from September 2015 to May 2016 using lab-reared descendants of guppies collected in 2003 from the Quaré River, Trinidad. Juvenile guppies ( $\leq 7 \mathrm{~mm}$ in length) were collected en masse from four 208-L stock tanks-each of which contained several hundred fish — and were randomly assigned to one of 48 rearing tanks (37.9-L) in which they were raised to maturity. Rearing tanks contained 3-7 adult females and no more than 10 juveniles at any time. Males were removed to the stock tanks as they matured. Any females that were not raised within the rearing tanks were not used to construct the experimental groups. Rearing tanks were periodically restocked with juveniles drawn from the stock tanks as described above. Rearing tanks were visually and chemically isolated from one another, filtered, and maintained at 26-27 ${ }^{\circ} \mathrm{C}$ on a 12-h:12-h light-dark cycle. Each contained a layer of gravel $2 \mathrm{~cm}$ deep and artificial plants for shelter. Fish were fed Cobalt Aquatics® Tropical Flake Food daily and supplemented every other day with live Artemia nauplii.

\subsection{Boldness Assay}

Each experimental trial took place over five days. Prior to commencing the main study, a pilot study was conducted to determine whether an individual's boldness scores 
were consistent over this time frame. For the pilot study, boldness was assessed for 30 adult female guppies as described below, save that the second boldness assay for these individuals occurred four days after their first assay (rather than on the day after). For the main study, on the first day of an experimental trial, 32 non-gravid, size-matched females originating from separate rearing tanks were collected between 13:00 and 16:00. Females were each placed in separate opaque plastic containers filled with $500 \mathrm{~mL}$ of treated tap water. This allowed for individual identification during boldness assays without having to individually mark each female. Only females were used in this study as they tend to be more food-motivated than males (Reader \& Laland, 2000; Magurran, 2005) and sexbased variation in risk-taking propensities have been demonstrated in the guppy (Harris et al., 2010).

On the day following collection, females were exposed to a simulated aerial predation event in order to assess their boldness; the design was adapted from Chapman et al. (2010). Boldness assays took place in a 37.9-L tank filled with treated tap water to a depth of $5 \mathrm{~cm}$ (Figure 4.1). The tank walls were covered by opaque plastic and a $60 \mathrm{~W}$ incandescent bulb was suspended $95 \mathrm{~cm}$ above the tank. A monofilament line passed above the tank lengthwise on a $24^{\circ}$ angle and was attached at both ends to an exterior metal frame. The monofilament line was threaded through a tube attached to the back of a $17 \mathrm{~cm} \times 10.5 \mathrm{~cm}$ piece of cardboard, which hung suspended at the highest point. Black cloth was hung around the metal frame to further minimize disturbance from external visual stimuli. Assays were recorded with a Canon Vixia HG21 digital camcorder suspended above the tank. Two such apparatuses were constructed, allowing the boldness of two individuals to be assessed simultaneously. 
Boldness assays took place between 8:00 and 13:00. A female was placed alone in the tank and allowed to acclimate for eight minutes. Immediately following acclimation, the cardboard was released and travelled rapidly down the monofilament line (Figure 4.1). As it passed below the light, this caused a shadow to sweep across the tank. All guppies exhibited a startle response to this event that involved erratic dashes prior to freezing in place. Boldness was quantified as the latency to resume movement after freezing, defined as moving at least one body length from where the individual had frozen. Individuals that did not move for three minutes were given a maximum score of 180 seconds. Afterwards, individuals were returned to their holding container and fed two freeze-dried bloodworms. The water within each tank was replaced completely following every other boldness assay. On the following day, individuals' boldness was assessed again. Testing apparatus and order of testing were counterbalanced, such that each individual was assayed once in each apparatus and was assayed as both the first and second fish within an apparatus prior to a water change. Following the second assay, females were returned again to their holding containers and fed two freeze-dried bloodworms.

\subsection{Testing Procedure}

On the day following the second boldness assay, experimental groups were constructed. Individuals that had a mean latency to resume movement of less than 40 seconds were labeled as bold, while individuals with a mean latency to resume movement of greater than 60 seconds were labeled as shy. Additionally, no single measure of latency to resume movement was greater than 50 seconds for bold individuals, nor less than 50 seconds for shy individuals. Subjects that did not meet these criteria were 


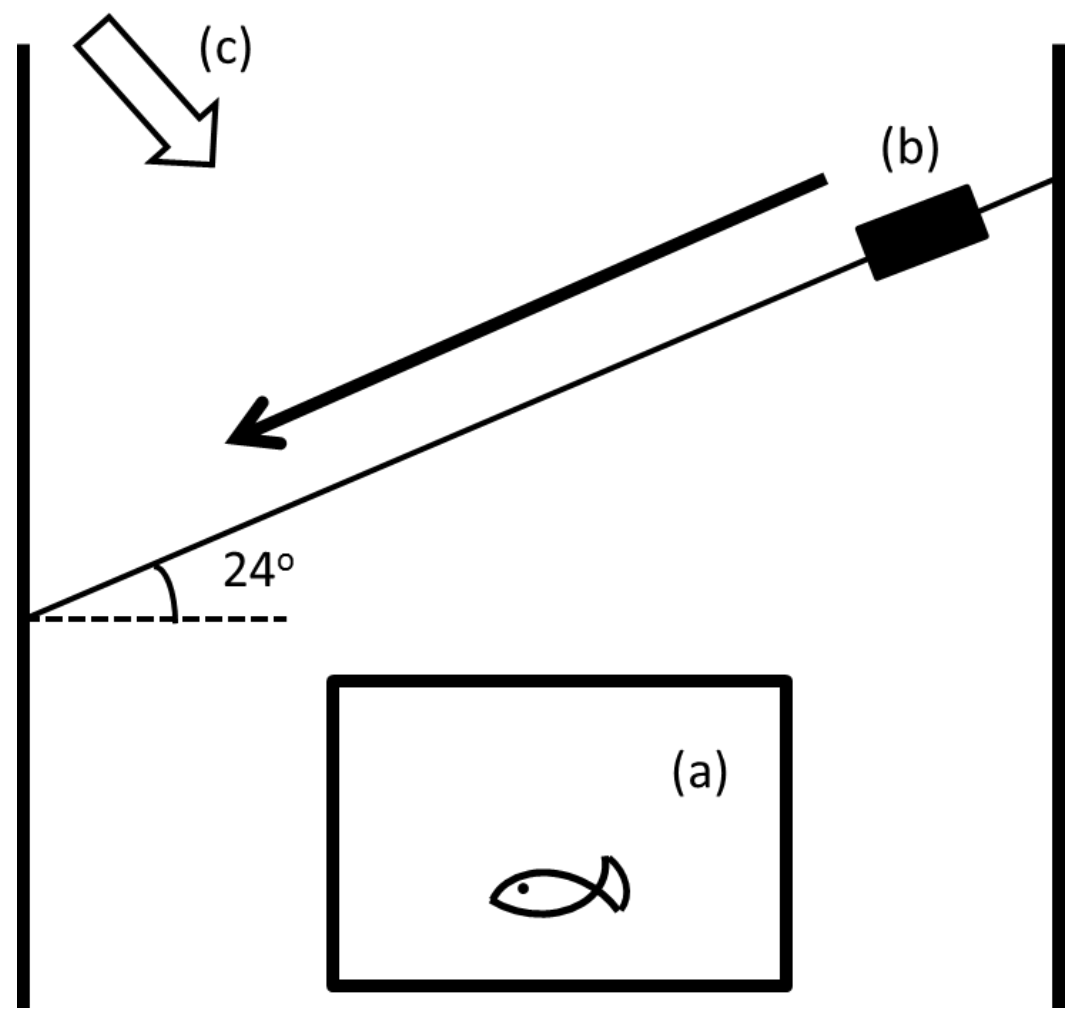

Figure 4.1 Boldness assay setup. A female guppy is placed alone in a 37.9-L tank (a). Following acclimation, cardboard suspended from a monofilament line (b) is released. As the cardboard travels down the line, it passes beneath a light source (c). This causes a shadow to sweep across the tank, thereby eliciting a startle response in the focal individual. An individual's latency to resume movement following this response was used as a measure of its boldness. 
returned to the stock tanks and were not used further in this study. While these cutoffs are arbitrary, the rationale behind them was to identify sets of individuals in which those labeled as shy consistently differed in their behavior relative to those labeled as bold. Within the experimental groups, the mean $( \pm \mathrm{SD})$ boldness score was $18.5 \pm 8.9$ seconds for bold individuals and $140.1 \pm 31.3$ seconds for shy individuals.

An experimental group was then constructed with one of three behavioral compositions corresponding to the treatments: (i) bold-dominated groups ( $n=12$ groups) were composed of eight bold fish and two shy ones, (ii) mixed groups ( $n=12$ groups) were composed of five fish of each personality type, and (iii) shy-dominated groups ( $n=$ 12 groups) were composed of two bold fish and eight shy ones. Within a group, individuals were size-matched to within $3 \mathrm{~mm}$ standard length. This was done in order to more effectively isolate the influence of boldness on shoaling decisions by minimizing the impact of size-based shoaling preferences (Krause \& Ruxton, 2002; Wilson et al., 2014). The mean ( \pm SD) standard length of individuals in this study was $1.79 \pm 0.11 \mathrm{~cm}$. A set of 32 fish occasionally yielded enough individuals that met the bold/shy criteria to allow for construction of two experimental groups. Overall, boldness assays were conducted on 27 sets of 32 fish for a total of 853 individuals tested (11 mortalities occurred in the holding containers).

To permit individual identification within an experimental group, individuals were anesthetized with MS-222 buffered with sodium bicarbonate and injected with a combination of two colored elastomer tags (Northwest Marine Technologies, Inc.; Shaw Island, WA, U.S.A.). This method has been used extensively with guppies (Croft et al., 2006; Morrell et al., 2008; Hasenjager \& Dugatkin, 2017) and does not appear to 
influence shoaling decisions (Croft et al., 2004). Following marking, groups were allowed to recover for at least one hour in treated, aerated tap water. No mortalities occurred as a result of this procedure. Following the recovery period, groups were introduced into the testing arena.

The testing arena was a black rectangular tank $(84$ x $51 \mathrm{~cm})$ containing black gravel substrate and treated tap water at a depth of $7 \mathrm{~cm}$ (Figure 4.2). A heater recessed into the substrate maintained water temperature at $26-27^{\circ} \mathrm{C}$. Seventeen black plastic partitions $10 \mathrm{~cm}^{2}$ in area were set up perpendicular to one another in order to break up lines of sight between fish within the arena. Overhead illumination was provided by four 23 W compact fluorescent lights. Groups were filmed by a Canon Vixia HG21 digital camcorder suspended $1.2 \mathrm{~m}$ above the arena. Black cloth was hung around the arena to minimize disturbance from external visual cues in the room. At least one hour following introduction into the arena, groups were fed flake food ad libitum; after one hour, uneaten food was removed from the arena. Groups were then allowed to acclimate within the testing arena overnight. Two such arenas were set up to permit simultaneous testing of two groups. Six groups from each treatment were tested within each arena.

Testing began at 13:00 on the day following group construction. Groups were filmed for 120 minutes to collect shoaling association data following the methods described in Wilson et al. (2014). Focal individuals were randomly selected and observed continuously for 4 minutes. Every ten seconds, I recorded whether that individual was alone - that is, no group mates were within four body lengths — or whether it was shoaling. If the latter, the identity of its nearest neighbor was recorded as measured from the center of their heads. Additionally, its group size was calculated as the number of 


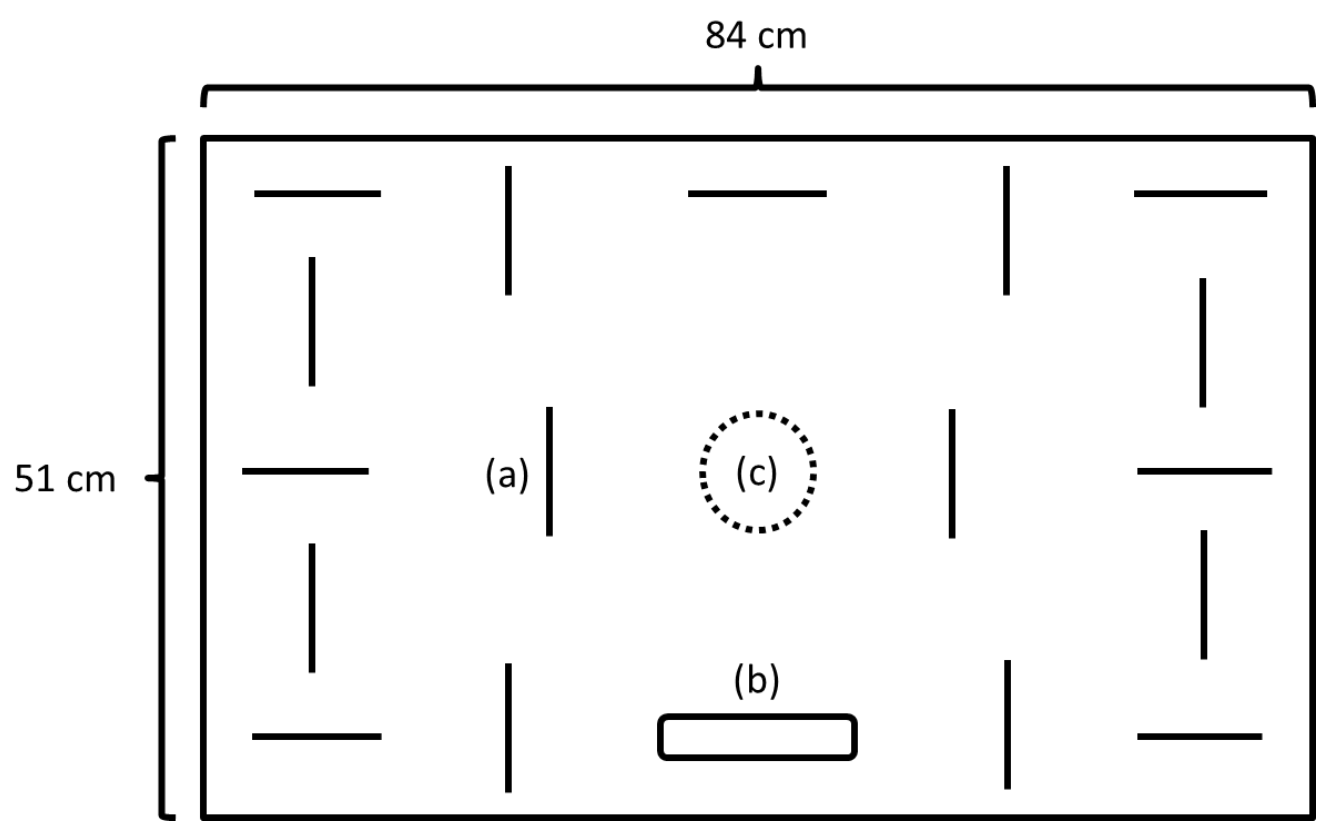

Figure 4.2 Test arena setup. The arena measured 84 x $51 \mathrm{~cm}$. It contained black gravel substrate and treated tap water $7 \mathrm{~cm}$ deep. Seventeen $10 \mathrm{~cm}^{2}$ partitions (a) were used to create a structurally complex space, while a heater (b) maintained water temperature at 26-27 ${ }^{\circ} \mathrm{C}$. After recording groups for 120 minutes to collect shoaling behavior data, a novel foraging device (c) was introduced into the arena. For those individuals that successfully used the device, their latency to first enter it was recorded. 
individuals who were within four body lengths, as well as the number of additional individuals who were within four body lengths of those group mates. I also recorded activity based on whether the focal individual had moved $\geq 10 \mathrm{~cm}$ since its prior observation. After four minutes, a new focal fish was randomly selected until all group members had been observed in this way. This process was repeated twice more to provide 72 observations per individual. Where possible, I avoided selecting focal fish who had been the nearest neighbor of the prior focal individual within the previous 60 seconds.

After 120 minutes, a novel foraging device was gently introduced into the arena. The device was a white PVC tube $8.6 \mathrm{~cm}$ tall and $8.9 \mathrm{~cm}$ in diameter with a grey plastic base and a hole ( $2 \mathrm{~cm}$ diameter) drilled into the lower half to permit entry. The cylinder was stocked with 32 freeze-dried bloodworms that floated on the water's surface. These food items were not visible to individuals from outside the device. Groups were filmed for an additional 20 minutes; for those individuals that successfully used the device, I recorded their latency to first enter it. Following testing, groups were returned to the stock tanks and were not used further in this study. One bold individual died in one of the bold-dominated groups prior to testing; thus, one group of nine fish was included in the analyses.

All experimental procedures, as well as animal care and maintenance protocols, were approved prior to the study's commencement by the University of Louisville's Institutional Animal Care and Use Committee (IACUC \#13020). 


\subsection{Statistical Analyses}

\subsubsection{Repeatability of Boldness}

The repeatability of individuals' boldness scores in both the pilot study and full study were estimated using the rptR package (Nakagawa \& Schielzeth, 2010) in R version 3.3.1 (R Core Team, 2016). A linear mixed effects model was fitted using restricted maximum likelihood with boldness scores as a dependent variable and individual identity as a random effect. Variance estimates were extracted from the individual identity random effect and the random error term and used to calculate repeatability as described in Nakagawa and Schielzeth (2010). The standard error and 95\% confidence intervals for repeatability were obtained via parametric bootstrapping with $10^{4}$ simulated datasets generated. Significance of the individual identity random effect was assessed using a likelihood ratio test (Bolker et al., 2009; Nakagawa \& Schielzeth, 2010). The repeatability estimate for the full study included all females whose boldness was assayed, regardless of whether or not they were used to construct the experimental groups. The relationship between an individual's mean boldness score and their body length was examined via Pearson's product-moment correlation coefficient for those individuals included in the experimental groups. As a near-significant correlation was detected, body length was included in the (generalized) linear mixed models and network-based diffusion analysis (see below).

\subsubsection{Markov Chain Fission-Fusion Models}

To characterize the shoaling dynamics within each treatment, I used the model of fission-fusion behavior originally developed by Wilson et al. (2014). The shoaling behavior of a focal individual is described by a sequence of behavioral states, where 
swimming alone is denoted by $a$ and shoaling is denoted by $s_{g}$, where $g$ is one of $k$ potential nearest neighbors. If $a$ and $s_{1} \ldots s_{k}$ are regarded as states of a first-order Markov chain, then the probability of transitioning between states is determined solely by an individual's current state. The first-order model can be characterized by the probabilities of an individual switching from being alone to shoaling $[p(a, s)]$, of ceasing to shoal $[p(s$, a)], and of switching between nearest neighbors while continuing to shoal [ $p($ switch)] (Figure 1.4b). As some behavioral states were not observed with sufficient frequency to permit accurate estimation of individual-specific transition probabilities, data were pooled across all focal follows within each group. Transition probabilities were then estimated for each group as simple proportions from the observed sequences of shoaling behavior. Weighted mean transition probabilities were calculated for each treatment, where weighting was based on the relative frequency of opportunities for state transitions within a group.

Model goodness-of-fit was assessed by comparing the observed distributions of three variables - the durations of social contact, of contact with a particular nearest neighbor, and of being alone - with simulated distributions of those variables generated using the weighted mean transition probabilities estimated for each treatment. Each simulated run maintained the observed starting state and four-minute observation time for each focal follow; $10^{4}$ simulations were conducted for each treatment. These comparisons revealed that first-order Markov chain models provided a poor fit to the observed data, in that many observed values fell outside the $2.5 \%$ and $97.5 \%$ percentiles generated by the simulations. Therefore, I constructed second-order Markov chain models for each 
treatment in which the probability of transitioning between states is determined jointly by an individual's state at times $t$ and $t-1$ (Figure 4.3).

The second-order model is characterized by the probabilities: (i) of ceasing to shoal after having been alone at time $t-1$ and shoaling at time $t[p(a-s, a)]$; (ii) of ceasing to shoal after having been shoaling with the same nearest neighbor for two consecutive time points $\left[p\left(s-s \_s a m e, a\right)\right]$; (iii) of ceasing to shoal after having been shoaling with two different nearest neighbors at times $t-1$ and $t\left[p\left(s-s_{-}\right.\right.$diff, $\left.\left.a\right)\right]$; (iv) of starting to shoal after having been shoaling at time $t-1$ and alone at time $t[p(s-a, s)] ;(\mathrm{v})$ of starting to shoal after having been alone for two consecutive time points $[p(a-a, s)]$; (vi) of changing nearest neighbors after having been alone at time $t-1$ and shoaling at time $t[p(a-s$, switch)]; (vii) of changing nearest neighbors after having been shoaling with the same nearest neighbor for two consecutive time points [ $\left.p\left(s-s \_s a m e, s w i t c h\right)\right]$; and (viii) of changing nearest neighbors after having been shoaling with two different nearest neighbors at times $t-1$ and $t\left[p\left(s-s \_\right.\right.$diff, switch $\left.)\right]$.

As above, transition probabilities were calculated for each group using data pooled across all focal follows, and weighted mean transition probabilities were calculated for each treatment. Goodness-of-fit was assessed as described above, with each simulated run now maintaining the initial two states observed for each focal follow. The second-order model generated distributions of the durations of social contact, of being alone, and of shoaling with a particular nearest neighbor that provided a good match to the observed data (see Results). Nonparametric bootstrapping-generating $10^{4}$ simulated datasets - was used to obtain $95 \%$ confidence intervals for the weighted mean transition probabilities using the boot package (Davison \& Hinkley, 1997; Canty \& 


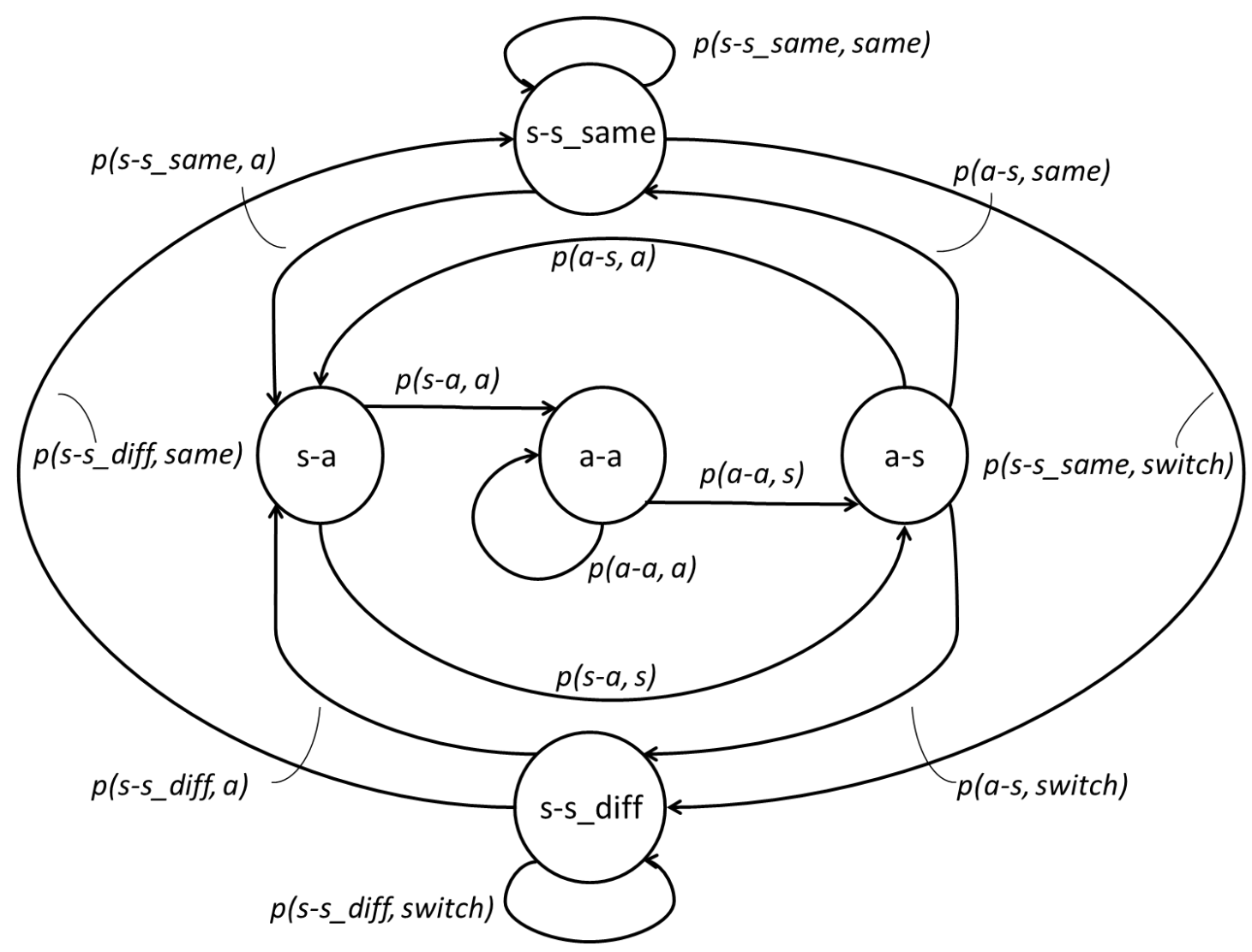

Figure 4.3 Markov chain model of shoaling behavior. In a second-order Markov chain, the probability of transitioning between states is determined jointly by an individual's state at times $t-1$ and $t$; these joint states are shown within the circles. At times $t-1$ and $t$, an individual could be alone and then shoaling $(a-s)$, shoaling and then alone $(s-a)$, alone at both time points $(a-a)$, shoaling with the same nearest neighbor at both time points $\left(s-s \_s a m e\right)$, or shoaling with a different nearest neighbor at each time point $(s$ $s \_$diff). At time $t+1$, an individual can be alone $(a)$, can begin to shoal $(s)$, can remain with the same nearest neighbor from time $t$ (same), or can switch between nearest neighbors from time $t$ to $t+1$ (switch). 
Ripley, 2016) in R version 3.3.1 (R Core Team, 2016).

\subsubsection{Nearest Neighbor Preferences}

To test whether fish expressed non-random nearest neighbor preferences, association strength was quantified between each pair in terms of: (i) the average duration of a contact phase between them, and (ii) their total number of shared contact phases. A contact phase between two individuals was defined as one or more consecutive observations in which one of the individuals was recorded as being the nearest neighbor of the other. I then employed randomization tests where the observed number of contact phases and their durations were maintained for each individual, but nearest neighbor identities were randomized prior to recalculating association strengths using both methods described above. This procedure was carried out $10^{4}$ times per group. The test statistic was the sum of squares of the association strength across all dyads. If the observed test statistic was in the top $5 \%$ of the distribution generated by the randomization procedure, this was used as evidence that focal fish were expressing individual-specific nearest neighbor preferences. Combined $P$ values for a treatment were obtained using Fisher's omnibus procedure (Haccou \& Meelis, 1994).

\subsection{4 (G)LMM Analysis of Individual Activity Levels and Shoaling Tendencies}

The influence of individual- and group-level personality variation on an individual's activity level, the amount of time individuals spent alone, and their mean group size when shoaling was examined using (generalized) linear mixed effects models [(G)LMMs]. Group composition, individual personality, and their interaction were treated as fixed effects, while group identity was included as a random effect. Body length was included as a covariate and standardized to zero mean and unit variance. An 
individual's activity level and overall time spent alone were counts of the number of observations in which an individual was active or alone respectively; due to significant overdispersion, these variables were analyzed using negative binomial GLMMs. An individual's mean group size when shoaling was analyzed with a LMM. As one bolddominated group only contained nine individuals, each individual's mean group size was divided by the maximum group size possible within their group. Statistical significance was assessed using likelihood ratio tests. For the analysis of mean group size, maximum likelihood estimation was used when conducting likelihood ratio tests, while restricted maximum likelihood was used for parameter estimation (Bolker et al., 2009; Zuur et al., 2009). GLMMs and LMMs were fitted using the glmmADMB and lme 4 packages respectively (Fournier et al., 2012; Skaug et al., 2014; Bates et al., 2015) in R version

\subsection{1 (R Core Team, 2016).}

\subsubsection{Phenotypic Assortment}

Using weighted association networks, where edge weights equaled the total number of shared contact phases between each pair (see Results), I examined whether groups exhibited assortment according to personality. Assortativity coefficients for weighted social networks $\left(r^{w}\right)$ were obtained using the assortnet package (Farine, 2014) in $\mathrm{R}$ version 3.3.1 (R Core Team, 2016); $r^{w}$ measures the extent to which associations occur between phenotypically similar or dissimilar individuals. When two phenotypic classes are present—e.g., bold and shy $-r^{w}$ ranges from fully assorted at 1 , meaning individuals only associate with others sharing their phenotypic class, to fully disassorted at -1 . 
Assortativity coefficients and standard errors were obtained for each group's association network and then averaged across the networks within a treatment. To assess whether mean $r^{w}$ for a treatment significantly differed from null expectations, $10^{4}$ randomized networks were constructed for each group by randomizing nearest neighbor identities while maintaining the observed number of contact phases. Within each treatment, mean $r^{w}$ was calculated for each set of randomized networks to generate a distribution of expected values. If observed mean $r^{w}$ was in the lower or upper $2.5 \%$ of the distribution of the randomized mean $r^{w}$, s, this was taken as evidence of a statistically significant pattern of (dis)assortment.

\subsubsection{Network-Based Diffusion Analysis (NBDA)}

To examine the rates and patterns of information diffusion within the experimental groups, I used the time-of-acquisition diffusion analysis (TADA) variant of NBDA (Franz \& Nunn, 2009; Hoppitt \& Laland, 2013). NBDA infers social transmission if the rate at which naïve individuals acquire novel information —or adopt a novel behavioral trait—increases with the strength of their connectedness to informed individuals within their group. An individual's latency to first enter the foraging device was used to indicate when that individual became informed regarding the location of food and how to access it. A general form of the NBDA model, extended to consider diffusions in separate groups, is given by:

$$
\lambda_{i k}(t)=\lambda_{0}(t)\left(1-z_{i k}(t)\right)\left(s \sum_{j=1}^{N_{k}} a_{i j k} z_{j k}(t)+1\right)
$$

in which $\lambda_{i k}(t)$ is the rate at which individual $i$ in group $k$ acquires the task solution at time $t, \lambda_{0}$ is the baseline (asocial) rate of acquisition, $z_{i k}(t)$ is the informational status of 
individual $i$ in group $k$ at time $t$ where $z=1$ is informed and $z=0$ is naïve, $s$ is the fitted social transmission parameter, and $a_{i j k}$ is the association strength between individuals $i$ and $j$ in group $k$ (see Hoppitt \& Laland, 2013 for additional details). The rate of social transmission from an informed to a naïve individual is assumed to be linearly proportional to the strength of network connection between them, $a_{i j}$, and scaled by $s$.

While a positive result for $s$ suggests that there are social influences on how rapidly an individual acquires information, it does not necessarily indicate social transmission when the behavioral trait of interest involves a spatial element, such as when discovering the location of a foraging patch. Social transmission occurs when the behavior of informed individuals positively influences the rate at which naïve individuals acquire the trait (Hoppitt \& Laland, 2013). Conversely, untransmitted social effects can occur when individuals acquire the same information at similar times simply as a result of their tendency to be found in close spatiotemporal proximity (Atton et al., 2012; Webster et al., 2013); under such a scenario, whether or not an individual is informed is irrelevant to that individual's influence on the rate at which its associate acquires the trait. To avoid counting such simultaneous solves of the foraging task as instances of information transfer, information was not permitted to transmit between individuals that acquired the task solution within 10 seconds of one another; similar approaches have been used in foraging bird flocks (Aplin et al., 2012; Farine, Aplin, et al., 2015). Ten seconds is more than sufficient time for a guppy to travel the length of the arena. Furthermore, social transmission can occur over shorter timescales (Strandburg-Peshkin et al., 2013; Ioannou et al., 2015), meaning the estimates of its effects in this study are potentially conservative. 
I examined the explanatory power of four network types representing potential transmission pathways: (i) association networks, where the association strength between each pair equaled their total number of shared contact phases (Figure 4.4a); (ii) homogeneous networks, where the association strength between each pair was set to 1 (Figure 4.4b); (iii) bold-leader networks, in which informed bold individuals transmitted their information at double the rate of informed shy individuals (Figure 4.4c); and (iv) shy-social-learner networks, in which information transmitted to shy individuals twice as fast as it did to bold ones (Figure 4.4d). Inclusion of the association networks tested whether social transmission rates were proportional to the association strength between individuals, while inclusion of the homogeneous networks tested whether social transmission operated independently of the social networks. The bold-leader networks tested whether bold individuals were more effective demonstrators-e.g., if informed bold individuals were especially likely to lead other group members to the food source (Aplin et al., 2014; McDonald et al., 2016). Finally, the shy-social-learner networks tested whether shy individuals were faster than bold individuals to acquire and/or use social information (Kurvers et al., 2010).

In addition to comparing the above network variants, the analysis considered two social transmission parameterizations: (i) models that permitted the strength of social transmission to vary based on group personality composition, and (ii) models that assumed social transmission strength was equal across all experimental groups. Individual-level variables were included in the analysis to assess their influence on an individual's baseline solving rate; these included: individuals' personality type, body length, and the personality composition of their group. Body length was standardized to 
(a)
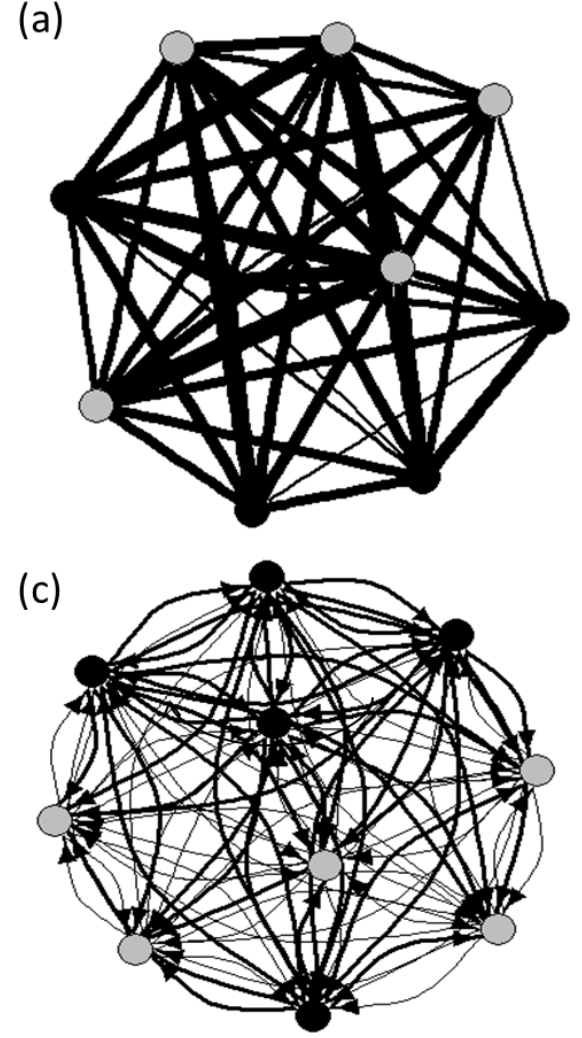

(b)

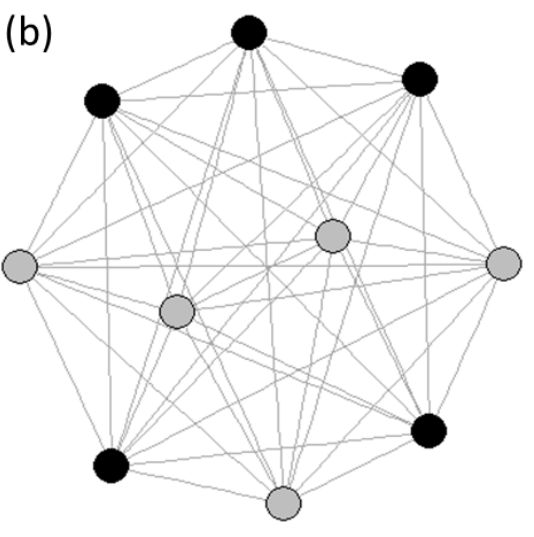

(d)

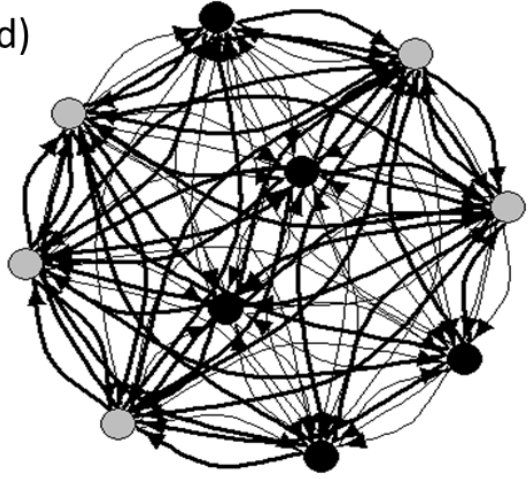

Figure 4.4 (a) An association network, in which network connections are undirected and weighted by the total number of shared contact phases between a pair of individuals. (b) A homogeneous network, in which network connections are undirected and all are of equal strength. (c) A bold-leader network, in which network connections are directed and those originating from bold individuals — depicted as black nodes — are weighted twice as heavily as connections originating from shy individuals-depicted as grey nodes. (d) A shy-social-learner network, in which network connections are directed and those directed towards shy individuals are weighted twice as heavily as connections directed towards bold individuals. 
zero mean and unit variance. Both additive and multiplicative TADA variants were considered (Hoppitt, Boogert et al., 2010). In the additive variant, individual-level variables affect only an individual's baseline solving rate-i.e., how rapidly does an individual acquire information on its own. In the multiplicative variant, these variables also affect social transmission, such that if individual A has a baseline solving rate twice that of individual B, individual A's total solving rate will remain twice that of B's as long as both are equally connected to informed individuals. Finally, models that allowed the baseline solving rate to systematically increase (or decrease) over time were compared with those that constrained it to be constant.

I adopted an information-theoretic approach for this analysis (Burnham \& Anderson, 2002). Models were fitted for every combination of network type, social transmission parameterization, individual-level variable(s), TADA variant, and baseline rate function. The model set further included models in which only individual learning was permitted $(s=0)$. Akaike weights based on AICc were obtained for each fitted model. Two models accounted for $>99.99 \%$ of the total Akaike weights. The top model accounted for $68 \%$ of the total Akaike weights, while the second-ranked model accounted for $32 \%$. The only difference between these two models was that the top model included a baseline solving rate that increased over time, while the second-ranked model fitted a constant baseline solving rate. As such, only the results of the top-ranked model are presented and used to draw conclusions.

Confidence intervals for parameter estimates were computed using profile likelihood techniques, while standard errors were obtained using a numerical estimate of the Hessian matrix via the optim function in R version 3.3.1 (Morgan, 2009; R Core 
Team, 2016). Finally, the estimated probability that individual $i$ solved the foraging task via social transmission is given by:

$$
s \sum_{j=1}^{N} a_{i j} z_{j}\left(t_{i}\right) /\left(s \sum_{j=1}^{N} a_{i j} z_{j}\left(t_{i}\right)+1\right)
$$

where $t_{i}$ is the time at which $i$ solved the task. This value was averaged across all individuals who solved the task within each treatment, as well as computed separately for each personality type within a treatment. NBDA was conducted in $\mathrm{R}$ version 3.3.1 (R Core Team, 2016) using code (version 1.2.11) freely available at http://lalandlab.standrews.ac.uk/freeware.

\section{Results}

\subsection{Repeatability of Boldness}

Boldness scores remained consistent over five days during the pilot study ( $n=30$, $\mathrm{R}=0.56 \pm 0.13 \mathrm{SE}, 95 \% \mathrm{CI}: 0.26-0.76, P<0.001)$ and across consecutive days during the main study $(n=853, \mathrm{R}=0.35 \pm 0.03 \mathrm{SE}, 95 \% \mathrm{CI}=0.29-0.41, P<0.001)$. For those individuals from which the experimental groups were composed, there was a nearsignificant positive correlation between individual body length and mean boldness score (2-tailed test: $\left.n=359, \mathrm{r}^{2}=0.10, P=0.061\right)$. As such, body length was included as a factor in the (G)LMMs and TADA (see below).

\subsection{Markov Chain Fission-Fusion Models}

Comparison of the observed distributions of the durations of social contact, of contact with a particular nearest neighbor, and of being alone with simulated distributions generated from the weighted mean transition probabilities estimated for each treatment indicated that first-order Markov chain models provided a poor fit to the observed data. 
Modeling shoaling behavior using second-order Markov chains, in which transition probabilities are defined jointly by behavior at times $t-1$ and $t$, better reflected the observed fission-fusion dynamics-i.e., the majority of observed values fell within the $2.5 \%$ and $97.5 \%$ percentiles generated by the model (Figure 4.5 ). This suggests that shoaling decisions were influenced by both an individual's current and recent behavior. While some deviations from the models' predictions were present, the predicted percentile ranges were conservative in that the simulated distributions did not account for uncertainty in the transition probability estimates.

Weighted mean transition probabilities did not differ greatly between treatments in terms of the probabilities of moving from an asocial to a shoaling state or vice versa, suggesting that group personality composition did not influence these aspects of guppy shoaling behavior (Figure 4.6 a-e). In contrast, individuals in bold-dominated groups were less likely to switch between nearest neighbors from time $t$ to $t+1$ than were individuals in shy-dominated groups (Figure $4.6 \mathrm{f}-\mathrm{h}$ ). This was particularly evident for $p\left(s-s \_s a m e\right.$, switch) and $p\left(s-s \_\right.$diff, switch) (Figure $\left.4.6 \mathrm{~g}, \mathrm{~h}\right)$; mixed groups exhibited intermediate values for these transition probabilities.

\subsection{Nearest Neighbor Preferences}

Nearest neighbor preferences were not expressed in the average duration of contact between two individuals (Omnibus tests: bold-dominated: $\mathrm{X}^{2}=7.78, \mathrm{df}=24, P>$ 0.999; mixed: $\mathrm{X}^{2}=24.86, \mathrm{df}=24, P=0.413$; shy-dominated: $\mathrm{X}^{2}=29.40, \mathrm{df}=24, P=$ 0.206). Rather, when association strength was calculated as the number of shared contact phases between each pair, non-random nearest neighbor preferences were observed in all treatments (Omnibus tests: bold-dominated: $\mathrm{X}^{2}=189.26, \mathrm{df}=24, P<0.001$; mixed: $\mathrm{X}^{2}=$ 

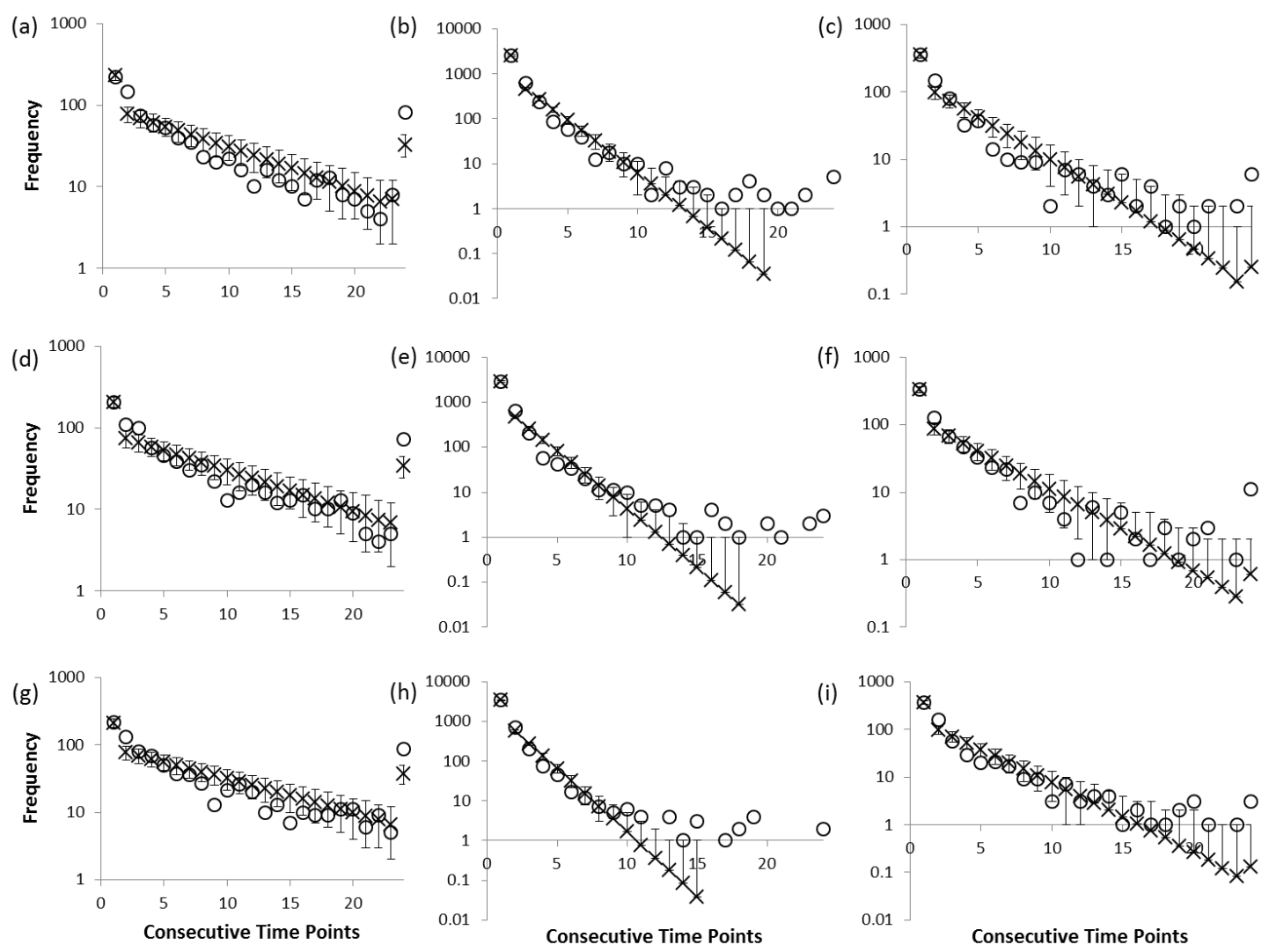

Figure 4.5 Frequency distributions of (a) the durations of continuous social contact, (b) the durations of contact with a particular nearest neighbor, and (c) the durations of time spent alone for bold-dominated groups ( $n=12$ groups). Observed data are depicted as circles. Using weighted mean transition probabilities, $10^{4}$ simulations of the second-order Markov chain model were generated. The mean values ( $x$ 's) and the $2.5 \%$ and $97.5 \%$ percentiles obtained from these simulations are depicted. Values of 0 cannot be displayed on a logarithmic plot and are omitted. For each simulated run, the initial two states observed for each focal individual and the four-minute observation time per focal follow were maintained. The corresponding data for mixed groups ( $n=12$ groups), (d)-(f), and for shy-dominated groups ( $n=12$ groups), (g)-(i), are also depicted. 

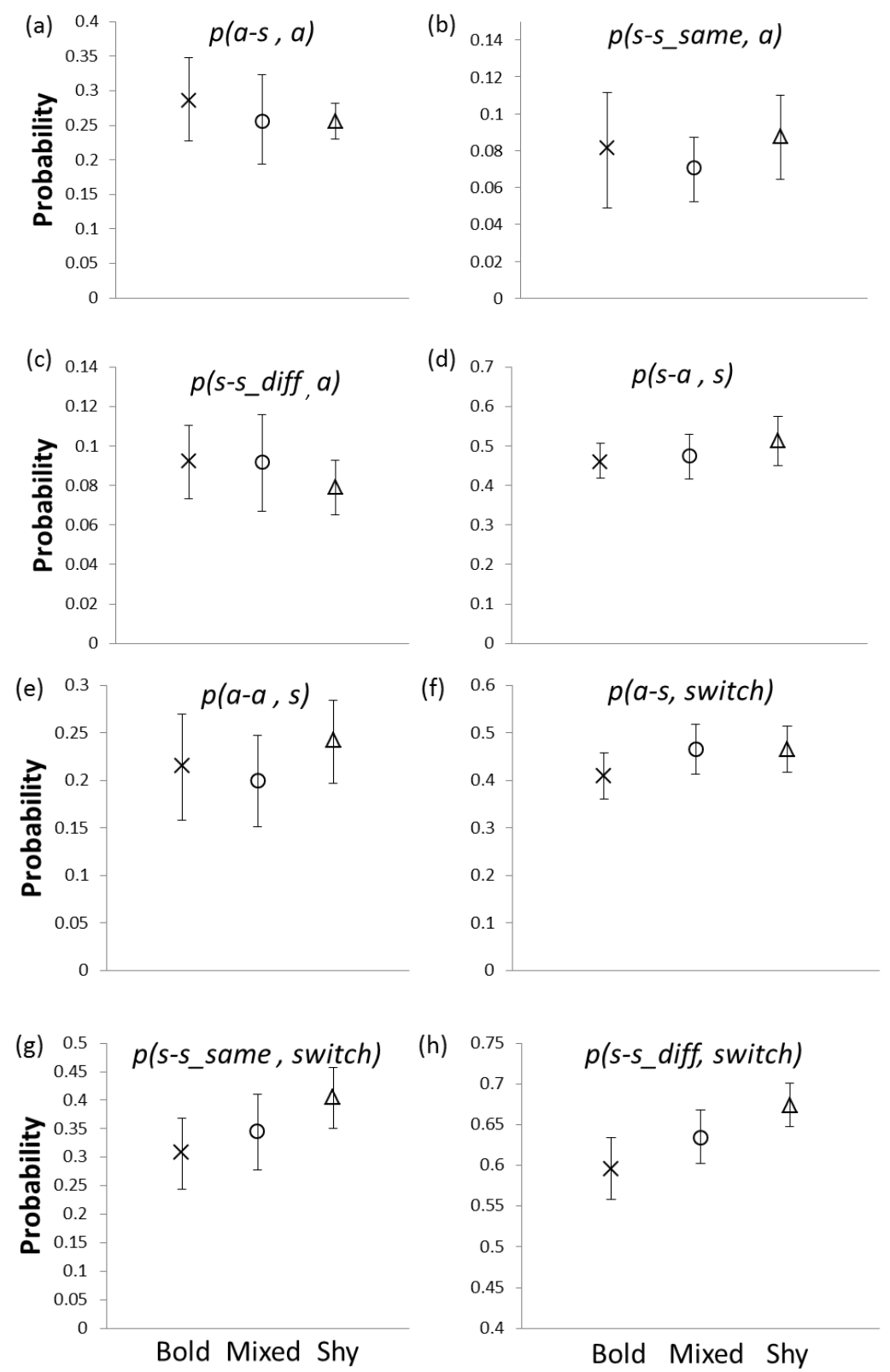

Figure 4.6 Weighted mean transition probabilities and 95\% confidence intervals for the second-order Markov chain models. Bold-dominated groups are denoted by $x$ 's, mixed groups by circles, and shy-dominated groups by triangles; $n=12$ groups per treatment. Shown are the probabilities: (a) $p(a-s, a)$, (b) $p\left(s-s \_s a m e, a\right)$, (c) $p\left(s-s \_d i f f, a\right)$, (d) $p(s-a, s),(\mathrm{e}) p(a-a, s),(\mathrm{f}) p(a-s, s w i t c h),(\mathrm{g}) p\left(s-s \_s a m e, s w i t c h\right)$, and (h) $p\left(s-s \_d i f f\right.$, switch). 
190.69, df $=24, P<0.001$; shy-dominated: $X^{2}=194.29$, df $\left.=24, P<0.001\right)$. As such, network edges within association networks were weighted by this latter measure.

\section{4 (G)LMM Analysis of Individual Activity Levels and Shoaling Tendencies}

The interaction between individual personality and group composition did not influence an individual's activity level and was removed from the model to permit testing of main effects $\left(\mathrm{X}^{2}=0.94, \mathrm{df}=2, P=0.625\right)$. An individual's activity level was not dependent on personality type $\left(\mathrm{X}^{2}=0.58, \mathrm{df}=1, P=0.446\right)$, group composition $\left(\mathrm{X}^{2}=\right.$ $3.28, \mathrm{df}=2, P=0.194)$, or body length $\left(\mathrm{X}^{2}=0.16, \mathrm{df}=1, P=0.689\right)$. The interaction between personality and group composition did not affect the amount of time an individual spent alone and was removed from the model to permit testing of main effects $\left(\mathrm{X}^{2}=1.54 \mathrm{df}=2, P=0.463\right)$. An individual's time spent alone was not impacted by its personality $\left(\mathrm{X}^{2}=1.12\right.$, df $\left.=1, P=0.29\right)$, group composition $\left(\mathrm{X}^{2}=1.02\right.$, $\mathrm{df}=2, P=$ $0.601)$, or body length $\left(\mathrm{X}^{2}=1.04, \mathrm{df}=1, P=0.308\right)$. Finally, regarding an individual's mean group size when shoaling, there was no effect of the interaction between group composition and individual personality $\left(\mathrm{X}^{2}=0.65, \mathrm{df}=2, P=0.723\right)$. However, while neither group composition $\left(\mathrm{X}^{2}=2.83\right.$, $\left.\mathrm{df}=2, P=0.243\right)$ nor body length $\left(\mathrm{X}^{2}=0.06, \mathrm{df}=\right.$ 1, $P=0.808$ ) influenced mean group size when shoaling, an individual's personality type had a significant effect $\left(\mathrm{X}^{2}=5.40, \mathrm{df}=1, P=0.020\right)$. Shy individuals tended to shoal in slightly larger groups than bold individuals (Table 4.1). Parameter estimates, standard errors, and $95 \%$ confidence intervals for each of the above models are provided in Table 4.1.

\subsection{Phenotypic Assortment}

The association networks for bold-dominated groups were significantly 
Table 4.1 (G)LMM parameter estimates, standard errors, and 95\% confidence intervals ${ }^{a}$

\begin{tabular}{|c|c|c|c|}
\hline & Estimate & SE & $95 \% \mathrm{CI}$ \\
\hline \multicolumn{4}{|l|}{ Activity level (Neg. bin. GLMM) } \\
\hline Intercept & 2.75 & 0.12 & $2.51,3.0$ \\
\hline Mixed groups & 0.21 & 0.17 & $-0.13,0.54$ \\
\hline Shy-dominated groups & 0.32 & 0.18 & $-0.03,0.67$ \\
\hline Shy personality & -0.06 & 0.08 & $-0.23,0.10$ \\
\hline Body length $(\mathrm{cm})$ & 0.02 & 0.05 & $-0.08,0.12$ \\
\hline Negative binomial dispersion parameter & 2.36 & 0.22 & \\
\hline \multicolumn{4}{|l|}{ Time spent alone (Neg. bin. GLMM) } \\
\hline Intercept & 2.94 & 0.09 & $2.76,3.12$ \\
\hline Mixed groups & 0.08 & 0.13 & $-0.18,0.33$ \\
\hline Shy-dominated groups & -0.06 & 0.14 & $-0.33,0.22$ \\
\hline Shy personality & -0.10 & 0.09 & $-0.28,0.08$ \\
\hline Body length $(\mathrm{cm})$ & -0.05 & 0.05 & $-0.14,0.05$ \\
\hline Negative binomial dispersion parameter & 1.85 & 0.16 & \\
\hline \multicolumn{4}{|l|}{ Group size when shoaling (LMM) } \\
\hline Intercept & 0.42 & 0.02 & $0.38,0.46$ \\
\hline Mixed groups & 0.004 & 0.03 & $-0.06,0.06$ \\
\hline Shy-dominated groups & 0.05 & 0.03 & $-0.01,0.11$ \\
\hline Shy personality & 0.02 & 0.01 & $0.004,0.04$ \\
\hline Body length $(\mathrm{cm})$ & 0.001 & 0.01 & $-0.01,0.01$ \\
\hline
\end{tabular}

$a$ : Model estimates were obtained from models in which the interaction between group

composition and individual personality had been removed to permit testing of main effects; this interaction did not have a significant effect on any of the dependent variables. Group identity was included as a random effect in all models. Bold-dominated groups and individuals with bold personalities served as the overall baseline. $n=359$ individuals. 
disassorted with regards to personality type (Table 4.2), meaning that individuals tended to associate with group members exhibiting dissimilar personalities. Patterns of assortativity in mixed and shy-dominated groups did not differ from null expectations (Table 4.2).

\subsection{Network-Based Diffusion Analysis (NBDA)}

To be included in the TADA, a group needed to contain at least two individuals who solved the task, resulting in 10 bold-dominated groups, 11 mixed groups, and 12 shy-dominated groups. Out of the set of 256 fitted models, two models received $>99 \%$ of the summed Akaike weights; in terms of their specification, these two models differed only in that the top model included a non-constant baseline solving rate that systematically increased over time, while the second-ranked model included a constant baseline solving rate. In all other respects, the conclusions drawn from each model are identical. As such, only the results of the top-ranked model—which accounted for $68 \%$ of the total Akaike weights_-are presented.

Inclusion of the association networks suggests that the strength of association between two individuals predicted the likelihood of information transmitting between them. The social transmission parameter, $s$, indicates the linear increase in a naïve individual's solving rate per unit of network connection with informed individuals relative to the average asocial solving rate (Hoppitt \& Laland, 2013). The effects of social transmission were weakest in bold-dominated groups ( $s=0.01,95 \%$ CI: 0.007, 0.02), with only $20.9 \%$ of foraging task solves estimated to have resulted from social transmission (Table 4.3; Figure 4.7). Social transmission rates were higher in both shydominated $(s=0.03,95 \%$ CI: $0.02,0.04)$ and mixed groups $(s=0.10,95 \%$ CI: 0.08 , 
Table 4.2 Mean assortativity coefficients $\left(r^{w}\right)$ with regards to personality type

\begin{tabular}{lccc}
\hline Group personality composition & $r^{w}$ & SE & $P$ value $^{a}$ \\
\hline Bold-dominated & -0.15 & 0.08 & 0.015 \\
Mixed & -0.12 & 0.12 & 0.305 \\
Shy-dominated & -0.12 & 0.07 & 0.193 \\
\hline
\end{tabular}

$a: P$ values are 2 -tailed and calculated from comparing observed values to null distributions generated from $10^{4}$ sets of randomized networks.

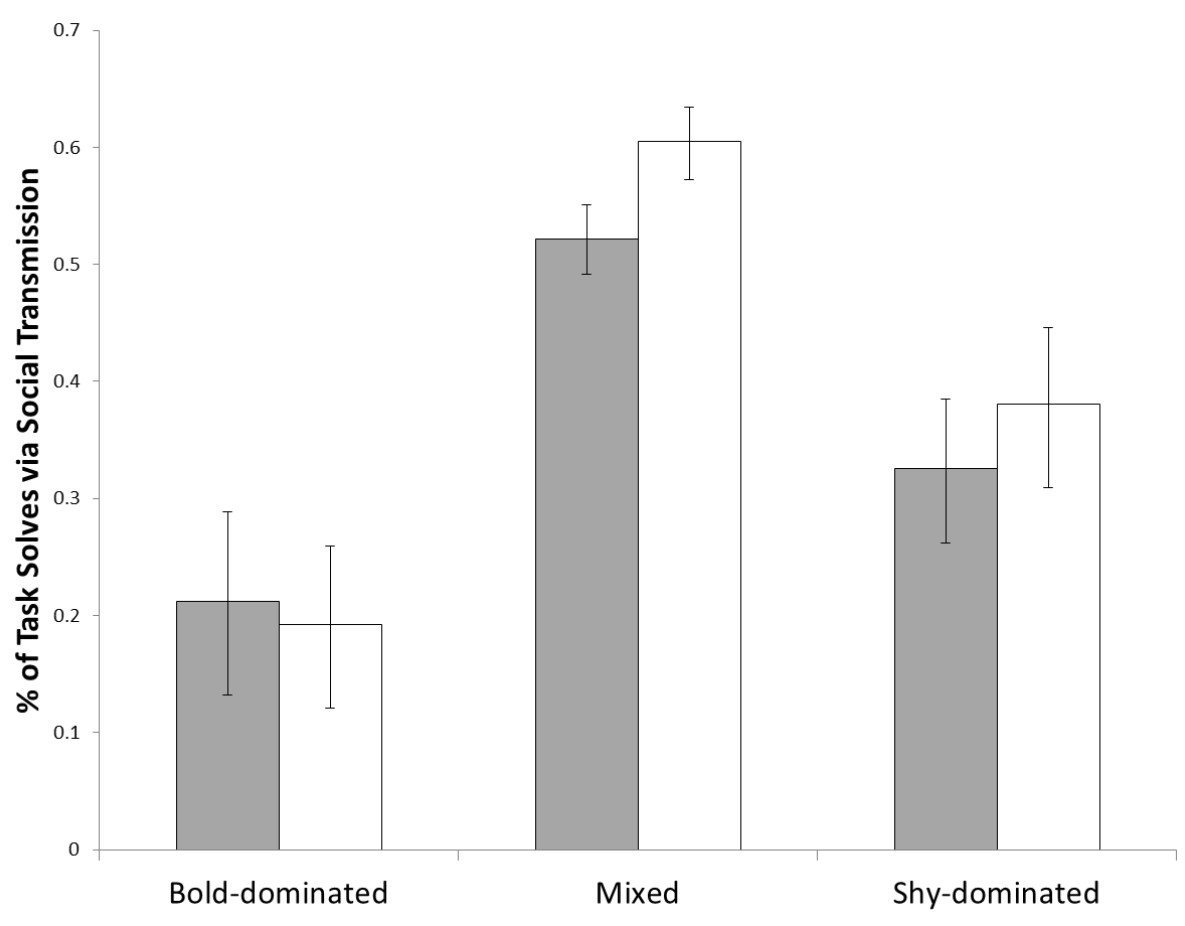

Figure 4.7 The estimated percentage of solving events that resulted from social transmission. Error bars were computed from the $95 \%$ confidence intervals for the social transmission parameters. Grey bars indicate bold individuals, while white bars denote shy individuals. In bold-dominated groups, there were 77 solving events (64 bold individuals; 13 shy individuals). In mixed groups, there were 96 solving events (48 bold individuals; 48 shy individuals). In shy-dominated groups, there were 111 solving events (23 bold individuals; 88 shy individuals). 
0.13), respectively corresponding to an estimated $36.9 \%$ and $56.3 \%$ of solves resulting from social transmission (Table 4.3; Figure 4.7). Within mixed groups, shy individuals were more likely to solve the task via social transmission than were bold individuals, though this pattern was not present in bold- or shy-dominated groups (Figure 4.7). The difference in $s$ was estimated to be 0.08 (95\% CI: 0.06, 0.10) between mixed and shydominated groups, 0.09 (95\% CI: 0.07, 0.12) between mixed and bold-dominated groups, and 0.01 (95\% CI: 0.003, 0.02) between shy- and bold-dominated groups; given that these confidence intervals do not overlap with 0 , this provides strong evidence supporting an effect of group personality composition on social transmission rates (Figure 4.7).

A shape parameter $>1$ indicates that the baseline solving rate increased over time, meaning individuals were more likely to solve the foraging task as the time since its introduction increased (Table 4.3). The top model was multiplicative, meaning that the individual-level variables affected both baseline solving rates and social transmission. While social transmission was strongest in mixed groups, individuals in mixed groups were slower to initially solve the task relative to those in bold- or shy-dominated groups (Table 4.3; Figure 4.8). In contrast, shy-dominated groups had a higher baseline solving rate relative to bold-dominated and mixed groups (Table 4.3, Figure 4.8). All else being equal, bold individuals were faster than shy individuals to solve the novel task (Table 4.3, Figure 4.8), while increasing body length was associated with a decreased solving rate (Table 4.3).

As an additional method to assess the level of support for potential pathways of information flow, I obtained the change $(\Delta)$ in AICc for the top-ranked model when the association networks for a treatment were replaced with alternative network types. 
Table 4.3 TADA parameter estimates, standard errors, and $95 \%$ confidence intervals $^{a}$

\begin{tabular}{|c|c|c|c|}
\hline & Estimate & SE & $95 \% \mathrm{CI}$ \\
\hline \multicolumn{4}{|l|}{ Social transmission strength, $s$} \\
\hline Bold-dominated ( $n=10$ groups) & 0.01 & 0.004 & $0.007,0.02$ \\
\hline Mixed ( $n=11$ groups) & 0.10 & 0.01 & $0.08,0.13$ \\
\hline Shy-dominated ( $n=12$ groups) & 0.03 & 0.01 & $0.02,0.04$ \\
\hline \multicolumn{4}{|l|}{ Individual-level variables $^{b}$} \\
\hline Mixed groups' baseline solving rate & -0.44 & 0.09 & $-0.63,-0.26$ \\
\hline Shy-dominated groups' baseline solving rate & 0.24 & 0.09 & $0.09,0.41$ \\
\hline Bold personality & 0.22 & 0.04 & $0.13,0.30$ \\
\hline Body length (cm) & -0.07 & 0.02 & $-0.11,-0.04$ \\
\hline
\end{tabular}

$a$ : The shape parameter was 1.09 , indicating a baseline solving rate that systematically increased over time. The top model used the multiplicative TADA variant, meaning that the individual-level variables affected both an individual's baseline solving rate and social transmission.

$b$ : Parameter estimates for the individual-level variables provide the additive effect on the $\log$ scale on the baseline solving rate for an increase in one unit for that variable. To obtain an individual-level variable's multiplicative effect on the solving rate, it must be back-transformed. For example, if individual $\mathrm{A}$ is $2 \mathrm{~cm}$ longer than individual B, A will solve the task $\exp (-0.07 \times 2)=0.87$ times slower than B. Likewise, all else being equal, bold individuals solved the task $\exp (0.22)=1.25$ times as fast as shy individuals. The baseline solving rates for bold-dominated groups and for shy individuals were used as the overall baseline-i.e., they equaled 0 . 
Negative values for $\triangle \mathrm{AICc}$ indicate an improved model fit, while positive values greater than 10 indicate that there is essentially no support for that model (Burnham \& Anderson, 2002). Replacement of the association networks with bold-leader networks resulted in a significant improvement in fit for bold-dominated groups $(\triangle \mathrm{AICc}=-26.6)$, but received no support in mixed $(\triangle \mathrm{AICc}=163.4)$ and shy-dominated groups $(\triangle \mathrm{AICc}=17.4)$.

Similarly, inclusion of the shy-social-learner networks in bold-dominated groups improved model fit $(\triangle \mathrm{AICc}=-8.6)$, but received no support in mixed groups $(\triangle \mathrm{AICc}=$ $102.4)$ and only weak support in shy-dominated groups $(\triangle \mathrm{AICc}=2.4)$. Finally, replacement of the association networks with homogeneous networks resulted in significant decreases in model fit for bold-dominated $(\triangle \mathrm{AICc}=11.4)$ and mixed groups $(\triangle \mathrm{AICc}=97.4)$, while model fit was substantially improved for shy-dominated groups $(\triangle \mathrm{AICc}=-9.6)$.

\section{Discussion}

Individual- and group-level variation in personality can shape the nature and outcomes of social interactions (Webster \& Ward, 2011; Farine, Montiglio, et al., 2015), while fine-scale patterns of association can play an important role in mediating a variety of social processes (Hasenjager \& Dugatkin, 2015; Krause et al., 2015). Using recently developed social network approaches, I have demonstrated the impact of group personality composition over elements of fission-fusion dynamics—in particular, how frequently individuals come into contact with different associates—as well as on patterns of assortment within guppy shoals. Furthermore, group- and individual-level variation in boldness influenced the initial acquisition of novel foraging information, how rapidly this information spread between group mates, and the likely transmission pathways. Given 


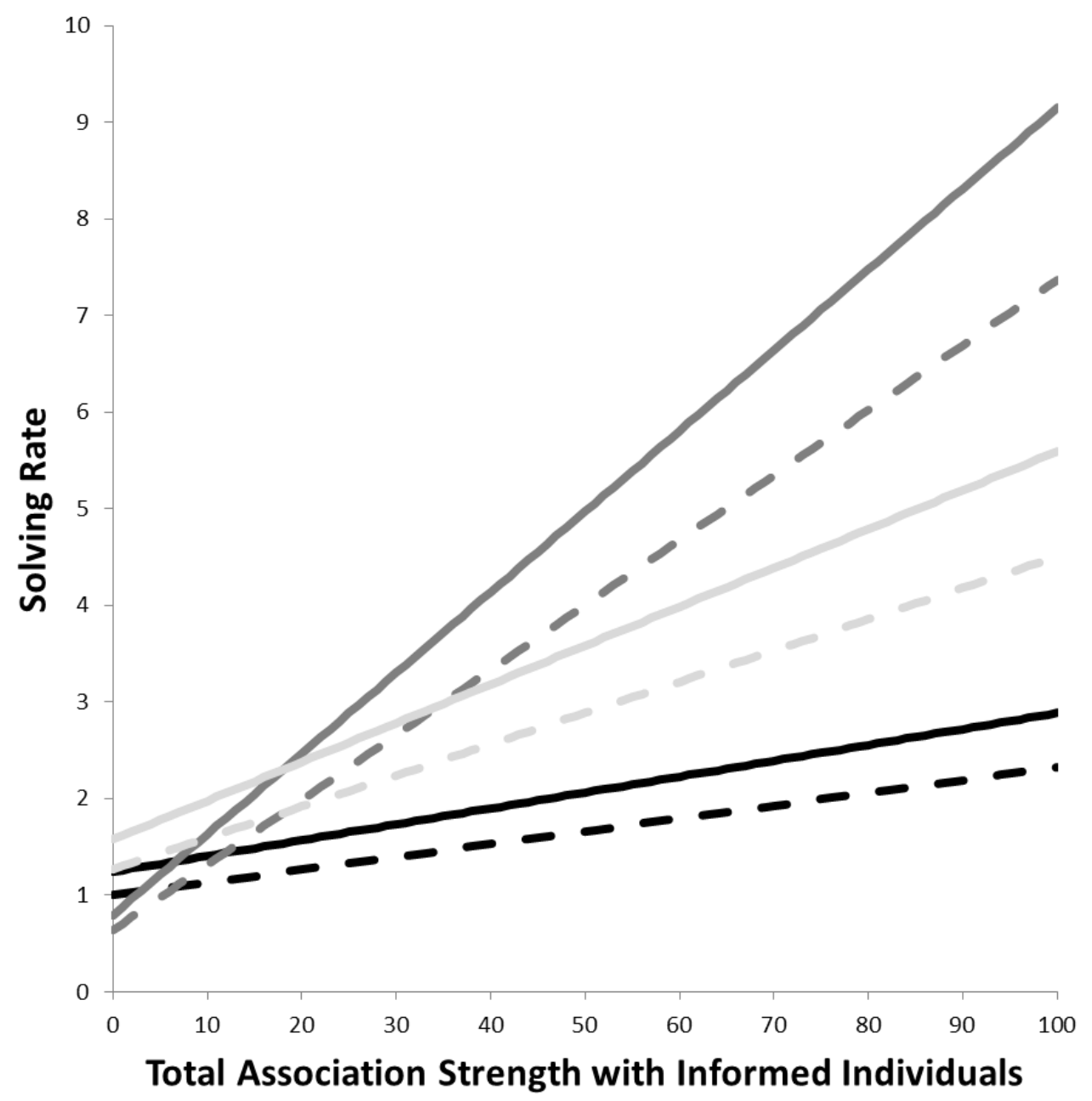

Figure 4.8 The predicted rate at which a naïve individual will solve the foraging task at time $t$ as a function of their total connectedness to informed group members. Both social transmission and baseline solving rates varied based on group personality composition; the predicted solving rate was plotted separately for bold-dominated groups (black lines), mixed groups (dark grey lines), and shy-dominated groups (light grey lines). In addition, the baseline solving rate differed between bold individuals (solid lines) and shy individuals (dotted lines). The estimated solving rates shown here assume an individual of mean body length. 
that access to social information can be important for adaptive decision-making (Danchin et al., 2004; Valone, 2007), the mixture of personality types within an individual's local environment has the potential to greatly impact its survival and reproductive success.

Bolder individuals tend to exhibit decreased social attraction towards group mates, as well as less temporally stable associations (Harcourt et al., 2009; Kurvers et al., 2012; Aplin et al., 2013). As a result, fragmentation can occur within groups composed predominately of bolder individuals (Dyer et al., 2009; Michelena et al., 2009). In contrast to this earlier work, I found no effect of group personality composition on a guppy's probability of initiating or ending bouts of shoaling, nor did group composition or individual personality determine how much time an individual spent alone. However, guppies in bold-dominated groups tended to switch between shoaling partners less frequently than those in shy-dominated groups. This finding may have resulted from bold fish shoaling in slightly smaller groups than shy individuals and thereby having fewer opportunities to switch partners (assuming shifts in group membership occurred at a relatively lower rate). As interaction frequency can determine how rapidly information spreads through a group (Pinter-Wollman et al., 2011), such shoaling dynamics plausibly contributed to the comparatively weak social transmission effect in bold-dominated groups; i.e., uninformed individuals may have been less likely to come into contact with knowledgeable group mates. On the other hand, such an effect could also lower an individual's risk of infection by reducing the likelihood of associating with infected conspecifics (Drewe \& Perkins, 2015). The extent to which the benefits of increased access to information are traded off against a greater risk of infection, and how this trade- 
off might impact an individual's network position, represents an intriguing avenue for future research.

The lack of an effect of group composition on the probability of individuals initiating or terminating bouts of shoaling may pertain to the relationship between the focal personality trait and the social environment. Studies quantifying both boldness and shoaling tendency in the guppy have reported varied results regarding whether these personality traits form a behavioral syndrome or are independent of one another (Croft et al., 2009; Smith \& Blumstein, 2010; Brown \& Irving, 2014). While boldness measures were repeatable in the experimental subjects, this measure of boldness appears to have only weakly reflected shoaling tendencies. Consistent between-individual variation in sociability has been documented in several species (Cote \& Clobert, 2007; Croft et al., 2009; Cote et al., 2012), and within-group variation in this trait can influence group-level outcomes (Brown \& Irving, 2014; Laskowski \& Bell, 2014). Direct manipulation of group composition in terms of sociability could reveal stronger influences over fissionfusion behavior.

Individual variation in behavioral tendencies may also have been masked by the social environment. I assayed individuals' boldness in an asocial context, but betweenindividual variation may have been suppressed when guppies were placed within groups. While some studies have found that individual differences in personality are maintained under social conditions (Brown \& Irving, 2014; Laskowski \& Bell, 2014), this is not always so (Webster, Ward, et al., 2007; McDonald et al., 2016). Whether personality traits are expressed within a group context can depend on aspects of the social environment, as well as the behavioral context (Webster \& Ward, 2011; McDonald et al., 
2016). For example, within three-spined stickleback (Gasterosteus aculeatus) shoals, bolder individuals were the first to emerge from a refuge, but the subsequent decision to travel over open water towards a foraging site was not based on leadership by bold fish, but rather on consensus decision-making (McDonald et al., 2016). In the current study, while individual- and group-level personality variation had only moderate effects over certain elements of shoaling behavior, these factors strongly influenced both the acquisition and social transmission of foraging information.

It is also possible that the behavioral assay used in this study did not measure boldness per se, but instead measured some other personality trait, such as activity level or anxiety. However, the activity level measured for focal individuals in the arena was unrelated to their assigned personality type (bold or shy), suggesting my behavioral assay did not simply measure an individual's movement tendencies. Responses to threatening stimuli-such as the latency to resume normal behavior (as used here) or predator inspection behavior - have often served as measures of boldness (Dugatkin \& Alfieri, 2003; Pike et al., 2008; Croft et al., 2009), though it has recently been suggested that such responses might reflect anxiety in some cases (Carter et al., 2012). For example, chacma baboons (Papio ursinus) that exhibited strong alarm responses to a model snake also spent longer inspecting it, possibly in order to better assess the level of threat it represented (Carter et al, 2012). If anxious individuals are more motivated to acquire information about potential threats, then in the context of the current study, such individuals might be more likely to inspect, and eventually solve, the novel foraging task. At present, anxiety is not well-integrated into animal personality research (Réale et al., 
2007). Further work is needed to examine the interplay between anxiety and other personality traits, and to establish its impacts on social structure and group dynamics. Randomization tests that controlled for the number and duration of contact phases found that individuals in all treatments exhibited non-random nearest neighbor preferences. These preferences were expressed in terms of the number of contact phases shared between two individuals, rather than the mean duration of contact between them. This is consistent with previous work on this species (Wilson et al., 2014; Hasenjager \& Dugatkin, 2017). However, more data may be required to detect whether nearest neighbor preferences are also expressed in terms of contact duration, as contact phases of longer duration occurred less frequently. Additionally, single bouts of shoaling between two individuals were often broken up into several contact phases due to the fact that only the focal individual's nearest neighbor-rather than all nearby group members—was recorded at each time point.

Association networks weighted by the number of shared contact phases between group members tended to be disassortative with regards to boldness, though this was only significant in bold-dominated groups. Positive assortment according to personality type has been observed in wild guppy shoals (Croft et al., 2009) and between male great tits (Parus major) (Aplin et al., 2013), likely driven in part by the strong social attraction exhibited by shy, more reactive individuals in these systems. In contrast, while shy guppies in the experimental groups shoaled in slightly larger groups than bold individuals, they were not any more likely to be engaged in shoaling per se, making positive assortment between shy individuals less likely. 
Disassortative patterns in bold-dominated groups could have resulted from boldness correlating with leadership tendencies (Harcourt et al., 2009; Kurvers et al., 2012), thus promoting association between bold leaders and shy followers. Consistent with this idea, inclusion of both the bold-leader and shy-social-learner networks was favored in bold-dominated groups, potentially indicating that knowledgeable bold individuals were more likely to lead naïve group members to the food source, while shy individuals were more likely to follow and thus acquire the task solution. Conversely, there was little support for the bold-leader networks or disassortative mixing in shydominated and mixed groups. This may suggest that group personality composition affects the adoption of social roles during collective behaviors and/or the extent to which key individuals—-such as informed group mates — can drive collective outcomes (Modlmeier, Keiser, et al., 2014; Nakayama et al., 2016).

Information transfer within fish shoals can occur through relatively simple social learning mechanisms, such as local enhancement (Thorpe, 1956; Hoppitt \& Laland, 2013). For example, knowledgeable individuals can transmit information to naïve group members by acting as leaders during collective movements (Swaney et al., 2001; Webster et al., 2013; Ioannou et al., 2015) or by otherwise directing attention towards relevant environmental stimuli—e.g., postural changes or foraging pecks can indicate the presence of food (Lachlan et al., 1998). In addition, olfactory cues associated with foraging may provide a tip-off to uninformed individuals regarding the presence of prey items (Morrell et al., 2007; Johannesen et al., 2012). Within the experimental groups, I found strong support for social transmission of the foraging task solution. Furthermore, consistent with the hypothesis of directed social learning (Coussi-Korbel \& Fragaszy, 1995), the 
likelihood of information transferring between two individuals was correlated with how frequently they tended to associate together - that is, the spread of the task solution followed the pattern of connections within the association networks.

Bold individuals acquired the task solution more rapidly than shy fish, as evidenced by a higher baseline solving rate. Boldness signifies a greater willingness to accept risk in return for potential reward (Réale et al., 2007), suggesting that bold individuals may have been especially prone to approach the device and/or risk visual disruption with group mates by entering it. This finding parallels earlier work indicating that bold individuals are more likely to engage in personal sampling of the environment and tend to act as information producers within a social group (Dyer et al., 2009; Kurvers et al., 2012). These behavioral tendencies may be especially important when groups encounter novel situations, such as when dispersing into a new environment (Cote et al., 2010; Chapple et al., 2012; Wolf \& Weissing, 2012), or when assessing the level of risk posed by a potential predator (Dugatkin, 1991; Croft et al., 2006). Individuals were also more likely to solve the task as time progressed after its introduction, while smaller fish exhibited a higher solving rate. This latter finding is consistent with prior studies reporting a link between body size and risk-taking in fish (Krause et al., 1998; Dyer et al., 2009; though see Harris et al., 2010), potentially as a result of increased metabolic demands within smaller individuals (Krause et al., 1998; Brown \& Braithwaite, 2004). While bold individuals acquired the task solution more rapidly, shy individuals may have been more likely to use social information to solve the task. Within mixed groups, more shy individuals were estimated to have solved the task as a result of social transmission than bold fish, while there was some support in the bold-dominated groups 
for the shy-social-learner networks, suggesting that social information may have transmitted to shy individuals at a higher rate. Shyness has been associated with increased acquisition and/or use of social information after controlling for grouping tendencies (Kurvers et al., 2010), potentially as a result of greater social responsiveness or attentiveness towards group mates (van Oers et al., 2005; Jolles et al., 2015). However, my results indicate that whether shy individuals rely on social information to a greater extent than bold individuals may depend on elements of their social environment - e.g., some social contexts may offer greater opportunities to observe or interact with knowledgeable individuals than others. The extent to which individuals might select their social environment in order to maximize their access to information or other social resources is a fascinating topic for future work (Oh \& Badyaev, 2010; Saltz et al., 2016).

Fish in shy-dominated groups were more likely to solve the task than those in bold-dominated or mixed groups, all else being equal. Given the tendency of shy individuals to travel in somewhat larger groups, it may be that fish in shy-dominated groups were more likely to approach the device as part of a larger, more cohesive shoal. While the estimated difference in mean group size between bold and shy fish was modest, the introduction of a novel (potentially threatening) stimulus might have resulted in a more pronounced difference between personality types in this regard (Dyer et al., 2009; McDonald et al., 2016). Increases in group size can promote exploration (Brown \& Irving, 2014) and investigation of potential threats (Dugatkin, 1991; Croft et al., 2006) by diluting the risks to each individual and by enhancing anti-predator defenses (Krause \& Ruxton, 2002). As a result, this social facilitation effect could promote exploration and eventual solving of the foraging task (Zajonc, 1965; Hoppitt \& Laland, 2013). Thus, 
while bold individuals were faster to acquire novel information individually, grouping may provide a means for shy individuals to acquire information at a similar rate, though potentially at the cost of increased competition over resources (Metcalfe \& Thompson, 1995; Krause \& Ruxton, 2002).

In addition, if individuals prefer to remain in close proximity to the device once they have acquired the task solution (or are more likely to return to it), this could result in social transmission of the solution via local enhancement (Thorpe, 1956; Hoppitt \& Laland, 2013). Such an effect would be consistent with the multiplicative TADA model used here, in which the higher baseline solving rate in shy-dominated groups also resulted in higher rates of social transmission. The support for the inclusion of the homogeneous networks within shy-dominated groups provides further evidence consistent with the above interpretation; individual variation in network strength is less likely to matter for social transmission if individuals interact with the device as part of a larger group (Webster et al., 2013). Rather than implying a lack of social structure, homogeneous networks under such circumstances may reflect the relative ease of maintaining visual contact with each group member, regardless of nearest neighbor preferences.

Social transmission rates were strongly dependent on group personality composition. Within bold-dominated groups, increasing numbers of informed individuals had little influence over the probability that naïve group members would solve the task, resulting in most solving events occurring primarily through individual learning. Conversely, connectedness to knowledgeable associates strongly accelerated the rate at which naïve fish became informed in mixed groups and, to a lesser extent, shy-dominated ones. Thus, while bold individuals appear to act as information producers, shy individuals 
may facilitate the rapid transmission of information through a group — such as by occupying larger group sizes than bold individuals on average or through greater attentiveness to social cues (Kurvers et al., 2010; Jolles et al., 2015). However, social transmission rates were substantially higher in mixed groups, pointing to synergistic influences between personality types.

Within-group diversity in personality can enhance group-level outcomes as a result of personality types shaping group dynamics in complementary ways (Dyer et al., 2009; Pruitt \& Riechert, 2011). For example, boldness often maps onto leader-follower tendencies (Harcourt et al., 2009; Aplin et al., 2014), such that social transmission may be enhanced by pairing informed bold leaders with individuals more likely to acquire social information - that is, shy followers. However, there was little evidence for this relationship within mixed groups, given the lack of support for the bold-leader networks or for disassortment by personality type. Rather than boldness correlating with leaderfollower relationships per se, high social transmission rates may have resulted from mixed groups containing both effective information producers—i.e., bold fish—along with shy individuals that may have been more likely to acquire social information and/or contributed to social conditions that facilitated transmission. Regardless of the mechanism(s) at work, these results are consistent with the idea that phenotypic diversity can facilitate flexible group-level responses to environmental challenges (Burns \& Dyer, 2008; Michelena et al., 2010; Aplin et al., 2014).

Animals use social information in a diverse array of contexts, including the detection of foraging sites (Swaney et al., 2001; Aplin et al., 2012), mate choice (Dugatkin \& Godin, 1993), and the identification of potential threats (Ferrari et al., 2007). 
As such, variation in access to social information has the potential to greatly impact fitness outcomes. Together, these results indicate that group personality composition can have strong effects on social network structure, the initial acquisition of information, and its subsequent diffusion through a group. How individuals respond to the effects of group composition — e.g., modifying their phenotype and/or their social environment—and the evolutionary consequences of such responses remains an exciting area for future research (Farine, Montiglio, et al., 2015). While I focused on boldness, personality is defined by one's position along several behavioral axes (Réale et al., 2007), as well as by correlations across suites of traits-i.e., behavioral syndromes (Sih et al., 2004). To advance our understanding of how social structure and collective outcomes are shaped by personality variation, it will be necessary to examine the simultaneous influence of multiple personality traits across an array of social environments and functional contexts. Thus, integrating animal personalities into the field of collective behavior promises to remain a challenging and rewarding endeavor for some time. 


\section{CHAPTER V}

\section{FEAR OF PREDATION SHAPES SOCIAL NETWORK STRUCTURE AND THE ACQUISITION OF FORAGING INFORMATION}

\section{Introduction}

Predation shapes the ecology of prey species through both lethal and non-lethal effects (Lima \& Dill, 1990; Lima, 1998). Beyond the direct impact of mortality, fear of predation can elicit behavioral responses strong enough to affect the demography and life histories of entire prey populations (Ripple \& Beschta, 2004; Magurran, 2005; Zanette et al., 2011). As the threat of predation is variable across space and time, many prey species express plastic anti-predator defenses, which allow them to respond appropriately to potential threats while minimizing opportunity costs-e.g., reduced time engaged in foraging or courtship behavior (Lima \& Dill, 1990; Brown et al., 2013). Grouping is one such response to increased threat levels, and acts to reduce predation risk through dilution effects and interference with a predator's ability to target individual group members (Landeau \& Terborgh, 1986; Krause \& Ruxton, 2002; Ioannou et al., 2012). Prey species also frequently exhibit phenotypic assortment according to characteristics such as species, body size, and sex (Krause \& Ruxton, 2002) in order to counteract preferential targeting of phenotypically dissimilar individuals by predators (Landeau \& Terborgh, 1986; Rodgers et al., 2015). 
While numerous studies have examined how grouping tendencies vary with predation risk, less is known regarding the potential effects of ambient threat levels on fine-scale patterns of association within groups or populations. Such patterns can be quantified as a social network, in which nodes, representing individuals, are connected by edges denoting some form of social relationship (Pinter-Wollman et al., 2014; Hasenjager \& Dugatkin, 2015). Those studies that have examined the impacts of predation risk on network structure have found that heightened perception of risk results in more densely interconnected networks (Sundaresan et al., 2007; Kelley et al., 2011) and can drive the formation of stable social bonds between group members (Kelley et al., 2011; Heathcote et al., 2017). Such structural shifts in response to the threat of predation can have important implications for a number of social processes. For example, stable social ties can facilitate the maintenance of cooperative relationships (Croft et al., 2006; Micheletta et al., 2012), while high network density is associated with the rapid diffusion of information through a group (Voelkl \& Noë, 2010; Pinter-Wollman et al., 2011). In species exhibiting fission-fusion behavior, the social dynamics from which network structures emerge are also expected to vary according to ambient levels of risk, given their predicted effects on individual exposure to predation (Krause et al., 2016).

In addition to its effects on grouping patterns and social structure, predation risk can also influence the information-gathering strategies employed by prey individuals. Acquiring information can be hazardous in high predation environments, where the cost of a mistake can mean death. Heightened neophobic responses towards novel habitats and stimuli in high-risk environments assist naïve individuals in recognizing and responding appropriately to potential threats-i.e., the dangerous niche hypothesis (Greenberg, 2003; 
Brown et al., 2013) — but at the potential cost of being slower to exploit beneficial opportunities (Elvidge et al., 2016). Even when individuals are not confronting novel stimuli or situations, personal sampling of the environment can be risky in high predation environments, as it may increase the likelihood of encountering a predator or necessitate reductions in vigilance (Lima \& Dill, 1990; Webster \& Laland, 2008).

In contexts where the collection (or use) of personal information is costly, theory and empirical results suggest that individuals should rely on social information to a greater extent (Boyd \& Richerson, 1985; Mineka \& Cook, 1988; Laland, 2004). For instance, European minnows (Phoxinus phoxinus) with personal information about foraging patch quality switched their patch preference after observing demonstrators at a previously unprofitable patch, but only under high risk conditions (Webster \& Laland, 2008). As such, while the asocial acquisition of novel information may be inhibited in high risk environments, once it has been acquired, greater reliance on social information could result in its rapid diffusion via social transmission. However, little is known regarding how these processes interact to affect the overall rate of diffusion and the likely pathways of information flow within social groups.

Here, I manipulated the ambient level of predation risk experienced by shoals of Trinidadian guppies (Poecilia reticulata) under captive conditions in order to examine its impacts on: (i) their social network structure, (ii) the fission-fusion dynamics which generate that structure, and (iii) the initial acquisition and subsequent transmission of novel foraging information. The guppy possesses several characteristics that make it an ideal system for the present study. Social network approaches have been extensively applied to probe the causes and consequences of variation in guppy social biology (Croft 
et al., 2004; Morrell et al., 2008; Croft et al., 2009; Wilson et al., 2015), and the guppy has frequently been used to examine questions related to the acquisition and use of social information (Dugatkin \& Godin, 1993; Swaney et al., 2001; Hasenjager \& Dugatkin, 2017). In addition, the Trinidadian guppy has long served as a model organism for understanding the evolutionary consequences of predation risk (reviewed in Magurran, 2005).

Manipulation of background levels of predation risk was achieved by repeatedly exposing groups of guppies to either distilled water or conspecific alarm cues. In many aquatic species, including the guppy, chemical alarm cues are released following epidermal damage — such as during a predation event — thereby serving as reliable indicators of risk (Chivers \& Smith, 1998). Repeated exposure to alarm cues over several days is sufficient to elevate individuals' perception of background threat levels (Brown et al., 2009; Brown et al., 2013; Chivers et al., 2014). For example, guppies repeatedly exposed to conspecific alarm cues exhibited strong anti-predator responses upon detection of novel odors, while control groups that had been repeatedly exposed to water exhibited no such responses (Brown et al., 2015). Comparative work on wild prey populations living under different levels of predation risk has also shown that behavioral responses elicited in captivity are consistent with those observed in the wild (Brown et al., 2013; Elvidge et al., 2016).

I tested the following predictions. First, high background levels of predation risk would result in prioritization of group cohesion, resulting in individuals being less likely to cease shoaling with conspecifics and spending shorter periods of time alone. Second, groups exposed to high-risk conditions would exhibit stronger phenotypic assortment 
than low-risk groups. Third, exposure to high background predation risk would impede the initial acquisition of the solution to a novel foraging task. Finally, social transmission of the task solution between group mates would occur at a higher rate in high-risk groups relative to low-risk ones.

\section{Methods}

\subsection{Study Subjects and Rearing Conditions}

This study was conducted from July to September 2016 using lab-reared descendants of guppies collected in 2003 from the Quaré River, Trinidad. Juvenile guppies ( $\leq 7 \mathrm{~mm}$ in length) were collected en masse from four 208-L stock tanks-each of which contained several hundred fish - and were randomly assigned to one of 32 rearing tanks (37.9-L) in which they were raised to maturity. Rearing tanks contained 3-7 adult females and no more than 15 juveniles at any time. Males were removed to the stock tanks as they matured. Any females that were not raised within the rearing tanks were not used to construct the experimental groups. Rearing tanks were periodically restocked with juveniles drawn from the stock tanks as described above. Rearing tanks were visually and chemically isolated from one another, filtered, and maintained at 26-27 ${ }^{\circ} \mathrm{C}$ on a 12-h:12-h light-dark cycle. Each contained a layer of gravel $2 \mathrm{~cm}$ deep and artificial plants for shelter. Fish were fed Cobalt Aquatics® Tropical Flake Food daily and supplemented every other day with live Artemia nauplii.

\subsection{Alarm Cue Preparation}

Alarm cue was generated from non-gravid female guppies drawn from four 208-L stock tanks. Females were euthanized via decapitation followed immediately by destruction of brain matter. The tail was removed before placing the body in $50 \mathrm{~mL}$ of 
distilled water. Tissue samples were homogenized, passed through filter floss, and diluted with distilled water to $\sim 0.1 \mathrm{~cm}^{2}$ tissue $\mathrm{mL}^{-1}$. This concentration reliably elicits antipredator responses in guppies (Brown et al., 2013; Brown et al., 2015) and pilot work indicates that this remains true of the laboratory population used here (see below). Alarm cues were frozen at $-80{ }^{\circ} \mathrm{C}$ in $12 \mathrm{~mL}$ aliquots until needed. New alarm cue stock was prepared for each experimental block; for the main study, 126 donors were sacrificed in all, while an additional 6 donors were sacrificed for the pilot work.

\subsection{Pilot Study}

To confirm that our laboratory guppy population responded in species-typical fashion to conspecific alarm cues, I conducted a pilot study prior to the main experiment. Non-gravid adult female guppies were collected from the stock tanks and used to construct groups of three individuals size-matched to within $2 \mathrm{~mm}$ standard length. Groups were then placed within 37.9-L aquaria filled with $27 \mathrm{~L}$ of treated tap water; the sides and back wall of these tanks were covered with opaque plastic. To facilitate quantification of activity level and area use, horizontal lines were drawn on the front wall of the tank to divide it into three equal sections. An airstone was secured along the back wall along with $1.5 \mathrm{~m}$ of airline tubing to allow for injection of alarm cues or water into the tank. Tanks contained $2 \mathrm{~cm}$ gravel substrate and were maintained at $26-27^{\circ} \mathrm{C}$ on a 12-h:12-h light:dark cycle. Groups were fed flake food ad libitum and allowed to acclimate overnight. The following morning, groups were fed once more to ensure satiation; trials were initiated one hour after feeding. Trials were recorded using a Canon Vixia HG21 digital camcorder set up $2 \mathrm{~m}$ in front of the tanks. 
Trials were divided into a five-minute pre-injection and five-minute post-injection period. Prior to a trial, $60 \mathrm{~mL}$ of water was drawn from the tank through the airline tubing and discarded; an additional $60 \mathrm{~mL}$ of water was then drawn and retained in order to flush the stimulus into the tank (see below). During the pre-injection period, I quantified three behavioral variables: activity, area use, and shoaling behavior. To quantify activity, a focal individual was randomly selected and I counted each time that individual moved into a new section of the tank (upper, middle, or lower). Area use was recorded every 15 seconds. Individuals received a 1 if they occupied the lowest area and a 3 if they were in the uppermost region. Area use scores therefore ranged from 3 (all individuals near the substrate) to 9 (all individuals located near the water's surface). Shoaling behavior was recorded every 15 seconds and ranged from 1 (no individuals were within one body length of one another) to 3 (all individuals were within one body length). Following the pre-injection period, groups were exposed to either $10 \mathrm{~mL}$ of conspecific alarm cue or 10 $\mathrm{mL}$ of distilled water; the stimulus was slowly flushed into the tank through the airline tubing using the retained $60 \mathrm{~mL}$ of tank water drawn prior to the trial. Immediately following stimulus injection, behavior was recorded for an additional five minutes as described above. Sixteen groups were tested in total ( $n=8$ per treatment).

\subsection{Testing Procedure}

Due to logistical constraints, only eight groups could be tested at a time. As such, this study was completed in four blocks, with each block representing a cohort of eight groups. Within each block, half of the groups were exposed to high-risk conditions and the other half to low-risk conditions. A new batch of alarm cue substance was prepared for each block. In total, 32 groups were tested ( $n=16$ groups per treatment). 
Groups were constructed from eight adult non-gravid female guppies, raised to maturity in the rearing tanks and each drawn from a separate tank. Only females were used as they are more likely to form stable social relationships (Griffiths \& Magurran, 1998; Croft et al., 2006) and are more food-motivated than males (Reader \& Laland, 2000; Magurran, 2005). Experimental groups were size-matched to $\leq 2 \mathrm{~mm}$ standard length. The mean $( \pm$ SD) length of individuals was $1.94 \pm 0.20 \mathrm{~cm}$. To permit individual identification, guppies were anesthetized with MS-222 buffered with sodium bicarbonate and injected with two colored elastomer tags (Northwest Marine Technologies, Inc.; Shaw Island, WA, U.S.A.). This method has been used extensively with guppies (Croft et al., 2006; Morrell et al., 2008; Hasenjager \& Dugatkin, 2017) and does not appear to influence their shoaling decisions (Croft et al., 2004). Groups were allowed to recover for at least one hour following marking in treated, aerated tap water. No mortalities occurred as a result of this procedure. Following the recovery period, groups were then placed within familiarization tanks and randomly assigned to a treatment.

Familiarization tanks were 37.9-L aquaria filled with $27 \mathrm{~L}$ of water; opaque plastic covered the walls of each tank. Each contained an airstone and $1.5 \mathrm{~m}$ of airline tubing that terminated directly above the airstone. Familiarization tanks were otherwise maintained identically to the rearing tanks. Groups were kept within these tanks for 12 days; this period is sufficient for familiarity to develop between female guppies under captive conditions (Griffiths \& Magurran, 1997). On the final four days of familiarization, groups were exposed three times per day between 8:00 and 18:00 to either $10 \mathrm{~mL}$ of conspecific alarm cues (high-risk) or $10 \mathrm{~mL}$ of distilled water (low-risk). To expose groups to these stimuli, I first drew and discarded $60 \mathrm{~mL}$ of water from the 
tank via the airline tubing before retaining an additional $60 \mathrm{~mL}$ of water. I then injected $10 \mathrm{~mL}$ of alarm cue (or water) into the airline tubing before slowly flushing it into the tank using the $60 \mathrm{~mL}$ of retained water. After 30 minutes of exposure, tanks were given a $50 \%$ water change regardless of treatment. At least 1.5 hours elapsed between subsequent exposures and at least 1 hour separated daily feeding from exposure periods. Similar schedules of alarm cue presentation have been reliably shown to elevate perceived predation risk in several taxa, including guppies (Brown et al., 2013; Chivers et al., 2014; Brown et al., 2015).

Immediately following the final exposure on the last familiarization day, groups were removed from their tank and placed within a test arena. Arenas were black rectangular tanks $(84$ x $51 \mathrm{~cm})$ containing black gravel substrate and water $7 \mathrm{~cm}$ deep (Figure 5.1). Heaters recessed within the substrate maintained the water temperature at $26-27{ }^{\circ} \mathrm{C}$. Fourteen black plastic partitions $10 \mathrm{~cm}^{2}$ in area were arranged perpendicular to one another to form a structurally complex space. Overhead illumination was provided by four $23 \mathrm{~W}$ compact fluorescent lights. Trials were recorded using a Canon Vixia HG21 digital camcorder suspended $1.2 \mathrm{~m}$ above the arena. Black cloth was hung around the arena to minimize disturbance from external visual cues in the room. Groups were allowed to acclimate within the arena overnight.

Testing began at 12:00 on the following day. Groups were filmed for 120 minutes to collect shoaling association data following the methods described in Wilson et al. (2014). Focal individuals were randomly selected and observed continuously for four minutes. Every 10 seconds, I recorded whether that individual was alone-i.e., no group mates were within four body lengths - or whether it was shoaling. If the latter, the 


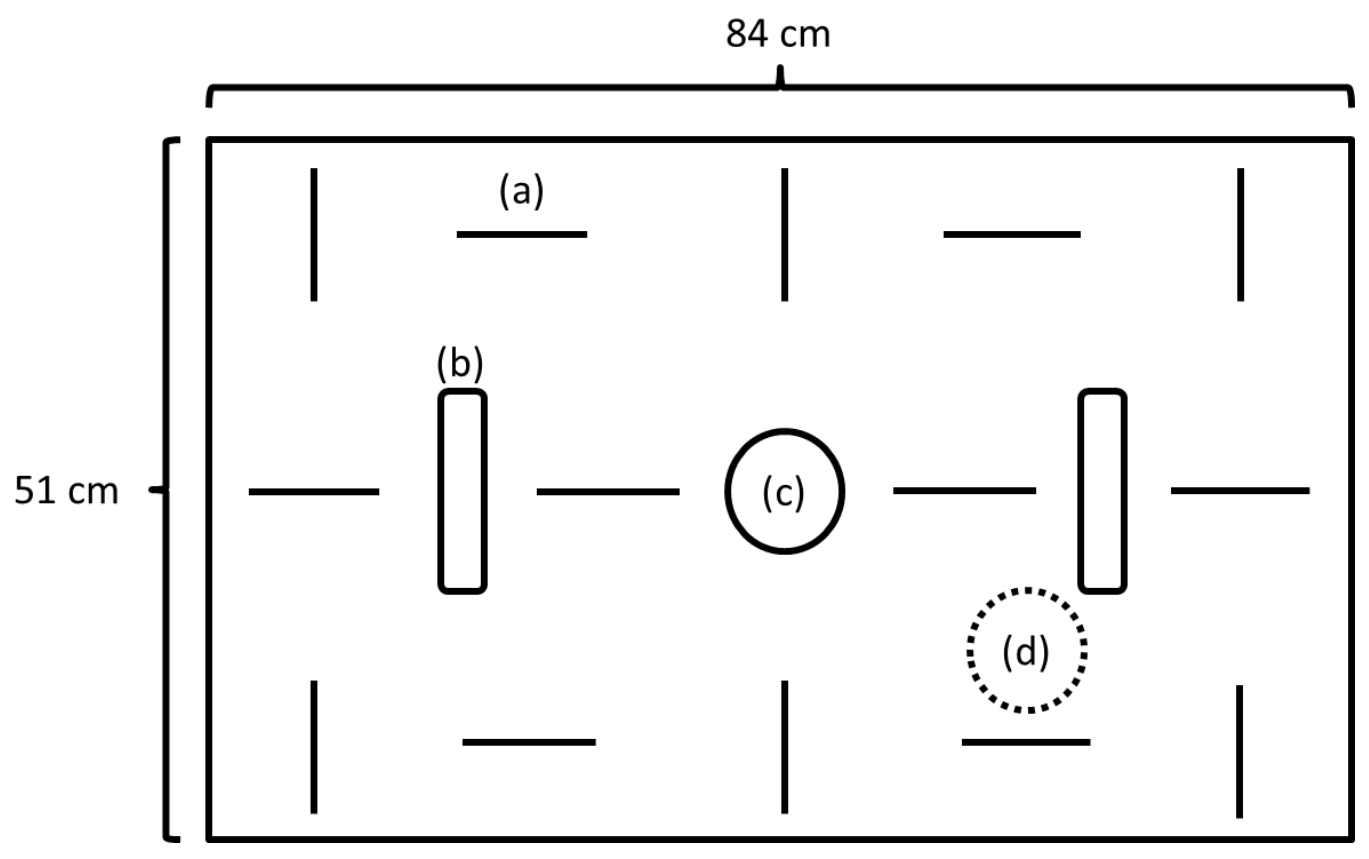

Figure 5.1 Test arena setup. The arena measured 84 x $51 \mathrm{~cm}$. It contained black gravel substrate and treated tap water $7 \mathrm{~cm}$ deep. Fourteen partitions $10 \mathrm{~cm}^{2}$ in area (a) were used to create a structurally complex space, while two heaters (b) maintained water temperature at $26-27^{\circ} \mathrm{C}$. After recording groups for 120 minutes to collect shoaling behavior data, a novel foraging device (c) was introduced into the arena. For those individuals that successfully used the device, their latency to first enter it was recorded. Individual latencies to enter an arbitrary space within the arena of equal diameter to the foraging device (d) were also recorded. 
identity of its nearest neighbor was recorded as measured from the center of their heads. I recorded an individual's activity level as the number of observations in which it had moved $\geq 10 \mathrm{~cm}$ since its prior observation, while its shoaling tendency was recorded as the number of observations in which it was shoaling. After four minutes, a new focal fish was randomly selected until all individuals had been observed in this way. After 12 minutes, a new round of sampling was initiated; this process was carried out three times total, providing 72 observations per individual. Where possible, I avoided selecting focal fish who had been the nearest neighbor of the prior focal individual within the previous 60 seconds.

After 120 minutes, a novel foraging device was gently introduced into the arena (Figure 5.1c). The device was a white PVC tube $8.6 \mathrm{~cm}$ tall and $8.9 \mathrm{~cm}$ in diameter with a grey plastic base and a hole $(2 \mathrm{~cm}$ diameter) drilled into the lower half to permit entry. The cylinder was stocked with 32 freeze-dried bloodworms that floated on the water's surface. These food items were not visible to individuals from outside the device. Groups were filmed for an additional 20 minutes; for those individuals that successfully used the device, I recorded their latency to first enter it. I also recorded each individual's latency to first enter an arbitrary space within the arena with an equivalent diameter to the foraging device (Figure 5.1d). Following testing, groups were returned to the stock tanks and were not used further in this study. Four individuals died during familiarization (two per treatment); thus, four groups of seven fish were included in the analyses.

All experimental procedures, as well as animal care and maintenance protocols, were approved prior to the study's commencement by the University of Louisville's Institutional Animal Care and Use Committee (IACUC \#16547). 


\subsection{Statistical Analyses}

\subsubsection{Pilot Work}

Pre-injection behavior was analyzed with a MANOVA, with shoaling, activity, and area use scores included as response variables and the stimulus to be injected (conspecific alarm cue vs. water) included as an explanatory variable. Activity scores were transformed $[\log ($ Activity*(-1)+303)] to achieve multivariate normality. Subsequently, response variables met assumptions of multivariate normality and homogeneity of variance; there was no indication of multivariate outliers. Baseline behavior did not differ between treatments (see Results), meaning behavioral differences between the pre- and post-injection periods could be examined directly. I calculated these differences for each behavioral measure and used these values as response variables in a second MANOVA. Assumptions of multivariate normality, homogeneity of variance, and a lack of multivariate outliers were met.

\subsubsection{Markov Chain Fission-Fusion Models}

To characterize the shoaling dynamics acting within each treatment, I adopted the approach originally developed by Wilson et al. (2014). A focal individual's shoaling behavior is described by a sequence of behavioral states, where swimming alone is denoted by $a$ and shoaling by $s$. If $a$ and $s$ are regarded as states of a first-order Markov chain, then the probability of transitioning between states is determined solely by an individual's current state at time $t$. The first-order model can be characterized by the probabilities of an individual switching from being alone to shoaling $[p(a, s)]$ and of ceasing to shoal $[p(s, a)]$ (Figure 5.2a). As some behavioral states were not observed with sufficient frequency to permit accurate estimation of individual-specific transition 

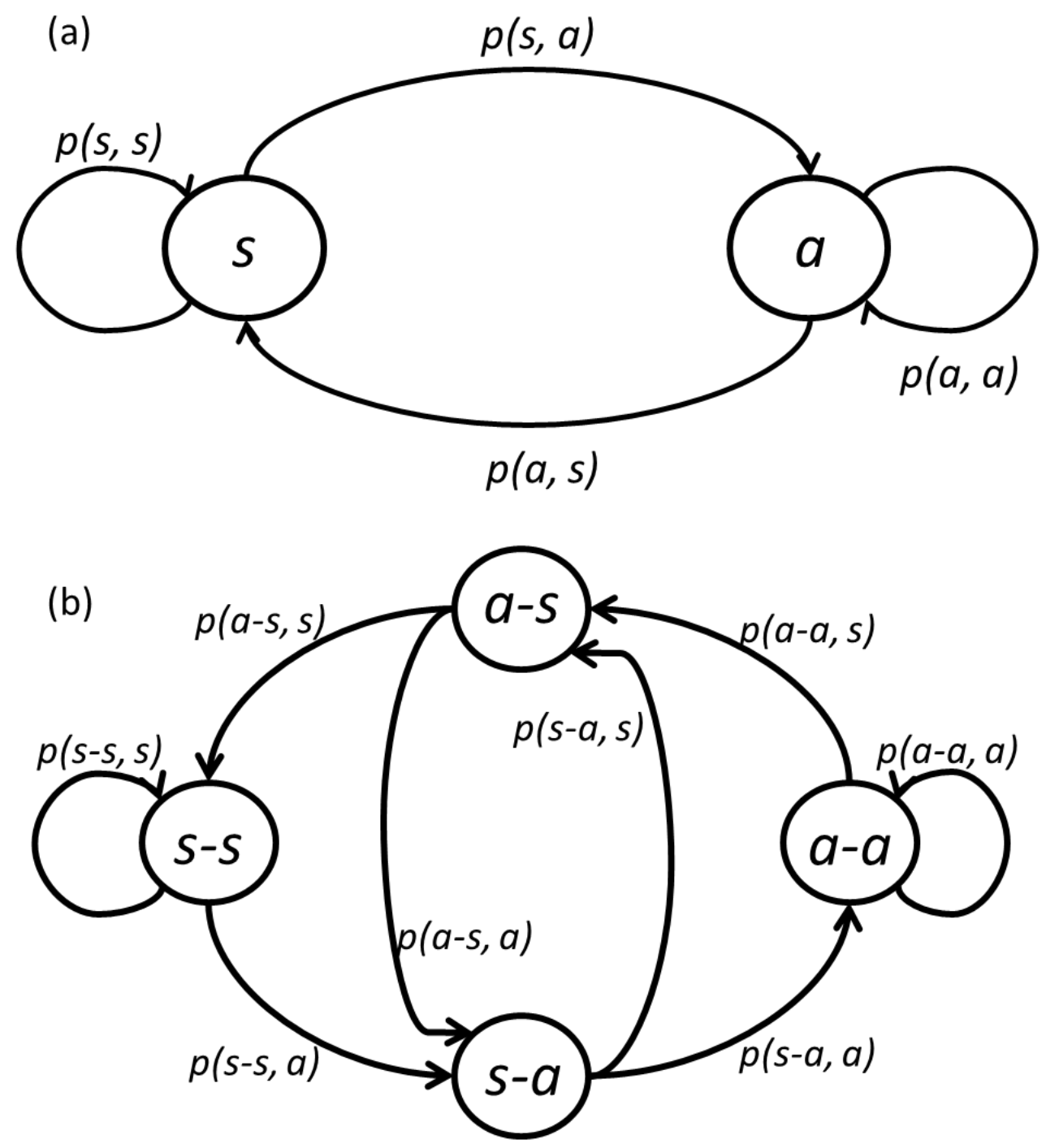
Figure 5.2 Markov chain models of shoaling behavior. (a) In a first-order model, an individual can either be alone $(a)$ or shoaling $(s)$ at time $t$. An individual will move from a shoaling state at time $t$ to an asocial state at time $t+1$ with probability $p(s, a)$ and move from an asocial state to a shoaling state with probability $p(a, s)$. (b) In a second-order model, an individual's state at time $t+1$ is determined jointly by its state at times $t-1$ and $t$. These joint states are depicted in the circles. The model is characterized by four transition probabilities: (i) $p(a-a, s)$, the probability that an individual that has been alone for two consecutive time points will begin to shoal; (ii) $p(s-s, a)$, the probability that an individual that has been shoaling for two consecutive time points will cease to shoal; (iii) $p(a-s, a)$, the probability that an individual that was alone and is currently shoaling will stop shoaling; and (iv) $p(s-a, s)$, the probability that an individual that was shoaling and is currently alone will begin to shoal. The probabilities of maintaining one's current state can be calculated from their respective transition probability-e.g., $p(a-s, a)+p(a-s, s)=$ 1. 
probabilities, data were pooled across all focal follows within each group. Transition probabilities were then estimated for each group as simple proportions from the observed sequences of shoaling behavior. Weighted mean transition probabilities were calculated for each treatment, where weighting was based on the relative frequency of opportunities for state transitions within a group.

Model goodness-of-fit was assessed by comparing the observed distributions of the durations of social contact and of being alone with simulated distributions generated using the weighted mean transition probabilities estimated for each treatment. Each simulated run maintained the observed starting state and four-minute observation time for each focal follow; $10^{4}$ simulations were conducted for each treatment. These comparisons revealed that first-order Markov chain models provided a poor fit to the observed data, in that many observed values fell outside the $2.5 \%$ and $97.5 \%$ percentiles generated by the simulations. Therefore, I constructed second-order Markov chain models for each treatment in which the probability of transitioning between states is determined jointly by an individual's state at times $t$ and $t-1$.

The second-order model is characterized by the probabilities of an individual beginning to shoal after having been alone for two consecutive time points [ $p(a-a, s)$ ], of ceasing to shoal after having been shoaling for two consecutive time points $[p(s-s, a)]$, of starting to shoal after having been shoaling at time $t-1$ and alone at time $t[p(s-a, s)]$, and of ceasing to shoal after having been alone at time $t-1$ and shoaling at time $t[p(a-s$, a)] (Figure 5.2b). As above, transition probabilities were calculated for each group using data pooled across all focal follows, and weighted mean transition probabilities were calculated for each treatment. Goodness-of-fit was assessed as described above, with 
each simulated run now maintaining the initial two states observed for each focal follow. Nonparametric bootstrapping — generating $10^{4}$ simulated datasets—-was used to obtain 95\% confidence intervals for the weighted mean transition probabilities using the boot package (Davison \& Hinkley, 1997; Canty \& Ripley, 2016) in R version 3.3.1 (R Core Team, 2016).

\subsubsection{Nearest Neighbor Preferences}

To test whether fish expressed non-random nearest neighbor preferences, association strength was quantified between each pair in terms of the average duration of a contact phase between them and their total number of shared contact phases. A contact phase between two individuals was defined as one or more consecutive observations in which one of the individuals was recorded as being the nearest neighbor of the other. I then employed randomization tests where the observed number of contact phases and their durations were maintained for each individual, but nearest neighbor identities were randomized prior to recalculating association strengths using both methods described above. This procedure was conducted $10^{4}$ times per group. The test statistic was the sum of squares of the association strength across all dyads. If the observed test statistic was in the top $5 \%$ of the distribution generated by the randomization procedure, this was used as evidence that focal fish were expressing individual-specific nearest neighbor preferences. Combined $P$ values for a treatment were obtained using Fisher's omnibus procedure (Haccou \& Meelis, 1994).

\subsubsection{Phenotypic Assortment}

Using weighted association networks, where edge weights equaled the number of shared contact phases between each pair (see Results), I obtained weighted assortativity 
coefficients $\left(r^{w}\right)$ in terms of activity level, shoaling tendency, and body length for each group using the assortnet package (Farine, 2014) in R version 3.3.1 (R Core Team, 2016). Positive values of $r^{w}$ indicate that phenotypically similar individuals tend to associate, while negative values indicate that individuals associate more often with phenotypically dissimilar group mates. Assortativity coefficients and standard errors for each phenotypic trait were averaged across each network within a treatment. To assess whether mean $r^{w}$ for a given phenotypic trait significantly differed between treatments, $10^{4}$ randomized networks were generated for each group as described above. For each of the $10^{4}$ sets of randomized networks, the difference in mean $r^{w}$ between treatments was obtained. I then compared the observed treatment difference in mean $r^{w}$ to the distribution of randomized treatment differences. If the observed value placed in the upper or lower $2.5 \%$ of the distribution, this was used as evidence of a statistically significant difference between treatments in patterns of assortment.

\subsubsection{Network-Based Diffusion Analysis (NBDA)}

To examine whether perceived ambient predation risk affected the probability that a group would solve the foraging task, I fit a binomial generalized linear model. Treatment, experimental block, and their interaction were included as explanatory variables, while the response was whether or not at least one individual in a group had successfully solved the foraging task. Statistical significance was assessed using likelihood ratio tests. Analyses were conducted in R version 3.3.1 (R Core Team, 2016).

I used network-based diffusion analysis (NBDA) — specifically the time-ofacquisition diffusion analysis (TADA) variant - to examine the rates and patterns of information diffusion within each treatment (Franz \& Nunn, 2009; Hoppitt \& Laland, 
2013). NBDA infers social transmission if the rate at which naïve individuals acquire novel information — or adopt a novel behavioral trait — increases with the strength of their connectedness to informed individuals within their group. An individual's latency to first enter the foraging device was used to indicate when it became informed regarding the location of food and how to access it. A general form of the NBDA model, extended to consider diffusions in separate groups, is given by:

$$
\lambda_{i k}(t)=\lambda_{0}(t)\left(1-z_{i k}(t)\right)\left(s \sum_{j=1}^{N_{k}} a_{i j k} z_{j k}(t)+1\right)
$$

in which $\lambda_{i k}(t)$ is the rate at which individual $i$ in group $k$ acquires the task solution at time $t, \lambda_{0}$ is the baseline (asocial) rate of acquisition, $z_{i k}(t)$ is the informational status of individual $i$ in group $k$ at time $t$ where $z=1$ is informed and $z=0$ is naïve, $s$ is the fitted social transmission parameter, and $a_{i j k}$ is the association strength between individuals $i$ and $j$ in group $k$ (see Hoppitt \& Laland, 2013 for additional details). The rate of social transmission from an informed to a naïve individual is assumed to be linearly proportional to the strength of network connection between them, $a_{i j}$, and scaled by $s$.

Significantly more low-risk groups solved the foraging task than high-risk groups (see Results), so separate TADAs were carried out for each treatment. I examined the explanatory power of two network types representing potential transmission pathways: (i) association networks, in which the association strength between each pair equaled their total number of shared contact phases, and (ii) homogeneous networks, in which the association strength between each pair was set to 1. For high-risk groups, I also included contact-duration networks in which the association strength between group mates equaled the mean duration of a contact phase between them (see Results). Inclusion of the 
association and contact-duration networks tested whether the rate of social transmission of the task solution was proportional to the strength of association between individuals, while inclusion of the homogeneous networks tested whether social transmission operated independently of the social networks. Social transmission refers to instances where the behavior of informed individuals facilitates information acquisition by naïve group members (Hoppitt \& Laland, 2013). In order to avoid counting simultaneous discovery of the task solution as instances of information transfer, I did not allow social transmission between individuals that solved the task within 10 seconds of one another (Atton et al., 2012; Farine, Aplin, et al., 2015).

Body length and experimental block were included as individual-level variables to assess their influence on an individual's baseline solving rate. Body length was standardized to zero mean and unit variance. For the TADA for the high-risk groups, block was not included as all three groups included in the analysis originated from the same block. Both additive and multiplicative TADA variants were considered (Hoppitt, Boogert et al., 2010). In the additive variant, individual-level variables affect only an individual's baseline solving rate-i.e., how rapidly does an individual acquire information on its own. In the multiplicative variant, these variables also affect social transmission, such that if individual $\mathrm{A}$ has a baseline solving rate twice that of individual B, individual A's total solving rate will remain twice that of B's as long as both are equally connected to informed individuals. Finally, models that allowed the baseline solving rate to systematically increase (or decrease) over time were compared with those that constrained it to be constant. 
I adopted an information-theoretic approach for the TADA (Burnham \& Anderson, 2002). Models were fitted for every combination of network type, individuallevel variable(s), TADA variant, and baseline rate function. The model sets further included models in which only asocial learning was permitted $(s=0)$; within these models, I also considered models in which baseline solving rates could vary between groups as an alternative explanation to social transmission for between-group differences in solving rates. Akaike weights based on AICc were obtained for each fitted model. The top-ranked TADA model for the low-risk groups accounted for $96.8 \%$ of the total Akaike weights; as such, the results from this model alone are presented. With regards to the TADA for the high-risk groups, the two highest-ranked models accounted for $99.1 \%$ of the total Akaike weight. Model-averaging was therefore conducted over these two models as described in Burnham and Anderson (2002).

Confidence intervals for parameter estimates were computed using profile likelihood techniques (Morgan, 2009); in the case of the TADA for the high-risk groups, confidence intervals were conditional on the top-ranked model and adjusted to account for model selection uncertainty (Burnham \& Anderson, 2002). Standard errors were obtained using a numerical estimate of the Hessian matrix via the optim function in $\mathrm{R}$ version 3.3.1 (Morgan, 2009; R Core Team, 2016).

The estimated probability that individual $i$ solved the foraging task via social transmission is given by:

$$
s \sum_{j=1}^{N} a_{i j} z_{j}\left(t_{i}\right) /\left(s \sum_{j=1}^{N} a_{i j} z_{j}\left(t_{i}\right)+1\right)
$$


where $t_{i}$ is the time at which $i$ solved the task. Where social transmission of the task solution was supported, this value was averaged across all individuals who solved the task within a treatment. NBDA was conducted in R version 3.3.1 (R Core Team, 2016) using code (version 1.2.11) freely available at http://lalandlab.st-andrews.ac.uk/freeware. 2.4.6 Movement through the Control Patch

To examine whether perceived ambient predation risk influenced movement within the arena following introduction of the novel foraging task, I compared between treatments: (i) the number of individuals within each group who entered an arbitrary space within the arena—-hereafter, the control patch — and (ii) the standard deviation of their latencies to do so. High levels of activity should result in more fish entering the control patch, while less variability in initial entry times is suggestive of more cohesive shoaling behavior. Due to overdispersion, the total number of individuals within each group to pass through the control patch at least once was analyzed using a negative binomial generalized linear model. The standard deviation of individual latencies to first enter the patch, weighted by the number of group members who entered it at least once, was analyzed using a linear model. For this latter model, only groups in which at least two individuals entered the control patch could be included; 13 low-risk and 10 high-risk groups met this criterion. Treatment, experimental block, and their interaction were included as explanatory variables. Statistical significance was assessed using likelihood ratio tests. Analyses were carried out in R version 3.3.1 (R Core Team, 2016).

To further explore movement patterns following the task introduction, an additional TADA was conducted using the latency to first enter the control patch as the trait of interest. Here, a positive result for $s$ suggests that individuals were in association 
with others who had recently passed through the patch when first entering it themselves. As co-arrivals at the control patch were of interest, "transmission" was permitted between any pair of individuals, regardless of the length of time between their entries. The TADA was conducted as those described above, with the following exceptions. First, an individual-level variable which allowed for the baseline rate to vary between treatments was included. Second, two social transmission parameterizations were considered: (i) models that permitted the strength of "transmission" to vary between treatments, and (ii) models that assumed "transmission" strength was equal across all experimental groups. Finally, as the contact-duration networks received essentially no support in the low-risk groups (see Results), only the association and homogeneous networks were included here.

\section{Results}

\subsection{Pilot Work}

Pre-injection behavior did not vary based on treatment $\left(\mathrm{F}_{3,12}=0.75, P=0.543\right)$, meaning that behavioral differences between the pre- and post-injection periods could be examined directly. There was a significant treatment effect on behavioral responses to the stimulus presentation relative to behavior observed prior to it $\left(\mathrm{F}_{3,12}=8.59, P=0.003\right)$. Groups exposed to conspecific alarm cues significantly reduced their activity level $\left(\mathrm{F}_{1,14}\right.$ $=23.2, P<0.001)$ and spent more time near the substrate $\left(\mathrm{F}_{1,14}=7.85, P=0.014\right)$ when compared to groups exposed to water (Figure 5.3). Groups exposed to alarm cues also exhibited a non-significant increase in shoaling behavior relative to water-exposed groups $\left(\mathrm{F}_{1,14}=4.49, P=0.052\right.$; Figure 5.3). Reduced activity, staying lower within the water column, and exhibiting increased shoaling cohesion are all characteristic anti-predator 


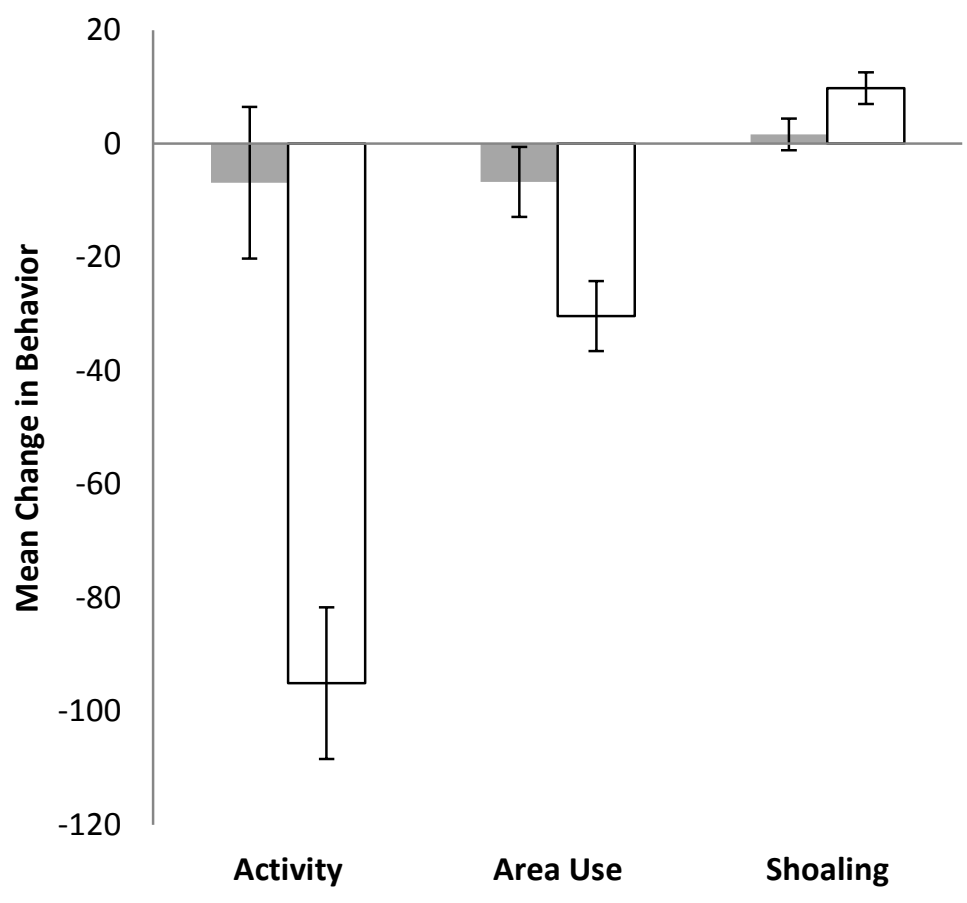

Figure 5.3 Mean ( \pm SE) change in behavior from the pre-injection to the post-injection period for groups exposed to water (grey bars) and to conspecific alarm cues (white bars). A reduction in activity level, occupying areas lower within the water column, and increased shoaling behavior are characteristic anti-predator responses in guppies. $n=8$ groups per treatment. 
responses in guppies and other fish species (Brown et al., 2009; Brown et al., 2013; Brown et al., 2015), indicating that our captive population expressed species-typical responses to the threat of predation.

\subsection{Markov Chain Fission-Fusion Models}

Comparison of the observed distributions of the durations of social contact and of being alone with simulated distributions generated from the weighted mean transition probabilities estimated for each treatment indicated that first-order Markov chain models provided a poor fit to the observed data. Modeling shoaling behavior using second-order Markov chains, in which transition probabilities are defined jointly by behavior at times $t$ -1 and $t$, better reflected the observed fission-fusion dynamics-i.e., the majority of observed values fell within the $2.5 \%$ and $97.5 \%$ percentiles generated by the simulations (Figure 5.4). This suggests that shoaling decisions were influenced by both an individual's current and recent behavior. While some deviations from the models' predictions were present, the predicted percentile ranges were conservative in that the simulated distributions did not account for uncertainty in the transition probability estimates. Weighted mean transition probabilities did not vary between treatments, suggesting that repeated exposure to conspecific alarm cues did not influence guppies' fission-fusion behavior relative to that of low-risk groups in the absence of an acute threat (Figure 5.5).

\subsection{Nearest Neighbor Preferences}

Nearest neighbor associations in low-risk groups exhibited non-random structure in terms of the number of shared contact phases between group mates (Omnibus test: $\mathrm{X}^{2}$ $=182.43, \mathrm{df}=32, P<0.001$ ), but not in terms of the mean duration of contact (Omnibus 

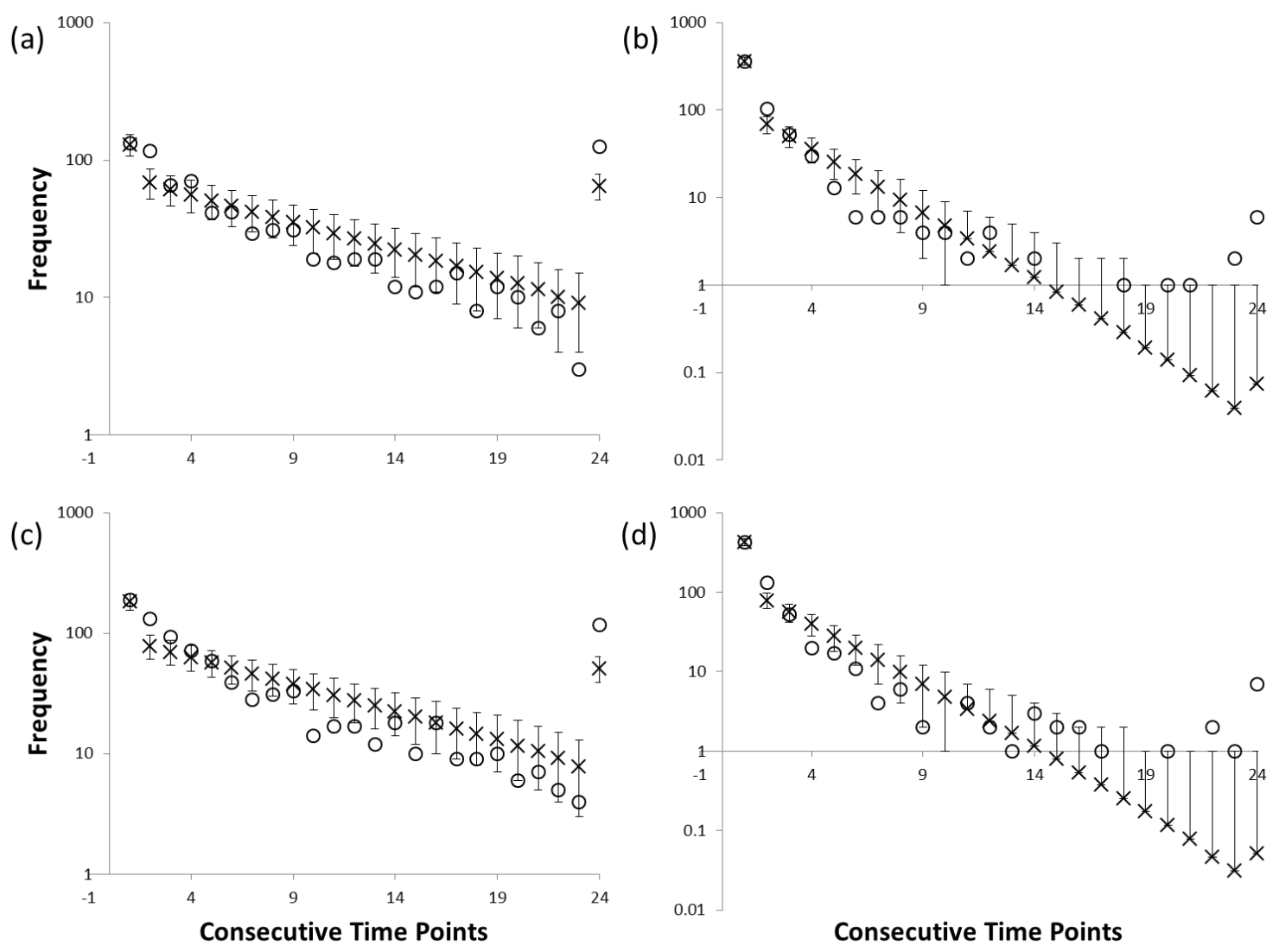

Figure 5.4 Frequency distributions of (a) the durations of continuous social contact and (b) the durations of time spent alone for low-risk groups ( $n=16$ groups). Observed data are depicted as circles. Using weighted mean transition probabilities, $10^{4}$ simulations of the second-order Markov chain model were generated. The mean values ( $x$ 's) and the $2.5 \%$ and $97.5 \%$ percentiles for each phase length obtained from these simulations are depicted. Values of 0 cannot be displayed on a logarithmic plot and are omitted. For each simulated run, the initial two states observed for each focal individual and the fourminute observation time per focal follow were maintained. The corresponding data for high-risk groups ( $n=16$ groups) are depicted in (c) \& (d). 


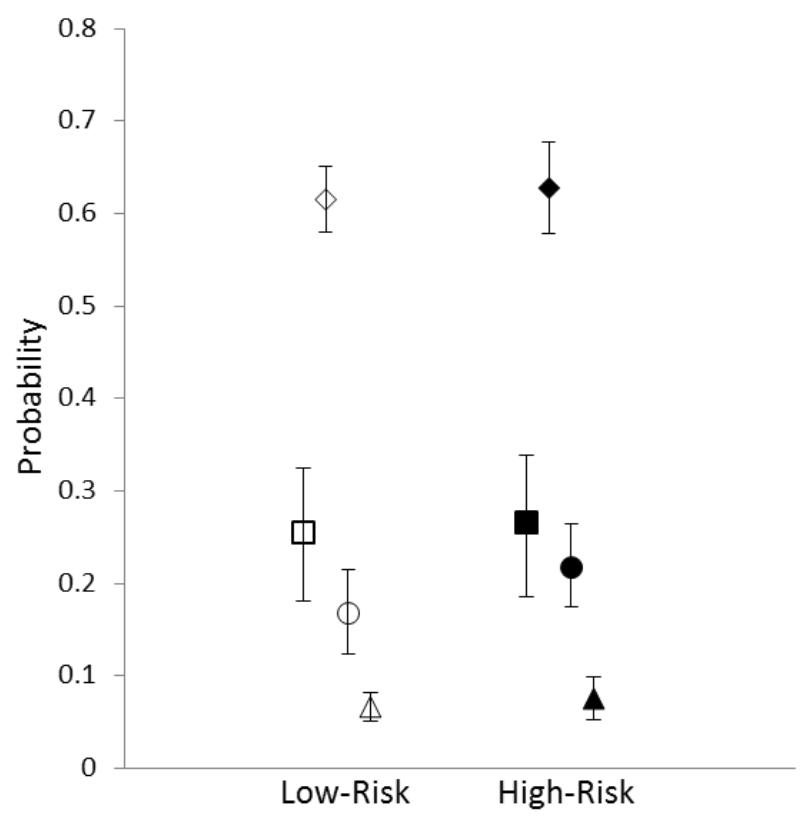

Figure 5.5 Weighted mean transition probabilities and 95\% confidence intervals for the second-order Markov chain models. Diamonds represent $p(s-a, s)$, squares represent $p(a$ $a, s)$, circles represent $p(a-s, a)$, and triangles represent $p(s-s, a)$. Transition probabilities for the high-risk treatment are denoted by filled shapes and for the low-risk treatment by open shapes; $n=16$ groups per treatment. 
test: $\left.\mathrm{X}^{2}=31.43, \mathrm{df}=32, P=0.495\right)$. In high-risk groups, nearest neighbor associations exhibited non-random structure in terms of both the number of shared contact phases (Omnibus test: $\mathrm{X}^{2}=194.79$, $\mathrm{df}=32, P<0.001$ ) and their mean duration (Omnibus test: $\left.X^{2}=69.28, \mathrm{df}=32, P<0.001\right)$.

\subsection{Phenotypic Assortment}

There was no difference between low- and high-risk groups with regards to assortment by activity level $\left(10^{4}\right.$ randomizations, 2 -tailed $\left.P=0.455\right)$. On average, lowrisk groups were significantly more disassorted than high-risk groups with regards to both shoaling tendency $\left(10^{4}\right.$ randomizations, 2-tailed $\left.P=0.013\right)$ and body length $\left(10^{4}\right.$ randomizations, 2 -tailed $P<0.001)$. Mean assortativity coefficients and standard errors for each treatment are provided in Table 5.1.

\subsection{Network-based Diffusion Analysis (NBDA)}

The interaction between background predation risk and experimental block did not influence the probability that at least one individual within a group would successfully solve the device and was removed from the model $\left(\mathrm{X}^{2}=4.81, \mathrm{df}=3, P=\right.$ 0.187). High-risk groups were significantly less likely to solve the foraging task $\left(X^{2}=\right.$ $8.90, \mathrm{df}=1, P=0.003$ ). While 10 out of 16 low-risk groups contained at least one individual that solved the task, the same was true in only three out of 16 high-risk groups. In addition, experimental block had a significant effect on a group's solving probability $\left(\mathrm{X}^{2}=9.31, \mathrm{df}=3, P=0.025\right)$. Parameter estimates, standard errors, and $95 \%$ confidence intervals are provided in Table 5.2.

To be included in the TADA, a group needed to contain at least two individuals who solved the task, resulting in nine low-risk and three high-risk groups. Out of the set 
Table 5.1 Mean assortativity coefficients $\left(r^{w}\right)$ and standard errors

\begin{tabular}{lcc}
\hline & $r^{w}$ & SE \\
\hline Low-risk groups & -0.10 & 0.12 \\
Activity level & -0.12 & 0.11 \\
Bhoaling tendency & -0.17 & 0.12 \\
\hline High-risk groups & & 0.12 \\
\hline Activity level & -0.10 & 0.10 \\
Shoaling tendency & -0.08 & 0.12 \\
Body length $(\mathrm{cm})$ & -0.10 & \\
\hline
\end{tabular}

Table 5.2 Parameter estimates, standard errors, and 95\% confidence intervals for the binomial generalized linear model examining the probability of a group solving the foraging $\operatorname{task}^{a}$

\begin{tabular}{lccc}
\hline & Estimate & SE & $95 \%$ CI \\
\hline Intercept & -4.09 & 1.58 & $-7.91,-1.51$ \\
Low background risk & 2.90 & 1.20 & $0.90,5.98$ \\
Block B & 1.83 & 1.46 & $-0.84,5.25$ \\
Block C & 4.27 & 1.73 & $1.34,8.34$ \\
Block D & 1.83 & 1.46 & $-0.84,5.25$ \\
\hline
\end{tabular}

$a$ : Estimates were obtained from the model in which the interaction between treatment and experimental block had been removed; the interaction did not have a significant effect on the response variable. The high-risk treatment and experimental block A served as the overall baseline. $n=16$ groups per treatment. 
of 44 fitted models for the low-risk groups, one model received $96.8 \%$ of the summed Akaike weights. As such, only the results of this top-ranked model are presented (Table 5.3). Social transmission of the foraging task solution was strongly supported in the lowrisk groups, though the top-ranked model included the homogeneous networks. This suggests that the diffusion of the task solution did not follow the pattern of connections within the association networks. For each informed group member, a naïve individual's solving rate increased by $s=1.03(95 \% \mathrm{CI}: 0.72,1.44)$ relative to the average asocial solving rate, corresponding to an estimated $48.5-58.8 \%$ of the 68 solving events occurring as a result of social transmission (Table 5.3). The top model used the multiplicative TADA variant, meaning that individual-level variables affected both an individual's baseline solving rate and social transmission. Larger fish were slower to solve the task, while solving rates varied across the experimental blocks (Table 5.3). A shape parameter $>1$ suggests that the baseline rate of solving systematically increased over time (Table 5.3).

With regards to the three high-risk groups, out of the set of 26 fitted models, the top two models accounted for $99.1 \%$ of the total Akaike weights. As such, modelaveraging was conducted over these two models. Neither of the top models supported an effect of social transmission of the task solution $(s=0)$, suggesting that the 15 solving events in the high-risk groups occurred primarily through individual learning (Table 5.4). In general, larger individuals were faster to solve the task in the high-risk groups, though wide confidence intervals suggest that the effect of body length on solving rate was inconsistent (Table 5.4). Additionally, baseline solving rates were found to vary between groups and systematically increased over time (Table 5.4). 
Table 5.3 TADA parameter estimates, standard errors, and 95\% confidence intervals for the low-risk groups ${ }^{a}$

\begin{tabular}{|c|c|c|c|}
\hline & Estimate & SE & $95 \% \mathrm{CI}$ \\
\hline Social transmission strength, $s$ & 1.03 & 0.18 & $0.72,1.44$ \\
\hline Individual-level variables $^{b}$ & & & \\
\hline Body length $(\mathrm{cm})$ & -0.23 & 0.06 & $-0.35,-0.11$ \\
\hline Block B & -0.62 & 0.18 & $-0.97,-0.26$ \\
\hline Block C & -0.35 & 0.17 & $-0.68,-0.02$ \\
\hline Block D & 0.21 & 0.18 & $-0.14,0.57$ \\
\hline
\end{tabular}

$a$ : Inclusion of the homogeneous networks assumes an equal rate of social transmission between any pair of group mates. The shape parameter was 2.72 , indicating a baseline solving rate that systematically increased over time. The top model used the multiplicative TADA variant, meaning that the individual-level variables affected both an individual's baseline solving rate and social transmission rate.

$b$ : Parameter estimates for the individual-level variables provide the additive effect on the baseline solving rate on the log scale for an increase in one unit for that variable. To obtain an individual-level variable's multiplicative effect on the solving rate, it must be back-transformed. For example, if individual A is $2 \mathrm{~cm}$ longer than individual B, A will solve the task $\exp (-0.23 \times 2)=0.63$ times slower than $\mathrm{B}$. The baseline solving rate for block A was used as the overall baseline-i.e., it equaled 0 . 
Table 5.4 Model-averaged TADA parameter estimates, standard errors, and 95\% confidence intervals for the high-risk groups ${ }^{a}$

\begin{tabular}{lccc}
\hline Individual-level variables $^{b}$ & Estimate & SE & $95 \%$ CI \\
\hline Body length (cm) & 0.29 & 0.17 & $-0.28,0.78$ \\
Group B & -0.84 & 0.20 & $-1.61,-0.26$ \\
Group C & 0.22 & 0.29 & $-0.68,1.09$ \\
\hline
\end{tabular}

$a$ : The shape parameter was 2.49 , indicating a baseline solving rate that systematically increased over time. Model-averaging was conducted over the top two models; neither model supported an effect of social transmission of the task solution $(s=0)$.

$b$ : Interpretation of the individual-level variables and calculation of their multiplicative effect on the solving rate is as described above in Table 5.3. The baseline solving rate for group A was used as the overall baseline-i.e., it equaled 0. 
Table 5.5 Parameter estimates, standard errors, and 95\% confidence intervals for models examining movement through the control patch following introduction of the foraging $\operatorname{task}^{a}$

\begin{tabular}{lccc}
\hline & Estimate & SE & 95\% CI \\
\hline Number of first entries (Neg. bin. linear model) & & & \\
\cline { 1 - 3 } Intercept & -1.20 & 0.35 & $-1.89,-0.50$ \\
Low background risk & 0.40 & 0.29 & $-0.17,0.98$ \\
Block B & 0.50 & 0.42 & $-0.33,1.34$ \\
Block C & 0.51 & 0.42 & $-0.32,1.35$ \\
Block D & 0.56 & 0.42 & $-0.27,1.39$ \\
Negative binomial dispersion parameter & 2.25 & 1.13 & \\
\hline Standard deviation of entry times (linear model) & & & \\
\hline Intercept & 342.47 & 57.74 & $229.30,455.64$ \\
Low background risk & -102.76 & 42.25 & $-185.58,-19.94$ \\
Block B & -147.87 & 64.0 & $-273.30,-22.44$ \\
Block C & -66.34 & 64.61 & $-192.97,60.29$ \\
Block D & -143.82 & 63.40 & $-268.07,-19.57$ \\
\hline
\end{tabular}

$a$ : Estimates were obtained from the model in which the interaction between treatment and experimental block had been removed; the interaction did not have a significant effect on either response variable. The high-risk treatment and experimental block A served as the overall baseline. For the model examining the number of first entries into the control patch per group, $n=16$ groups per treatment. For the model examining the standard deviation in times of arrival at the control patch, $n=13$ low-risk groups and 10 high-risk groups. 
Table 5.6 Model-averaged parameter estimates, standard errors, and 95\% confidence intervals for the TADA examining movement through the control patch ${ }^{a}$

\begin{tabular}{|c|c|c|c|}
\hline & Estimate & SE & $95 \% \mathrm{CI}$ \\
\hline \multicolumn{4}{|l|}{ Social influences on arrival times, $s$} \\
\hline Low-risk groups & 0.25 & 0.03 & $0.21,0.32$ \\
\hline High-risk groups & 0.13 & 0.02 & $0.10,0.17$ \\
\hline \multicolumn{4}{|l|}{ Individual-level variables $^{b}$} \\
\hline High-risk groups' baseline arrival rate & -0.02 & 0.06 & $-0.18,0.05$ \\
\hline Body length $(\mathrm{cm})$ & -0.03 & 0.05 & $-0.16,0.02$ \\
\hline Block B & 0.06 & 0.13 & $-0.12,0.39$ \\
\hline Block C & 0.14 & 0.13 & $-0.04,0.46$ \\
\hline Block D & 0.29 & 0.12 & $0.12,0.58$ \\
\hline \multicolumn{4}{|c|}{$a$ : Inclusion of the association networks suggests that individuals were greatly influenced } \\
\hline \multicolumn{4}{|c|}{ rate of arrival at the control patch was constant over time. The multiplicative TADA } \\
\hline \multicolumn{4}{|c|}{ variant was strongly favored, meaning that the individual-level variables affected both an } \\
\hline \multicolumn{4}{|c|}{ individual's baseline rate of arrival and social influences over its arrival rate. } \\
\hline \multicolumn{4}{|c|}{$b$ : Interpretation of the individual-level variables and calculation of their multiplicative } \\
\hline effect on the arrival rate is as described & Table 5 . & e bas & te of arrival \\
\hline
\end{tabular}




\subsection{Movement through the Control Patch}

The interaction between treatment and experimental block did not influence the number of individuals within a group that entered the control patch at least once during the foraging task $\left(\mathrm{X}^{2}=0.60, \mathrm{df}=3, P=0.896\right)$ and was removed from the model to permit testing of main effects. Neither the background level of predation risk experienced by a group $\left(\mathrm{X}^{2}=1.80, \mathrm{df}=1, P=0.180\right)$ nor their experimental block $\left(\mathrm{X}^{2}=2.07, \mathrm{df}=3\right.$, $P=0.557)$ influenced how many fish entered the control patch. With regards to the standard deviation of entry times within a group, there was no effect of the interaction between treatment and experimental block $\left(\mathrm{X}^{2}=0.72, \mathrm{df}=3, P=0.869\right)$; as such, it was removed from the model. Individuals in low-risk groups exhibited less variation in their latency to initially enter the control patch $\left(\mathrm{X}^{2}=5.91, \mathrm{df}=1, P=0.015\right)$, which is suggestive of more cohesive shoaling behavior. Experimental block had a nearsignificant effect on variation in entry times $\left(\mathrm{X}^{2}=7.44, \mathrm{df}=3, P=0.059\right)$. Parameter estimates, standard errors, and 95\% confidence intervals for the above models are presented in Table 5.5.

The TADA examining movement through the control patch included all groups in which at least two individuals entered the patch, resulting in 13 low-risk and 10 high-risk groups being included. Models which included social influences over patch arrival times (i.e., $s>0$ ) were strongly favored ( $>99.9 \%$ total Akaike weights) relative to models which considered only asocial factors impacting movement. Based on Akaike weights, there was strong support for the multiplicative TADA model (95.9\% support), inclusion of the association networks (> 99.9\% support), and a constant baseline rate of arrival 
(72.0\% support). Model-averaging was conducted over the 16 models that met all of these criteria, accounting for $69.3 \%$ of the total Akaike weights.

Inclusion of the association networks suggests that individuals were more likely to arrive at the patch if group mates with whom they were strongly connected had already done so. Social influences on patch arrival times were detected in both treatments (Table 5.6). However, this effect was stronger in low-risk groups relative to high-risk ones, with the difference estimated at $s=0.12$ (95\% CI: $0.09,0.16)$; this may indicate that individuals in low-risk groups tended to travel in larger and/or more cohesive shoals following the introduction of the foraging task relative to high-risk groups. Use of the multiplicative TADA variant means that individual-level variables impacted both the baseline rate of arrival and social influences on arrival times. There was no effect of risk treatment or body length on an individual's arrival rate, but arrival rates varied across experimental blocks (Table 5.6).

\section{Discussion}

The threat of predation is a powerful selective agent that varies across space and time (Lima \& Bednekoff, 1999; Stankowich \& Blumstein, 2005). Here, I demonstrated experimentally that variation in background levels of predation risk can shape fine-scale patterns of association while leaving the general fission-fusion processes from which social networks emerge unaltered. In addition, heightened perception of ambient predation risk inhibited the uptake and subsequent transmission of novel foraging information, possibly by increasing the perceived costs of individual learning and/or inducing heightened neophobic responses. Thus, while phenotypically plastic antipredator behavior can facilitate recognition of - and appropriate responses to - a diverse 
array of threats (Brown et al., 2013), it can also incur opportunity costs and potentially impact access to information relevant to decision-making in a range of contexts (Danchin et al., 2004; Valone, 2007).

Fission-fusion behavior can facilitate rapid responses to spatiotemporal variation in the environment through dynamic changes in group size and composition (Aureli et al., 2008; Kelley et al., 2011; Voelkl et al., 2016). However, my results indicate that variation in perceived ambient predation risk did not impact individuals' decision-making regarding whether to leave or join a shoal. As the rate of movement between groups is predicted to influence how rapidly information (or disease) spreads through a population (Aplin et al., 2012; Krause et al., 2016), this may suggest that, in the absence of an acute threat, similar diffusion dynamics are at work in populations exposed to different levels of predation risk.

Recent work suggests that wild guppies actively combat changes in their fissionfusion behavior, which led that study's authors to propose that these dynamics may represent an evolved defense that prevents predators from predicting periods in which prey individuals are likely to be alone and vulnerable to attack (Wilson et al., 2015). Still, given the greater vulnerability of lone individuals to predation (Krause \& Ruxton, 2002), it remains surprising that guppies exposed to high-risk conditions were no more likely to join a shoal when alone than were those exposed to low-risk conditions. Despite the robustness of these social dynamics to changes in population density (Wilson et al., 2015), it may be that the comparatively high densities within the arenas masked treatment differences in behavior. Nevertheless, my findings suggest that variation in fission-fusion processes is unnecessary for shifts in social network structure to occur. 
Social networks within both treatments tended to be disassortative, though highrisk groups were less disassorted with regards to shoaling tendency and body length than were low-risk groups. This may suggest a tradeoff between the potential benefits of association with unlike individuals, such as competitive advantages or greater foraging efficiency (Metcalfe \& Thompson, 1995; Dyer et al., 2009; Aplin et al., 2014), and phenotypic assortment when faced with the threat of predation. Selective targeting of phenotypically odd individuals by predators can favor within-group homogeneity in prey species (Landeau \& Terborgh, 1986; Krakauer, 1995; Rodgers et al., 2015), thereby promoting positive assortment between phenotypically similar individuals. Within fish, size-assortative grouping has been particularly well-studied, and is commonly observed in response to an elevated threat of predation (Krause \& Ruxton, 2002).

Ambient predation risk also shaped the expression of social preferences. Within low-risk groups, non-random patterns of association were detected solely in terms of contact frequency, while nearest neighbor preferences within high-risk groups were expressed through both contact frequency and the mean duration of contact. Recent work on wild populations suggests that high levels of predation risk can promote the formation of stable social bonds (Kelley et al., 2011; Heathcote et al., 2017). Similarly, individuals that tend to be more risk-averse are more likely to maintain temporally stable associations with group mates across a range of timescales relative to more risk-prone individuals (Pike et al., 2008; Aplin et al., 2013). Such strong, long-lasting associations can facilitate enhanced coordination during anti-predator behavior, and the maintenance of cooperative relationships (Chivers et al., 1995; Micheletta et al., 2012). For example, stable bonds between wild female guppies were predictive of increased cooperation during inspection 
of potential predators - e.g., females exchanged the risky lead position during an inspection bout more frequently (Croft et al., 2006). Taken together, these results are consistent with changes in assortment and temporal patterns of association representing behavioral responses to a heightened perception of risk.

Exposure to high background predation risk also inhibited the acquisition of novel foraging information, in that high-risk groups were less likely than low-risk groups to solve the foraging task. This result may have stemmed from high-risk conditions promoting stronger neophobic responses towards the novel task (Greenberg, 2003; Brown et al., 2013). In prey populations exposed to intense predation, heightened neophobia reduces learning costs when dealing with potential threats - that is, neophobic individuals are less likely to mistakenly classify a novel predator as benign (Brown et al., 2013). Conversely, weaker neophobic responses in low-risk environments help individuals to avoid wasting time and energy on unnecessary anti-predator behavior (Lima \& Dill, 1990). While the benefits of neophobia in dangerous environments can be substantial, the failure of high-risk groups to exploit a novel foraging opportunity demonstrates a potential cost. Similar findings have been reported in wild guppies in which heightened spatial neophobia in populations exposed to high levels of predation risk resulted in decreased foraging success (Elvidge et al., 2016). Consistent with this interpretation, the diffusion analyses for the foraging task supported a baseline solving rate that increased over time, which is suggestive of neophobic responses that eventually waned in those groups that successfully solved the task (Hoppitt, Kandler, et al., 2010).

A non-mutually exclusive alternative is that the lower probability of solving the task in high-risk groups resulted from higher costs of engaging in personal sampling of 
the environment (Laland, 2004; Webster \& Laland, 2008). Entering the device could entail losing visual contact with group mates outside, thus depriving an individual of the informational benefits of grouping and increasing its vulnerability to predation (Krause \& Ruxton, 2002; Rosenthal et al., 2015). Even if the device itself was not perceived as especially threatening, individuals in high-risk groups may have been less likely to investigate it if such exploration necessitated a reduction in vigilance efforts (Lima \& Dill, 1990). My results suggest that shoals in high-risk groups tended to be smaller and/or less cohesive than those in low-risk groups following the introduction of the foraging task. As such, the perceived costs of reduced vigilance may have been especially pronounced in high-risk groups, given the positive relationship between group size and predator detection ability (Pulliam, 1973).

Likewise, if individuals in low-risk groups tended to approach the foraging task as part of a larger, more cohesive shoal, this could promote both asocial acquisition and social transmission of the task solution. By lowering individual predation risk through dilution effects and enhanced predator detection ability (Pulliam, 1973; Krause \& Ruxton, 2002), larger group sizes can facilitate exploration of potentially threatening stimuli and novel environments (Dugatkin, 1991; Croft et al., 2006; Brown \& Irving, 2014). Such social facilitation may have contributed to the higher solving probability experienced by low-risk groups (Zajonc, 1965; Hoppitt \& Laland, 2013).

Larger group sizes can also promote the rapid diffusion of social information (Day et al., 2001). Social transmission of the task solution was strongly supported in lowrisk groups, and was responsible in part for an estimated 54\% of solving events. Social transmission of foraging information in fish shoals can occur through relatively simple 
social learning mechanisms, such as local enhancement (Thorpe, 1956; Hoppitt \& Laland, 2013). For instance, knowledgeable individuals can lead naïve group mates to a foraging site during collective movements (Swaney et al., 2001; Ioannou et al., 2015), while visual and olfactory cues associated with foraging can provide tip-offs to uninformed individuals (Lachlan et al., 1998; Johannesen et al., 2012). In the low-risk groups, social transmission appeared to operate independently of the association networks, which is consistent with individuals in these groups tending to approach and interact with the device as part of a large, cohesive shoal. Under such circumstances, individual variation in nearest neighbor preferences should be less important in determining an individual's opportunities to observe or interact with informed group mates (Webster et al., 2013).

Theoretical and empirical results indicate that when asocial learning is costly, individuals should prioritize social information use (Boyd \& Richerson, 1985; Mineka \& Cook, 1988; Laland, 2004; Webster \& Laland, 2008). Despite this prediction, there was little evidence of social transmission of the solution in those high-risk groups that successfully solved the foraging task. While high-risk groups may have travelled in smaller and/or less cohesive shoals relative to low-risk groups, network structure was predictive regarding their movement patterns. As such, solving events in high-risk groups may have involved co-discoveries by small numbers of strongly connected individuals. However, I recognize the highly speculative nature of attempting to draw conclusions from only three groups. Future work might provide a more direct comparison of social transmission patterns in low- and high-risk environments by seeding trained individuals 
within groups, thus providing all individuals with access to knowledgeable demonstrators.

As this study was conducted on individuals bred under captive conditions for many generations, extending these results to wild populations should be done with caution. However, my pilot study demonstrated that responses to conspecific alarm cues in our population were characteristic of anti-predator behaviors exhibited by wild-caught guppies (Brown et al., 2009). In addition, my findings are broadly consistent with prior work carried out on wild guppy populations (Brown et al., 2013; Elvidge et al., 2016; Heathcote et al., 2017). By conducting this study under controlled conditions, I provide experimental evidence that perceived ambient predation risk can influence fine-scale patterns of association, as well as the acquisition and spread of novel information. By shaping access to information, fear of predation has the potential to impact the ability of individuals to assess habitat quality (Seppänen \& Forsman, 2007), to successfully establish themselves in novel environments (Pinter-Wollman et al., 2009; Cote et al., 2010), and to locate and exploit foraging resources that are often ephemeral and difficult to find (Sundaresan et al., 2007; Aplin et al., 2012). Thus, while behavioral responses to high-risk conditions can increase the probability of survival, they can also incur opportunity costs and may affect transmission processes. 


\section{CHAPTER VI}

\section{CONCLUSION AND FUTURE DIRECTIONS}

Social network analysis provides a sophisticated framework for examining the reciprocal interplay between individual behavior and group-level structure, and to explore the ecological and evolutionary consequences of this interaction. Here, I have demonstrated the impact of social and ecological variation on the social network structure of Trinidadian guppy shoals (Poecilia reticulata), and on processes of information diffusion occurring within them. While variation in group composition and the background level of predation risk perceived by a group had little impact on the fissionfusion dynamics of shoaling, these factors were found to influence fine-scale patterns of association and phenotypic assortment. In turn, non-random patterns of association frequently predicted the diffusion of information regarding the location of foraging sites. How rapidly this information was initially acquired, and the rate at which it subsequently spread through a group, was determined by both individual- and group-level phenotypic variation, as well as by characteristics of the environment. As such, an individual's access to information critical for adaptive decision-making may frequently depend not only on its own behavior, but on the ecological and social environment in which it is embedded.

The work presented in this dissertation raises several fascinating questions for future research. Here, I highlight a few directions I believe to be especially promising. 
First, how does the collection of personal information and the transmission of social information vary based on the ecological challenges confronting individuals? For example, while bolder individuals were faster to acquire the solution to a novel foraging task, would these individuals retain this advantage when confronted with tasks in which novelty does not play a role? Prior work has suggested a possible relationship between proactive personalities - i.e., bolder, active, and more exploratory individuals — and a tendency to form inflexible routines, while shyer, more reactive individuals may be more responsive to subtle environmental changes (Marchetti \& Drent, 2000; Herborn et al., 2014). Thus, if a previously depleted foraging site becomes profitable once more, we might expect shy individuals to learn about this change more rapidly than bolder group mates.

While information flow within fish shoals can generally be explained by simple social learning mechanisms, such as social facilitation and local enhancement (Thorpe, 1956; Zajonc, 1965; Hoppitt \& Laland, 2013), the methods used in this dissertation did not allow for identification of the precise mechanisms of information transfer at work. By building networks explicitly based on such mechanisms, it should be possible to directly assess their contributions to social transmission and examine whether reliance on a particular mechanism varies based on ecological context. For example, the ability of a network based on observed leader-follower relationships to explain patterns of information flow could be compared with one constructed from simple frequencies of spatiotemporal proximity (as were primarily used here). Seeding trained individuals within groups could provide a means to assess whether key individuals play a 
disproportionate role in facilitating the diffusion of information, and to identify the potential mechanisms by which they do so (Modlmeier, Keiser, et al., 2014).

Another promising area for future study is whether certain positions within a social network - or whether certain network structures - entail trade-offs. For example, within shoals containing both familiar and unfamiliar individuals, I detected a correlation between association strength and shoaling tendency. This structural pattern may have contributed to the especially rapid diffusion of foraging information within mixed shoals, but it might also facilitate the rapid transmission of disease or parasites (Newman, 2002; Drewe \& Perkins, 2015). Similarly, high levels of connectedness in a pig-tailed macaque (Macaca nemestrina) network allowed for a diversity of partners for grooming and play behaviors, but in the absence of policing by dominant males, central network positions entailed an increased risk of conflict (Flack et al., 2006).

The extent to which individuals are capable of modifying their social network position-e.g., to shield themselves from the negative aspects of high connectedness or to increase their access to social resources - remains relatively unexplored. The evolutionary consequences of social network structure should depend in part on the nature of such responses to an individual's social environment (Farine, Montiglio, et al., 2015). Further development of statistical techniques, including stochastic actor-oriented models (Snijders et al., 2010), time-ordered networks (Blonder et al., 2012), as well as the Markov chain models used here (Wilson et al., 2014), will increasingly allow for examination of how individual social network positions and global network structures change over time and in response to ecological challenges and social perturbations. 


\section{REFERENCES}

Alcock, J. (2013). Animal behavior (10 ${ }^{\text {th }}$ ed.). Sunderland, MA: Sinauer Associates.

Alexander, R. D. (1974). The evolution of social behavior. Annual Review of Ecology and Systematics, 5, 325-383.

Allen, J., Weinrich, M., Hoppitt, W., \& Rendell, L. (2013). Network-based diffusion analysis reveals cultural transmission of lobtail feeding in humpback whales. Science, 340, 485-488.

Aplin, L. M., Farine, D. R., Mann, R. P., \& Sheldon, B. C. (2014). Individual-level personality influences social foraging and collective behaviour in wild birds. Proceedings of the Royal Society of London B: Biological Sciences, 281, 20141016.

Aplin, L. M., Farine, D. R., Morand-Ferron, J., Cockburn, A., Thornton, A., \& Sheldon, B. C. (2015). Experimentally induced innovations lead to persistent culture via conformity in wild birds. Nature, 518, 538-541.

Aplin, L. M., Farine, D. R., Morand-Ferron, J., Cole, E. F., Cockburn, A., \& Sheldon, B. C. (2013). Individual personalities predict social behaviour in wild networks of great tits (Parus major). Ecology Letters, 16, 1365-1372.

Aplin, L. M., Farine, D. R., Morand-Ferron, J., \& Sheldon, B. C. (2012). Social networks predict patch discovery in a wild population of songbirds. Proceedings of the Royal Society of London B: Biological Sciences, 279, 4199-4205.

Atton, N., Galef, B. J., Hoppitt, W., Webster, M. M., \& Laland, K. N. (2014). Familiarity affects social network structure and discovery of prey patch locations in foraging stickleback shoals. Proceedings of the Royal Society of London B: Biological Sciences, 281, 20140579.

Atton, N., Hoppitt, W., Webster, M. M., Galef, B. G., \& Laland, K. N. (2012). Information flow through threespine stickleback networks without social transmission. Proceedings of the Royal Society of London B: Biological Sciences, 279, 4272-4278. 
Aureli, F., Schaffner, C. M., Boesch, C., Bearder, S. K., Call, J., Chapman, C. A., et al. (2008). Fission-fusion dynamics: new research frameworks. Current Anthropology, 49, 627-654.

Bales, R. F. (1951). Interaction process analysis: a method for the study of small groups. Cambridge, MA: Addison-Wesley.

Barabási, A. L., \& Albert, R. (1999). Emergence of scaling in random networks. Science, 286, 509-512.

Barnard, C. J., \& Sibly, R. M. (1981). Producers and scroungers: a general model and its application to captive flocks of house sparrows. Animal Behaviour, 29, 543-550.

Barrett, L., Henzi, S. P., \& Lusseau, D. (2012). Taking sociality seriously: the structure of multi-dimensional social networks as a source of information for individuals.

Philosophical Transactions of the Royal Society of London B: Biological Sciences, 367, 2108-2118.

Bates, D., Maechler, M., Bolker, B., \& Walker, S. (2015). Fitting linear mixed-effects models using lme4. Journal of Statistical Software, 67, 1-48.

Beer, C. (1976). Some complexities in the communication behavior of gulls. Annals of the New York Academy of Sciences, 280, 413-432.

Bergmüller, R., \& Taborsky, M. (2010). Animal personality due to social niche specialisation. Trends in Ecology and Evolution, 25, 504-511.

Bigg, M. A., Olesiuk, P. F., Ellis, G. M., Ford, J. K. B., \& Balcomb, K. C. (1990). Social organization and genealogy of resident killer whales (Orcinus orca) in the coastal waters of British Columbia and Washington state. Report of the International Whaling Commission (Special Issue), 12, 383-405.

Blonder, B., \& Dornhaus, A. (2011). Time-ordered networks reveal limitations to information flow in ant colonies. PLoS One, 6, e20298.

Blonder, B., Wey, T. W., Dornhaus, A., James, R., \& Sih, A. (2012). Temporal dynamics and network analysis. Methods in Ecology and Evolution, 3, 958-972.

Blumstein, D. T., Petelle, M. B., \& Wey, T. W. (2013). Defensive and social aggression: repeatable but independent. Behavioral Ecology, 24, 457-461.

Bode, N. W. F., Franks, D. W., \& Wood, A. J. (2012). Leading from the front? Social networks in navigating groups. Behavioral Ecology and Sociobiology, 66, 835843. 
Bode, N. W. F., Wood, A. J., \& Franks, D. W. (2011a). Social networks and models for collective motion in animals. Behavioral Ecology and Sociobiology, 65, 117-130.

Bode, N. W. F., Wood, A. J., \& Franks, D. W. (2011b). The impact of social networks on animal collective motion. Animal Behaviour, 82, 29-38.

Bode, N. W. F., Wood, A. J., \& Franks, D. W. (2012). Social networks improve leaderless group navigation by facilitating long-distance communication. Current Zoology, 58, 329-341.

Boesch, C., Marchesi, P., Marchesi, N., Fruth, B., \& Joulian, F. (1994). Is nut cracking in wild chimpanzees a cultural behaviour? Journal of Human Evolution, 26, 325338.

Bolker, B. M., Brooks, M. E., Clark, C. J., Geange, S. W., Poulsen, J. R., Stevens, M. H. H., et al. (2009). Generalized linear mixed models: a practical guide for ecology and evolution. Trends in Ecology and Evolution, 24, 127-135.

Boogert, N. J., Reader, S. M., Hoppitt, W., \& Laland, K. N. (2008). The origin and spread of innovations in starlings. Animal Behaviour, 75, 1509-1518.

Borgatti, S. P., Everett, M. G., \& Freeman, L. C. (2002). UCINET 6 for Windows: software for social network analysis. Harvard, MA: Analytic Technologies.

Borgatti, S. P., Mehra, A., Brass, D. J., \& Labianca, G. (2009). Network analysis in the social sciences. Science, 323, 892-895.

Borner, K. K., Krause, S., Mehner, T., Uusi-Heikkilä, S., Ramnarine, I. W., \& Krause, J. (2015). Turbidity affects social dynamics in Trinidadian guppies. Behavioral Ecology and Sociobiology, 69, 645-651.

Boyd, R., \& Richerson, P. J. (1985). Culture and the evolutionary process. Chicago, IL: University of Chicago Press.

Bradbury, J. W., \& Vehrencamp, S. L. (2011). Principles of animal communication $\left(2^{\text {nd }}\right.$ ed.). Sunderland, MA: Sinauer Associates.

Bradbury, J. W., \& Vehrencamp, S. L. (2014). Complexity and behavioral ecology. Behavioral Ecology, 25, 435-442.

Brent, L. J. N., Lehmann, J., \& Ramos-Fernández, G. (2011). Social network analysis in the study of nonhuman primates: a historical perspective. American Journal of Primatology, 73, 720-730.

Brosset, A. (1976). Social organization in the African bat, Myotis boccagei. Zeitschrift für Tierpsychologie, 42, 50-56. 
Brown, C., \& Braithwaite, V. A. (2004). Size matters: a test of boldness in eight populations of the poeciliid Brachyraphis episcopi. Animal Behaviour, 68, 13251329.

Brown, C., \& Irving, E. (2014). Individual personality traits influence group exploration in a feral guppy population. Behavioral Ecology, 25, 95-101.

Brown, G. E., Elvidge, C. K., Ramnarine, I., Ferrari, M. C. O., \& Chivers, D. P. (2015). Background risk and recent experience influences retention of neophobic responses to predators. Behavioral Ecology and Sociobiology, 69, 737-745.

Brown, G. E., Ferrari, M. C. O., Elvidge, C. K., Ramnarine, I., \& Chivers, D. P. (2013). Phenotypically plastic neophobia: a response to variable predation risk. Proceedings of the Royal Society of London B: Biological Sciences, 280, 20122712.

Brown, G. E., Macnaughton, C. J., Elvidge, C. K., Ramnarine, I., \& Godin, J. G. J. (2009). Provenance and threat-sensitive predator avoidance patterns in wildcaught Trinidadian guppies. Behavioral Ecology and Sociobiology, 63, 699-706.

Burnham, K. P., \& Anderson, D. R. (2002). Model selection and multimodel inference: a practical information-theoretic approach ( $2^{\text {nd }}$ ed.). New York, NY: SpringerVerlag.

Burns, J. G., \& Dyer, A. G. (2008). Diversity of speed-accuracy strategies benefits social insects. Current Biology, 18, R953-R954.

Burt, R. S. (1988). The stability of American markets. American Journal of Sociology, 94, 356-395.

Canty, A., \& Ripley, B. (2016). boot: bootstrap R (S-Plus) functions. R package version 1.3-18.

Carpenter, C. R. (1942a). Characteristics of social behavior in non-human primates. Transactions of the New York Academy of Sciences: Series II, 4, 248-258.

Carpenter, C. R. (1942b). Societies of monkeys and apes. Biological Symposium, 8, 177204.

Carpenter, C. R. (1952). Social behavior of non-human primates. Structure et physiologie des sociétés animales. Colloques Internationaux du Centre National de la Recherche Scientifique, 34, 227-246.

Carter, A. J., English, S., \& Clutton-Brock, T. H. (2014). Cooperative personalities and social niche specialization in female meerkats. Journal of Evolutionary Biology, 27, 815-825. 
Carter, A. J., Marshall, H. H., Heinsohn, R., \& Cowlishaw, G. (2012). How not to measure boldness: novel object and antipredator responses are not the same in wild baboons. Animal Behaviour, 84, 603-609.

Chapman, B. B., Morrell, L. J., \& Krause, J. (2010). Unpredictability in food supply during early life influences boldness in fish. Behavioral Ecology, 21, 501-506.

Chapman, B. B., Ward, A. J. W., \& Krause, J. (2008). Schooling and learning: early social environment predicts social learning ability in the guppy, Poecilia reticulata. Animal Behaviour, 76, 923-929.

Chapple, D. G., Simmonds, S. M., \& Wong, B. B. M. (2012). Can behavioral and personality traits influence the success of unintentional species introductions? Trends in Ecology and Evolution, 27, 57-64.

Cheney, D. L. (1978a). Interactions of immature male and female baboons with adult females. Animal Behaviour, 26, 389-408.

Cheney, D. L. (1978b). The play partners of immature baboons. Animal Behaviour, 26, 1038-1050.

Chepko-Sade, B. D., Reitz, K. P., \& Sade, D. S. (1989). Sociometrics of Macaca mulatta IV: network analysis of social structure of a pre-fission group. Social Networks, $11,293-314$.

Chivers, D. P., Brown, G. E., \& Smith, R. J. F. (1995). Familiarity and shoal cohesion in fathead minnows (Pimephales promelas): implications for antipredator behaviour. Canadian Journal of Zoology, 73, 955-960.

Chivers, D. P., McCormick, M. I., Mitchell, M. D., Ramasamy, R. A., \& Ferrari, M. C. O. (2014). Background level of risk determines how prey categorize predators and non-predators. Proceedings of the Royal Society of London B: Biological Sciences, 281, 20140355.

Chivers, D. P. \& Smith, R. J. F. (1998). Chemical alarm signalling in aquatic predatorprey systems: a review and prospectus. Écoscience, 5, 338-352.

Claidière, N., Messer, E. J. E., Hoppitt, W., \& Whiten, A. (2013). Diffusion dynamics of socially learned foraging techniques in squirrel monkeys. Current Biology, 23, 1251-1255.

Clutton-Brock, T. H., Guinness, F. E., \& Albon, S. D. (1982). Red deer: behavior and ecology of two sexes. Chicago, IL: University of Chicago Press. 
Connor, R. C. (1992). Dolphin alliances and coalitions. In: A. H. Harcourt, \& F. B. M. de Waal (Eds.), Coalitions and alliances in humans and other animals (pp. 415-443). Oxford, UK: Oxford University Press.

Connor, R. C., Heithaus, M. R., \& Barre, L. M. (1999). Superalliance of bottlenose dolphins. Nature, 397, 571-572.

Connor, R. C., Smolker, R. A., \& Richards, A. F. (1992). Two levels of alliance formation among male bottlenose dolphins (Tursiops sp.). Proceedings of the National Academy of Sciences of the United States of America, 89, 987-990.

Conradt, L., \& Roper, T. J. (2000). Activity synchrony and social cohesion: a fission fusion model. Proceedings of the Royal Society of London B: Biological Sciences, 267, 2213-2218.

Conradt, L., \& Roper, T. J. (2005). Consensus decision making in animals. Trends in Ecology and Evolution, 20, 449-456.

Cote, J., \& Clobert, J. (2007). Social personalities influence natal dispersal in a lizard. Proceedings of the Royal Society of London B: Biological Sciences, 274, 383-390.

Cote, J., Fogarty, S., \& Sih, A. (2012). Individual sociability and choosiness between shoal types. Animal Behaviour, 83, 1469-1476.

Cote, J., Fogarty, S., Weinersmith, K., Brodin, T., \& Sih, A. (2010). Personality traits and dispersal tendency in the invasive mosquitofish (Gambusia affinis). Proceedings of the Royal Society of London B: Biological Sciences, 277, 1571-1579.

Coussi-Korbel, S., \& Fragaszy, D. M. (1995). On the relation between social dynamics and social learning. Animal Behaviour, 50, 1441-1453.

Couzin, I. D., Krause, J., Franks, N. R., \& Levin, S. A. (2005). Effective leadership and decision-making in animal groups on the move. Nature, 433, 513-516.

Couzin, I. D., Krause, J., James, R., Ruxton, G. D., \& Franks, N. R. (2002). Collective memory and spatial sorting in animal groups. Journal of Theoretical Biology, 218, $1-11$.

Croft, D. P., Arrowsmith, B. J., Bielby, J., Skinner, K., White, E., Couzin, I. D., et al. (2003). Mechanisms underlying shoal composition in the Trinidadian guppy, Poecilia reticulata. Oikos, 100, 429-438.

Croft, D. P., Edenbrow, M., Darden, S. K., Ramnarine, I. W., van Oosterhout, C., \& Cable, J. (2011). Effect of gyrodactylid ectoparasites on host behaviour and social network structure in guppies Poecilia reticulata. Behavioral Ecology and Sociobiology, 65, 2219-2227. 
Croft, D. P., James, R., \& Krause, J. (2008). Exploring animal social networks. Princeton, NJ: Princeton University Press.

Croft, D. P., James, R., Thomas, P. O. R., Hathaway, C., Mawdsley, D., Laland, K. N., et al. (2006). Social structure and co-operative interactions in a wild population of guppies (Poecilia reticulata). Behavioral Ecology and Sociobiology, 59, 644-650.

Croft, D. P., Krause, J., Darden, S. K., Ramnarine, I. W., Faria, J. J., \& James, R. (2009). Behavioural trait assortment in a social network: patterns and implications. Behavioral Ecology and Sociobiology, 63, 1495-1503.

Croft, D. P., Krause, J., \& James, R. (2004). Social networks in the guppy (Poecilia reticulata). Proceedings of the Royal Society of London B: Biological Sciences, 271, S516-S519.

Crook, J. H. (1970). Introduction-Social behaviour and ethology. In: J. H. Crook (Ed.), Social behaviour in birds and mammals: essays on the social ethology of animals and man (pp. xxi-xl). London, UK: Academic Press.

Crook, J. H., \& Gartlan, J. S. (1966). Evolution of primate societies. Nature, 210, 12001203.

Cross, P. C., Lloyd-Smith, J. O., Bowers, J. A., Hay, C. T., Hofmeyr, M., \& Getz, W. M. (2004). Integrating association data and disease dynamics in a social ungulate: bovine tuberculosis in African buffalo in the Kruger National Park. Annales Zoologici Fennici, 41, 879-892.

Danchin, É., Giraldeau, L. A., Valone, T. J., \& Wagner, R. H. (2004). Public information: from nosy neighbors to cultural evolution. Science, 305, 487-491.

Davies, N. B., Krebs, J. R., \& West, S. A. (2012). An introduction to behavioural ecology ( $4^{\text {th }}$ ed.). Hoboken, NJ: Wiley-Blackwell.

Davison, A. C., \& Hinkley, D. V. (1997). Bootstrap methods and their applications. Cambridge, UK: Cambridge University Press.

Day, R. L., MacDonald, T., Brown, C., Laland, K. N., \& Reader, S. M. (2001). Interactions between shoal size and conformity in guppy social foraging. Animal Behaviour, 62, 917-925.

Dey, C. J., Reddon, A. R., O’Connor, C. M., \& Balshine, S. (2013). Network structure is related to social conflict in a cooperatively breeding fish. Animal Behaviour, 85, 395-402. 
Dingemanse, N. J., Both, C., Drent, P. J., van Oers, K., \& van Noordwijk, A. J. (2002). Repeatability and heritability of exploratory behaviour in great tits from the wild. Animal Behaviour, 64, 929-938.

Dingemanse, N. J., van der Plas, F., Wright, J., Réale, D., Schrama, M., Roff, D. A., et al. (2009). Individual experience and evolutionary history of predation affect expression of heritable variation in fish personality and morphology. Proceedings of the Royal Society of London B: Biological Sciences, 276, 1285-1293.

Drewe, J. A., \& Perkins, S. E. (2015). Disease transmission in animal social networks. In: J. Krause, R. James, D. W. Franks, \& D. P. Croft (Eds.), Animal social networks (pp. 95-110). Oxford, UK: Oxford University Press.

Dugatkin, L. A. (1991). Dynamics of TIT FOR TAT strategy during predator inspection in the guppy (Poecilia reticulata). Behavioral Ecology and Sociobiology, 29, 127132.

Dugatkin, L. A. (1992). Sexual selection and imitation: females copy the mate choice of others. The American Naturalist, 139, 1384-1389.

Dugatkin, L. A. (2006). The altruism equation: seven scientists search for the origins of goodness. Princeton, NJ: Princeton University Press.

Dugatkin, L. A. (2013). Principles of animal behavior ( $3^{\text {rd }}$ ed.). New York, NY: W. W. Norton \& Company.

Dugatkin, L. A., \& Alfieri, M. S. (2003). Boldness, behavioral inhibition, and learning. Ethology Ecology and Evolution, 15, 43-49.

Dugatkin, L. A., \& Godin, J. G. J. (1993). Female mate copying in the guppy (Poecilia reticulata): age-dependent effects. Behavioral Ecology, 4, 289-292.

Dyer, J. R. G., Croft, D. P., Morrell, L. J., \& Krause, J. (2009). Shoal composition determines foraging success in the guppy. Behavioral Ecology, 20, 165-171.

Ekman, J. (1979). Coherence, composition, and territories of winter social groups of the willow tit Parus montanus and the crested tit P. cristatus. Ornis Scandinavica, 10, 56-68.

Elvidge, C. K., Chuard, P. J. C., \& Brown, G. E. (2016). Local predation risk shapes spatial and foraging neophobia patterns in Trinidadian guppies. Current Zoology, $62,457-462$.

Espinas, A. (1878). Des sociétés animales: étude de psychologie comparée. Paris, FR: Libraire Germer Ballière. 
Fairbanks, L. A. (1980). Relationships among adult females in captive vervet monkeys: testing a model of rank-related attractiveness. Animal Behaviour, 28, 853-859.

Farine, D. R. (2014). Measuring phenotypic assortment in animal social networks: weighted associations are more robust than binary edges. Animal Behaviour, 89, 141-153.

Farine, D. R., Aplin, L. M., Sheldon, B. C., \& Hoppitt, W. (2015). Interspecific social networks promote information transmission in wild songbirds. Proceedings of the Royal Society of London B: Biological Sciences, 282, 20142804.

Farine, D. R., Montiglio, P. O., \& Spiegel, O. (2015). From individuals to groups and back: the evolutionary implications of group phenotypic composition. Trends in Ecology and Evolution, 30, 609-621.

Farine, D. R., Strandburg-Peshkin, A., Brugere, I., Li, V. J., Berger-Wolf, T. Y., \& Crofoot, M. C. (August 2014). Self-organisation in a dynamic network of wild Anubis baboons. In: Presentation at the 15th conference of the International Society for Behavioral Ecology. New York, NY.

Faust, K. (2006). Comparing social networks: size, density, and local structure. Metodološki zvezki, 3, 185-216.

Faust, K. (2007). Very local structure in social networks. Sociological Methodology, 37, 209-256.

Faust, K. (2010). A puzzle concerning triads in social networks: graph constraints and the triad census. Social Networks, 32, 221-233.

Faust, K., \& Skvoretz, J. (2002). Comparing networks across space and time, size and species. Sociological Methodology, 32, 267-299.

Ferrari, M. C. O., Messier, F., \& Chivers, D. P. (2007). First documentation of cultural transmission of predator recognition by larval amphibians. Ethology, 113, 621627.

Fisher, J., \& Hinde, R. A. (1949). The opening of milk bottles by birds. British Birds, 42, 347-357.

Flack, A., Pettit, B., Freeman, R., Guilford, T., \& Biro, D. (2012). What are leaders made of? The role of individual experience in determining leader-follower relations in homing pigeons. Animal Behaviour, 83, 703-709.

Flack, J. C., Girvan, M., de Waal, F. B. M., \& Krakauer, D. C. (2006). Policing stabilizes construction of social niches in primates. Nature, 439, 426-429. 
Flack, J. C., Krakauer, D. C., \& de Waal, F. B. M. (2005). Robustness mechanisms in primate societies: a perturbation study. Proceedings of the Royal Society of London B: Biological Sciences, 272, 1091-1099.

Foote, J. R., Fitzsimmons, L. P., Mennill, D. J., \& Ratcliffe, L. M. (2010). Black-capped chickadee dawn choruses are interactive communication networks. Behaviour, 147, 1219-1248.

Foster, K. R., \& Ratnieks, F. L. W. (2001). Convergent evolution of worker policing by egg eating in the honeybee and common wasp. Proceedings of the Royal Society of London B: Biological Sciences, 268, 169-174.

Fournier, D. A., Skaug, H. J., Ancheta, J., Ianelli, J., Magnusson, A., Maunder, M., et al. (2012). AD Model Builder: using automatic differentiation for statistical inference of highly parameterized complex nonlinear models. Optimization Methods and Software, 27, 233-249.

Fowler, J. H., Dawes, C. T., \& Christakis, N. A. (2009). Models of genetic variation in human social networks. Proceedings of the National Academy of Sciences of the United States of America, 106, 1720-1724.

Franz, M., \& Nunn, C. L. (2009). Network-based diffusion analysis: a new method for detecting social learning. Proceedings of the Royal Society of London B:

Biological Sciences, 276, 1829-1836.

Franz, M., \& Nunn, C. L. (2010). Investigating the impact of observation errors on the statistical performance of network-based diffusion analysis. Learning and Behavior, 38, 235-242.

Freeman, L. C. (1979). Centrality in social networks: conceptual clarification. Social Networks, 1, 215-239.

Freeman, L. C. (2004). The development of social network analysis: a study in the sociology of science. Vancouver, BC: Empirical Press.

Freeman, R., Mann, R., Guilford, T., \& Biro, D. (2011). Group decisions and individual differences: route fidelity predicts flight leadership in homing pigeons (Columba livia). Biology Letters, 7, 63-66.

Galef, B. G. (2009). Strategies for social learning: testing predictions from formal theory. Advances in the Study of Behavior, 39, 117-151.

Galef Jr., B. G., Kennett, D. J., \& Wigmore, S. W. (1984). Transfer of information concerning distant foods in rats: a robust phenomenon. Animal Learning and Behavior, 12, 292-296. 
Galef, B. G., \& Whiskin, E. E. (2008). Effectiveness of familiar kin and unfamiliar nonkin demonstrator rats in altering food choices of their observers. Animal Behaviour, 76, 1381-1388.

Gaskin, D. E. (1982). The ecology of whales and dolphins. London, UK: Heinemann.

Gilby, I. C., Brent, L. J. N., Wroblewski, E. E., Rudicell, R. S., Hahn, B. H., Goodall, J., et al. (2013). Fitness benefits of coalitionary aggression in male chimpanzees. Behavioral Ecology and Sociobiology, 67, 373-381.

Godfrey, S. S., Bull, C. M., James, R., \& Murray, K. (2009). Network structure and parasite transmission in a group living lizard, the gidgee skink, Egernia stokesii. Behavioral Ecology and Sociobiology, 63, 1045-1056.

Granovetter, M. S. (1973). The strength of weak ties. American Journal of Sociology, 78, 1360-1380.

Granroth-Wilding, H. M. V., \& Magurran, A. E. (2013). Asymmetry in pay-off predicts how familiar individuals respond to one another. Biology Letters, 9, 20130025.

Greenberg, R. (2003). The role of neophobia and neophilia in the development of innovative behaviour of birds. In: S. M. Reader, \& K. N. Laland (Eds.), Animal innovation (pp. 175-196). Oxford, UK: Oxford University Press.

Griffiths, S. W., Brockmark, S., Höjesjö, J., \& Johnsson, J. I. (2004). Coping with divided attention: the advantage of familiarity. Proceedings of the Royal Society of London B: Biological Sciences, 271, 695-699.

Griffiths, S. W., \& Magurran, A. E. (1997). Familiarity in schooling fish: how long does it take to acquire? Animal Behaviour, 53, 945-949.

Griffiths, S. W., \& Magurran, A. E. (1998). Sex and schooling behaviour in the Trinidadian guppy. Animal Behaviour, 56, 689-693.

Griffiths, S. W., \& Ward, A. J. W. (2011). Social recognition of conspecifics. In: C. Brown, K. Laland, \& J. Krause (Eds.), Fish cognition and behavior $\left(2^{\text {nd }}\right.$ ed.) (pp. 186-216). Ames, IA: Wiley-Blackwell.

Gross, T., \& Blasius, B. (2008). Adaptive coevolutionary networks: a review. Journal of the Royal Society Interface, 5, 259-271.

Haccou, P., \& Meelis, E. (1994). Statistical analysis of behavioral data. New York, NY: Oxford University Press.

Hanby, J. P. (1980a). Relationships in six groups of rhesus monkeys I: networks. American Journal of Physical Anthropology, 52, 549-564. 
Hanby, J. P. (1980b). Relationships in six groups of rhesus monkeys II: dyads. American Journal of Physical Anthropology, 52, 565-575.

Hanneman, R. A., \& Riddle, M. (2005). Introduction to social network methods. Riverside, CA: University of California, Riverside.

Harary, F. (1961). Who eats whom? General Systems, 6, 41-44.

Harcourt, J. L., Ang, T. Z., Sweetman, G., Johnstone, R. A., \& Manica, A. (2009). Social feedback and the emergence of leaders and followers. Current Biology, 19, 248252.

Harris, S., Ramnarine, I. W., Smith, H. G., \& Pettersson, L.B. (2010). Picking personalities apart: estimating the influence of predation, sex and body size on boldness in the guppy Poecilia reticulata. Oikos, 119, 1711-1718.

Harvey, P. H., \& Pagel, M. D. (1991). The comparative method in evolutionary biology. Oxford, UK: Oxford University Press.

Hasenjager, M. J., \& Dugatkin, L. A. (2015). Social network analysis in behavioral ecology. Advances in the Study of Behavior, 47, 39-114.

Hasenjager, M. J., \& Dugatkin, L. A. (2017). Familiarity affects network structure and information flow in guppy (Poecilia reticulata) shoals. Behavioral Ecology, 28, 233-242.

Heathcote, R. J. P., Darden, S. K., Franks, D. W., Ramnarine, I. W., \& Croft, D. P. (2017). Fear of predation drives stable and differentiated social relationships in guppies. Scientific Reports, 7, 41679.

Hemelrijk, C. K., \& Hildenbrandt, H. (2008). Self-organized shape and frontal density of fish schools. Ethology, 114, 245-254.

Hemelrijk, C. K., \& Kunz, H. (2005). Density distribution and size sorting in fish schools: an individual-based model. Behavioral Ecology, 16, 178-187.

Herborn, K. A., Heidinger, B. J., Alexander, L., \& Arnold, K. E. (2014). Personality predicts behavioral flexibility in a fluctuating, natural environment. Behavioral Ecology, 25, 1374-1379.

Hinde, R. A. (1959). Unitary drives. Animal Behaviour, 7, 130-141.

Hinde, R. A. (1976). Interactions, relationships and social structure. Man, 11, 1-17.

Hinde, R. A. (1982). Ethology: its nature and relations with other sciences. New York, NY: Oxford University Press. 
Hobson, E. A., Avery, M. L., \& Wright, T. F. (2013). An analytical framework for quantifying and testing patterns of temporal dynamics in social networks. Animal Behaviour, 85, 83-96.

Holme, P., \& Newman, M. E. J. (2006). Nonequilibrium phase transition in the coevolution of networks and opinions. Physical Review E, 74, 056108.

Holme, P., Park, S. M., Kim, B. J., \& Edling, C. R. (2007). Korean university life in a network perspective: dynamics of a large affiliation network. Physica A: Statistical Mechanics and Its Applications, 373, 821-830.

Holme, P., \& Saramäki, J. (2012). Temporal networks. Physical Reports, 519, 97-125.

Homans, G. C. (1951). The human group. London, UK: Routledge and Kegan Paul.

Hoppitt, W., Boogert, N. J., \& Laland, K. N. (2010). Detecting social transmission in networks. Journal of Theoretical Biology, 263, 544-555.

Hoppitt, W., Kandler, A., Kendal, J. R., \& Laland, K. N. (2010). The effect of task structure on diffusion dynamics: implications for diffusion curve and networkbased analyses. Learning and Behavior, 38, 243-251.

Hoppitt, W., \& Laland, K. N. (2013). Social learning: an introduction to mechanisms, methods, and models. Princeton, NJ: Princeton University Press.

Huffman, M. A. (1996). Acquisition of innovative cultural behaviors in nonhuman primates: a case study of stone handling, a socially transmitted behavior in Japanese macaques. In: C. M. Heyes, \& B. G. Galef, Jr. (Eds.), Social learning in animals: the roots of culture (pp. 267-289). San Diego, CA: Academic Press.

Hui, A., \& Pinter-Wollman, N. (2014). Individual variation in exploratory behaviour improves speed and accuracy of collective nest selection by Argentine ants. Animal Behaviour, 93, 261-266.

Hunt, G. R., \& Gray, R. D. (2003). Diversification and cumulative evolution in New Caledonian crow tool manufacture. Proceedings of the Royal Society of London B: Biological Sciences, 270, 867-874.

Ilany, A., Barocas, A., Koren, L., Kam, M., \& Geffen, E. (2013). Structural balance in the social networks of a wild mammal. Animal Behaviour, 85, 1397-1405.

Ioannou, C. C., Couzin, I. D., James, R., Croft, D. P., \& Krause, J. (2011). Social organisation and information transfer in schooling fish. In: C. Brown, J. Krause, $\&$ K. N. Laland (Eds.), Fish cognition and behavior ( $2^{\text {nd }}$ ed.). (pp. 217-239). Hoboken, NJ: Wiley-Blackwell. 
Ioannou, C. C., Guttal, V., \& Couzin, I. D. (2012). Predatory fish select for coordinated collective motion in virtual prey. Science, 337, 1212-1215.

Ioannou, C. C., Singh, M., \& Couzin, I. D. (2015). Potential leaders trade off goaloriented and socially oriented behavior in mobile animal groups. The American Naturalist, 186, 284-293.

Jacobs, A., Sueur, C., Deneubourg, J. L., \& Petit, O. (2011). Social network influences decision making during collective movements in brown lemurs (Eulemur fulvus fulvus). International Journal of Primatology, 32, 721-736.

James, R., Croft, D. P., \& Krause, J. (2009). Potential banana skins in animal social network analysis. Behavioral Ecology and Sociobiology, 63, 989-997.

Jarman, P. J. (1974). The social organization of antelope in relation to their ecology. Behaviour, 48, 215-267.

Jeanson, R. (2012). Long-term dynamics in proximity networks in ants. Animal Behaviour, 83, 915-923.

Johannesen, A., Dunn, A. M., \& Morrell, L. J. (2012). Olfactory cue use by three-spined sticklebacks foraging in turbid water: prey detection or prey location? Animal Behaviour, 84, 151-158.

Jolles, J. W., Fleetwood-Wilson, A., Nakayama, S., Stumpe, M. C., Johnstone, R. A., \& Manica, A. (2015). The role of social attraction and its link with boldness in the collective movements of three-spined sticklebacks. Animal Behaviour, 99, 147153.

Jones, C. B. (2005). Behavioral flexibility in primates: causes and consequences. New York, NY: Springer.

Kaplan, J. R., \& Zucker, E. (1980). Social organization in a group of free-ranging patas monkeys. Folia Primatologica, 34, 196-213.

Kappeler, P. M. (1993). Variation in social structure: the effects of sex and kinship on social interactions in three lemur species. Ethology, 93, 125-145.

Kawai, M. (1965). Newly-acquired pre-cultural behavior of the natural troop of Japanese monkeys on Koshima islet. Primates, 6, 1-30.

Keiser, C. N., Pinter-Wollman, N., Augustine, D. A., Ziemba, M. J., Hao, L., Lawrence, J. G., et al. (2016). Individual differences in boldness influence patterns of social interactions and the transmission of cuticular bacteria among group-mates. Proceedings of the Royal Society of London B: Biological Sciences, 283, 20160457. 
Keller, M., Cornilleau, F. Archer, E., \& Lévy, F. (2011). Development of social familiarity in ewes. Physiology and Behavior, 104, 392-397.

Kelley, J. L., Morrell, L. J., Inskip, C., Krause, J., \& Croft, D. P. (2011). Predation risk shapes social networks in fission-fusion populations. PLoS One, 6, e24280.

Kempe, D., Kleinberg, J., \& Kumar, A. (2002). Connectivity and inference problems for temporal networks. Journal of Computer and System Sciences, 64, 820-842.

Kendal, R. L., Custance, D. M., Kendal, J. R., Vale, G., Stoinski, T. S., Rakotomalala, N. L., et al. (2010). Evidence for social learning in wild lemurs (Lemur catta). Learning and Behavior, 38, 220-234.

Kennedy, J. S. (1954). Is modern ethology objective? Animal Behaviour, 2, 12-19.

Kohn, G. M., Meredith, G. R., Magdaleno, F. R., King, A. P., \& West, M. J. (2015). Sex differences in familiarity preferences within fission-fusion brown-headed cowbird, Molothrus ater, flocks. Animal Behaviour, 106, 137-143.

Kollmann, M., Løvdok, L., Bartholomé, K., Timmer, J., \& Sourjik, V. (2005). Design principles of a bacterial signaling network. Nature, 438, 504-507.

Kostakos, V. (2009). Temporal graphs. Physica A: Statistical Mechanics and its Applications, 388, 1007-1023.

Krakauer, D. C. (1995). Groups confuse predators by exploiting perceptual bottlenecks: a connectionist model of the confusion effect. Behavioral Ecology and Sociobiology, 36, 421-429.

Krause, J., Butlin, R. K., Peuhkuri, N., \& Pritchard, V. L. (2000). The social organization of fish shoals: a test of the predictive power of laboratory experiments for the field. Biological Reviews, 75, 477-501.

Krause, J., Croft, D. P., \& James, R. (2007). Social network theory in the behavioural sciences: potential applications. Behavioral Ecology and Sociobiology, 62, 15-27.

Krause, J., James, R., \& Croft, D. P. (2010). Personality in the context of social networks. Philosophical Transactions of the Royal Society of London B: Biological Sciences, 365, 4099-4106.

Krause, J., James, R., Franks, D. W., \& Croft, D. P. (2015). Animal social networks. Oxford, UK: Oxford University Press.

Krause, J., Krause, S., Arlinghaus, R., Psorakis, I., Roberts, S., \& Rutz, C. (2013). Reality mining of animal social systems. Trends in Ecology and Evolution, 28, 541-551. 
Krause, J., Loader, S. P., McDermott, J., \& Ruxton, G. D. (1998). Refuge use by fish as a function of body length-related metabolic expenditure and predation risks.

Proceedings of the Royal Society of London B: Biological Sciences, 265, 23732379.

Krause, J., \& Ruxton, G. D. (2002). Living in groups. Oxford, UK: Oxford University Press.

Krause, S., Wilson, A. D. M., Ramnarine, I. W., Herbert-Read, J. E., Clément, R. J. G., \& Krause, J. (2016). Guppies occupy consistent positions in social networks: mechanisms and consequences. Behavioral Ecology, 28, 429-438.

Krueger, K., Flauger, B., Farmer, K., \& Hemelrijk, C. (2014). Movement initiation in groups of feral horses. Behavioural Processes, 103, 91-101.

Krützen, M., Mann, J., Heithaus, M. R., Connor, R. C., Bejder, L., \& Sherwin, W. B. (2005). Cultural transmission of tool use in bottlenose dolphins. Proceedings of the National Academy of Sciences of the United States of America, 102, 89398943.

Kuhn, F., \& Oshman, R. (2011). Dynamic networks: models and algorithms. ACM SIGACT News, 42, 82-96.

Kummer, H. (1968). Social organization of Hamadryas baboons: a field study. Chicago, IL: University of Chicago Press.

Kurvers, R. H. J. M., Krause, J., Croft, D. P., Wilson, A. D. M., \& Wolf, M. (2014). The evolutionary and ecological consequences of animal social networks: emerging issues. Trends in Ecology and Evolution, 29, 326-335.

Kurvers, R. H. J. M., Nolet, B. A., Prins, H. H. T., Ydenberg, R. C., \& van Oers, K. (2012). Boldness affects foraging decisions in barnacle geese: an experimental approach. Behavioral Ecology, 23, 1155-1161.

Kurvers, R. H. J. M., van Oers, K., Nolet, B. A., Jonker, R. M., van Wieren, S. E., Prins, H. H. T., et al. (2010). Personality predicts the use of social information. Ecology Letters, 13, 829-837.

Lachlan, R. F., Crooks, L., \& Laland, K. N. (1998). Who follows whom? Shoaling preferences and social learning of foraging information in guppies. Animal Behaviour, 56, 181-190.

Lack, D. (1968). Ecological adaptations for breeding in birds. London, UK: Chapman and Hall.

Laland, K. N. (2004). Social learning strategies. Learning and Behavior, 32, 4-14. 
Laland, K. N., \& Galef Jr., B. G. (Eds.). (2009). The question of animal culture. Cambridge, MA: Harvard University Press.

Laland, K. N., \& Janik, V. M. (2006). The animal cultures debate. Trends in Ecology and Evolution, 21, 542-547.

Laland, K. N., \& Kendal, J. R. (2003). What the models say about social learning. In: D. Fragaszy, \& S. Perry (Eds.), The biology of traditions: models and evidence (pp. 33-55). Cambridge, UK: Cambridge University Press.

Landeau, L., \& Terborgh, J. (1986). Oddity and the 'confusion effect' in predation. Animal Behaviour, 34, 1372-1380.

Laskowski, K. L., \& Bell, A. M. (2014). Strong personalities, not social niches, drive individual differences in social behaviours in sticklebacks. Animal Behaviour, 90, 287-295.

Laskowski, K. L., \& Pruitt, J.N. (2014). Evidence of social niche construction: persistent and repeated social interactions generate stronger personalities in a social spider. Proceedings of the Royal Society of London B: Biological Sciences, 281, 20133166.

Le Pendu, Y., Briedermann, L., Gerard, J. F., \& Maublanc, M. L. (1995). Inter-individual associations and social structure of a mouflon population (Ovis orientalis musimon). Behavioural Processes, 34, 67-80.

Leblond, C., \& Reebs, S. G. (2006). Individual leadership and boldness in shoals of golden shiners (Notemigonus crysoleucas). Behaviour, 143, 1263-1280.

Leenders, R. T. A. J. (1995). Models for network dynamics: a Markovian framework. Journal of Mathematical Sociology, 20, 1-21.

Lefebvre, L. (1995). Culturally-transmitted feeding behaviour in primates: evidence for accelerating learning rates. Primates, 36, 227-239.

Lefebvre, D., Ménard, N., \& Pierre, J. S. (2003). Modelling the influence of demographic parameters on group structure in social species with dispersal asymmetry and group fission. Behavioral Ecology and Sociobiology, 53, 402-410.

Lehrman, D. S. (1953). A critique of Konrad Lorenz's theory of instinctive behaviour. The Quarterly Review of Biology, 28, 337-363.

Leu, S. T., Kappeler, P. M., \& Bull, C. M. (2010). Refuge sharing network predicts ectoparasite load in a lizard. Behavioral Ecology and Sociobiology, 64, 14951503. 
Levine, J. H. (1972). The sphere of influence. American Sociological Review, 37, 14-27.

Lewin, K. (1951). Field theory in social science: selected theoretical papers. New York, NY: Harper.

Li, P. P., \& Hui, P. M. (2008). Dynamics of opinion formation in hierarchical social networks: network structure and initial bias. The European Physical Journal B, 61, 371-376.

Lima, S. L. (1998). Nonlethal effects in the ecology of predator-prey interactions. Bioscience, 48, 25-34.

Lima, S. L. \& Bednekoff, P. A. (1999). Temporal variation in danger drives antipredator behavior: the predation risk allocation hypothesis. The American Naturalist, 153, 649-659.

Lima, S. L. \& Dill, L. M. (1990). Behavioral decisions made under the risk of predation: a review and prospectus. Canadian Journal of Zoology, 68, 619-640.

Lindeyer, C. M., Meaney, M. J., \& Reader, S. M. (2013). Early maternal care predicts reliance on social learning about food in adult rats. Developmental Psychobiology, $55,168-175$.

Lorenz, K. Z. (1937). The companion in the bird's world. The Auk, 54, 245-273.

Lusseau, D. (2003). The emergent properties of a dolphin social network. Proceedings of the Royal Society of London B: Biological Sciences, 270, S186-S188.

Lusseau, D. (2007). Evidence for social role in a dolphin social network. Evolutionary Ecology, 21, 357-366.

Lusseau, D., \& Newman, M. E. J. (2004). Identifying the role that animals play in their social networks. Proceedings of the Royal Society of London B: Biological Sciences, 271, S477-S481.

Lusseau, D., Whitehead, H., \& Gero, S. (2008). Incorporating uncertainty into the study of animal social networks. Animal Behaviour, 75, 1809-1815.

MacCarthy, T., Seymour, R., \& Pomiankowski, A. (2003). The evolutionary potential of the Drosophila sex determination gene network. Journal of Theoretical Biology, 225, 461-468.

Mackinson, S. (1999). Variation in structure and distribution of pre-spawning Pacific herring shoals in two regions of British Columbia. Journal of Fish Biology, 55, 972-989. 
Magurran, A. E. (2005). Evolutionary ecology: the Trinidadian guppy. Oxford, UK: Oxford University Press.

Magurran, A. E., \& Higham, A. (1988). Information transfer across fish shoals under predator threat. Ethology, 78, 153-158.

Makagon, M. M., McCowan, B., \& Mench, J. A. (2012). How can social network analysis contribute to social behavior research in applied ethology? Applied Animal Behaviour Science, 138, 152-161.

Marchetti, C., \& Drent, P. J. (2000). Individual differences in the use of social information in foraging by captive great tits. Animal Behaviour, 60, 131-140.

Maryanski, A. R. (1987). African ape social structure: is there strength in weak ties? Social Networks, 9, 191-215.

Maynard Smith, J. (1982). Evolution and the theory of games. Cambridge, UK: Cambridge University Press.

McBride, G. (1964). A general theory of social organization and behaviour. University of Queensland Papers, Faculty of Veterinary Science, 1, 75-110.

McDonald, D. B. (2007). Predicting fate from early connectivity in a social network. Proceedings of the National Academy of Sciences of the United States of America, 104, 10910-10914.

McDonald, D. B. (2009). Young-boy networks without kin clusters in a lek-mating manakin. Behavioral Ecology and Sociobiology, 63, 1029-1034.

McDonald, D. B., \& Shizuka, D. (2013). Comparative transitive and temporal orderliness in dominance networks. Behavioral Ecology, 24, 511-520.

McDonald, N. D., Rands, S. A., Hill, F., Elder, C., \& Ioannou, C. C. (2016). Consensus and experience trump leadership, suppressing individual personality during social foraging. Science Advances, 2, e1600892.

McNamara, J. M., \& Leimar, O. (2010). Variation and the response to variation as a basis for successful cooperation. Philosophical Transactions of the Royal Society of London B: Biological Sciences, 365, 2627-2633.

McNamara, J. M., Stephens, P. A., Dall, S. R. X., \& Houston, A. I. (2009). Evolution of trust and trustworthiness: social awareness favours personality differences. Proceedings of the Royal Society of London B: Biological Sciences, 276, 605-613. 
Metcalfe, N. B., \& Thomson, B. C. (1995). Fish recognize and prefer to shoal with poor competitors. Proceedings of the Royal Society of London B: Biological Sciences, 259, 207-210.

Meyers, L. A., Pourbohloul, B., Newman, M. E. J., Skowronski, D. M., \& Brunham, R. C. (2005). Network theory and SARS: predicting outbreak diversity. Journal of Theoretical Biology, 232, 71-81.

Michelena, P., Jeanson, R., Deneubourg, J. L., \& Sibbald, A. M. (2010). Personality and collective decision-making in foraging herbivores. Proceedings of the Royal Society of London B: Biological Sciences, 277, 1093-1099.

Michelena, P., Sibbald, A. M., Erhard, H. W., \& McLeod, J. E. (2009). Effects of group size and personality on social foraging: the distribution of sheep across patches. Behavioral Ecology, 20, 145-152.

Micheletta, J., Waller, B. M., Panggur, M. R., Neumann, C., Duboscq, J., Agil, M., et al. (2012). Social bonds affect anti-predator behaviour in a tolerant species of macaque, Macaca nigra. Proceedings of the Royal Society of London B: Biological Sciences, 279, 4042-4050.

Miller, J. L., King, A. P., \& West, M. J. (2008). Female social networks influence male vocal development in brown-headed cowbirds, Molothrus ater. Animal Behaviour, 76, 931-941.

Milo, R., Itzkovitz, S., Kashtan, N., Levitt, R., Shen-Orr, S., Ayzenshtat, I., et al. (2004). Superfamilies of evolved and designed networks. Science, 303, 1538-1542.

Milo, R., Shen-Orr, S., Itzkovitz, S., Kashtan, N., Chklovskii, D., \& Alon, U. (2002). Network motifs: simple building blocks of complex networks. Science, 298, 824827.

Mineka, S., \& Cook, M. (1988). Social learning and the acquisition of snake fear in monkeys. In: B. G. Galef, Jr., \& T. R. Zentall (Eds.), Social learning: psychological and biological perspectives (pp. 51-73). Hillsdale, NJ: Lawrence Erlbaum.

Mitani, M. (1986). Voiceprint identification and its application to sociological studies of wild Japanese monkeys (Macaca fuscata yakui). Primates, 27, 397-412.

Modlmeier, A. P., Keiser, C. N., Watters, J. V., Sih, A., \& Pruitt, J. N. (2014). The keystone individual concept: an ecological and evolutionary overview. Animal Behaviour, 89, 53-62. 
Modlmeier, A. P., Laskowski, K. L., DeMarco, A. E., Coleman, A., Zhao, K., Brittingham, H. A., et al. (2014). Persistent social interactions beget more pronounced personalities in a desert-dwelling social spider. Biology Letters, 10, 20140419.

Modlmeier, A. P., Liebmann, J. E., \& Foitzik, S. (2012). Diverse societies are more productive: a lesson from ants. Proceedings of the Royal Society of London B: Biological Sciences, 279, 2142-2150.

Montiglio, P. O., Ferrari, C., \& Réale, D. (2013). Social niche specialization under constraints: personality, social interactions and environmental heterogeneity. Philosophical Transactions of the Royal Society of London B: Biological Sciences, 368, 20120343.

Moreno, J. L. (1934). Who shall survive? A new approach to the problem of human interrelations. Washington, DC: Nervous and Mental Disease Publishing Co.

Morgan, B. J. T. (2009). Applied stochastic modeling (2 ${ }^{\text {nd }}$ ed.). Boca Raton, FL: Chapman \& Hall/CRC Press.

Morrell, L. J., Croft, D. P., Dyer, J. R. G., Chapman, B. B., Kelley, J. L., Laland, K. N., et al. (2008). Association patterns and foraging behaviour in natural and artificial guppy shoals. Animal Behaviour, 76, 855-864.

Morrell, L. J., Hunt, K. L., Croft, D. P., \& Krause, J. (2007). Diet, familiarity and shoaling decisions in guppies. Animal Behaviour, 74, 311-319.

Mourier, J., Vercelloni, J., \& Planes, S. (2012). Evidence of social communities in a spatially structured network of a free-ranging shark species. Animal Behaviour, $83,389-401$.

Myers, J. P. (1983). Space, time and the pattern of individual associations in a groupliving species: sanderlings have no friends. Behavioral Ecology and Sociobiology, $12,129-134$.

Nagy, M., Vásárhelyi, G., Pettit, B., Roberts-Mariani, I., Vicsek, T., \& Biro, D. (2013). Context-dependent hierarchies in pigeons. Proceedings of the National Academy of Sciences of the United States of America, 110, 13049-13054.

Nakagawa, N. (1992). Distribution of affiliative behaviors among adult females within a group of wild patas monkeys in a nonmating, nonbirth season. International Journal of Primatology, 13, 73-96.

Nakagawa, S., \& Schielzeth, H. (2010). Repeatability for Gaussian and non-Gaussian data: a practical guide for biologists. Biological Reviews, 85, 935-956. 
Nakayama, S., Harcourt, J. L., Johnstone, R. A., \& Manica, A. (2016). Who directs group movement? Leader effort versus follower preference in stickleback fish of different personality. Biology Letters, 12, 20160207.

Nardini, C., Kozma, B., \& Barrat, A. (2008). Who's talking first? Consensus or lack thereof in coevolving opinion formation models. Physical Review Letters, 100, 158701 .

Naug, D. (2008). Structure of the social network and its influence on transmission dynamics in a honeybee colony. Behavioral Ecology and Sociobiology, 62, 17191725.

Newman, M. E. J. (2002). Assortative mixing in networks. Physical Review Letters, 89, 208701 .

Newman, M. E. J. (2003). The structure and function of complex networks. SIAM Review, 45, 167-256.

Newman, M. E. J. (2004). Analysis of weighted networks. Physical Review E, 70, 056131.

Newman, M. E. J. (2006). Modularity and community structure in networks. Proceedings of the National Academy of Sciences of the United States of America, 103, 85778582 .

Noad, M. J., Cato, D. H., Bryden, M. M., Jenner, M. N., \& Jenner, K. C. S. (2000). Cultural revolution in whale songs. Nature, 408, 537.

Nonacs, P., \& Kapheim, K. M. (2007). Social heterosis and the maintenance of genetic diversity. Journal of Evolutionary Biology, 20, 2253-2265.

Nowak, M. A., \& May, R. M. (1992). Evolutionary games and spatial chaos. Nature, 359, 826-829.

Nowak, M. A., \& Sigmund, K. (1992). Tit for tat in heterogeneous populations. Nature, $355,250-253$.

Nowak, M. A., Tarnita, C. E., \& Antal, T. (2010). Evolutionary dynamics in structured populations. Philosophical Transactions of the Royal Society of London B: Biological Sciences, 365, 19-30.

Oh, K. P., \& Badyaev, A. V. (2010). Structure of social networks in a passerine bird: consequences for sexual selection and the evolution of mating strategies. The American Naturalist, 176, E80-E89. 
Ohtsuki, H., Hauert, C., Lieberman, E., \& Nowak, M. A. (2006). A simple rule for the evolution of cooperation on graphs and social networks. Nature, 441, 502-505.

Pearl, M. C., \& Schulman, S. R. (1983). Techniques for the analysis of social structure in animal societies. Advances in the Study of Behavior, 13, 107-146.

Petit, O., \& Bon, R. (2010). Decision-making processes: the case of collective movements. Behavioral Processes, 84, 635-647.

Petrucci, R. (1905). Les origines naturelles de la propriété: Essai de sociologie comparée. Instituts Solvay. Travaux de l'Institut de Sociologie. Notes et Memoires. Fasc. 3. Bruxelles, BE: Misch et Thron.

Petrucci, R. (1906). Origine polyphylétique, homotypie et non-comparabilité directe des sociétés animales. Instituts Solvay. Travaux de l'Institut de Sociologie. Notes et Memoires. Fasc. 7. Bruxelles, BE: Misch et Thron.

Pike, T. W., Samanta, M., Lindström, J., \& Royle, N. J. (2008). Behavioural phenotype affects social interactions in an animal network. Proceedings of the Royal Society of London B: Biological Sciences, 275, 2515-2520.

Pinter-Wollman, N., Hobson, E. A., Smith, J. E., Edelman, A. J., Shizuka, D., de Silva, S., et al. (2014). The dynamics of animal social networks: analytical, conceptual, and theoretical advances. Behavioral Ecology, 25, 242-255.

Pinter-Wollman, N., Isbell, L. A., \& Hart, L. A. (2009). The relationship between social behaviour and habitat familiarity in African elephants (Loxodonta africana). Proceedings of the Royal Society of London B: Biological Sciences, 276, 10091014.

Pinter-Wollman, N., Wollman, R., Guetz, A., Holmes, S., \& Gordon, D. M. (2011). The effect of individual variation on the structure and function of interaction networks in harvester ants. Journal of the Royal Society Interface, 8, 1562-1573.

Pruitt, J. N., \& Goodnight, C. J. (2014). Site-specific group selection drives locally adapted group compositions. Nature, 514, 359-362.

Pruitt, J. N., \& Riechert, S. E. (2011). How within-group behavioural variation and task efficiency enhance fitness in a social group. Proceedings of the Royal Society of London B: Biological Sciences, 278, 1209-1215.

Pulliam, H. R. (1973). On the advantages of flocking. Journal of Theoretical Biology, 38, 419-422.

Qiu, F., \& Hu, X. (2010). Modeling group structures in pedestrian crowd simulation. Simulation Modelling Practice and Theory, 18, 190-205. 
R Core Team. (2016). R: a language and environment for statistical computing. R Foundation for Statistical Computing, Vienna, Austria. Available from: https://www.R-project.org/ (accessed 9 March, 2017).

Ramseyer, A., Boissy, A., Thierry, B., \& Dumont, B. (2009). Individual and social determinants of spontaneous group movements in cattle and sheep. Animal, 3, 1319-1326.

Ratnieks, F. L. W., \& Wenseleers, T. (2005). Policing insect societies. Science, 307, 5456.

Rausher, M. D., Miller, R. E., \& Tiffin, P. (1999). Patterns of evolutionary rate variation among genes of the anthocyanin biosynthetic pathway. Molecular Biology and Evolution, 16, 266-274.

Reader, S. M. (2000). Social learning and innovation: individual differences, diffusion dynamics and evolutionary issues (Ph.D. thesis). Cambridge, UK: University of Cambridge.

Reader, S. M. (2004). Distinguishing social and asocial learning using diffusion dynamics. Learning and Behavior, 32, 90-104.

Reader, S. M., \& Laland, K. N. (2000). Diffusion of foraging innovations in the guppy. Animal Behaviour, 60, 175-180.

Réale, D., Reader, S. M., Sol, D., McDougall, P. T., \& Dingemanse, N. J. (2007). Integrating animal temperament within ecology and evolution. Biological Reviews, 82, 291-318.

Reebs, S. G. (2000). Can a minority of informed leaders determine the foraging movements of a fish shoal? Animal Behaviour, 59, 403-409.

Rhine, R. J. (1973). Variation and consistency in the social behavior of two groups of stumptail macaques (Macaca arctoides). Primates, 14, 21-35.

Ripple, W. J., \& Beschta, R. L. (2004). Wolves and the ecology of fear: can predation risk structure ecosystems? BioScience, 54, 755-766.

Rodgers, G. M., Downing, B., \& Morrell, L. J. (2015). Prey body size mediates the predation risk associated with being "odd". Behavioral Ecology, 26, 242-246.

Rogers, A. R. (1988). Does biology constrain culture? American Anthropologist, 90, 819831. 
Roney, J. R., \& Maestripieri, D. (2003). Social development and affiliation. In: D. Maestripieri (Ed.), Primate psychology (pp. 171-204). Cambridge, MA: Harvard University Press.

Rosenthal, S. B., Twomey, C. R., Hartnett, A. T., Wu, H. S., \& Couzin, I. D. (2015). Revealing the hidden networks of interaction in mobile animal groups allows prediction of complex behavioral contagion. Proceedings of the National Academy of Sciences of the United States of America, 112, 4690-4695.

Royle, N. J., Pike, T. W., Heeb, P., Richner, H., \& Kölliker, M. (2012). Offspring social network structure predicts fitness in families. Proceedings of the Royal Society of London B: Biological Sciences, 279, 4914-4922.

Ryder, T. B., McDonald, D. B., Blake, J. G., Parker, P. G., \& Loiselle, B. A. (2008). Social networks in the lek-mating wire-tailed manakin (Pipra filicauda). Proceedings of the Royal Society of London B: Biological Sciences, 275, 13671374.

Sade, D. S. (1965). Some aspects of parent-offspring and sibling relations in a group of rhesus monkeys, with a discussion of grooming. American Journal of Physical Anthropology, 23, 1-17.

Sade, D. S. (1972). Sociometrics of Macaca mulatta I. Linkages and cliques in grooming matrices. Folia Primatologica, 18, 196-223.

Sade, D. S., Altmann, M., Loy, J., Hausfater, G., \& Breuggeman, J. A. (1988).

Sociometrics of Macaca mulatta: II. Decoupling centrality and dominance in rhesus monkey social networks. American Journal of Physical Anthropology, 77, 409-425.

Sade, D. S., \& Dow, M. M. (1994). Primate social networks. In: S. Wasserman, \& J. Galaskiewicz (Eds.), Advances in social network analysis: research in the social and behavioral sciences (pp. 152-166). Thousand Oaks, CA: Sage Publications.

Saltz, J. B., Geiger, A. P., Anderson, R., Johnson, B., \& Marren, R. (2016). What, if anything, is a social niche? Evolutionary Ecology, 30, 349-364.

Santoro, N., Quattrociocchi, W., Flocchini, P., Casteigts, A., \& Amblard, F. (2011). Time-varying graphs and social network analysis: temporal indicators and metrics. arXiv, 1102.0629.

Santos, F. C., Pacheco, J. M., \& Lenaerts, T. (2006a). Evolutionary dynamics of social dilemmas in structured heterogeneous populations. Proceedings of the National Academy of Sciences of the United States of America, 103, 3490-3494. 
Santos, F. C., Pacheco, J. M., \& Lenaerts, T. (2006b). Cooperation prevails when individuals adjust their social ties. PLoS Computational Biology, 2, 1284-1291.

Schnoell, A. V., \& Fichtel, C. (2012). Wild redfronted lemurs (Eulemur rufifrons) use social information to learn new foraging techniques. Animal Cognition, 15, 505516.

Schürch, R., Rothenberger, S., \& Heg, D. (2010). The building-up of social relationships: behavioural types, social networks and cooperative breeding in a cichlid. Philosophical Transactions of the Royal Society of London B: Biological Sciences, 365, 4089-4098.

Scott, J. (2000). Social network analysis: a handbook (2 ${ }^{\text {nd }}$ ed.). Thousand Oaks, CA: Sage Publications.

Scott, J., \& Carrington, P. J. (2011). The SAGE handbook of social network analysis. Thousand Oaks, CA: Sage Publications.

Scott, J. P. (1945). Group formation determined by social behavior: a comparative study of two mammalian societies. Sociometry, 8, 42-52.

Seppänen, J. T. \& Forsman, J. T. (2007). Interspecific social learning: novel preference can be acquired from a competing species. Current Biology, 17, 1248-1252.

Seyfarth, R. M. (1976). Social relationships among adult female baboons. Animal Behaviour, 24, 917-938.

Seyfarth, R. M. (1977). A model of social grooming among adult female monkeys. Journal of Theoretical Biology, 65, 671-698.

Seyfarth, R. M. (1980). The distribution of grooming and related behaviours among adult female vervet monkeys. Animal Behaviour, 28, 798-813.

Seyfarth, R. M., \& Cheney, D. L. (1984). Grooming, alliances and reciprocal altruism in vervet monkeys. Nature, 308, 541-543.

Shizuka, D., \& McDonald, D. B. (2012). A social network perspective on measurements of dominance hierarchies. Animal Behaviour, 83, 925-934.

Sih, A., \& Bell, A. M. (2008). Insights for behavioral ecology from behavioral syndromes. Advances in the Study of Behavior, 38, 227-281.

Sih, A., Bell, A., \& Johnson, J. C. (2004). Behavioral syndromes: an ecological and evolutionary overview. Trends in Ecology and Evolution, 19, 372-378. 
Sih, A., Bell, A., \& Johnson, J. C. (2010). Behavioral syndromes. In: D. F. Westneat, \& C. W. Fox (Eds.), Evolutionary behavioral ecology (pp. 516-530). Oxford, UK: Oxford University Press.

Sih, A., Hanser, S. F., \& McHugh, K. A. (2009). Social network theory: new insights and issues for behavioral ecologists. Behavioral Ecology and Sociobiology, 63, 975988.

Sih, A., \& Watters, J. V. (2005). The mix matters: behavioural types and group dynamics in water striders. Behaviour, 142, 1417-1431.

Silk, J. B. (2007). The adaptive value of sociality in mammalian groups. Philosophical Transactions of the Royal Society of London B: Biological Sciences, 362, 539559.

Simons, A. M. (2004). Many wrongs: the advantage of group navigation. Trends in Ecology and Evolution, 19, 453-455.

Skaug, H., Fournier, D., Bolker, B., Magnusson, A., \& Nielsen, A. (2014). Generalized linear mixed models using $A D$ Model Builder. $\mathrm{R}$ package version 0.8.0.

Smith, B. R., \& Blumstein, D. T. (2008). Fitness consequences of personality: a metaanalysis. Behavioral Ecology, 19, 448-455.

Smith, B. R., \& Blumstein, D. T. (2010). Behavioral types as predictors of survival in Trinidadian guppies (Poecilia reticulata). Behavioral Ecology, 21, 919-926.

Snijders, L., van Rooij, E. P., Burt, J. M., Hinde, C. A., van Oers, K., \& Naguib, M. (2014). Social networking in territorial great tits: slow explorers have the least central social network positions. Animal Behaviour, 98, 95-102.

Snijders, T. A. B. (2001). The statistical evaluation of social network dynamics. Sociological Methodology, 31, 361-395.

Snijders, T. A. B., van de Bunt, G. G., \& Steglich, C. E. G. (2010). Introduction to stochastic actor-based models for network dynamics. Social Networks, 32, 44-60.

Soczka, L. (1974). Ethologie sociale et sociometrie: analyse de la structure d'un groupe de singes crabiers (Macaca fascicularis = irus) en captivite. Behaviour, 50, 254269.

Solé, R. V., \& Montoya, J. M. (2001). Complexity and fragility in ecological networks. Proceedings of the Royal Society of London B: Biological Sciences, 268, 20392045. 
Stamps, J., \& Groothuis, T. G. G. (2010). The development of animal personality: relevance, concepts and perspectives. Biological Reviews, 85, 301-325.

Stankowich, T., \& Blumstein, D. T. (2005). Fear in animals: a meta-analysis and review of risk assessment. Proceedings of the Royal Society of London B: Biological Sciences, 272, 2627-2634.

Stephenson, K., \& Zelen, M. (1989). Rethinking centrality: methods and examples. Social Networks, 11, 1-37.

Strandburg-Peshkin, A., Farine, D. R., Couzin, I. D., \& Crofoot, M. C. (August 2014). Leadership and collective motion in Anubis baboons using high-resolution GPS tracking. In: Presentation at the 15th conference of the International Society for Behavioral Ecology. New York, NY.

Strandburg-Peshkin, A., Twomey, C. R., Bode, N. W. F., Kao, A. B., Katz, Y., Ioannou, C. C., et al. (2013). Visual sensory networks and effective information transfer in animal groups. Current Biology, 23, R709-R711.

Strier, K. B., Boubli, J. P., Possamai, C. B., \& Mendes, S. L. (2006). Population demography of northern muriquis (Brachyteles hypoxanthus) at the Estação Biológica de Caratinga/Reserva particular do Patrimônio Natural-Felìciano Miguel Abdala, Minas Gerais, Brazil. American Journal of Physical Anthropology, 130, 227-237.

Sueur, C., Deneubourg, J. L., \& Petit, O. (2012). From social network (centralized vs. decentralized) to collective decision-making (unshared vs. shared consensus). PLoS One, 7, e32566.

Sueur, C., \& Maire, A. (2014). Modelling animal group fission using social network dynamics. PLoS One, 9, e97813.

Sueur, C., \& Petit, O. (2008). Shared or unshared consensus decision in macaques? Behavioural Processes, 78, 84-92.

Sueur, C., Petit, O., \& Deneubourg, J. L. (2009). Selective mimetism at departure in collective movements of Macaca tonkeana: an experimental and theoretical approach. Animal Behaviour, 78, 1087-1095.

Sueur, C., Petit, O., \& Deneubourg, J. L. (2010). Short-term group fission processes in macaques: a social networking approach. The Journal of Experimental Biology, 213, 1338-1346.

Sumpter, D. J. T. (2006). The principles of collective animal behaviour. Philosophical Transactions of the Royal Society of London B: Biological Sciences, 361, 5-22. 
Sundaresan, S. R., Fischhoff, I. R., Dushoff, J., \& Rubenstein, D. I. (2007). Network metrics reveal differences in social organization between two fission-fusion species, Grevy's zebra and onager. Oecologia, 151, 140-149.

Swaney, W., Kendal, J., Capon, H., Brown, C., \& Laland, K. N. (2001). Familiarity facilitates social learning of foraging behaviour in the guppy. Animal Behaviour, 62, 591-598.

Thompson, W. R. (1958). Social behaviour. In: A. Roe, \& G. G. Simpson (Eds.), Behavior and evolution (pp. 291-310). New Haven, CT: Yale University Press.

Thorpe, W. H. (1956). Learning and instinct in animals. London, UK: Methuen.

Tinbergen, N. (1953). Social behaviour in animals, with special reference to vertebrates. London, UK: Methuen.

Tokuda, M., Boubli, J. P., Mourthé, Í., Izar, P., Possamai, C. B., \& Strier, K. B. (2014). Males follow females during fissioning of a group of northern muriquis. American Journal of Primatology, 76, 529-538.

Trompf, L., \& Brown, C. (2014). Personality affects learning and trade-offs between private and social information in guppies, Poecilia reticulata. Animal Behaviour, 88, 99-106.

Underwood, R. (1981). Companion preference in an eland herd. African Journal of Ecology, 19, 341-354.

Valone, T. J. (2007). From eavesdropping on performance to copying the behavior of others: a review of public information use. Behavioral Ecology and Sociobiology, $62,1-14$.

van Oers, K., Klunder, M., \& Drent, P. J. (2005). Context-dependence of personalities: risk-taking behavior in a social and a nonsocial situation. Behavioral Ecology, 16, 716-723.

VanderWaal, K. L., Atwill, E. R., Isbell, L. A., \& McCowan, B. (2014). Linking social and pathogen transmission networks using microbial genetics in giraffe (Giraffa camelopardalis). Journal of Animal Ecology, 83, 406-414.

VanderWaal, K. L., Wang, H., McCowan, B., Fushing, H., \& Isbell, L. A. (2014). Multilevel social organization and space use in reticulated giraffe (Giraffa camelopardalis). Behavioral Ecology, 25, 17-26.

Vital, C., \& Martins, E. P. (2011). Strain differences in zebrafish (Danio rerio) social roles and their impact on group task performance. Journal of Comparative Psychology, 125, 278-285. 
Vital, C., \& Martins, E. P. (2013). Socially-central zebrafish influence group behavior more than those on the social periphery. PLoS One, 8, e55503.

Voelkl, B., Firth, J. A., \& Sheldon, B. C. (2016). Nonlethal predator effects on the turnover of wild bird flocks. Scientific Reports, 6, 33476.

Voelkl, B., \& Kasper, C. (2009). Social structure of primate interaction networks facilitates the emergence of cooperation. Biology Letters, 5, 462-464.

Voelkl, B., \& Noë, R. (2008). The influence of social structure on the propagation of social information in artificial primate groups: a graph-based simulation approach. Journal of Theoretical Biology, 252, 77-86.

Voelkl, B., \& Noë, R. (2010). Simulation of information propagation in real-life primate networks: longevity, fecundity, fidelity. Behavioral Ecology and Sociobiology, 64, 1449-1459.

de Waal, F. B. M., \& Harcourt, A. H. (1992). Coalitions and alliances: a history of ethological research. In: A. H. Harcourt, \& F. B. M. de Waal (Eds.), Coalitions and alliances in humans and other animals (pp. 1-19). Oxford, UK: Oxford University Press.

Wang, Y., \& Qian, X. (2014). Functional module identification in protein interaction networks by interaction patterns. Bioinformatics, 30, 81-93.

Ward, A. J. W., \& Webster, M. M. (2016). Sociality: the behaviour of group-living animals. Cham, CH: Springer International Publishing.

Ward, A. J. W., Webster, M. M., Magurran, A. E., Currie, S., \& Krause, J. (2009). Species and population differences in social recognition between fishes: a role for ecology? Behavioral Ecology, 20, 511-516.

Wasserman, S. S. (1977). Stochastic models for directed graphs (Ph.D. dissertation). Cambridge, MA: Harvard University.

Wasserman, S., \& Faust, K. (1994). Social network analysis: methods and applications. Cambridge, UK: Cambridge University Press.

Waters, J. S., \& Fewell, J. H. (2012). Information processing in social insect networks. PLoS One, 7, e40337.

Watts, D. J., \& Strogatz, S. H. (1998). Collective dynamics of 'small-world' networks. Nature, 393, 440-442. 
Webster, M. M., Atton, N., Hoppitt, W. J. E., \& Laland, K. N. (2013). Environmental complexity influences association network structure and network-based diffusion of foraging information in fish shoals. The American Naturalist, 181, 235-244.

Webster, M. M., Goldsmith, J., Ward, A. J. W., \& Hart, P. J. B. (2007). Habitat-specific chemical cues influence association preferences and shoal cohesion in fish.

Behavioral Ecology and Sociobiology, 62, 273-280.

Webster, M. M., \& Laland, K. N. (2008). Social learning strategies and predation risk: minnows copy only when using private information would be costly. Proceedings of the Royal Society of London B: Biological Sciences, 275, 2869-2876.

Webster, M. M., \& Ward, A. J. W. (2011). Personality and social context. Biological Reviews, 86, 759-773.

Webster, M. M., Ward, A. J. W., \& Hart, P. J. B. (2007). Boldness is influenced by social context in threespine sticklebacks (Gasterosteus aculeatus). Behaviour, 144, 351371.

Wey, T. W., \& Blumstein, D. T. (2012). Social attributes and associated performance measures in marmots: bigger male bullies and weakly affiliating females have higher annual reproductive success. Behavioral Ecology and Sociobiology, 66, 1075-1085.

Wey, T., Blumstein, D. T., Shen, W., \& Jordán, F. (2008). Social network analysis of animal behaviour: a promising tool for the study of sociality. Animal Behaviour, 75, 333-344.

Whitehead, H. (1995). Investigating structure and temporal scale in social organizations using identified individuals. Behavioral Ecology, 6, 199-208.

Whitehead, H. (1997). Analysing animal social structure. Animal Behaviour, 53, 10531067.

Whitehead, H. (2008). Analyzing animal societies: quantitative methods for vertebrate social analysis. Chicago, IL: University of Chicago Press.

Whitehead, H. (2009). SOCPROG programs: analysing animal social structures. Behavioral Ecology and Sociobiology, 63, 765-778.

Whitehead, H., \& Dufault, S. (1999). Techniques for analyzing vertebrate social structure using identified individuals: review and recommendations. Advances in the Study of Behavior, 28, 33-74.

Whitehead, H., \& Lusseau, D. (2012). Animal social networks as substrate for cultural behavioural diversity. Journal of Theoretical Biology, 294, 19-28. 
Wilson, A. D. M., Krause, S., Dingemanse, N. J., \& Krause, J. (2013). Network position: a key component in the characterization of social personality types. Behavioral Ecology and Sociobiology, 67, 163-173.

Wilson, A. D. M., Krause, S., James, R., Croft, D. P., Ramnarine, I. W., Borner, K. K., et al. (2014). Dynamic social networks in guppies (Poecilia reticulata). Behavioral Ecology and Sociobiology, 68, 915-925.

Wilson, A. D. M., Krause, S., Ramnarine, I. W., Borner, K. K., Clément, R. J. G., Kurvers, R. H. J. M., et al. (2015). Social networks in changing environments. Behavioral Ecology and Sociobiology, 69, 1617-1629.

Wilson, D. S., \& Dugatkin, L. A. (1997). Group selection and assortative interactions. The American Naturalist, 149, 336-351.

Wilson, D. S., Pollock, G. B., \& Dugatkin, L. A. (1992). Can altruism evolve in purely viscous populations? Evolutionary Ecology, 6, 331-341.

Wilson, E. O. (1975). Sociobiology: the new synthesis. Cambridge, MA: Harvard University Press.

Wiszniewski, J., Brown, C., \& Möller, L. M. (2012). Complex patterns of male alliance formation in a dolphin social network. Journal of Mammalogy, 93, 239-250.

Wolf, M., \& Krause, J. (2014). Why personality differences matter for social functioning and social structure. Trends in Ecology and Evolution, 29, 306-308.

Wolf, M., van Doorn, G. S., \& Weissing, F. J. (2011). On the coevolution of social responsiveness and behavioural consistency. Proceedings of the Royal Society of London B: Biological Sciences, 278, 440-448.

Wolf, M., \& Weissing, F. J. (2010). An explanatory framework for adaptive personality differences. Philosophical Transactions of the Royal Society of London B:

Biological Sciences, 365, 3959-3968.

Wolf, M., \& Weissing, F. J. (2012). Animal personalities: consequences for ecology and evolution. Trends in Ecology and Evolution, 27, 452-461.

Zajonc, R. B. (1965). Social facilitation. Science, 149, 269-274.

Zanette, L. Y., White, A. F., Allen, M. C., \& Clinchy, M. (2011). Perceived predation risk reduces the number of offspring songbirds produce per year. Science, 334, 1398-1401.

Žiberna, A. (2014). Blockmodeling of multilevel networks. Social Networks, 39, 46-61. 
Zuur, A. F., Ieno, E. N., Walker, N. J., Saveliev, A. A., \& Smith, G. M. (2009). Mixed effects models and extensions in ecology with R. New York, NY: Springer Science + Business Media. 


\section{APPENDIX}

\section{Copyright permissions for Chapter I}

Chapter I Text

Dear Mr. Hasenjager,

We hereby grant you permission to reprint the aforementioned material at no charge in your thesis, in print and on the University of Louisville web site subject to the following conditions:

1. If any part of the material to be used (for example, figures) has appeared in our publication with credit or acknowledgement to another source, permission must also be sought from that source. If such permission is not obtained then that material may not be included in your publication/copies.

2. Suitable acknowledgment to the source must be made, either as a footnote or in a reference list at the end of your publication, as follows:

"This article was published in Publication title, Vol number, Author(s), Title of article, Page Nos, Copyright Elsevier (or appropriate Society name) (Year).”

3. Your thesis may be submitted to your institution in either print or electronic form.

4. Reproduction of this material is confined to the purpose for which permission is hereby given.

5. This permission is granted for non-exclusive world English rights only. For other languages please reapply separately for each one required. Permission excludes use in an electronic form other than as specified above. Should you have a specific electronic project in mind please reapply for permission.

6. This includes permission for UMI to supply single copies, on demand, of the complete thesis. Should your thesis be published commercially, please reapply for permission.

Kind regards

Laura Pritchard 
Permissions Supervisor - Global Rights Department |ELSEVIER |

The Boulevard| Langford Lane | Kidlington | Oxford OX5 1GB |

Tel: +441865843517 Fax: $+\underline{441865853333}$

1.pritchard@elsevier.com

Title: Mr. Matthew Hasenjager

Institute/company: University of Louisville

Address: 1407 S 1st St, Apt 3

Post/Zip Code: 40208

City: Louisville

State/Territory: Kentucky

Country: United States

Telephone: $\underline{2482147320}$

Email: hasenjag27@gmail.com

Type of Publication: Book

Book Title: Advances in the Study of Behavior

Book ISBN: 978-0-12-802276-4

Book Author: Edited by Marc Naguib, H. Jane Brockmann, John C. Mitani, Leigh W.

Simmons, Louise Barrett, Sue Healy and Peter J.B. Slater

Book Year: 2015

Book Pages: 39 to 114

Book Chapter number: 3

Book Chapter title: Social Network Analysis in Behavioral Ecology

I would like to use: Full article/chapter

Are you the author of the Elsevier material? Yes

In what format will you use the material? Print and Electronic

Will you be translating the material? No

Information about proposed use: Reuse in a thesis/dissertation 
Figures $1.1 \& 1.2$

\section{JOHN WILEY AND SONS LICENSE TERMS AND CONDITIONS}

Apr 10, 2017

This Agreement between Matthew J Hasenjager ("You") and John Wiley and Sons ("John Wiley and Sons") consists of your license details and the terms and conditions provided by John Wiley and Sons and Copyright Clearance Center.

License Number

License date

Licensed Content Publisher

Licensed Content Publication

Licensed Content Title

Licensed Content Author

Licensed Content Date

Licensed Content Pages

Type of use

Requestor type

Format

Portion

Number of figures/tables

Original Wiley figure/table number(s)

Will you be translating?

Title of your thesis / dissertation

Expected completion date

Expected size (number of pages)

Requestor Location
4085361082930

Apr 10, 2017

John Wiley and Sons

Social network analysis in the study of nonhuman primates: A historical perspective

Lauren J.N. Brent, Julia Lehmann, Gabriel RamosFernández

Mar 23, 2011

11

Dissertation/Thesis

University/Academic

Print and electronic

Figure/table

2

Figure 1a \& Figure 2

No

Social dynamics, network structure, and information diffusion in fish shoals

Apr 2017

270

Matthew J Hasenjager

1407 South 1st Street 
Apartment 3

LOUISVILLE, KY 40208

United States

Attn: Matthew J Hasenjager

Publisher Tax ID

EU826007151

Billing Type

Invoice

Billing Address

Matthew J Hasenjager 1407 South 1st Street

Apartment 3

LOUISVILLE, KY 40208

United States

Attn: Matthew J Hasenjager

Total

0.00 USD 
Figure 1.3

\section{SPRINGER LICENSE TERMS AND CONDITIONS}

Apr 11, 2017

This Agreement between Matthew J Hasenjager ("You") and Springer ("Springer") consists of your license details and the terms and conditions provided by Springer and Copyright Clearance Center.

License Number $\quad 4085950306125$

License date

Licensed Content Springer

Publisher

Licensed Content Behavioral Ecology and Sociobiology

Publication

Licensed Content Title Simulation of information propagation in real-life primate networks: longevity, fecundity, fidelity

Licensed Content Author Bernhard Voelkl

Licensed Content Date Jan 1, 2010

Licensed Content 64

Volume

Licensed Content Issue 9

Type of Use Thesis/Dissertation

Portion Figures/tables/illustrations

Number of 1

figures/tables/illustration

$\mathrm{S}$

Author of this Springer No

article

Order reference number

Original figure numbers Figure 1

Title of your thesis / Social dynamics, network structure, and information diffusion dissertation in fish shoals

Expected completion Apr 2017 


$\begin{array}{ll}\text { date } & \\ \text { Estimated size(pages) } & 270 \\ \text { Requestor Location } & \begin{array}{l}\text { Matthew J Hasenjager } \\ \text { 1407 South 1st Street } \\ \text { Apartment 3 }\end{array} \\ & \text { LOUISVILLE, KY 40208 } \\ & \text { United States } \\ & \text { Attn: Matthew J Hasenjager } \\ & \text { Invoice } \\ & \text { Matthew J Hasenjager } \\ \text { Billing Type } & \text { Apartment 3 } \\ \text { Billing Address } & \\ & \text { LOUISVILLE, KY 40208 } \\ & \text { United States } \\ & \text { Attn: Matthew J Hasenjager } \\ & \text { 0.00 USD }\end{array}$


Figure 1.4

\section{SPRINGER LICENSE TERMS AND CONDITIONS}

Apr 11, 2017

This Agreement between Matthew J Hasenjager ("You") and Springer ("Springer") consists of your license details and the terms and conditions provided by Springer and Copyright Clearance Center.

License Number $\quad 4085950245434$

License date

Licensed Content Springer

Publisher

Licensed Content Behavioral Ecology and Sociobiology

Publication

Licensed Content Title Dynamic social networks in guppies (Poecilia reticulata)

Licensed Content Author Alexander D. M. Wilson

Licensed Content Date Jan 1, 2014

Licensed Content $\quad 68$

Volume

Licensed Content Issue 6

Type of Use Thesis/Dissertation

Portion Figures/tables/illustrations

Number of 1

figures/tables/illustration

S

Author of this Springer No

article

Order reference number

Original figure numbers Figure 1

Title of your thesis / Social dynamics, network structure, and information diffusion dissertation in fish shoals

Expected completion Apr 2017

date 


$\begin{array}{ll}\text { Estimated size(pages) } & 270 \\ \text { Requestor Location } & \text { Matthew J Hasenjager } \\ & \text { 1407 South 1st Street } \\ & \text { Apartment 3 } \\ & \text { LOUISVILLE, KY 40208 } \\ & \text { United States } \\ & \text { Attn: Matthew J Hasenjager } \\ & \text { Invoice } \\ & \text { Matthew J Hasenjager } \\ \text { Billing Type } & \text { A07 South 1st Street } \\ \text { Billing Address } & \text { Apartment 3 } \\ & \text { LOUISVILLE, KY 40208 } \\ & \text { United States } \\ & \text { Attn: Matthew J Hasenjager } \\ & \text { 0.00 USD }\end{array}$


Figure 1.5

\section{ELSEVIER LICENSE TERMS AND CONDITIONS}

Apr 10, 2017

This Agreement between Matthew J Hasenjager ("You") and Elsevier ("Elsevier") consists of your license details and the terms and conditions provided by Elsevier and Copyright Clearance Center.

License Number $\quad 4085371201227$

License date Apr 10, 2017

Licensed Content Elsevier

Publisher

Licensed Content Journal of Theoretical Biology

Publication

Licensed Content Title Collective Memory and Spatial Sorting in Animal Groups

Licensed Content IAIN D. COUZIN, JENS KRAUSE, RICHARD JAMES, Author GRAEME D. RUXTON, NIGEL R. FRANKS

Licensed Content Date 7 September 2002

Licensed Content 218

Volume

Licensed Content Issue 1

Licensed Content Pages 11

Start Page 1

End Page 11

Type of Use reuse in a thesis/dissertation

Intended publisher of other

new work

Portion figures/tables/illustrations

Number of 2

figures/tables/illustratio

ns

Format both print and electronic

Are you the author of No 
this Elsevier article?

Will you be translating? No

Order reference number

Original figure numbers Figures 1, 3a, 3b, \& 3d

Title of your

Social dynamics, network structure, and information

thesis/dissertation diffusion in fish shoals

Expected completion

Apr 2017

date

Estimated size (number 270

of pages)

Elsevier VAT number GB 494627212

Requestor Location Matthew J Hasenjager

1407 South 1st Street

Apartment 3

LOUISVILLE, KY 40208

United States

Attn: Matthew J Hasenjager

Publisher Tax ID 98-0397604

Total $\quad 0.00$ USD 
Figure 1.6

\section{ELSEVIER LICENSE TERMS AND CONDITIONS}

Apr 10, 2017

This Agreement between Matthew J Hasenjager ("You") and Elsevier ("Elsevier") consists of your license details and the terms and conditions provided by Elsevier and Copyright Clearance Center.

License Number

4085380019623

License date

Apr 10, 2017

Licensed Content

Elsevier

Publisher

Licensed Content

Publication

Simulation Modelling Practice and Theory

Licensed Content Title Modeling group structures in pedestrian crowd simulation

Licensed Content Author Fasheng Qiu, Xiaolin $\mathrm{Hu}$

Licensed Content Date February 2010

Licensed Content Volume 18

Licensed Content Issue 2

Licensed Content Pages 16

Start Page 190

End Page 205

Type of Use reuse in a thesis/dissertation

Intended publisher of new other

work

Portion figures/tables/illustrations

Number of 1

figures/tables/illustrations

Format both print and electronic

Are you the author of this No

Elsevier article?

Will you be translating? No 
Order reference number

Original figure numbers Figure 5

Title of your Social dynamics, network structure, and information thesis/dissertation diffusion in fish shoals

Expected completion date Apr 2017

Estimated size (number of 270

pages)

Elsevier VAT number GB 494627212

Requestor Location Matthew J Hasenjager

1407 South 1st Street

Apartment 3

LOUISVILLE, KY 40208

United States

Attn: Matthew J Hasenjager

Publisher Tax ID 98-0397604

Total $\quad 0.00$ USD 
Figure 1.7

\section{SPRINGER LICENSE TERMS AND CONDITIONS}

Apr 11, 2017

This Agreement between Matthew J Hasenjager ("You") and Springer ("Springer") consists of your license details and the terms and conditions provided by Springer and Copyright Clearance Center.

License Number $\quad 4085941376145$

License date

Licensed Content Springer

Publisher

Licensed Content Behavioral Ecology and Sociobiology

Publication

Licensed Content Title Leading from the front? Social networks in navigating groups

Licensed Content Author Nikolai W. F. Bode

Licensed Content Date Jan 1, 2012

Licensed Content Volume 66

Licensed Content Issue 6

Type of Use Thesis/Dissertation

Portion Figures/tables/illustrations

Number of 2

figures/tables/illustrations

Author of this Springer No

article

Order reference number

Original figure numbers Figures 1a, 2a-c

Title of your thesis / Social dynamics, network structure, and information diffusion dissertation in fish shoals

Expected completion date Apr 2017

Estimated size(pages) $\quad 270$

Requestor Location Matthew J Hasenjager

1407 South 1st Street 
Apartment 3

LOUIS VILLE, KY 40208

United States

Attn: Matthew J Hasenjager

Billing Type

Billing Address
Invoice

Matthew J Hasenjager

1407 South 1st Street

Apartment 3

LOUISVILLE, KY 40208

United States

Attn: Matthew J Hasenjager

0.00 USD 
Figure 1.8

\section{JOHN WILEY AND SONS LICENSE TERMS AND CONDITIONS}

Apr 10, 2017

This Agreement between Matthew J Hasenjager ("You") and John Wiley and Sons ("John Wiley and Sons") consists of your license details and the terms and conditions provided by John Wiley and Sons and Copyright Clearance Center.

License Number $\quad 4085380361880$

License date

Apr 10, 2017

Licensed Content

John Wiley and Sons

Publisher

Licensed Content

American Journal of Primatology

Publication

Licensed Content Title

Males follow females during fissioning of a group of northern muriquis

Licensed Content

Marcos Tokuda, Jean P. Boubli, Ítalo Mourthé, Patrícia

Author Izar, Carla B. Possamai, Karen B. Strier

Licensed Content Date Dec 3, 2013

Licensed Content Pages 10

Type of use

Dissertation/Thesis

Requestor type

University/Academic

Format

Print and electronic

Portion

Figure/table

Number of

1

figures/tables

Original Wiley

Figure 1

figure/table number(s)

Will you be translating? No

Title of your thesis / Social dynamics, network structure, and information dissertation diffusion in fish shoals

Expected completion Apr 2017

date 


$\begin{array}{ll}\begin{array}{l}\text { Expected size (number } \\ \text { of pages) }\end{array} & 270 \\ \text { Requestor Location } & \begin{array}{l}\text { Matthew J Hasenjager } \\ 1407 \text { South 1st Street } \\ \text { Apartment 3 }\end{array} \\ & \begin{array}{l}\text { LOUISVILLE, KY 40208 } \\ \text { United States }\end{array} \\ & \begin{array}{l}\text { Attn: Matthew J Hasenjager } \\ \text { EU826007151 }\end{array} \\ & \text { Invoice } \\ \text { Publisher Tax ID } & \text { Matthew J Hasenjager } \\ \text { Billing Type } & \text { 1407 South 1st Street } \\ \text { Billing Address } & \text { Apartment 3 } \\ & \text { LOUISVILLE, KY 40208 } \\ & \text { United States } \\ & \text { Attn: Matthew J Hasenjager } \\ & \text { 0.00 USD }\end{array}$


Figure 1.9

\section{JOHN WILEY AND SONS LICENSE TERMS AND CONDITIONS}

Apr 10, 2017

This Agreement between Matthew J Hasenjager ("You") and John Wiley and Sons ("John Wiley and Sons") consists of your license details and the terms and conditions provided by John Wiley and Sons and Copyright Clearance Center.

License Number $\quad 4085380670875$

License date

Apr 10, 2017

Licensed Content

John Wiley and Sons

Publisher

Licensed Content

Ecology Letters

Publication

Licensed Content Title Individual personalities predict social behaviour in wild networks of great tits (Parus major)

Licensed Content $\quad$ L. M. Aplin, D. R. Farine, J. Morand-Ferron, E. F. Cole, Author A. Cockburn, B. C. Sheldon

Licensed Content Date Sep 17, 2013

Licensed Content Pages 8

Type of use

Dissertation/Thesis

Requestor type

University/Academic

Format

Print and electronic

Portion

Figure/table

Number of

$$
1
$$

figures/tables

Original Wiley

Figure 1

figure/table number(s)

Will you be translating? No

Title of your thesis / Social dynamics, network structure, and information dissertation diffusion in fish shoals

Expected completion Apr 2017

date 


$\begin{array}{ll}\begin{array}{l}\text { Expected size (number } \\ \text { of pages) }\end{array} & 270 \\ \text { Requestor Location } & \begin{array}{l}\text { Matthew J Hasenjager } \\ 1407 \text { South 1st Street } \\ \text { Apartment 3 }\end{array} \\ & \begin{array}{l}\text { LOUISVILLE, KY 40208 } \\ \text { United States }\end{array} \\ & \begin{array}{l}\text { Attn: Matthew J Hasenjager } \\ \text { EU826007151 }\end{array} \\ & \text { Invoice } \\ \text { Publisher Tax ID } & \text { Matthew J Hasenjager } \\ \text { Billing Type } & \text { 1407 South 1st Street } \\ \text { Billing Address } & \text { Apartment 3 } \\ & \text { LOUISVILLE, KY 40208 } \\ & \text { United States } \\ & \text { Attn: Matthew J Hasenjager } \\ & \text { 0.00 USD }\end{array}$


Figure 1.10

\section{NATURE PUBLISHING GROUP LICENSE TERMS AND CONDITIONS}

Apr 10, 2017

This Agreement between Matthew J Hasenjager ("You") and Nature Publishing Group ("Nature Publishing Group") consists of your license details and the terms and conditions provided by Nature Publishing Group and Copyright Clearance Center.

License Number

4085380905634

License date

Apr 10, 2017

Licensed Content

Nature Publishing Group

Publisher

Licensed Content Nature

Publication

Licensed Content Title A simple rule for the evolution of cooperation on graphs and social networks

Licensed Content Author Hisashi Ohtsuki, Christoph Hauert, Erez Lieberman and Martin A. Nowak

Licensed Content Date May 25, 2006

Licensed Content Volume 441

Licensed Content Issue 7092

Type of Use reuse in a dissertation / thesis

Requestor type academic/educational

Format print and electronic

Portion figures/tables/illustrations

Number of 1

figures/tables/illustrations

High-res required no

Figures $\quad$ Figure 1

Author of this NPG article no

Your reference number

Title of your thesis / dissertation

Social dynamics, network structure, and information diffusion in fish shoals 
Expected completion date Apr 2017

Estimated size (number of 270

pages)

Requestor Location

Matthew J Hasenjager

1407 South 1st Street

Apartment 3

LOUISVILLE, KY 40208

United States

Attn: Matthew J Hasenjager

Billing Type Invoice

Billing Address

Matthew J Hasenjager

1407 South 1st Street

Apartment 3

LOUISVILLE, KY 40208

United States

Attn: Matthew J Hasenjager

Total

0.00 USD 
Figure 1.11

\section{ELSEVIER LICENSE TERMS AND CONDITIONS}

Apr 10, 2017

This Agreement between Matthew J Hasenjager ("You") and Elsevier ("Elsevier") consists of your license details and the terms and conditions provided by Elsevier and Copyright Clearance Center.

License Number

License date

Licensed Content Publisher

Licensed Content Publication

Licensed Content Title

Licensed Content Author

Licensed Content Date

Licensed Content Volume

Licensed Content Issue

Licensed Content Pages

Start Page

End Page

Type of Use

Intended publisher of new work

Portion

Number of figures/tables/illustrations

Format

Are you the author of this Elsevier article?

Will you be translating?

Order reference number

Original figure numbers

Title of your thesis/dissertation
4085381076861

Apr 10, 2017

Elsevier

Animal Behaviour

A social network perspective on measurements of dominance hierarchies

Daizaburo Shizuka, David B. McDonald April 2012

83

4

10

925

934

reuse in a thesis/dissertation

other

figures/tables/illustrations

1

both print and electronic

No

No

Figure 1

Social dynamics, network structure, and 
Expected completion date

Estimated size (number of pages)

Elsevier VAT number

Requestor Location

Publisher Tax ID

Total information diffusion in fish shoals

Apr 2017

270

GB 494627212

Matthew J Hasenjager

1407 South 1st Street

Apartment 3

LOUISVILLE, KY 40208

United States

Attn: Matthew J Hasenjager

98-0397604

0.00 USD 
Figure 1.12

\section{JOHN WILEY AND SONS LICENSE TERMS AND CONDITIONS}

Apr 10, 2017

This Agreement between Matthew J Hasenjager ("You") and John Wiley and Sons ("John Wiley and Sons") consists of your license details and the terms and conditions provided by John Wiley and Sons and Copyright Clearance Center.

License Number $\quad 4085381288278$

License date

Apr 10, 2017

Licensed Content

John Wiley and Sons

Publisher

Licensed Content

Methods in Ecology \& Evolution

Publication

Licensed Content Title Temporal dynamics and network analysis

Licensed Content Benjamin Blonder, Tina W. Wey, Anna Dornhaus,

Author

Richard James, Andrew Sih

Licensed Content Date Aug 1, 2012

Licensed Content Pages 15

Type of use

Dissertation/Thesis

Requestor type

University/Academic

Format

Print and electronic

Portion

Figure/table

Number of

1

figures/tables

Original Wiley Box 2a,b

figure/table number(s)

Will you be translating? No

Title of your thesis / Social dynamics, network structure, and information dissertation diffusion in fish shoals

Expected completion Apr 2017

date

Expected size (number 270 


$\begin{array}{ll}\text { of pages) } & \\ \text { Requestor Location } & \begin{array}{l}\text { Matthew J Hasenjager } \\ \text { 1407 South 1st Street } \\ \text { Apartment 3 }\end{array} \\ & \begin{array}{l}\text { LOUISVILLE, KY 40208 } \\ \text { United States } \\ \text { Attn: Matthew J Hasenjager }\end{array} \\ & \text { EU826007151 } \\ \text { Publisher Tax ID } & \text { Invoice } \\ \text { Billing Type } & \text { Matthew J Hasenjager } \\ \text { Billing Address } & \text { 1407 South 1st Street } \\ & \text { Apartment 3 } \\ & \text { LOUISVILLE, KY 40208 } \\ & \text { United States } \\ & \text { Attn: Matthew J Hasenjager } \\ & \text { 0.00 USD }\end{array}$




\section{Copyright permission for Chapter II}

\section{OXFORD UNIVERSITY PRESS LICENSE TERMS AND CONDITIONS}

Apr 10, 2017

This Agreement between Matthew J Hasenjager ("You") and Oxford University Press ("Oxford University Press") consists of your license details and the terms and conditions provided by Oxford University Press and Copyright Clearance Center.

License Number

4085351210501

License date

Licensed content

publisher

Licensed content

publication

Licensed content title

Oxford University Press

Behavioral Ecology

Familiarity affects network structure and information flow in guppy (Poecilia reticulata) shoals

Licensed content author Hasenjager, Matthew J.; Dugatkin, Lee Alan

Licensed content date 2016-10-06

Type of Use Thesis/Dissertation

Institution name

Title of your work

Social dynamics, network structure, and information diffusion in fish shoals

Publisher of your work n/a

Expected publication date Apr 2017

Permissions cost $\quad 0.00$ USD

Value added tax $\quad 0.00$ USD

Total $\quad 0.00$ USD

Requestor Location Matthew J Hasenjager

1407 South 1st Street

Apartment 3

LOUISVILLE, KY 40208

United States

Attn: Matthew J Hasenjager 


\begin{tabular}{|c|c|}
\hline Publisher Tax ID & GB125506730 \\
\hline Billing Type & Invoice \\
\hline Billing Address & $\begin{array}{l}\text { Matthew J Hasenjager } \\
1407 \text { South 1st Street } \\
\text { Apartment } 3\end{array}$ \\
\hline & $\begin{array}{l}\text { LOUISVILLE, KY } 40208 \\
\text { United States } \\
\text { Attn: Matthew J Hasenjager }\end{array}$ \\
\hline Total & 0.00 USD \\
\hline
\end{tabular}




\section{CURRICULUM VITAE}

Matthew J. Hasenjager

(248) 214-7320 | hasenjag27@gmail.com

\section{Education}

Doctor of Philosophy - Biology

Division of Evolution, Ecology, and Behavioral Biology

University of Louisville, Louisville, Kentucky, USA

Received May 2017

Master of Science - Zoo and Aquarium Management

Michigan State University, East Lansing, Michigan, USA

Received August 2011

Bachelor of Science - Zoology

Concentrations

Animal Behavior/Neurobiology

Zoo and Aquarium Science

Michigan State University, East Lansing, Michigan, USA

Received May 2009 with High Honor

\section{Publications}

Hasenjager, M. J., \& Dugatkin, L. A. (2017). Familiarity affects network structure and information flow in guppy (Poecilia reticulata) shoals. Behavioral Ecology, 28, 233-242. doi:10.1093/beheco/arw152.

Hasenjager, M. J. (2016). Cooperation among fishes. In: T. K. Shackelford, \& V. A. Weekes-Shackelford, (Eds.), Encyclopedia of Evolutionary Psychological Science. New York, NY: Springer Science + Business Media. doi: 10.1007/978-3-319-16999-6_1231-1.

Hasenjager, M. J., \& Dugatkin, L. A. (2015). Social network analysis in behavioral ecology. Advances in the Study of Behavior, 47, 39-114. doi: 10.1016/bs.asb.2015.02.003. 
Dugatkin, L. A., \& Hasenjager, M. (2015). The networked animal. Scientific American, 312, 50-55. doi: 10.1038/scientificamerican0615-50.

Hasenjager, M. J., \& Bergl, R. (2015). Environmental conditions associated with repetitive behavior in a group of African elephants. Zoo Biology, 34, 201210. doi: 10.1002/zoo.21211.

\section{Presentations/Interviews}

Hasenjager, M. J., \& Dugatkin, L. A. (2017). Group behavioral composition influences social network structure and information flow in guppy shoals. The Center for the Integrative Study of Animal Behavior, Indiana University. Bloomington, IN. Oral presentation.

Hasenjager, M. J., \& Dugatkin, L. A. (2017). The effects of group behavioral composition on social network structure and information flow in guppy shoals. Workshop: Emergent Properties of Individual Behavior. Ecological Research and Education Center, University of Kentucky. Lexington, KY. Oral presentation.

Hasenjager, M. J., \& Dugatkin, L. A. (2016). The effects of behavioral composition on network dynamics and information flow in Trinidadian guppy shoals. Animal Behavior Society Annual Conference. Columbia, MO. Oral presentation.

Hasenjager, M. J., \& Dugatkin, L. A. (2016). Familiarity affects network structure and information flow in guppy (Poecilia reticulata) shoals. The Center for the Integrative Study of Animal Behavior, Indiana University. Bloomington, IN. Oral presentation.

Sirius XM Science Radio. Interviewed by Dr. Anthony Atala on animal social networks. September $26^{\text {th }}, 2015$.

Hasenjager, M. J., \& Dugatkin, L. A. (2015). The effects of familiarity in shoaling fish: social dynamics, network structure, and social learning. Animal Behavior Society Annual Conference. Anchorage, AK. Oral presentation.

Hasenjager, M. J., \& Dugatkin, L. A. (2015). The effects of familiarity on information diffusion through guppy social networks. The Center for the Integrative Study of Animal Behavior, Indiana University. Bloomington, IN. Poster presentation.

Hasenjager, M. J. (2015). Animal social networks: Exploring the interconnectedness of animal societies. TEDxUofL 2015 speaker. 
Hasenjager, M. J., \& Dugatkin, L. A. (2014). The effects of familiarity on information diffusion through social networks. International Society of Behavioral Ecology Biannual Conference. New York City, NY. Poster presentation.

\section{Grants}

2017

Biology Graduate Student Association Travel Grant (\$100)

2016

Kentucky Science and Engineering Foundation $(\$ 28,424)$

Research Development and Excellence Program

Proposal Title: Social networks: A behavioral ecology approach.

While not listed on the grant as a co-principal investigator, I contributed substantially to writing the proposal and developing the ideas contained therein.

Biology Graduate Student Association Travel Grant (\$150)

Graduate Student Council Research Grant (\$300)

2015

Biology Graduate Student Association Research Grant (\$200)

Graduate Student Council Research Grant (\$280)

2014

Animal Behavior Society Student Research Grant $(\$ 1,100)$

Biology Graduate Student Association Travel Grant (\$150)

Biology Graduate Student Association Research Grant (\$55)

2013

Fisheries Society of the British Isles Small Research Grant (£502.89)

Graduate Student Council Research Grant (\$300)

2012

University of Louisville Research Initiation Grant $(\$ 5,000)$

\section{Awards}

Best Presentation Award. (2016). Department of Biology, University of Louisville

Stuart E. Neff Award. (2015). Department of Biology, University of Louisville

Best Publication Award. (2015). Department of Biology, University of Louisville

Graduate Research Fellowship. (2011). University of Louisville 


\section{Research Experience}

Doctoral Dissertation Research (August 2011-May 2017)

Department of Biology, University of Louisville

Dissertation: Social dynamics, network structure, and information

diffusion in fish shoals.

Elephant Behavior Research Intern - North Carolina Zoo (May-August 2011)

Master's Thesis Research (August 2009-May 2011)

Department of Zoology, Michigan State University

Thesis: Effects of increased feeding frequency on the activity budgets of captive North American river otters (Lontra canadensis).

Enrichment and Research Intern - Toledo Zoo (May-August 2009)

\section{Teaching Experience}

Graduate Teaching Assistant - University of Louisville Introduction to Biological Systems Laboratory (August 2015-June 2016)

Principles of Biology Laboratory (July 2014)

Genetics Laboratory (August 2012-May 2014)

Graduate Teaching Assistant - Michigan State University

Organisms and Populations Laboratory (August 2010-May 2011)

Applications in Biological Science Laboratory (August-December 2009)

Enrichment and Research Intern - The Toledo Zoo (May-August 2009)

Provided public talks to zoo visitors regarding wildlife conservation, as well as communicated key concepts to elementary and middle school students about enrichment and animal training at the zoo.

\section{Service, Outreach, and Related Professional Experience}

Biology Graduate Student Association - University of Louisville

Treasurer (April 2015-April 2017)

Vice President (April 2015-April 2016)

Graduate Committee Representative (September 2013-May 2014)

Grant Writing Academy - School of Interdisciplinary and Graduate Studies, University of Louisville (September-December 2015).

Graduate Network of Arts and Sciences - University of Louisville

Representative for the Biology Department (April 2015-April 2016) 
Have served as a reviewer for:

Animal Behaviour

Behavioral Ecology and Sociobiology

Participated in the $2^{\text {nd }}$ Social Learning Strategies Tournament (February 2012)

Organized by Dr. Luke Rendell

http://lalandlab.st-andrews.ac.uk/tournaments/

Strategy name: parasiticLearner

ORNIS Project - Michigan State University

Georeferenced museum bird specimen locality data (November 2008April 2009).

Animal Welfare Judging Competition - Michigan State University (November 2008).

Mammal Department Intern - Detroit Zoological Society (May-August 2008)

\section{Professional Society Memberships}

Animal Behavior Society

International Society for Behavioral Ecology

Kentucky Academy of Science 